Nevada

Environmental

Restoration

Project

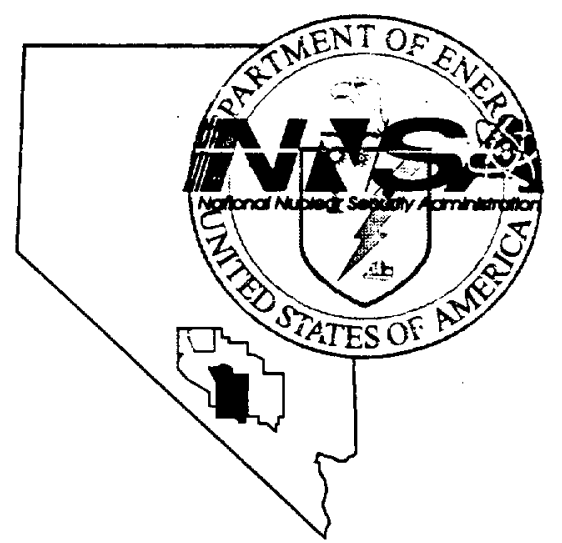

Closure Report for Housekeeping Category Corrective Action Unit 387: Spill Sites and Releases, Nevada Test Site, Nevada

Controlled Copy No::

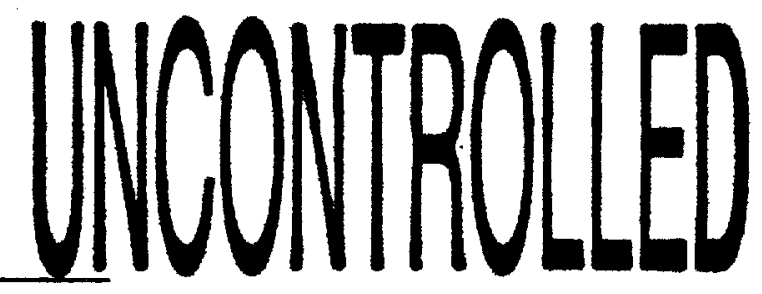

Revision: 0

October 2001

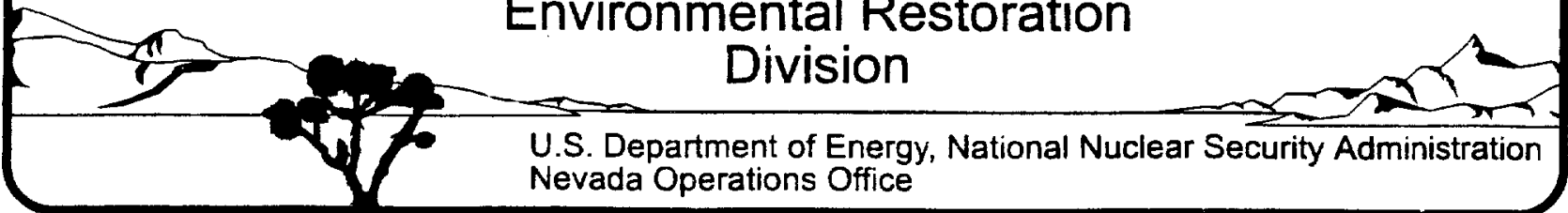




\section{DISCLAIMER STATEMENT}

Reference herein to any specific commercial product, process. or service by trade name. trademark, manufacturer, or otherwise. does not necessarily constitute or imply its endorsement, recommendation, or favoring by the U.S. Government or any agency thereof or its contractors or subcontractors.

\section{AVAILABILITY STATEMENT}

Available for sale to the public from-

U.S. Department of Commerce

National Technical Information Service

5285 Port Royal Road

Springfield, VA 22161-0002

Telephone: 800.553 .6847

Fax: 703.605.6900

E-mail orders@ntis.fedworld.gov

Online ordering: http://www.ntis.gov/ordering.htm

Available electronically at http://www.doe.gov/bridge

Available for a processing fee to U.S. Department of Energy and its contractors, in paper, fromU.S. Department of Energy

Office of Scientific and Technical Information

P.O. Box 62

Oak Ridge, TN 37831-0062

Telephone: 865.576 .8401

Fax: 865.576.5728

E-mail: reports $\underline{a}$ adonis.osti.gov 


\title{
CLOSURE REPORT FOR HOUSEKEEPING CATEGORY CORRECTIVE ACTION UNIT 387: SPILL SITES AND RELEASES, NEVADA TEST SITE, NEVADA
}

\author{
Prepared for: \\ U.S. Department of Energy \\ National Nuclear Security Administration \\ Nevada Operations Office \\ Work Performed Under Contract No. DE-AC08-96-NV11718
}

Controlled Copy No:

Revision: 0

October 2001 


\section{CLOSURE REPORT FOR HOUSEKEEPING CATEGORY CORRECTIVE ACTION UNIT 387: SPILL SITES AND RELEASES, NEVADA TEST SITE, NEVADA}

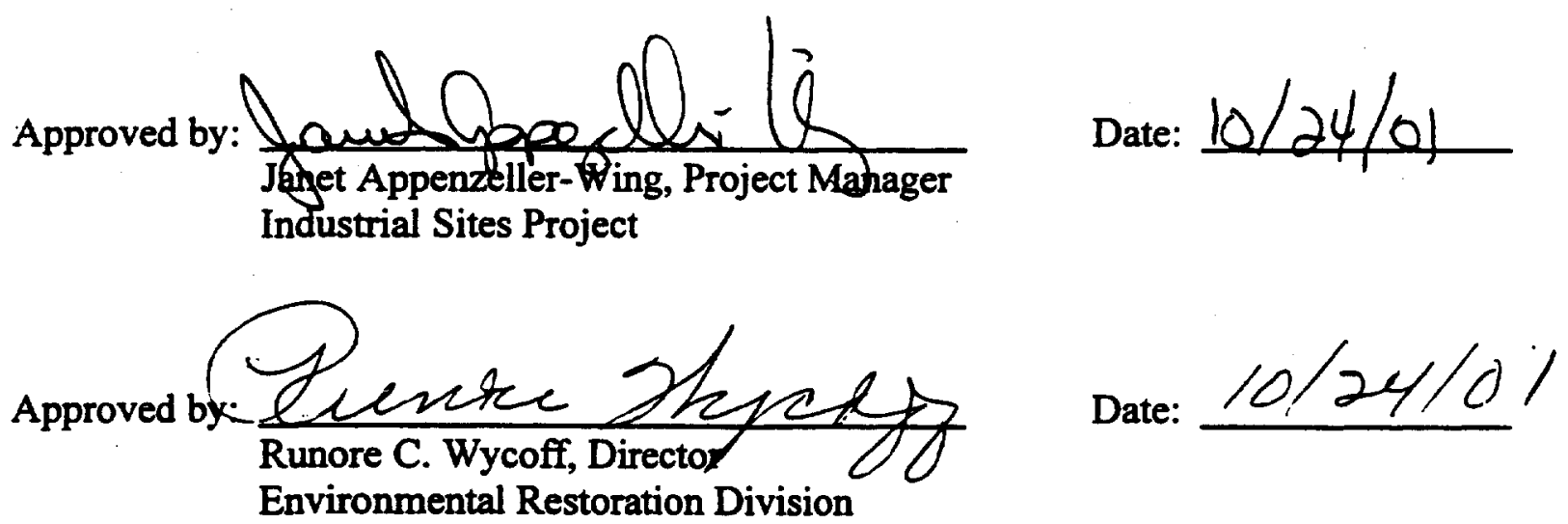


THIS PAGE INTENTIONALLY LEFT BLANK 


\section{TABLE OF CONTENTS}

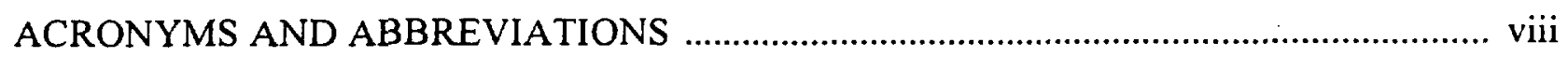

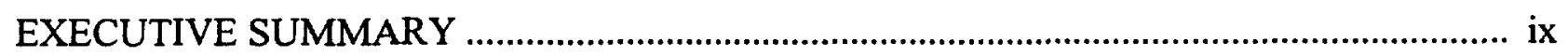

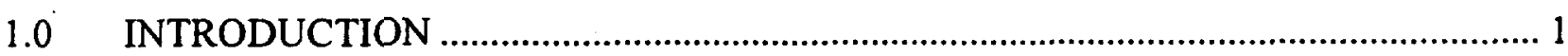

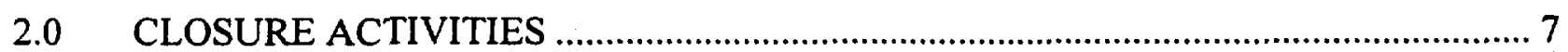

$2.1 \quad$ Description Of Closure Activities ................................................................... 7

2.1.1 Preplanning and Site Preparation ............................................................ 7

$2.2 \quad$ Waste Characterization Activities ........................................................................ 7

2.2.1 CAS 02-99-02 Waste Characterization …………………….................... 7

2.2.2 CAS 02-99-03 Waste Characterization ................................................ 10

2.2.3 CAS 02-99-04 Waste Characterization ................................................. 10

2.2.4 CAS 02-99-07 Waste Characterization ................................................. 10

2.2.5 CAS 02-99-09 Waste Characterization .................................................. 10

2.2.6 CAS 02-99-11 Waste Characterization ................................................. 10

2.2.7 CAS 02-44-01 Waste Characterization ................................................. 10

2.2.8 CAS 03-22-22 Waste Characterization ................................................ 11

2.2.9 CAS 03-22-35 Waste Characterization ................................................ 11

2.2.10 CAS 03-99-01 Waste Characterization ...................................................... 11

2.2.11 CAS 03-99-02 Waste Characterization ..................................................... 11

2.2.12 CAS 03-99-03 Waste Characterization ................................................. 11

2.2.13 CAS 03-99-06 Waste Characterization ................................................ 12

2.2.14 CAS 03-99-07 Waste Characterization ................................................. 12

2.2.15 CAS 03-99-08 Waste Characterization ..................................................... 12

2.2.16 CAS 03-99-09 Waste Characterization ............................................... 12

2.2.17 CAS 03-99-17 Waste Characterization ................................................... 12

2.2.18 CAS 03-99-18 Waste Characterization ................................................. 13

2.2.19 CAS 03-44-03 Waste Characterization ............................................... 13

2.2.20 CAS 04-44-01 Waste Characterization .................................................. 13

2.2.21 CAS 04-44-03 Waste Characterization .................................................. 13

2.2.22 CAS 07-99-02 Waste Characterization ................................................ 13

2.2.23 CAS 07-99-03 Waste Characterization .................................................... 13

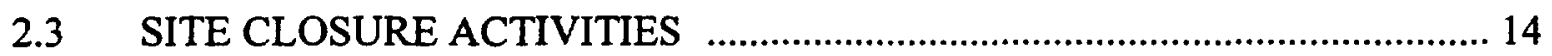

2.3.1 CAS 02-99-02: Epoxy Tar Spill …………….................................... 14

2.3.2 CAS 02-99-03: Epoxy Tar Spills .................................................... 14

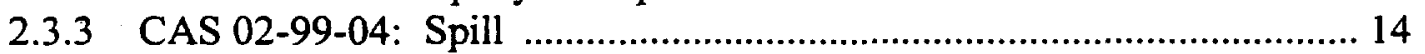

2.3.4 CAS 02-99-11: Spill ...................................................................... 14

2.3.5 CAS 03-22-22: Bucket; Spill ........................................................... 15

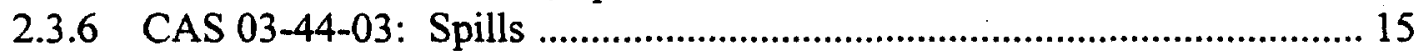

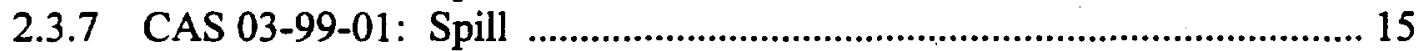

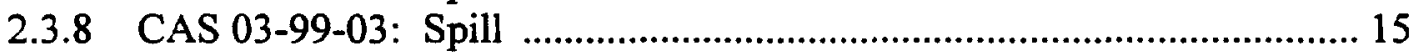

2.3.9 CAS 03-99-06: Spill ................................................................... 18

2.3.10 CAS 03-99-08: Tar Spill .............................................................. 18

2.3.11 CAS 03-99-17: Bottle Spill ................................................................. 18 


\section{TABLE OF CONTENTS (continued)}

2.3.12 CAS 03-99-18: Cement Spill ............................................................. 18

2.3.13 CAS 04-44-01: Spill ...................................................................... 18

2.3.14 CAS 04-44-03: U-4t Postshot Hole Release ........................................... 19

2.3.15 CAS 07-99-02: Discolored Area ……………..................................... 19

2.3.16 CAS 07-99-03: Compressed Bottle; Tar Spill ....................................... 19

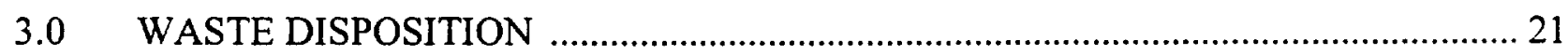

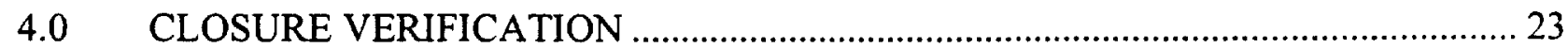

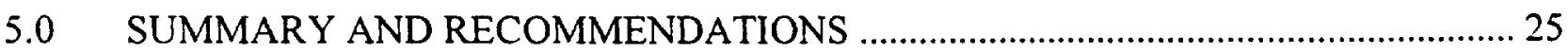

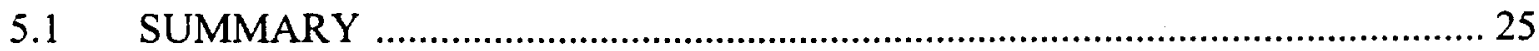

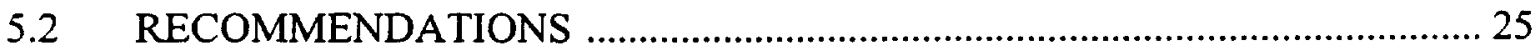

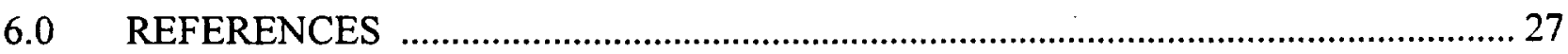

\section{APPENDICES}

APPENDIX A: VERIFICATION SAMPLE ANALYTICAL RESULTS

APPENDIX B: SECTORED HOUSEKEEPING SITE CLOSURE VERIFICATION FORMS

DISTRIBUTION LIST

\section{FIGURES}

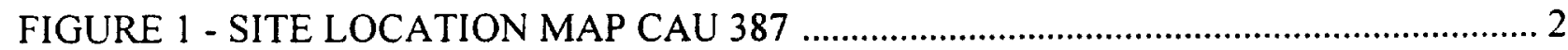

FIGURE 2 - SITE LOCATION MAP CAU 387, AREA 2 SITES …............................................ 3

FIGURE 3 - SITE LOCATION MAP, CAU 387, AREA 3 SITES …………............................. 4

FIGURE 4 - SITE LOCATION MAP, CAU 387, AREA 4 SITES ............................................... 5

FIGURE 5 - SITE LOCATION MAP, CAU 387, AREA 7 SITES …………………………..... 6 


\section{TABLE OF CONTENTS (continued)}

\section{TABLES}

TABLE 1 - SUMMARY OF ANALYTICAL RESULTS FOR WASTE CHARACTERIZATION SAMPLES

TABLE 2 - SUMMARY OF ANAL YTICAL RESULTS FOR SVOC VERIFICATION SAMPLES

TABLE 3 - SUMMARY OF ANALYTICAL RESULTS FOR TOTAL PETROLEUM HYDROCARBONS IN VERIFICATION SAMPLES 


\section{ACRONYMS AND ABBREVIATIONS}

$\mathrm{BN}$

CAS

CAU

$\mathrm{COC}$

DOE/NV

EPA

ER

FFACO

IT

LLW

m

$\mathrm{m}^{3}$

$\mathrm{mg} / \mathrm{kg}$

NAC

NDEP

NNSA/NV

NTS

PCB

RCRA

PRG

RSM

SVOC

TPH

VOC

$\mathrm{yd}^{3}$
Bechtel Nevada

Corrective Action Site(s)

Corrective Action Unit

constituent(s) of concern or contaminants of concern

U.S. Department of Energy Nevada Operations Office

U.S. Environmental Protection Agency

Environmental Restoration

Federal Facility Agreement and Consent Order

International Technology Corporation

low-level waste

meters

cubic meter(s)

milligram(s) per kilogram

Nevada Administrative Code

Nevada Division of Environmental Protection

U.S. Department of Energy, National Nuclear Security Administration Nevada Operations Office

Nevada Test Site

polychlorinated biphenyls

Resource Conservation and Recovery Act

preliminary remediation goal

RAD Safe Marker

semivolatile organic compound

total petroleum hydrocarbon

volatile organic compound

cubic yard(s) 


\section{EXECUTIVE SUMMARY}

The 27 Corrective Action Sites (CAS) that comprise Corrective Action Unit (CAU) 387 were closed by the following actions:

- CAS 02-44-01: Closed with no action required.

- CAS 02-99-01: Moved to CAU 358.

- CAS 02-99-02: Clean closed.

- CAS 02-99-03: Clean closed.

- CAS 02-99-04: Clean closed.

- CAS 02-99-07: Closed with no action required.

- CAS 02-99-09: Closed with no action required.

- CAS 02-99-11: Clean closed.

- CAS 03-22-22: Clean closed.

- $\quad$ CAS 03-22-33: Moved to CAU 358.

- CAS 03-22-35: Closed with no action required.

- $\quad$ CAS 03-44-02: Moved to CAU 536.

- CAS 03-44-03: Clean closed.

- CAS 03-99-01: Clean closed.

- CAS 03-99-02: Closed with no action required.

- CAS 03-99-03: Clean closed.

- CAS 03-99-04: Moved to CAU 358.

- CAS 03-99-06: Clean closed.

- CAS 03-99-07: Closed with no action required.

- CAS 03-99-08: Clean closed.

- CAS 03-99-09: Closed with no action required. 
- CAS 03-99-17: Clean closed.

- CAS 03-99-18: Clean closed.

- CAS 04-44-01: Clean closed.

- CAS 04-44-03: Clean closed.

- CAS 07-99-02: Clean closed.

- CAS 07-99-03: Clean closed. 


\subsection{INTRODUCTION}

This Closure Report documents the closure activities conducted for CAU 387: Spill Sites and Releases. CAU 387 is listed in Appendix III of the Federal Facility Agreement and Consent Order (FFACO) (FFACO, 1996) and consists of the following 23 CASs located in Areas 2, 3, 4, and 7 of the Nevada Test Site (NTS) (Figures 1, 2, 3, and 4):

$\begin{array}{ll}\text { - } & \text { CAS 02-99-02: Epoxy Tar Spill } \\ \text { - } & \text { CAS 02-99-03: Epoxy Tar Spills } \\ \text { - } & \text { CAS 02-99-04: Spill } \\ \text { - } & \text { CAS 02-99-07: Cement Spill } \\ \text { - } & \text { CAS 02-99-1 : }: \text { Spill } \\ \text { - } & \text { CAS 02-44-01: U-2gh Drill Site Release } \\ \text { - } & \text { CAS 03-22-22: Bucket; Spill } \\ \text { - } & \text { CAS 03-22-35: Drum; Spill } \\ \text { - } & \text { CAS 03-99-01: Spill } \\ \text { - } & \text { CAS 03-99-02: Spill } \\ \text { - } & \text { CAS 03-99-03: Spill } \\ \text { - } & \text { CAS 03-99-06: Spill } \\ \text { - } & \text { CAS 03-99-07: Spill } \\ \text { - } & \text { CAS 03-99-08: Tar Spill } \\ \text { - } & \text { CAS 03-99-17: Bottle Spill } \\ \text { - } & \text { CAS 03-99-18: Cement Spill } \\ \text { - } & \text { CAS 03-44-03: Spills } \\ \text { - } & \text { CAS 04-44-01: Spill } \\ \text { - } & \text { CAS 04-44-03: U-4tPostshot Hole Release } \\ \text { - } & \text { CAS 07-99-02: Discolored Area } \\ & \end{array}$

Closure activities were performed in two phases. Phase I activities consisted of collecting waste characterization samples of soil at appropriate sites. The results were used to determine how waste generated during closure activities would be handled and disposed (i.e., as nonhazardous sanitary or hazardous waste). Phase 2 activities consisted of closing each CAS by removing debris and/or soil, disposing of the generated waste, and verifying that each site was clean-closed by visual inspection and/or collecting soil verification samples for laboratory analysis.

Additionally, seven sites were closed with no further action after concurance with Nevada Division of Environmental Protection (NDEP). Four other sites were moved into different CAUs in Appendix III of the FFACO because the housekeeping process was not adequate to close them.

Copies of the analytical results for the site verification samples are included in Appendix A. Copies of the Sectored Housekeeping Site Closure Verification Forms for each of the 16 CAS are included in Appendix B. 


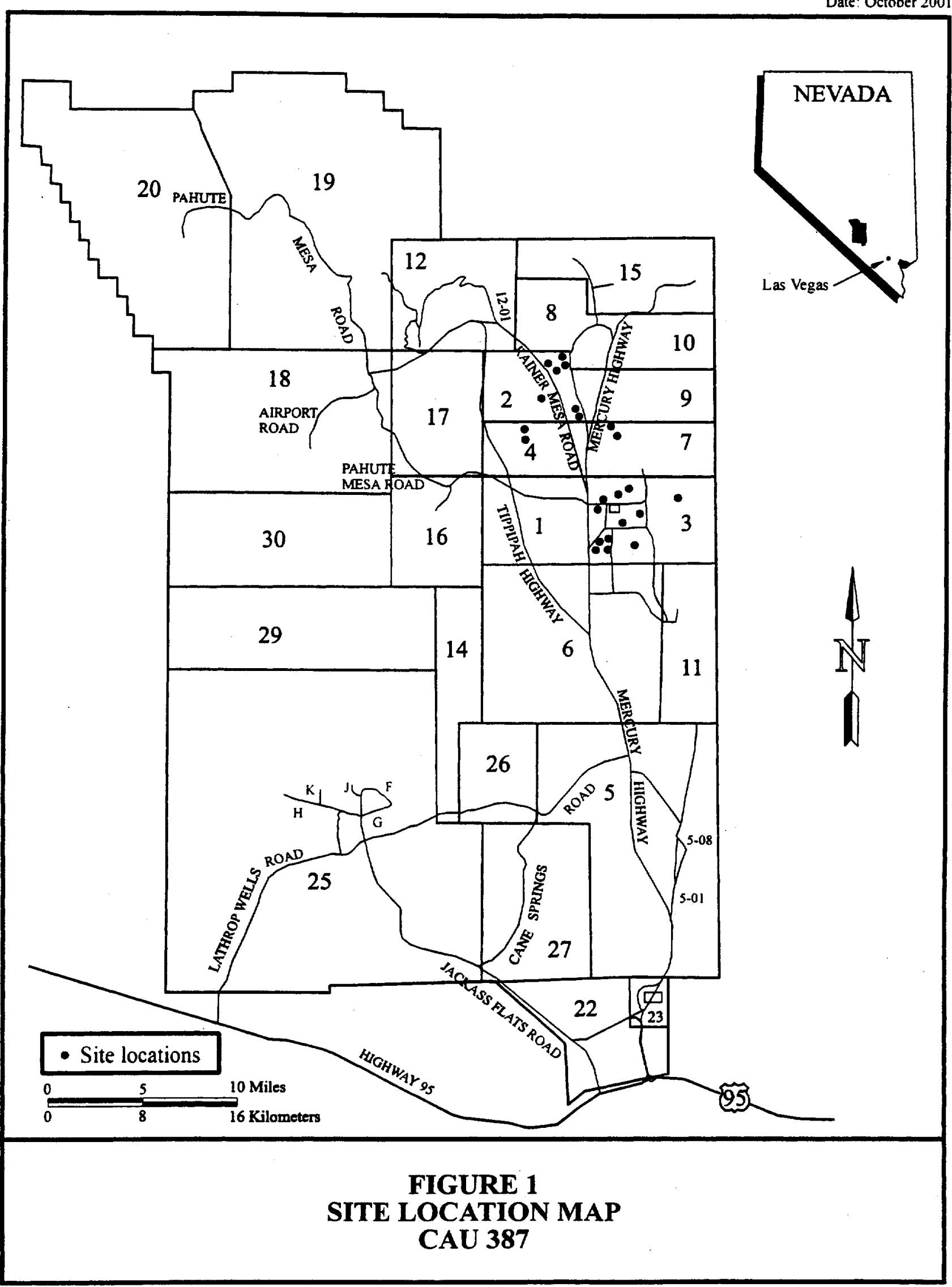




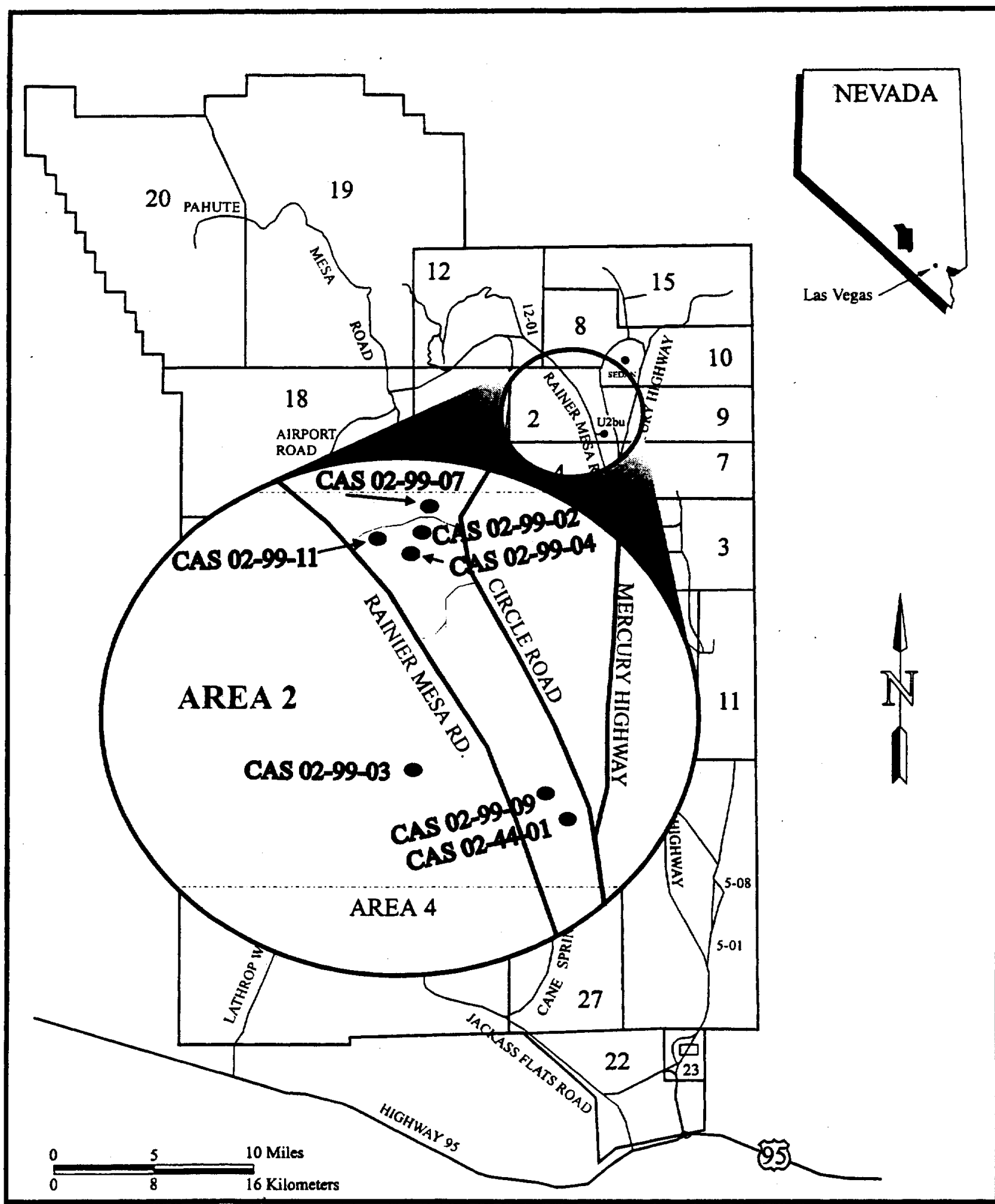

FIGURE 2

SITE LOCATION MAP

CAU 387 (AREA 2 SITES) 


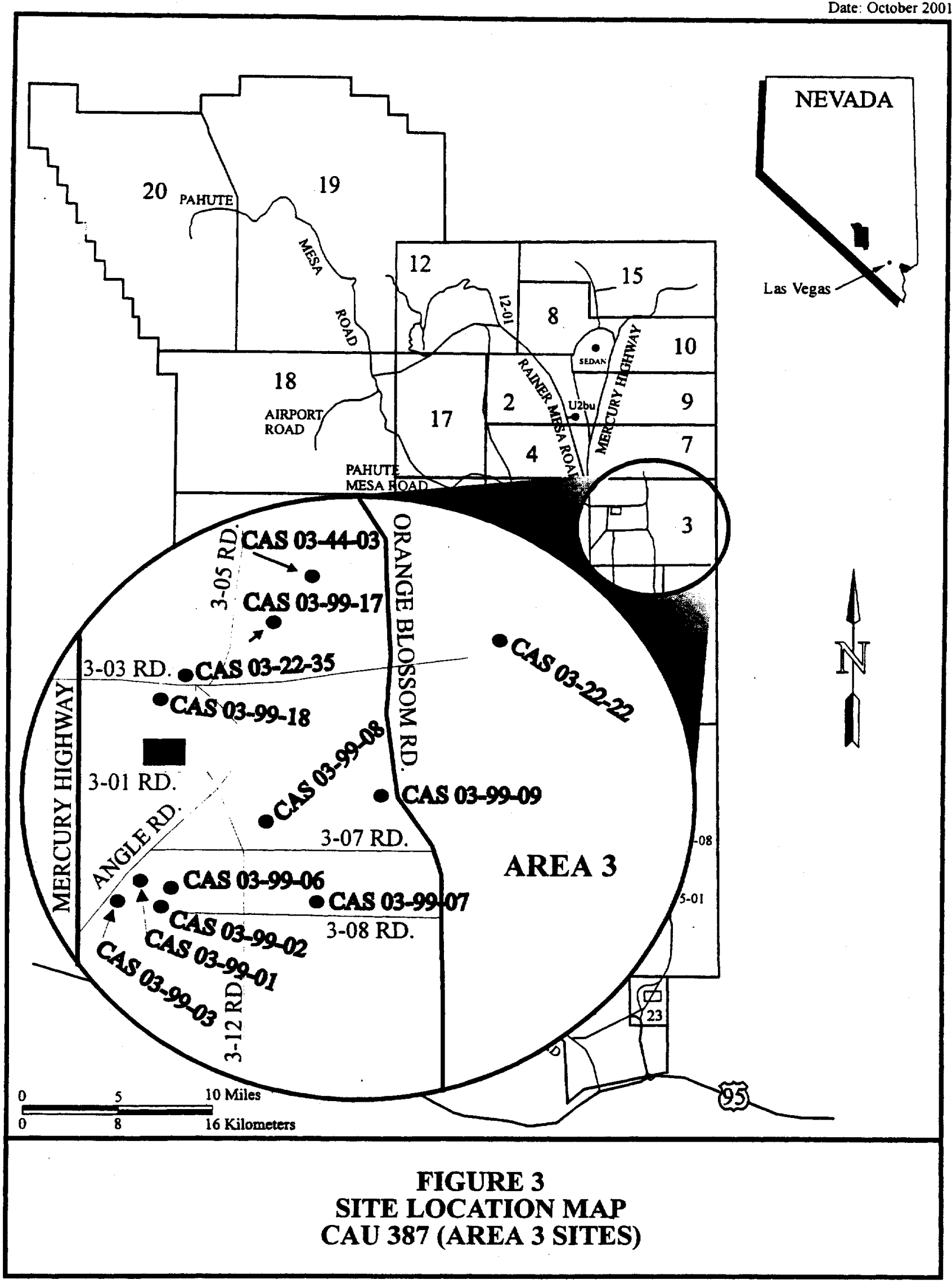




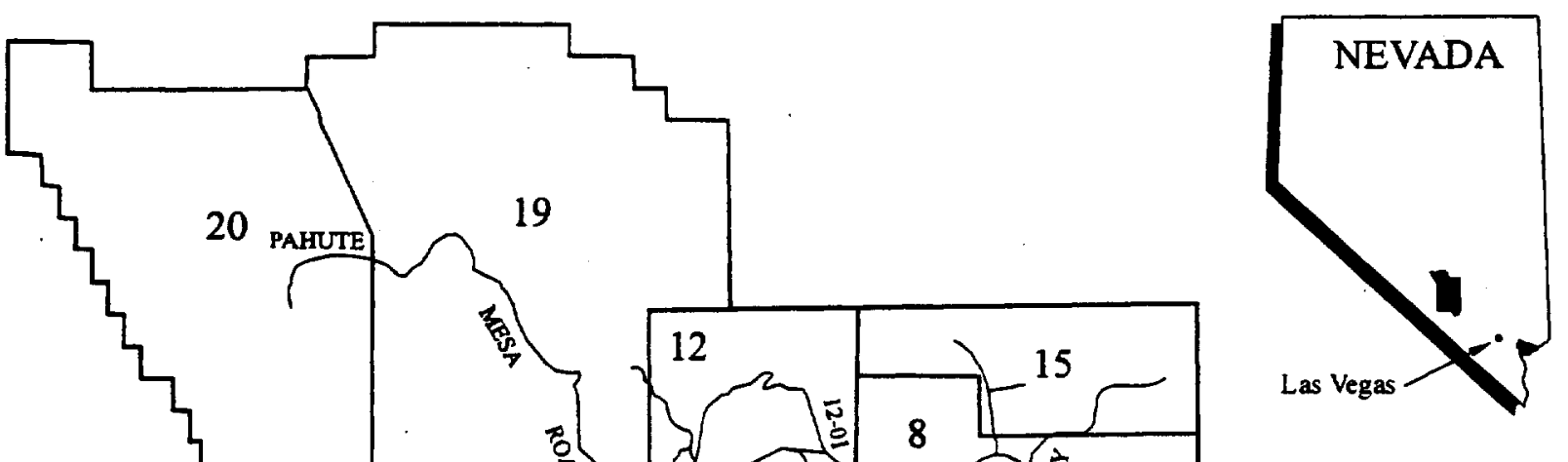




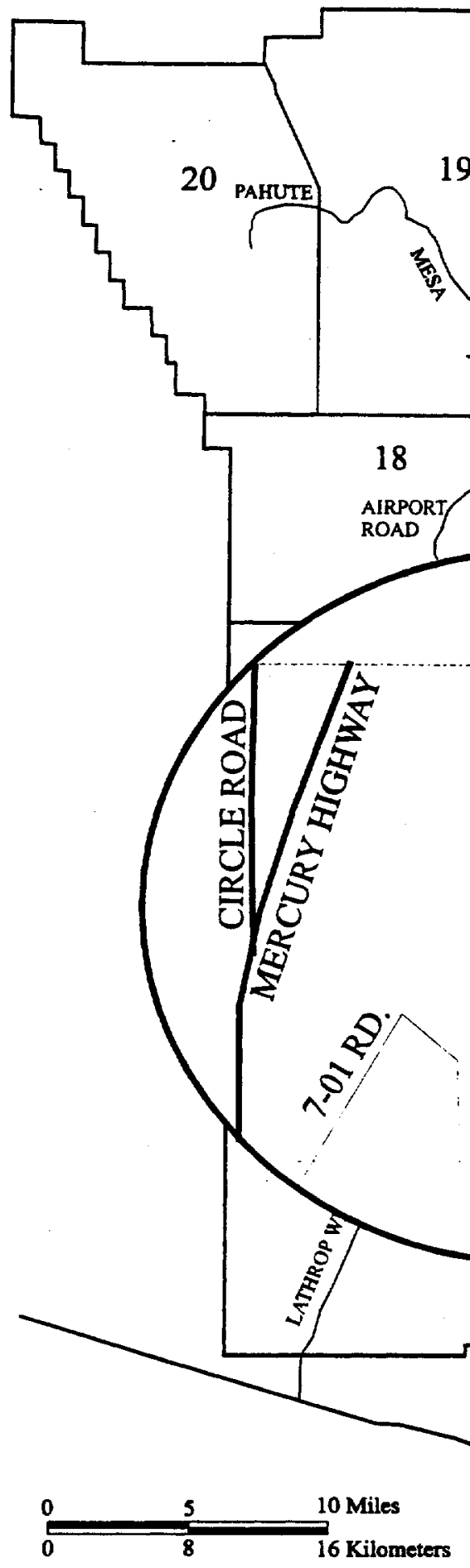

AREA 9
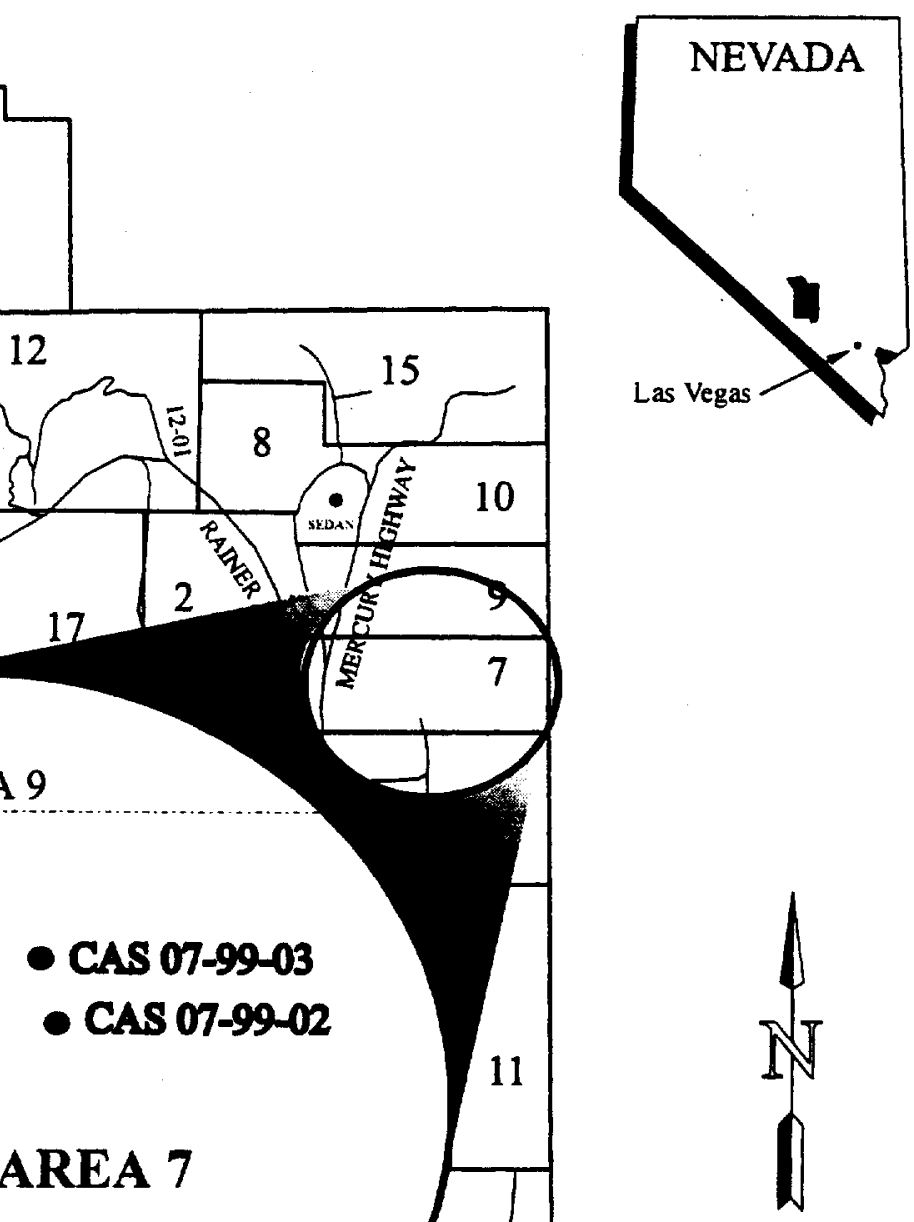


\subsection{CLOSURE ACTIVITIES}

This section details the specific corrective action activities completed during the closure of CAU 387: Spill Sites and Releases. Copies of the analytical data reports for all verification samples are included in Appendix A and copies of the Sectored Housekeeping Site Closure Verification Forms are included in Appendix B.

\subsection{Description Of Closure Activities}

\subsubsection{Preplanning and Site Preparation}

Planning documents prepared prior to the beginning of closure activities include the Sectored Clean-up Work Plan For Housekeeping Category Waste Sites (DOE/NV, 2000), Generic Field Management Plan for Housekeeping Category Waste Sites (BN, 2000), Site-Specific Health and Safety Plan (BN, 2001), Site Maintenance Work Packages, and DOE/NV Real Estate/Operation Permit (BN-0161-00). In addition, a National Environmental Policy Act checklist was prepared (NV-00-060) and a pre-activity site survey was conducted by a Bechtel Nevada (BN) biologist. The survey confirmed the absence of sensitive animal or plant species at all $23 \mathrm{CAS}$. A Readiness Review was held on July 18,2001 by BN Environmental Restoration. BN ER and Site Maintenance personnel held a pre-job field briefing on July 18, 2001, prior to the start of site-closure field activities.

\subsection{Waste Characterization Activities}

At 17 of the 23 CASs, samples of soil and/or material were collected and analyzed to characterize the waste that was expected to be generated during site-closure activities. Table 1 presents the analyses that were conducted for waste characterization samples, the results of these analyses, and the resulting waste classifications.

\subsubsection{CAS 02-99-02 Waste Characterization}

Waste generated at this site included epoxy tar/associated soil and debris, which included wood, metal and rope. On June 7, 2001, the soil samples (029902-0-1) were analyzed for total petroleum hydrocarbon (TPH), total Resource Conservation and Recovery Act (RCRA) metals, polychlorinated biphenyls (PCBs), volatile organic compounds (VOCs), semi-volatile organic compounds (SVOCs), and gamma spectroscopy. Results for total TPH were higher than the state of Nevada action level (100 milligrams per kilogram [mg/kg]) (Nevada Administrative Code [NAC, 2000]). Results for SVOCs were higher than the Region 9 preliminary remediation goals (PRGs) for industrial soils (U.S. Environmental Protection Agency [EPA, 1996a]). Results from radiological screening showed radioactivity possibly above background levels, which indicated that any waste generated might be potentially classified as low-level waste (LLW). 
TABLE 1 - SUMMARY OF ANALYTICAL RESULTS FOR WASTE CHARACTERIZATION SAMPLES

\begin{tabular}{|c|c|c|c|c|}
\hline CAS & $\begin{array}{l}\text { SAMPLE } \\
\text { TYPE }\end{array}$ & ANALYSIS & ANALYTICAL RESULTS & WASTE TYPE \\
\hline $02-99-02$ & Soil & $\begin{array}{l}\text { Total RCRA metals } \\
\text { VOCs } \\
\text { full-scan } \mathrm{TPH}^{\mathrm{e}} \text {, and } \\
\text { gamma spectroscopy } \\
\\
\text { Radiological screening }\end{array}$ & $\begin{array}{l}\text { Several SVOCs higher than action } \\
\text { levels. } \\
\text { Full-scan TPH higher than action } \\
\text { levels. } \\
\text { Radioactivity possibly above } \\
\text { background levels. }\end{array}$ & $\begin{array}{c}\text { Hydrocarbon } \\
\text { Potential LLW } \\
\text { (pending analysis) }\end{array}$ \\
\hline $02-99-03$ & Soil & $\begin{array}{l}\text { Total RCRA metals, } \\
\text { VOCs, SVOCs, PCBs, } \\
\text { full-scan TPH, and } \\
\text { gamma spectroscopy. }\end{array}$ & $\begin{array}{l}\text { Several SVOCs higher than action } \\
\text { levels. } \\
\text { Full-scan TPH higher than action } \\
\text { levels. }\end{array}$ & Hydrocarbon \\
\hline $02-99-04$ & NA & $N / A$ & N/A & $\begin{array}{c}\text { Sanitary (based on } \\
\text { process } \\
\text { knowledge) }\end{array}$ \\
\hline $02-99-07$ & Soil & $\begin{array}{l}\text { Total RCRA metals, } \\
\text { VOCs, SVOCs, full-scan } \\
\text { TPH, and gamma } \\
\text { spectroscopy. }\end{array}$ & $\begin{array}{l}\text { All analyses lower than action } \\
\text { levels. }\end{array}$ & $\begin{array}{l}\text { No further action } \\
\text { No COCs present }\end{array}$ \\
\hline $02-99-09$ & Soil & $\begin{array}{l}\text { Total RCRA metals, } \\
\text { VOCs, SVOCs, full-scan } \\
\text { TPH, and gamma } \\
\text { spectroscopy. }\end{array}$ & $\begin{array}{l}\text { All analyses lower than action } \\
\text { levels. }\end{array}$ & $\begin{array}{l}\text { No further action } \\
\text { No COCs present }\end{array}$ \\
\hline $02-99-11$ & Soil & $\begin{array}{l}\text { Total RCRA metals, } \\
\text { VOCs, SVOCs, PCBs, } \\
\text { full-scan TPH, and } \\
\text { gamma spectroscopy. }\end{array}$ & $\begin{array}{l}\text { Full-scan TPH higher than action } \\
\text { levels. }\end{array}$ & Hydrocarbon \\
\hline $02-44-01$ & $\mathrm{~N} / \mathrm{A}$ & N/A & $\mathrm{N} / \mathrm{A}$ & $\begin{array}{l}\text { No further action } \\
\text { No site was found }\end{array}$ \\
\hline $03-22-22$ & Soil & $\begin{array}{l}\text { Total RCRA metals, } \\
\text { VOCs, SVOCs, PCBs, } \\
\text { full-scan TPH, and } \\
\text { gamma spectroscopy. }\end{array}$ & $\begin{array}{l}\text { All analyses lower than action } \\
\text { levels. }\end{array}$ & Sanitary \\
\hline $03-22-35$ & $\mathrm{~N} / \mathrm{A}$ & $\mathrm{N} / \mathrm{A}$ & $\mathrm{N} / \mathrm{A}$ & $\begin{array}{l}\text { No further action } \\
\text { No drum or spill } \\
\text { found at site. }\end{array}$ \\
\hline $03-99-01$ & Soil & $\begin{array}{l}\text { Total RCRA metals, } \\
\text { VOCs, SVOCs, PCBs, } \\
\text { full-scan TPH, and } \\
\text { gamma spectroscopy. }\end{array}$ & $\begin{array}{l}\text { Several SVOCs higher than action } \\
\text { levels. } \\
\text { Full-scan TPH higher than action } \\
\text { levels. }\end{array}$ & Hydrocarbon \\
\hline $03-99-02$ & Soil & $\begin{array}{l}\text { Total RCRA metals, } \\
\text { VOCs, SVOCs, full-scan } \\
\text { TPH, and gamma } \\
\text { spectroscopy. }\end{array}$ & $\begin{array}{l}\text { All analyses lower than action } \\
\text { levels. }\end{array}$ & $\begin{array}{l}\text { No further action } \\
\text { No COCs present }\end{array}$ \\
\hline $03-99-03$ & Soil & $\begin{array}{l}\text { Total RCRA metals, } \\
\text { VOCs, SVOCs, PCBs, } \\
\text { full-scan TPH, and } \\
\text { gamma spectroscopy. }\end{array}$ & $\begin{array}{l}\text { Several SVOCs higher than action } \\
\text { levels. } \\
\text { Full-scan TPH higher than action } \\
\text { levels. }\end{array}$ & Hydrocarbon \\
\hline $03-99-06$ & Soil & $\begin{array}{l}\text { Total RCRA metals, } \\
\text { VOCs, SVOCs, PCBs, } \\
\text { full-scan TPH, and } \\
\text { gamma spectroscopy. }\end{array}$ & $\begin{array}{l}\text { Several SVOCs higher than action } \\
\text { levels. } \\
\text { Full-scan TPH higher than action } \\
\text { levels. }\end{array}$ & Hydrocarbon \\
\hline
\end{tabular}


TABLE 1 -SUMMARY OF ANALYTICAL RESULTS FOR WASTE

\section{CHARACTERIZATION SAMPLES (continued)}

\begin{tabular}{|c|c|c|c|c|}
\hline $03-99-07$ & $\mathrm{~N} / \mathrm{A}$ & N/A & $\overline{\mathrm{N} / \mathrm{A}}$ & $\begin{array}{l}\text { No further action } \\
\text { No spill found }\end{array}$ \\
\hline $03-99-08$ & Soil & $\begin{array}{l}\text { Total RCRA metals, } \\
\text { VOCs, SVOCs, PCBs, } \\
\text { full-scan TPH, and } \\
\text { gamma spectroscopy. } \\
\text { Radiological screening. }\end{array}$ & $\begin{array}{l}\text { Full-scan TPH higher than action } \\
\text { levels. } \\
\text { Radioactivity possibly above } \\
\text { background levels. }\end{array}$ & $\begin{array}{l}\text { Hydrocarbon } \\
\text { Potential LLW }\end{array}$ \\
\hline $03-99-09$ & Soil & $\begin{array}{l}\text { Total RCRA metals, } \\
\text { VOCs, SVOCs, PCBs, } \\
\text { full-scan TPH, asbestos } \\
\text { and alpha,gamma, beta } \\
\text { spectroscopy. }\end{array}$ & $\begin{array}{l}\text { All analyses lower than action } \\
\text { levels. }\end{array}$ & $\begin{array}{l}\text { No further action } \\
\text { No COCs present }\end{array}$ \\
\hline $03-99-17$ & $\begin{array}{l}\text { Soil and } \\
\text { liquid }\end{array}$ & $\begin{array}{l}\text { Total RCRA metals, } \\
\text { VOCs, SVOCs, PCBs, } \\
\text { and full-scan TPH. }\end{array}$ & $\begin{array}{l}\text { All analyses lower than action } \\
\text { levels. }\end{array}$ & Sanitary \\
\hline $03-99-18$ & $\mathrm{~N} / \mathrm{A}$ & $\mathrm{N} / \mathrm{A}$ & $\mathrm{N} / \mathrm{A}$ & $\begin{array}{c}\text { Sanitary (based on } \\
\text { process } \\
\text { knowledge) }\end{array}$ \\
\hline $03-44-03$ & Soil & $\begin{array}{l}\text { Total RCRA metals, } \\
\text { VOCs, SVOCs, PCBs, } \\
\text { TPH full scan, and gamma } \\
\text { spectroscopy. }\end{array}$ & $\begin{array}{l}\text { Full-scan TPH higher than action } \\
\text { levels. }\end{array}$ & Hydrocarbon \\
\hline $04-44-01$ & $\mathrm{~N} / \mathrm{A}$ & N/A & N/A & $\begin{array}{c}\text { Sanitary (based on } \\
\text { process } \\
\text { knowledge) }\end{array}$ \\
\hline $04-44-03$ & Soil & $\begin{array}{l}\text { Total RCRA metals, } \\
\text { VOCs, SVOCs, PCBs, } \\
\text { TPH full scan, and gamma } \\
\text { spectroscopy. }\end{array}$ & $\begin{array}{l}\text { All analyses lower than action } \\
\text { levels. }\end{array}$ & Sanitary \\
\hline $07-99-02$ & Soil & $\begin{array}{l}\text { Total RCRA metals, } \\
\text { VOCs, SVOCs, PCBs, } \\
\text { TPH full scan, and gamma } \\
\text { spectroscopy. }\end{array}$ & $\begin{array}{l}\text { All analyses lower than action } \\
\text { levels. }\end{array}$ & Sanitary \\
\hline $07-99-03$ & Soil & $\begin{array}{l}\text { Total RCRA metals, } \\
\text { VOCs, SVOCs, PCBs, } \\
\text { TPH full scan, and gamma } \\
\text { spectroscopy. }\end{array}$ & $\begin{array}{l}\text { Several SVOCs higher than action } \\
\text { levels. } \\
\text { Full-scan TPH higher than action } \\
\text { levels. }\end{array}$ & Hydrocarbon \\
\hline
\end{tabular}

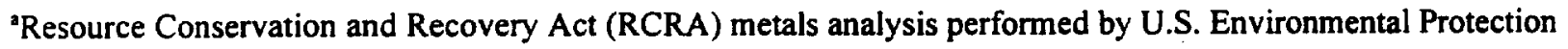
Agency (EPA) Method SW-846 6010, 7471A (EPA, 1996b).

'Volatile organic compounds (VOC) analysis performed by EPA Method SW-846 8260B (EPA, 1996b). See analytical reports in Appendix A for detection limits.

'Semivolatile organic compounds (SVOC) analysis performed by EPA Method SW-846 8270C (EPA, 1996b). See analytical reports in Appendix A for detection limits.

'Polychlorinated biphenyls analysis performed by EPA Method SW-846 8082 (EPA, 1996b). See analytical reports in Appendix A for detection limits.

'Total petroleum hydrocarbons (TPH) analysis performed by EPA Method SW-846 8015M (EPA, 1996b). See analytical reports in Appendix A for detection limits.

${ }^{i}$ Gamma spectroscopy analysis performed by EPA Method SW-846 901.1 (EPA, 1996b). See analytical reports in Appendix A for detection limits.

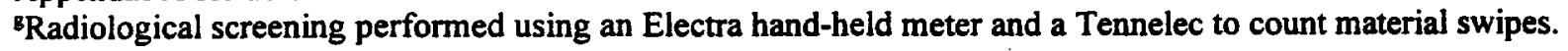




\subsubsection{CAS 02-99-03 Waste Characterization}

Waste generated at this site included epoxy tar. On June 7, 200, the soil samples (029903-0-1) were analyzed for TPH, total RCRA metals, PCBs, VOCs, SVOCs, and gamma spectroscopy. Results for TPH were higher than the state of Nevada action level (NAC, 2000). Results for SVOCs were higher than the Region 9 PRGs for industrial soils, but were not considered hazardous for disposal purposes (EPA, 1996a). Based on waste characterization results, the epoxy tar was disposed of as hydrocarbon waste.

\subsubsection{CAS 02-99-04 Waste Characterization}

Waste generated at this site included concrete/cement and debris, which included metal fence posts and wood. No waste characterization sampling was done, due to process knowledge that concrete/cement and debris are discarded construction material and do not contain constituents of concern (COCs). The waste material and debris was disposed of as sanitary waste.

\subsubsection{CAS 02-99-07 Waste Characterization}

This site was visited by NDEP and was determined that it required no further action pending analytical results confirming that no COCs were present (Liebendorfer, 2001). On September 4, 2001, the soil samples (029907-0-1) were analyzed for TPH, total RCRA metals, SVOCs, VOCs and gamma spectroscopy. Waste characterization sampling confirmed that COCs are not present. The site was left with no further action because vegetation has begun to recolonize the site and the magnitude of the disturbance to remove a spill containing no COCs.

\subsubsection{CAS 02-99-09 Waste Characterization}

This site was visited by NDEP and was determined that it required no further action pending analytical results confirming that no COCs were present (Liebendorfer, 2001). On September 4, 2001, the soil samples (029909-0-1) were analyzed for TPH, total RCRA metals, SVOCs, VOCs and gamma spectroscopy. Waste characterization sampling confirmed that COCs are not present. The site was left with no further action because vegetation has begun to recolonize the site and the magnitude of the disturbance to remove a spill containing no COCs.

\subsubsection{CAS 02-99-11 Waste Characterization}

Waste generated at this site included epoxy. On June 7, 2001, the soil samples (029911-0-1) were analyzed for TPH, total RCRA metals, PCBs, VOCs, SVOCs, and gamma spectroscopy. Results for TPH were higher than the state of Nevada action level (NAC, 2000). Based on waste characterization results, the epoxy spill was disposed of as hydrocarbon waste.

\subsubsection{CAS 02-44-01 Waste Characterization}

No release could be located for this CAS. An International Technology Corporation (IT), Las Vegas field crew attempted to locate this site, but the lack of specific site location information has made it impossible to find. BN has made two further attempts to find the site anc could not do so. It was determined by NDEP that no action was required for this CAS (Liebendorfer, 2001). 


\subsubsection{CAS 03-22-22 Waste Characterization}

Waste generated at this site included dark soil and debris, which included steel cables, drum lids, electrical box, electrical cables, bolts, and wood. On June 8, 2001, the soil samples (032222-01) were analyzed for TPH, total RCRA metals, PCBs, VOCs, SVOCs, and gamma spectroscopy. Based on waste characterization results, the waste material and debris was disposed of as sanitary waste.

\subsubsection{CAS 03-22-35 Waste Characterization}

No drum or spill could be located for this CAS. Past documentation also mentions that no drum or spill could be located from previous site visits. It was determined by NDEP that no action was required for this CAS (Liebendorfer, 2001).

\subsubsection{CAS 03-99-01 Waste Characterization}

Waste generated at this site included epoxy tar and debris, which included scrap metal, wood, and wire. On June 7, 2001, the soil samples were analyzed for TPH, total RCRA metals, PCBs, VOCs, SVOCs, and gamma spectroscopy. Results for total TPH were higher than the state of Nevada action levels (NAC, 2000). Results for SVOCs were higher than the Region 9 PRGs for industrial soils, but were not considered hazardous for disposal purposes (EPA, 1996a). Based on waste characterization results, the waste generated from closure was categorized as hydrocarbon waste.

\subsubsection{CAS 03-99-02 Waste Characterization}

This site was visited by NDEP and was determined that it required no further action pending analytical results confirming that no COCs were present (Liebendorfer, 2001). On June 11, 2001, the soil samples (039902-0-1) were analyzed for TPH and total RCRA metals. On September 4, 2001, additional samples (039902-0-1) were analyzed for SVOCs, VOCs and gamma spectroscopy. Waste characterization sampling confirmed that COCs are not present. The site was left with no further action because vegetation has begun to recolonize the site and the magnitude of the disturbance to remove a spill containing no COCs.

\subsubsection{CAS 03-99-03 Waste Characterization}

Waste generated at this site included epoxy tar and debris, which included a trash can lid and an empty paint can. On June 3, 2001, the soil samples were analyzed for TPH, total RCRA metals, PCBs, VOCs, SVOCs, and gamma spectroscopy. Results for total TPH were higher than the state of Nevada action levels (NAC, 2000). Results for SVOCs were higher than the Region 9 PRGs for industrial soils, but were not considered hazardous for disposal purposes (EPA, 1996a). Based on waste characterization results, the waste generated from closure was categorized as hydrocarbon waste. 


\subsubsection{CAS 03-99-06 Waste Characterization}

Waste generated at this site included epoxy tar. On June 11, 2001, the soil samples (039906-0-1) were analyzed for TPH, total RCRA metals, PCBs, VOCs, SVOCs, and gamma spectroscopy. Results for TPH were higher than the state of Nevada action levels (NAC, 2000). Results for SVOCs were higher than the Region 9 PRGs for industrial soils, but were not considered hazardous for disposal purposes (EPA, 1996a). Based on waste characterization results, the waste generated from closure was categorized as hydrocarbon waste.

\subsubsection{CAS 03-99-07 Waste Characterization}

No spill could be located for this CAS. Past documentation also mentions that no spill could be located from previous site visits. It was determined by NDEP that no action was required for this CAS (Liebendorfer, 2001).

\subsubsection{CAS 03-99-08 Waste Characterization}

Waste generated at this site included tar. On June 11, 2001, the soil samples (039908-0-1) were analyzed for TPH, total RCRA metals, PCBs, VOCs, SVOCs, and gamma spectroscopy. Results for TPH were higher than the state of Nevada action levels (NAC, 2000). Results for SVOCs were higher than the Region 9 PRGs for industrial soils (EPA, 1996a). Results from radiological screening showed radioactivity possibly above background levels, which indicated that any waste generated might be potentially classified as LLW.

\subsubsection{CAS 03-99-09 Waste Characterization}

This site was visited by NDEP and was determined that it required no further action pending analytical results confirming that no COCs were present (Liebendorfer, 2001). On June 8, 2001, the soil samples (039909-0-1, 2, 3 and 4) were analyzed for TPH, total RCRA metals and alpha, beta and gamma spectroscopy. On July 19, 2001, additional samples (039909-0-1 A, 2B, 3C and 4D) were analyzed for PCBs, SVOCs, VOCs and asbestos. Waste characterization sampling confirmed that COCs are not present. The site was left with no further action because vegetation has begun to recolonize the site and the magnitude of the disturbance to remove a spill containing no COCs.

\subsubsection{CAS 03-99-17 Waste Characterization}

Waste generated at this site included an amber glass bottle with unknown liquid, and metal debris. On June 8, 2001, the soil samples (039917-0-1) was collected from directly beneath the bottle and analyzed for SVOCs and VOCs. On July 19, 2001, an additional soil sample (039917-0-1A) was collected from directly beneath the bottle and analyzed for TPH, total RCRA metals, and PCBs. On August 22, 2001, the bottle and its liquid contents (082201-A) were analyzed for VOCs. The liquid inside the bottle contained no COCs. Based on waste characterization results, the waste material and debris were free to be released as sanitary waste. 


\subsubsection{CAS 03-99-18 Waste Characterization}

Waste generated at this site included cement/concrete. No waste characterization sampling was done, due to process knowledge that cement is discarded construction material and does not contain COCs. Based on waste characterization results, the waste material was free to be released as sanitary waste.

\subsubsection{CAS 03-44-03 Waste Characterization}

Waste generated at this site included stained hydrocarbon soil. On June 8, 2001, soil samples (034403-0-1 and 034403-0-2) were collected, one at each small spill and were analyzed for TPH, total RCRA metals, PCBs, VOCs, SVOCs, and gamma spectroscopy. Results for TPH were higher than the state of Nevada action level (NAC, 2000). Based on waste characterization results, the waste generated from closure was categorized as hydrocarbon waste.

\subsubsection{CAS 04-44-01 Waste Characterization}

Waste generated at this site included cement/grout material and debris, which included wire, rope, and wood. No waste characterization sampling was done, due to process knowledge that cement/grout and debris is discarded construction material and does not contain COCs. The waste material was free to be released as sanitary waste.

\subsubsection{CAS 04-44-03 Waste Characterization}

Waste generated at this site included cement/drilling material and debris, which included rope and wood. On June 18, 2001, the soil samples (044403-0-1) were analyzed for TPH, total RCRA metals, PCBs, VOCs, SVOCs, and gamma spectroscopy. Based on waste characterization results, the waste material was free to be released as sanitary waste.

\subsubsection{CAS 07-99-02 Waste Characterization}

Waste generated at this site included cement and debris, which included wood, scrap metal, wire, and rubber. On June 11, 2001, two soil samples (079902-0-1 and 079902-0-2) were analyzed. The first sample (079902-0-1) was collected near the concrete pad and analyzed for total RCRA metals; the second (079902-0-2) was collected near a pile of cement and analyzed for TPH, total RCRA metals, PCBs, VOCs, SVOCs and gamma spectroscopy. Based on waste characterization results, the waste material and debris were free to be released as sanitary waste.

\subsubsection{CAS 07-99-03 Waste Characterization}

Waste generated at this site included epoxy tar and debris, which included a wooden spool, wire, and gas cylinder. On June 13, 2001, the soil samples (079903-0-1) were analyzed for TPH, total RCRA metals, PCBs, VOCs, SVOCs, and gamma spectroscopy. Results for TPH were higher than the state of Nevada action level (NAC, 2000). Results for SVOCs were higher than the Region 9 PRGs for industrial soils, but were not considered hazardous for disposal purposes (EPA, 1996a). Based on waste characterization results, the waste and debris were categorized as 
hydrocarbon waste. The gas cylinder was free to be released as sanitary waste after being neutralized.

\subsection{SITE CLOSURE ACTIVITIES}

\subsubsection{CAS 02-99-02: Epoxy Tar Spill}

Epoxy tar and debris were removed from the site on August 17, 2001. Since they may potentially be classified LLW, the epoxy and debris were placed into B-25 containers. The containers are currently being further characterized for LLW. Closure activities were documented with photographs. Verification soil samples (029902-0-A and 029902-0-B) were collected and analyzed for TPH and SVOCs. Results for both samples showed TPH lower than the state of Nevada action level and SVOCs lower than the Region 9 PRGs for industrial soils (NAC, 2000; EPA, 1996a), verifying that the site was clean-closed. Analytical results for the verification samples are given in Tables 2 and 3. All closure activities were documented with photographs and field notes. No further actions are required at this site.

\subsubsection{CAS 02-99-03: Epoxy Tar Spills}

The epoxy tar spills were removed from the site on August 20, 2001. The epoxy tar was placed into an end dump using a front-end loader and taken to the Area 6 Hydrocarbon Landfill for disposal. Closure activities were documented with photographs. Verification soil samples (029903-0-A, B, C and D) were collected and analyzed for TPH and SVOCs. Results showed TPH lower than the state of Nevada action level and SVOCs lower than the Region 9 PRGs for industrial soils (NAC, 2000; EPA, 1996a), verifying that the site was clean-closed. Analytical results for the verification samples are given in Tables 2 and 3. Elevated gamma spectroscopy readings were associated with the soils incorporated in the waste characterization sampling. Process knowledge indicates that the epoxy tar is not radioactive. It was not the intent of this housekeeping activity to verify whether the remaining radioactivity in the soil was still elevated since the radioactivity is not associated with the epoxy tar, but rather the nuclear testing conducted in the area. All closure activities were documented with photographs and field notes. No further actions are required at this site.

\subsubsection{CAS 02-99-04: Spill}

On August 20, 2001, concrete/cement, metal fence posts, and wood were placed into an end dump using a front-end loader and taken to the Area 9 U10c Landfill. All closure activities were documented with photographs and field notes. Clean closure of this site was verified by visual inspection; no verification samples were required or collected. No further action is required at this site.

\subsubsection{CAS 02-99-11: Spill}

The epoxy spill was removed from the site on August 10,2001. The epoxy was placed into an end dump using a front-end loader and taken to the Area 6 Hydrocarbon Landfill for disposal. Closure activities were documented with photographs. Verification soil samples (029911-0-A, - 
B, C, D, and E) were collected and analyzed for TPH. Results for all samples showed TPH lower than the state of Nevada action level (NAC, 2000), verifying that the site was clean-closed. Analytical results for the verification samples are given in Table 3. All closure activities were documented with photographs and field notes. No further actions are required at this site.

\subsubsection{CAS 03-22-22: Bucket; Spill}

On August 20, 2001, the dark soil and debris, including steel cables, drum lids, electrical debris, bolts, and wood, were placed into an end dump using a front-end loader and taken to the Area 9 U10c Landfill for disposal. All closure activities were documented with photographs and field notes. Clean closure of this site was verified by visual inspection; no verification samples were required or collected. No further action is required at this site.

\subsubsection{CAS 03-44-03: Spills}

The two spills that stained the soil were removed from the site on August 20, 2001. The stained soil was placed into an end dump using a front-end loader and taken to the Area 6 Hydrocarbon Landfill for disposal. Closure activities were documented with photographs. Verification soil samples (034403-0-A and 034403-0-B) were collected and analyzed for TPH. Results for both samples showed TPH lower than the state of Nevada action level (NAC, 2000), verifying that the site was clean-closed. Analytical results for the verification samples are given in Table 3. All closure activities were documented with photographs and field notes. No further actions are required at this site.

\subsubsection{CAS 03-99-01: Spill}

The epoxy tar spill and metal debris were removed from the site on July 25, 2001. The epoxy and metal debris were placed into an end dump using a front-end loader and taken to the Area 6 Hydrocarbon Landfill for disposal. Verification soil samples (039901-0-A and 0399010-B) were collected and analyzed for TPH and SVOCs. Results showed TPH lower than the state of Nevada action level and SVOCs lower than the Region 9 PRGs for industrial soils (NAC, 2000; EPA, 1996a), verifying that the site was clean-closed. Analytical results for the verification samples are given in Tables 2 and 3 . All closure activities were documented with photographs and field notes. No further actions are required at this site.

\subsubsection{CAS 03-99-03: Spill}

The epoxy tar spill and debris, which included a trash can lid and an empty paint can, were removed from the site on July 24, 2001. The epoxy tar and debris were placed into an end dump using a front-end loader and taken to the Area 6 Hydrocarbon Landfill for disposal. Verification soil samples (039903-0-A) were collected and analyzed for TPH and SVOCs. Results showed TPH lower than the state of Nevada action level and SVOCs lower than the Region 9 PRGs for industrial soils (NAC, 2000; EPA, 1996a), verifying that the site was clean-closed. Analytical results for the verification samples are given in Tables 2 and 3 . All closure activities were documented with photographs and field notes. No further actions are required at this site. 
TABLE 2 - SUMMARY OF ANALYTICAL RESULTS FOR SVOC VERIFICATION SAMPLES

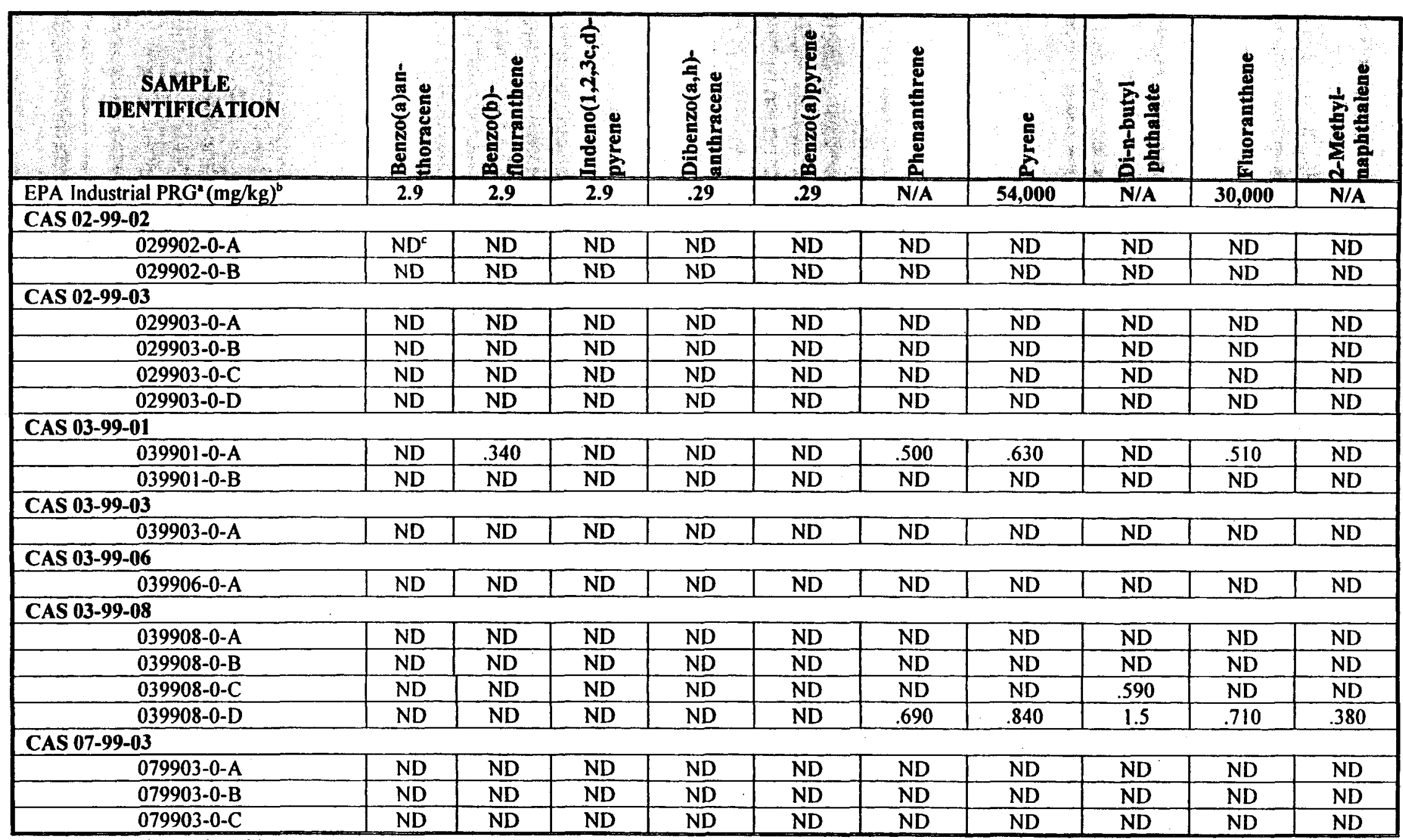

Note: All samples analyzed by U.S. Environmental Protection Agency (EPA) Method SW-846 8270 (EPA, 1996b). See analytical reports in Appendix A for detection limits.

EPA Region IX Industrial Preliminary Remediation Goals (PRGs) for 1996 (EPA, 1996a). Some PRGs have no preliminary remediation goals.

${ }^{b} \mathrm{mg} / \mathrm{kg}=$ Milligram per kilogram.

$N D=$ Not detected at the laboratory reporting limit. 


\section{TABLE 3 - SUMMARY OF ANALYTICAL RESULTS FOR TOTAL PETROLEUM HYDROCARBONS IN VERIFICATION SAMPLES}

\begin{tabular}{|c|c|c|c|c|}
\hline SAMPLEIDENTIFICATION & $\begin{array}{l}\text { GASOLINE } \\
\text { RANGE } \\
\text { (mgAg) }\end{array}$ & $\begin{array}{l}\text { DIESEL } \\
\text { RANGE } \\
\text { (mg/kg) }\end{array}$ & $\begin{array}{c}\text { OIL } \\
\text { RANGE } \\
\text { (mgkg) }\end{array}$ & $\begin{array}{l}\text { TOTAL PETROLEUM } \\
\text { HYDROCARBONS } \\
\text { (mg/kg) }\end{array}$ \\
\hline NDEP" Action Level (mg/kg) & 100 & 100 & 100 & 100 \\
\hline \multicolumn{5}{|l|}{ CAS 02-99-02 } \\
\hline $029902-0-A$ & $\mathrm{ND}^{\mathrm{c}}$ & 48 & ND & 48 \\
\hline 029902-0-B & ND & 32 & ND & 32 \\
\hline \multicolumn{5}{|l|}{ CAS 02-99-03 } \\
\hline 029903-0-A & ND & ND & ND & ND \\
\hline 029903-0-B & ND & ND & $\mathrm{ND}$ & ND \\
\hline 029903-0-C & ND & ND & ND & ND \\
\hline 029903-0-D & ND & ND & ND & ND \\
\hline \multicolumn{5}{|l|}{ CAS 02-99.11 } \\
\hline 029911-0-A & ND & ND & ND & ND \\
\hline 029911-0-B & ND & ND & ND & ND \\
\hline 029911-0-C & ND & ND & ND & ND \\
\hline $029911-0-D$ & ND & ND & ND & ND \\
\hline 029911-0-E & ND & ND & ND & ND \\
\hline \multicolumn{5}{|l|}{ CAS 03-99-01 } \\
\hline $039901-0-A$ & ND & $\mathrm{ND}$ & ND & ND \\
\hline 039901-0-B & ND & ND & ND & ND \\
\hline \multicolumn{5}{|l|}{ CAS 03-99-03 } \\
\hline 039903-0-A & ND & ND & ND & ND \\
\hline \multicolumn{5}{|l|}{ CAS 03-99-06 } \\
\hline 039906-0-A & ND & 25 & ND & 25 \\
\hline \multicolumn{5}{|l|}{ CAS 03-44-03 } \\
\hline $034403-0-\mathrm{A}$ & ND & ND & ND & ND \\
\hline 034403-0-B & ND & ND & ND & ND \\
\hline \multicolumn{5}{|l|}{ CAS 03-99-08 } \\
\hline 039908-0-A & ND & ND & ND & $\mathrm{ND}$ \\
\hline 039908-0-B & ND & ND & $\overline{\text { ND }}$ & ND \\
\hline 039908-0-C & ND & 22 & ND & 22 \\
\hline 039908-0-D & ND & ND & $\overline{\mathrm{ND}}$ & ND \\
\hline \multicolumn{5}{|l|}{ CAS 07-99-03 } \\
\hline 079903-0-A & ND & ND & ND & ND \\
\hline 079903-0-B & ND & ND & $\mathrm{ND}$ & ND \\
\hline 079903-0-C & ND & ND & ND & ND \\
\hline
\end{tabular}

"NDEP-Nevada Division of Environmental Protection

${ }^{\mathrm{b}} \mathrm{mg} . \mathrm{kg}$-Milligrams per kilogram

TD-Not detected for the laboratory reporting limits. 


\subsubsection{CAS 03-99-06: Spill}

The epoxy tar spill was removed from the site on July 25,2001 , by placing material into an end dump using a front-end loader and taking it to the Area 6 Hydrocarbon Landfill for disposal. Verification soil samples (039906-0-A) were collected and analyzed for TPH and SVOCs. Results showed TPH lower than the state of Nevada action level and SVOCs lower than the Region 9 PRGs for industrial soils (NAC, 2000; EPA, 1996a), verifying that the site was cleanclosed. Analytical results for the verification samples are given in Tables 2 and 3. All closure activities were documented with photographs and field notes. No further actions are required at this site.

\subsubsection{CAS 03-99-08: Tar Spill}

The tar spill was removed from the site on August 17, 2001. The tar was placed into B-25 containers due to its potential to be LLW. Containers are currently being further characterized for LLW. Verification soil samples (039908-0-A, B, C, and D) were collected and analyzed for TPH and SVOCs. Results showed TPH lower than the state of Nevada action level and SVOCs lower than the Region 9 PRGs for industrial soils (NAC, 2000; EPA, 1996a), verifying that the site was clean-closed. Analytical results for the verification samples are given in Tables 2 and 3. Elevated gamma spectroscopy readings were associated with the soils incorporated in the waste characterization sampling. Process knowledge indicates that the tar is not radioactive. It was not the intent of this housekeeping activity to verify whether the remaining radioactivity in the soil was still elevated since the radioactivity is not associated with the tar, but rather the nuclear testing conducted in the area. All closure activities were documented with photographs and field notes. No further actions are required at this site.

\subsubsection{CAS 03-99-17: Bottle Spill}

The amber glass bottle and scrap metal were removed on August 20, 2001. No soil verification sampling was needed at this site. No COCs were found in the waste characterization soil samples collected from beneath the bottle or the bottle and its liquid contents. All closure activities were documented with photographs and field notes. No further actions are required at this site.

\subsubsection{CAS 03-99-18: Cement Spill}

The cement spill was removed on August 17, 2001. The cement was placed into an end dump using a back hoe and taken to the Area 9 U10c Landfill for disposal. All closure activities were documented with photographs and field notes. Clean closure of this site was verified by visual inspection; no verification samples were required or collected. No further action is required at this site.

\subsubsection{CAS 04-44-01: Spill}

The cement/grout material and debris, which included wire, rope, and wood, were removed on August 21,2001. The cement/grout material and debris were placed into an end dump using a front-end loader and taken to the Area 9 U10c Landfill for disposal. All closure activities were documented with photographs and field notes. Clean closure of this site was verified by visual 
inspection; no verification samples were required or collected. No further action is required at this site.

\subsubsection{CAS 04-44-03: U-4t Postshot Hole Release}

The drilling/grout material was removed on August 21,2001, by placing material into an end

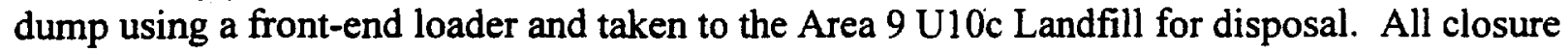
activities were documented with photographs and field notes. Clean closure of this site was verified by visual inspection; no verification samples were required or collected. No further action is required at this site.

\subsubsection{CAS 07-99-02: Discolored Area}

The pile of discolored cement and numerous pieces of debris, which included metal, wire, rubber, and wood, were removed on August 3,2001. The discolored cement and debris were placed into an end dump using a front-end loader and taken to the Area $9 \mathrm{U} 10 \mathrm{c}$ Landfill for disposal. All closure activities were documented with photographs and field notes. Clean closure of this site was verified by visual inspection; no verification samples were required or collected. No further action is required at this site.

\subsubsection{CAS 07-99-03: Compressed Bottle; Tar Spill}

The two small tar spills and debris, which included a wooden spool and wire, were removed on August 3, 2001, by placing material and debris into an end dump using a front-end loader and taking it to the Area 6 Hydrocarbon Landfill for disposal. Verification soil samples (079903-0A, B, and C) were collected and analyzed for TPH and SVOCs. Results showed TPH lower than the state of Nevada action level and SVOCs lower than the Region 9 PRGs for industrial soils (NAC, 2000; EPA, 1996a), verifying that the site was clean-closed. Analytical results for the verification samples are given in Tables 2 and 3. On September 25, 2001, the gas cylinder was neutralized with explosives and taken to the Area 9 U10c Landfill for disposal. All closure activities were documented with photographs and field notes. No further actions are required at this site. 


\section{THIS PAGE INTENTIONALLY LEFT BLANK}




\subsection{WASTE DISPOSITION}

Wastes generated during the closure of CAU 387: Spill Sites and Releases were disposed as follows:

- CAS 02-99-02: approximately 7.7 cubic meters $\left(\mathrm{m}^{3}\right)$ (10 cubic yards [yd $\left.\left.\mathrm{d}^{3}\right]\right)$ of epoxy tar/soil and debris placed into B-25 containers as potential LLW. Contents of the B-25 containers will be sampled for radioisotopes and disposed of accordingly.

- CAS 02-99-03: approximately $1.5 \mathrm{~m}^{3}\left(2 \mathrm{yd}^{3}\right)$ of epoxy tar/soil disposed of in the Area 6 Hydrocarbon Landfill.

- CAS 02-99-04: approximately $25.4 \mathrm{~m}^{3}\left(33 \mathrm{yd}^{3}\right)$ of concrete/cement and debris disposed of as sanitary waste in the Area $9 \mathrm{U} 10 \mathrm{c}$ Landfill.

- CAS 02-99-11: approximately $2.3 \mathrm{~m}^{3}\left(3 \mathrm{yd}^{3}\right)$ of epoxy/soil disposed in the Area 6 Hydrocarbon Landfill.

- CAS 03-22-22: approximately $3.8 \mathrm{~m}^{3}\left(5 \mathrm{yd}^{3}\right)$ of soil and debris disposed of as sanitary waste in the Area 9 U10c Landfill.

- CAS 03-44-03: approximately $1.5 \mathrm{~m}^{3}\left(2 \mathrm{yd}^{3}\right)$ of TPH-impacted soil disposed of in the Area 6 Hydrocarbon Landfill.

- CAS 03-99-01: approximately $1.5 \mathrm{~m}^{3}\left(2 \mathrm{yd}^{3}\right)$ of epoxy tar and scrap metal disposed of in the Area 6 Hydrocarbon Landfill.

- CAS 03-99-03: approximately $1.5 \mathrm{~m}^{3}\left(2 \mathrm{yd}^{3}\right)$ of epoxy tar and debris disposed of in the Area 6 Hydrocarbon Landfill.

- CAS 03-99-06: approximately $2.3 \mathrm{~m}^{3}\left(3 \mathrm{yd}^{3}\right)$ of epoxy tar disposed of in the Area 6 Hydrocarbon Landfill.

- CAS 03-99-08: approximately $12.3 \mathrm{~m}^{3}\left(16 \mathrm{yd}^{3}\right)$ of tar placed into B-25 containers as potential LLW. Contents of the B-25 containers will be sampled for radioisotopes and disposed of accordingly.

- $\quad$ CAS 03-99-17: amber glass bottle with contents sent to laboratory for analysis; scrap metal disposed of as sanitary waste in the Area 9 U10c Landfill.

- CAS 03-99-18: approximately $4.6 \mathrm{~m}^{3}\left(6 \mathrm{yd}^{3}\right)$ of cement disposed of as sanitary waste in the Area 9 U10c Landfill.

- CAS 04-44-01: approximately $7.7 \mathrm{~m}^{3}\left(10 \mathrm{yd}^{3}\right)$ of cement/grout material and debris disposed of as sanitary waste in the Area $9 \mathrm{U} 10 \mathrm{c}$ Landfill. 
- CAS 04-44-03: approximately $19 \mathrm{~m}^{3}\left(25 \mathrm{yd}^{3}\right)$ of drilling/grout material disposed of as sanitary waste in the Area 9 U10c Landfill.

- CAS 07-99-02: approximately $1.5 \mathrm{~m}^{3}\left(2 \mathrm{yd}^{3}\right)$ of discolored cement and debris disposed of as sanitary waste in the Area 9 U10c Landfill.

- CAS 07-99-03: approximately $1.5 \mathrm{~m}^{3}\left(2 \mathrm{yd}^{3}\right)$ of epoxy tar and debris disposed of in the Area 6 hydrocarbon landfill; gas cylinder disposed as sanitary waste in the Area 9 U10c Landfill. 


\subsection{CLOSURE VERIFICATION}

The following nine CASs required verification soil sampling:

$$
\begin{array}{ll}
- & 02-99-02 \\
\text { - } & 02-99-03 \\
\text { - } & 02-99-11 \\
\text { - } & 03-44-03 \\
\text { - } & 03-99-01 \\
\text { - } & 03-99-03 \\
\text { - } & 03-99-06 \\
\text { - } & 03-99-08 \\
\text { - } & 07-99-03
\end{array}
$$

Samples were collected from a total of 24 locations after the excavations reached boundaries determined by field screening and/or no visible staining was observed. The samples were collected with clean disposable plastic scoops and placed in labeled sample containers secured with custody seals. The sample containers were placed on ice in a cooler; transported under chain of custody to the BN Sample Management group in Mercury, Nevada; and shipped to an off-site laboratory for analysis. Samples from 17 locations were analyzed for SVOCs (EPA, 1996a). Samples from 23 locations were analyzed for TPH (EPA, 1996b). Some locations required both, TPH and SVOC verification soil samples be collected.

The analytical results verify that SVOCs and TPH remaining in the ground at the nine sites are below EPA Region 9 PRGs for industrial soil (EPA, 1996a), and the state of Nevada action level for TPH (i.e., $100 \mathrm{mg} / \mathrm{kg}$ ) (NAC, 2000). The analytical results for SVOCs and TPH are summarized in Tables 2 and 3, respectively; the analytical reports are included in Appendix A. 
CLOSURE REPORT - CAU 387

Spill Sites and Releases

Section: Closure Verification

Revision: 0

Date: October 2001

THIS PAGE INTENTIONALLY LEFT BLANK 


\subsection{SUMMARY AND RECOMMENDATIONS}

\subsection{SUMMARY}

The following site closure activities were performed at the 16 of the 23 CASs comprising CAU 387 and are documented in the report:

- All debris (e.g., wood, metal cable, scrap metal, rope, electrical debris, drum lids, wire, empty cans, rubber, and/or gas cylinders) were removed from the sites and disposed in either the Area 9 U10c Landfill or the Area 6 Hydrocarbon Landfill, depending upon its type.

- $\quad$ All soil containing TPH levels at or above the state of Nevada TPH action level (i.e., $100 \mathrm{mg} / \mathrm{kg}$ ) (NAC, 2000) was removed and disposed in the NTS Area 6 Hydrocarbon Landfill.

- All material, including soil, containing cement or drilling/grout material was removed from the sites and disposed in the Area 9 U10c Landfill.

\subsection{RECOMMENDATIONS}

Since the closure activities for CAU 387 have been completed following the Nevada Division of Environmental Protection (NDEP)-approved Sectored Clean-up Work Plan for Housekeeping Category Waste Sites (DOE/NV, 2000) as documented in this report, the U.S. Department of Energy, National Nuclear Security Administration Nevada Operations Office (NNSA/NV) requests the following:

- A Notice of Completion be provided by the NDEP to the NNSANNV for the closure of CAU 387 (CAS 02-99-02, CAS 02-99-03, CAS 02-99-04, CAS 02-99-07, CAS 02-99-09, CAS 02-99-11, CAS 02-44-01, CAS 03-22-22, CAS 03-22-35, CAS 03-44-03, CAS 0399-01, CAS 03-99-02, CAS 03-99-03, CAS 03-99-06, CAS 03-99-07, CAS 03-99-08, CAS 03-99-09, CAS 03-99-17, CAS 03-99-18, CAS 04-44-01, CAS 04-44-03, CAS 07-99-02, and CAS 07-99-03).

CAU 387 be moved from Appendix III to Appendix IV of the FFACO Closed Corrective Action Units (FFACO, 1996). 
THIS PAGE INTENTIONALLY LEFT BLANK 


\subsection{REFERENCES}

BN, see Bechtel Nevada.

Bechtel Nevada. 2000. Field Management Plan for Housekeeping Category Waste Sites, Las Vegas, NV.

Bechtel Nevada. 2001. Site-Specific Health and Safety Plan for Corrective Action Unit 387: Areas 2, 3, 4 and 7 Spill Sites and Releases, Nevada Test Site, Nevada, Las Vegas, NV.

DOE/NV, see U.S. Department of Energy, Nevada Operations Office.

EPA, see U.S. Environmental Protection Agency.

FFACO, see Federal Facility Agreement and Consent Order.

Federal Facility Agreement and Consent Order of 1996. Agreed to by the Nevada Division of Environmental Protection, U.S. Department of Energy, and U.S. Department of Defense.

Liebendorfer, Paul J. 2001. Letter, "Proposed Modifications to the Remediation Process for Various CASs, Corrective Action Unit (CAU) 387, Federal Facility Agreement and Consent Order," September 28, 2001.

NAC, see Nevada Administrative Code.

Nevada Administrative Code. 2000. NAC 445A.2272, Contamination of soil: Establishment of action levels. As adopted by the Nevada Environmental Commission, October, Carson City, NV.

U.S. Department of Energy, Nevada Operations Office. 2000. Sectored Clean-up Work Plan for Housekeeping Category Waste Sites, Rev 0, DOE/NV--579, Las Vegas, NV.

U.S. Environmental Protection Agency. 1996a. Region LX Preliminary Remediation Goals (PRGs), San Francisco, CA.

U.S. Environmental Protection Agency. 1996b. Test Methods for Evaluating Solid Waste, Physical/Chemical Methods, EPA Publication SW-846, Third Edition. Washington, D.C. 
CLOSURE REPORT - CAU 387

Spill Sites and Releases

Section: References

Revision: 0

Date: October 2001

\section{THIS PAGE INTENTIONALLY LEFT BLANK}




\section{APPENDIX A VERIFICATION SAMPLE ANALYTICAL RESULTS}


a

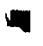

n

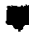

THIS PAGE INTENTIONALLY LEFT BLANK

a 
$\begin{array}{ll}\text { CLIENT: } & \text { Bechtel Nevada } \\ & \text { P.O. Box 98521, M/S NTS273 } \\ & \text { Las Vegas, NV 89193-8521 } \\ \text { ATTN: } & \text { Ted Redding }\end{array}$

PROJECT NAME: $\quad$ V1243

PROJECT NUMBER: 23081
NEL ORDER ID: L0108210

Attached are the analytical results for samples in support of the above referenced project.

Samples submitted for this project were not sampled by NEL Laboratories. Samples were received by NEL in good condition, under chain of custody on 8/21/01.

Should you have any questions or comments, please feel free to contact our Client Services department at (702) 657-1010.

\section{Some results have been flagged as follows:}

$\mathrm{Di}$ - Results reported from analysis at a higher dilution.

J - This concentration should be considered an estimate due laboratory control sample failure.

Jl - The batch MS and/or MSD were outside acceptance limits. The batch LCS was acceptable.

Some $Q A$ results have been flagged as follows:

J - This concentration should be considered an estimate due laboratory control sample failure.

Jl - The batch MS and/or MSD were outside acceptance limits. The batch LCS was acceptable.

R5 - RPD exceeded the laboratory control limit. Recovery met acceptance criteria.

Some surrogate results have been flagged as follows:

Sf - This surrogate was outside acceptance limits.
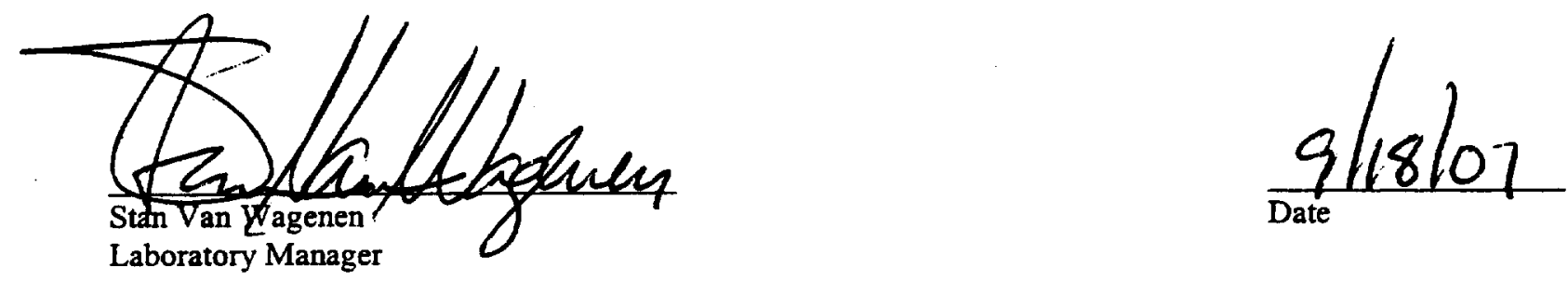

\section{CERTIFICATIONS:}

\begin{tabular}{llll}
\hline & Reno & Las Vegas & S. Califormia \\
\cline { 2 - 4 } $\begin{array}{l}\text { Arizona } \\
\text { California }\end{array}$ & 170720 & AZ0518 & AZ0605 \\
$\begin{array}{l}\text { US Army Corps } \\
\text { of Engineers }\end{array}$ & Certified & Certified & 2264 \\
& & &
\end{tabular}

\begin{tabular}{llll} 
& Reno & Las Vegas & S. California \\
\cline { 2 - 4 } Idaho & Certified & Certified & \\
Montana & Certified & Certified & \\
Nevada & NV033 & NV052 & CA084 \\
L.A.C.S.D. & & & 10228
\end{tabular}




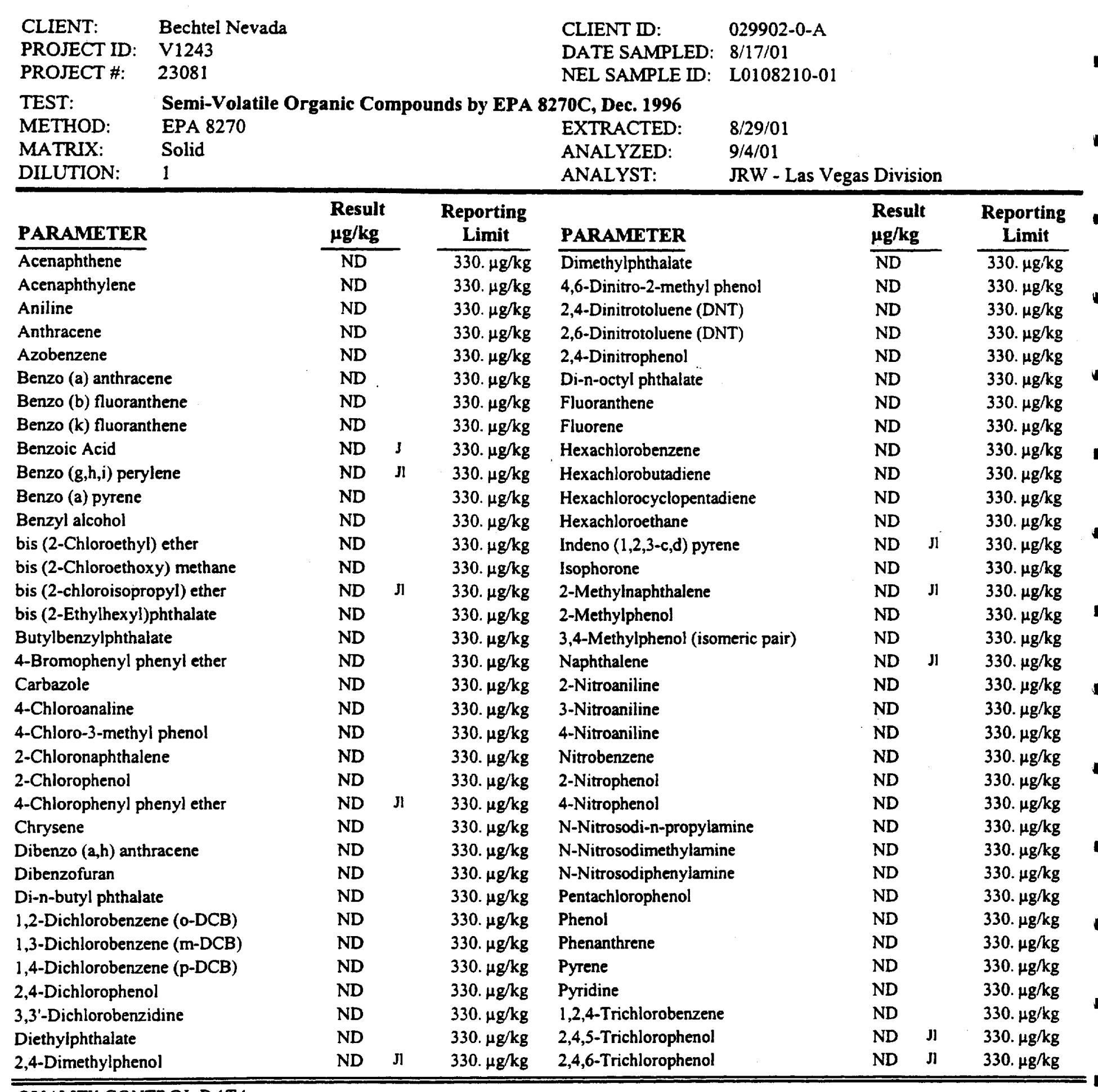

QUALITY CONTROL DATA:

\section{Surrogate}

2,4,6-Tribromophenol

2-Fluorobiphenyl

2-Fluorophenol

Nitrobenzene-d5

p-Terphenyl-d14

Phenol-d6

\section{$\%$ Recovery}

52

50

38

39

76

49
Acceptable Range

$19-122$

$30-115$

$25-121$

$23-120$

$18-137$

$24-113$

ND - Not Detected

This report shall not be reproduced except in full, without the written approval of the laboratory. 


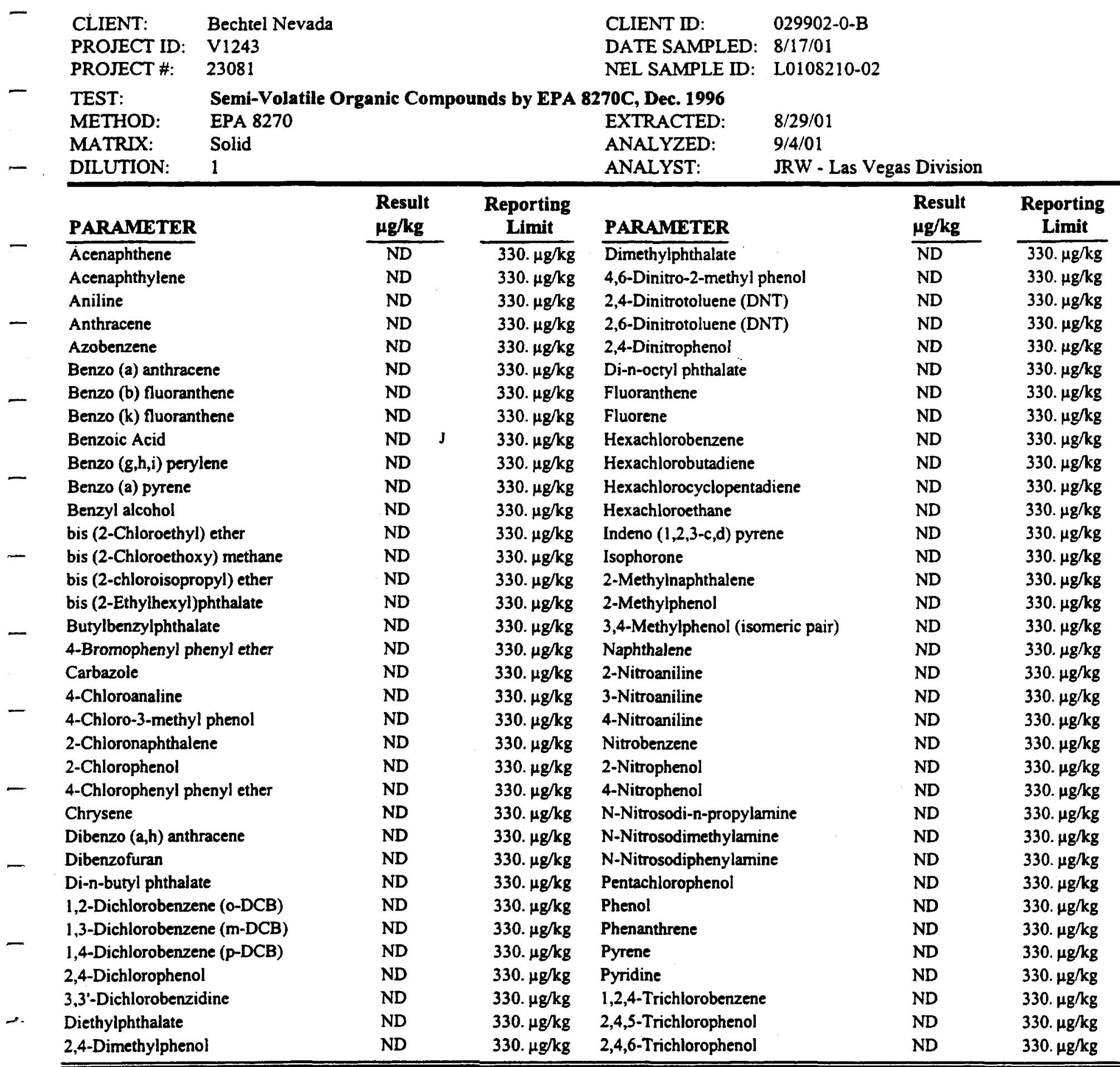

QUALITY CONTROL DATA:

\section{Surrogate}

2,4,6-Tribromophenol

2-Fluorobiphenyl

2-Fluorophenol

Nitrobenzene-d5

p-Terphenyl-d14

Phenol-d6
\% Recovery

64

59

44

43

66

51

\section{Acceptable Range}

$19-122$

$30-115$

$25-121$

$23-120$

$18-137$

$24-113$

ND - Not Detected

This report shall not be reproduced except in full, without the written approval of the laboratory. 


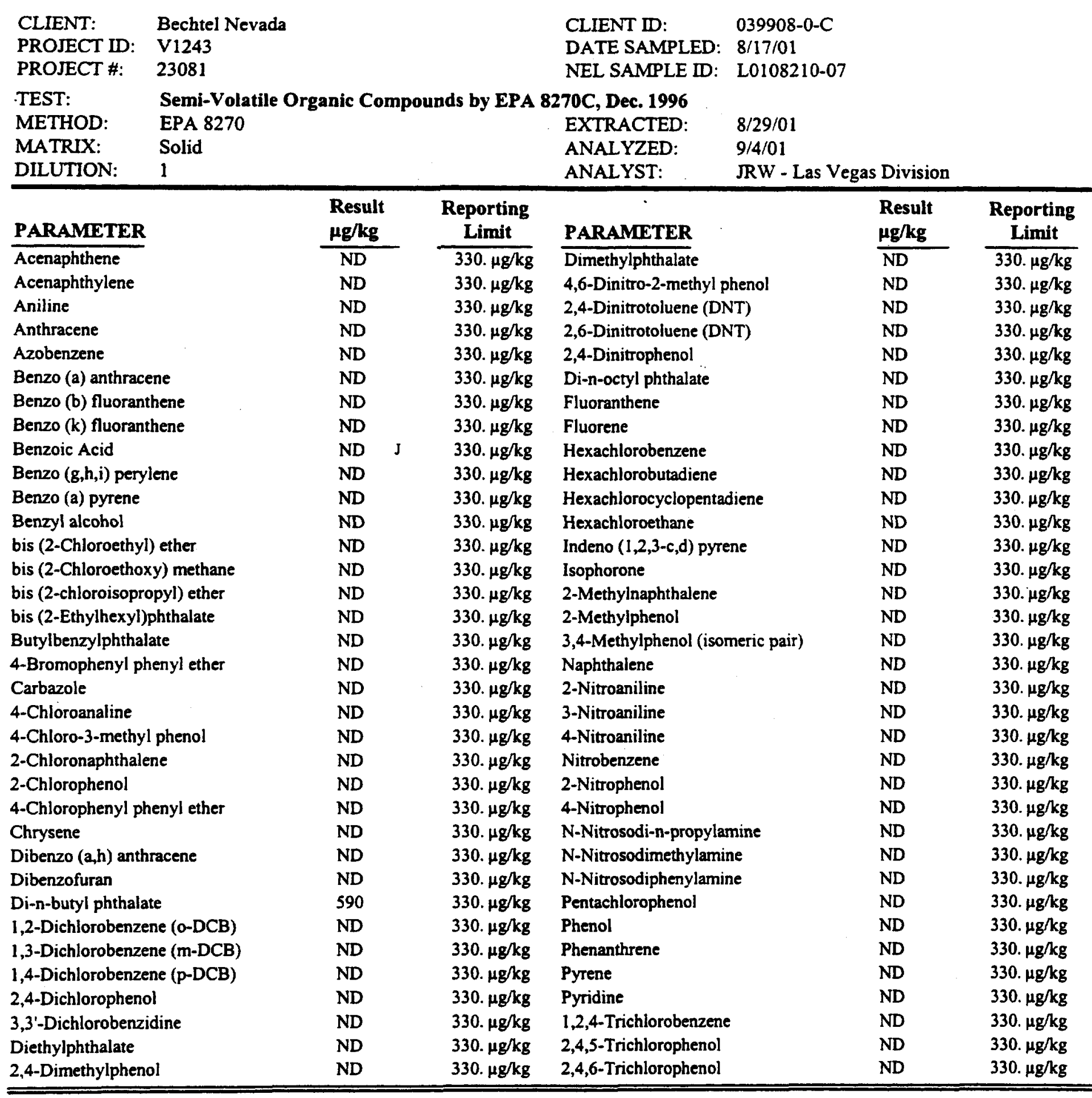

QUALITY CONTROL DATA:

\section{Surrogate}

2,4,6-Tribromophenol

2-Fluorobiphenyl

2-Fluorophenol

Nitrobenzene-d5

p-Terphenyl-d14

Phenol-d6

\begin{tabular}{c} 
\% Recovery \\
\hline 51 \\
46 \\
39 \\
39 \\
67 \\
44
\end{tabular}

\section{Acceptable Range}

$19-122$

$30-115$

$25-121$

$23-120$

$18-137$

$24-113$

ND - Not Detected

This report shall not be reproduced except in full, without the written approval of the laboratory. 


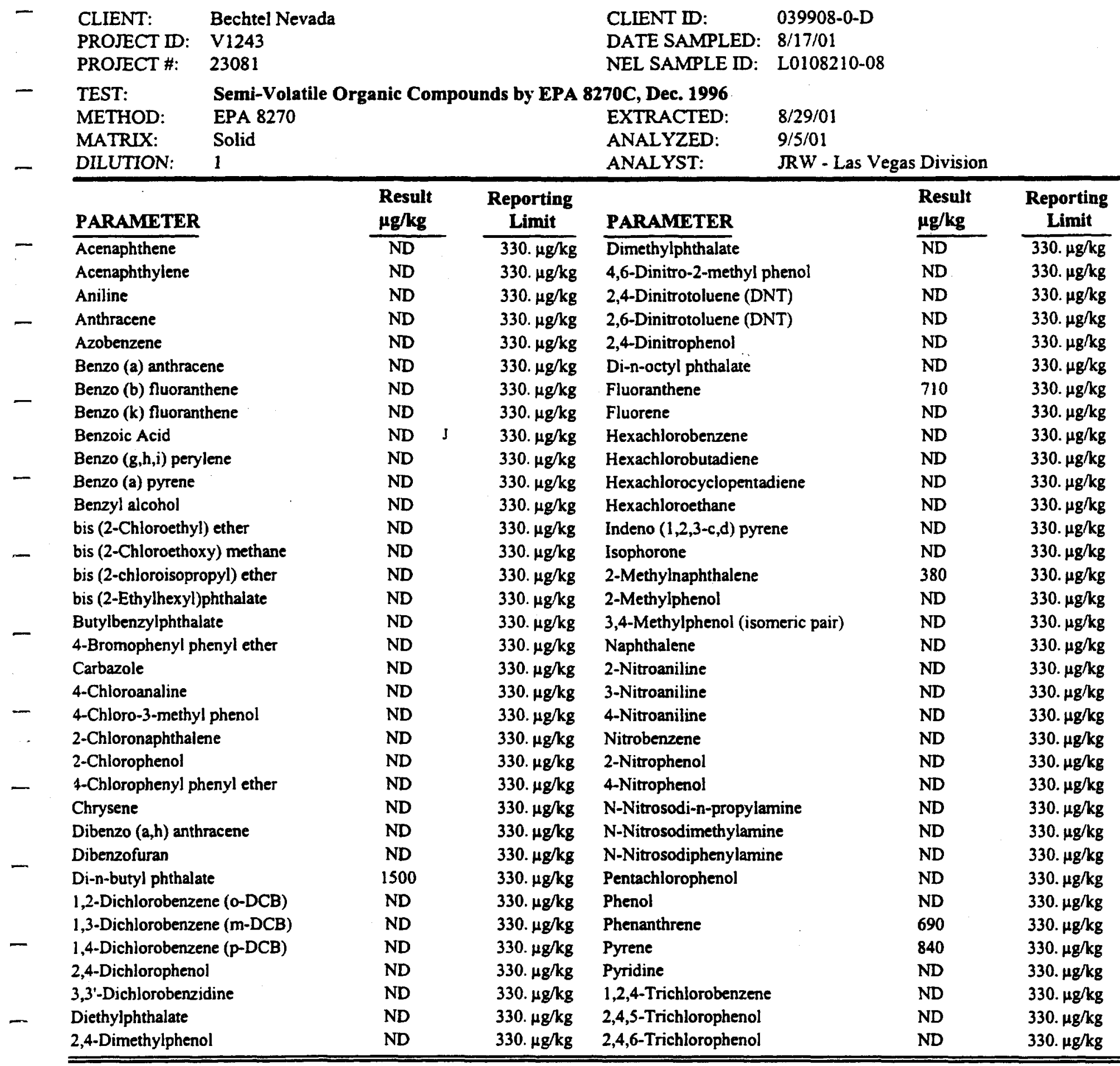

QUALITY CONTROL DATA:

\section{Surrogate}

2,4,6-Tribromophenol

2-Fluorobiphenyl

2-Fluorophenol

Nitrobenzene-d5

p-Terphenyl-d14

Phenol-d6

\section{$\%$ Recovery}

69

71

53

58

83

61
Acceptable Range

$19-122$

$30-115$

$25-121$

$23-120$

$18-137$

$24-113$

ND - Not Detected

This report shall not be reproduced except in full, without the written approval of the laboratory. 


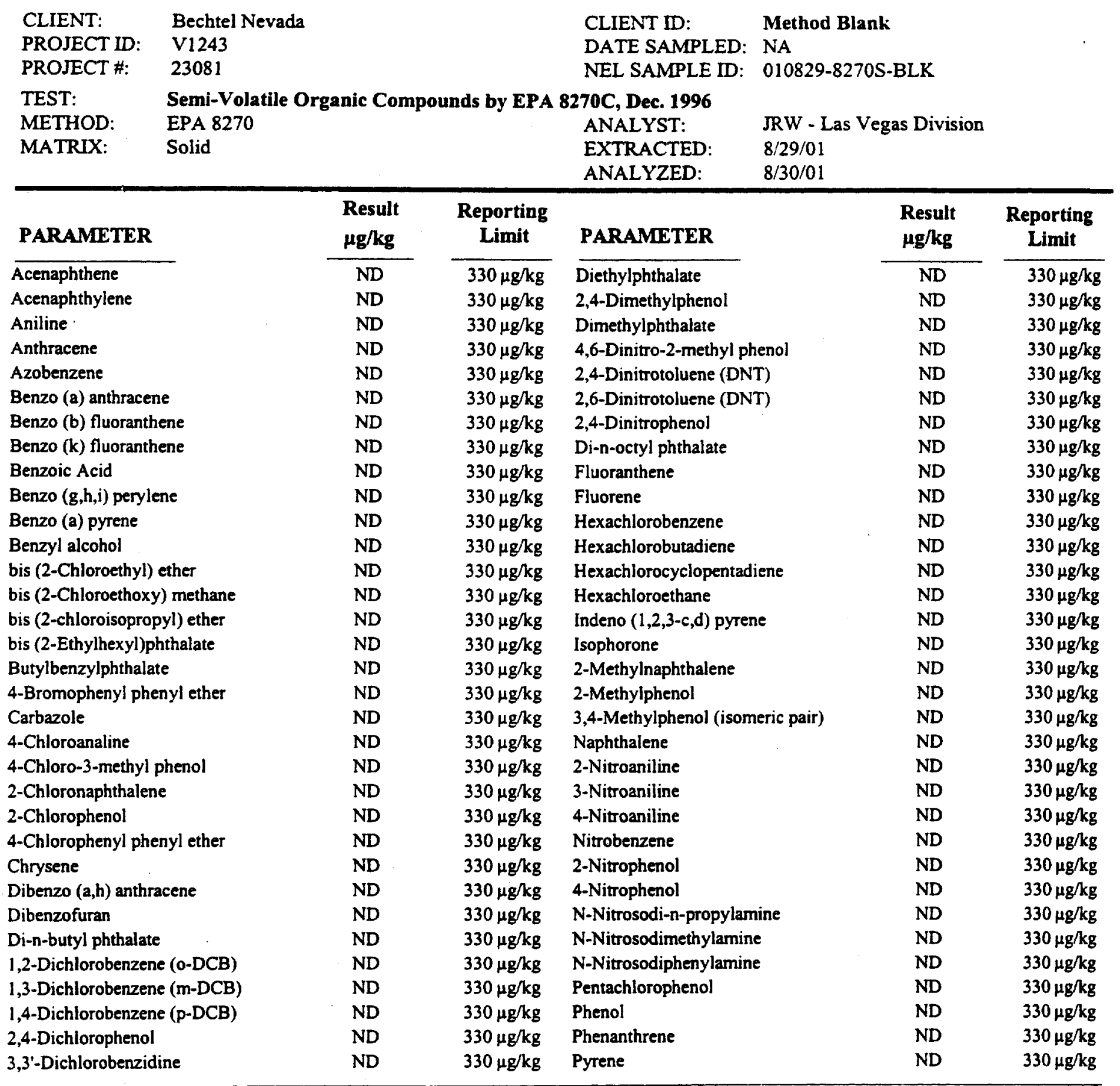

QUALITY CONTROL DATA:

\section{Surrogate}

2,4,6-Tribromophenol

2-Fluorobiphenyl

2-Fluorophenol

Nitrobenzene-d5

p-Terphenyl-d14

Phenol-d6

\begin{tabular}{c} 
\% Recovery \\
\hline 51 \\
54 \\
53 \\
55 \\
66 \\
56
\end{tabular}

Acceptable Range

$19-122$

$30-115$

$25-121$

$23-120$

$18-137$

$24-113$

ND - Not Detected

This report shall not be reproduced except in full, without the written approval of the laboratory. 
NEL LABORATORIES

\begin{tabular}{|c|c|c|}
\hline $\begin{array}{l}\text { CLIENT: } \\
\text { PROJECT ID: } \\
\text { PROJECT \#: }\end{array}$ & $\begin{array}{l}\text { Bechtel Nevada } \\
\text { V1243 } \\
23081\end{array}$ & $\begin{array}{ll}\text { CLIENT ID: } & \mathbf{0 2 9 9 0 2 - 0 - A} \\
\text { DATE SAMPLED: } & 8 / 17 / 01 \\
\text { NEL SAMPLE ID: } & \text { L0108210-01 }\end{array}$ \\
\hline
\end{tabular}

- TEST: Total Extractable Petroleum Hydrocarbons Fuel Finger Print by EPA Method 8015M, July 1992

METHOD: EPA 8015M

MATRIX: Solid

ANALYST: $\quad$ PXC-Division

DILUTION: 1

EXTRACTED: $\quad 9 / 6 / 01$

ANALYZED: $\quad 9 / 6 / 01$

一

\section{PARAMETER}

Gasoline Range (C8-C12)

Diesel Range (C12-C22)

\begin{tabular}{rll}
\multicolumn{2}{c}{ Result } & \multicolumn{2}{c}{ Reporting } \\
\cline { 1 - 1 } ND & & $\frac{\text { Limit }}{10 . \mathrm{mg} / \mathrm{kg}}$ \\
48 & $\mathrm{mg} / \mathrm{kg}$ & $10 . \mathrm{mg} / \mathrm{kg}$ \\
$\mathrm{ND}$ & & $50 . \mathrm{mg} / \mathrm{kg}$ \\
48 & $\mathrm{mg} / \mathrm{kg}$ & $10 . \mathrm{mg} / \mathrm{kg}$ \\
\hline
\end{tabular}

Oil Range (C22-C34)

Total

$48 \mathrm{mg} / \mathrm{kg}$

Acceptable Range

Surrogate

Octacosane

\% Recovery

55

$54-130$

ND - Not Detected

This report shall not be reproduced except in full, without the written approval of the laboratory. 
NEL LABORATORIES

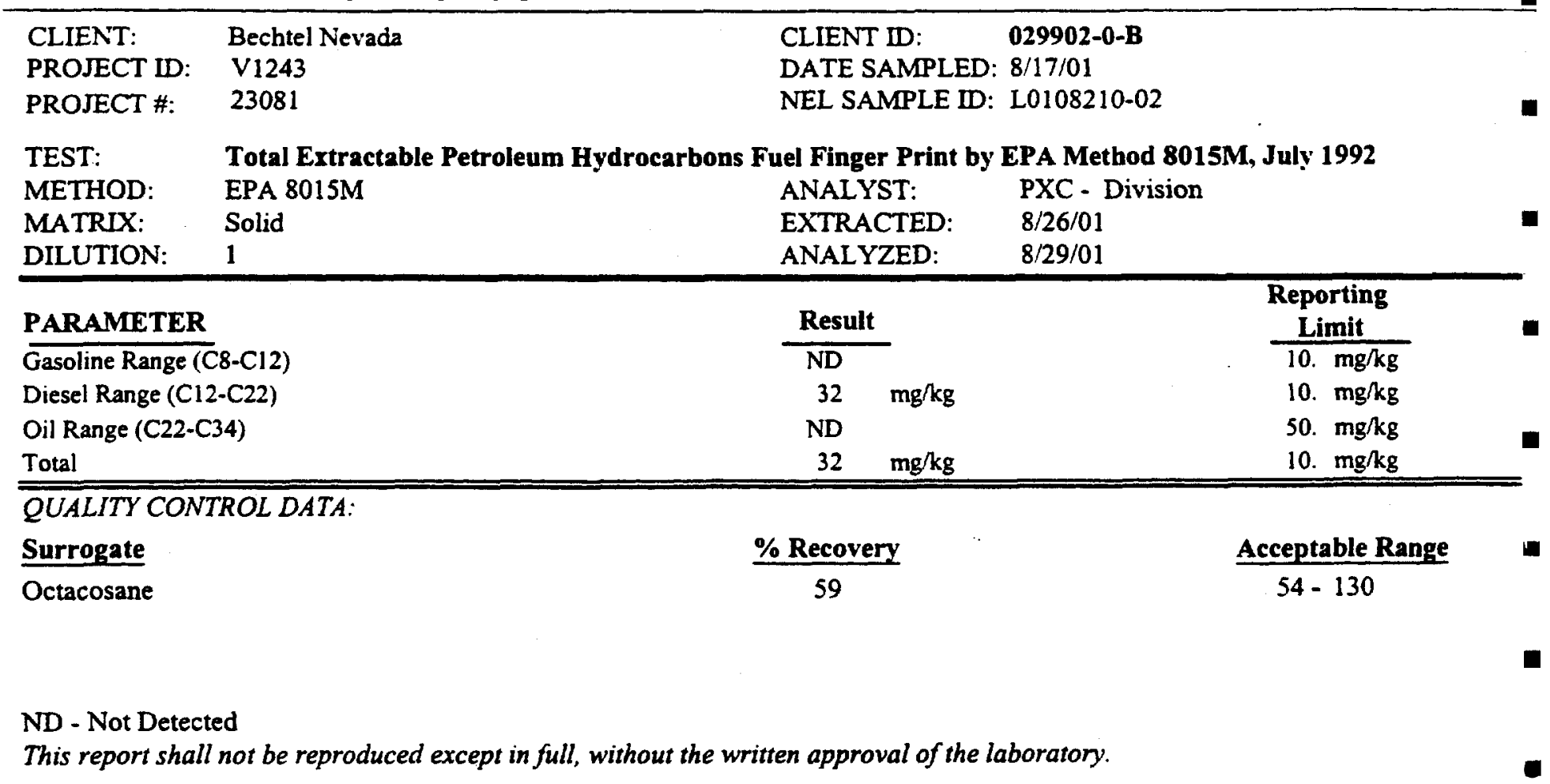


NEL LABORATORIES

\begin{tabular}{llll}
\hline CLIENT: & Bechtel Nevada & CLIENT ID: & 034403-0-A \\
PROJECT ID: & V1243 & DATE SAMPLED: $8 / 20 / 01$ \\
PROJECT \#: & 23081 & NEL SAMPLE ID: L0108210-09
\end{tabular}

- TEST: $\quad$ Total Extractable Petroleum Hydrocarbons Fuel Finger Print by EPA Method $8015 M$, July 1992

METHOD: EPA 8015M ANALYST: PXC - Division

MATRIX: Solid $\quad$ EXTRACTED: $8 / 26 / 01$

DILUTION: 1 ANALYZED: $\quad 8 / 29 / 01$

\section{PARAMETER}

Gasoline Range (C8-C12)

Diesel Range (C12-C22)

Oil Range (C22-C34)

Total

QUALITY CONTROL DATA:

Surrogate

Octacosane

\begin{tabular}{l} 
Result \\
\hline ND \\
ND \\
ND \\
ND
\end{tabular}

$\%$ Recovery

62
Reporting Limit

10. $\mathrm{mg} / \mathrm{kg}$

10. $\mathrm{mg} / \mathrm{kg}$

50. $\mathrm{mg} / \mathrm{kg}$

10. $\mathrm{mg} / \mathrm{kg}$

ND - Not Detected

This report shall not be reproduced except in full, without the written approval of the laboratory. 
NEL LABORATORIES

\begin{tabular}{|c|c|c|}
\hline CLIENT: & Bechtel Nevada & CLIENT ID: \\
\hline PROJECT ID: & V1243 & DATE SAMPLED: 8:20/01 \\
\hline PROJECT \#: & 23081 & NEL SAMPLE ID: L0108210-10 \\
\hline
\end{tabular}

TEST: $\quad$ Total Extractable Petroleum Hydrocarbons Fuel Finger Print by EPA Method 8015 M, July 1992

METHOD: $\quad$ EPA 8015M

MATRIX: Solid

ANALYST: $\quad$ PXC - Division

DILUTION: 1

ANALYZED: $\quad 8 / 29 / 01$

PARAMETER

Gasoline Range (C8-C12)

\begin{tabular}{l} 
Result \\
\hline ND \\
ND \\
ND \\
ND
\end{tabular}

Reporting

Diesel Range (C12-C22)

Oil Range (C22-C34)

Total

QUALITY CONTROL DATA:

Surrogate

\% Recovery

Octacosane

56

Acceptable Range

$54-130$

ND - Not Detected

This report shall not be reproduced except in full, without the written approval of the laboratory. 
NEL LABORATORIES

\begin{tabular}{llll}
\hline CLIENT: & Bechtel Nevada & CLIENT ID: & Method Blank \\
PROJECT ID: & V1243 & DATE SAMPLED: & NA \\
PROJECT \#: & 23081 & NEL SAMPLE ID: & 010826TPHS-3-BLK
\end{tabular}
- TEST:
METHOD: EPA 8015M
MATRIX: Solid
Total Extractable Petroleum Hydrocarbons Fuel Finger Print by EPA Method 8015M, July 1992
ANALYST: $\quad$ PXC - Division
EXTRACTED: $\quad 8 / 26 / 01$

ANALYZED: $\quad 8 / 29 / 01$

\begin{tabular}{l} 
Result \\
\hline ND \\
ND \\
ND \\
ND
\end{tabular}

\section{Reporting \\ $\frac{\text { Limit }}{10 . \mathrm{mg} / \mathrm{kg}}$ \\ 10. $\mathrm{mg} / \mathrm{kg}$ \\ 50. $\mathrm{mg} / \mathrm{kg}$ \\ 10. $\mathrm{mg} / \mathrm{kg}$}

Total

QUALITY CONTROL DATA:

Surrogate

Octacosane

$\%$ Recovery

83

Acceptable Range

$54-130$

ND - Not Detected

This report shall not be reproduced except in full, without the written approval of the laboratory.
CLIENT:
Bechtel Nevada
PROJECT ID: V1243
CLIENT ID: $\quad$ Method Blank
PROJECT \#: 23081
DATE SAMPLED: NA
NEL SAMPLE ID: 010904TPHS-1-BLK

TEST: $\quad$ Total Extractable Petroleum Hydrocarbons Fuel Finger Print by EPA Method $8015 M$, July 1992

METHOD: EPA 8015M

MATRIX: Solid

ANALYST: $\quad$ PXC - Division

EXTRACTED: $\quad 9 / 6 / 01$

ANALYZED: $\quad 9 / 6 / 01$

\section{PARAMETER}

Gasoline Range (C8-C12)

Diesel Range (C12-C22)

Oil Range (C22-C34)

Total

Surrogate

Octacosane

\begin{tabular}{l} 
Result \\
\hline ND \\
ND \\
ND \\
ND
\end{tabular}

Reporting

$\frac{\text { Limit }}{10 . \mathrm{mg} / \mathrm{kg}}$

10. $\mathrm{mg} / \mathrm{kg}$

50. $\mathrm{mg} / \mathrm{kg}$

10. $\mathrm{mg} / \mathrm{kg}$

\section{- QUALITY CONTROL DATA:}

-

ND - Not Detected

This report shall not be reproduced except in full, without the written approval of the laboratory. 
CLIENT: $\quad$ Bechtel Nevada

PROJECT ID: V1243

PROJECT \#: 23081

TEST: $\quad$ Total Extractable Petroleum Hydrocarbons Fuel Finger Print by EPA Method $8015 M$, July 1992

MATRIX: Solid

\begin{tabular}{|c|c|c|c|c|c|c|}
\hline PARAMETER & NEL Sample ID & Spike & $\frac{\text { Spike }}{\text { Result }}$ & $\frac{\text { Percent }}{\text { Recovery }}$ & $\frac{\text { Acceptable }}{\text { Range }}$ & $\underline{\mathbf{R P D}}$ \\
\hline Diesel Range (C12-C22) & 010826TPHS-3-LCS & 166.7 & 121 & 73 & $53-91$ & \\
\hline Diesel Range (C12-C22) & 010826TPHS-3-LCSD & 166.7 & 119 & 71 & $53-91$ & 1.7 \\
\hline Total & 010826TPHS-3-LCS & 166.7 & 121 & 73 & $53-91$ & \\
\hline Total & 010826TPHS-3-LCSD & 166.7 & 119 & 71 & $53-91$ & 1.7 \\
\hline
\end{tabular}

ND - Not Detected

This report shall not be reproduced except in full, without the written approval of the laboratory. 


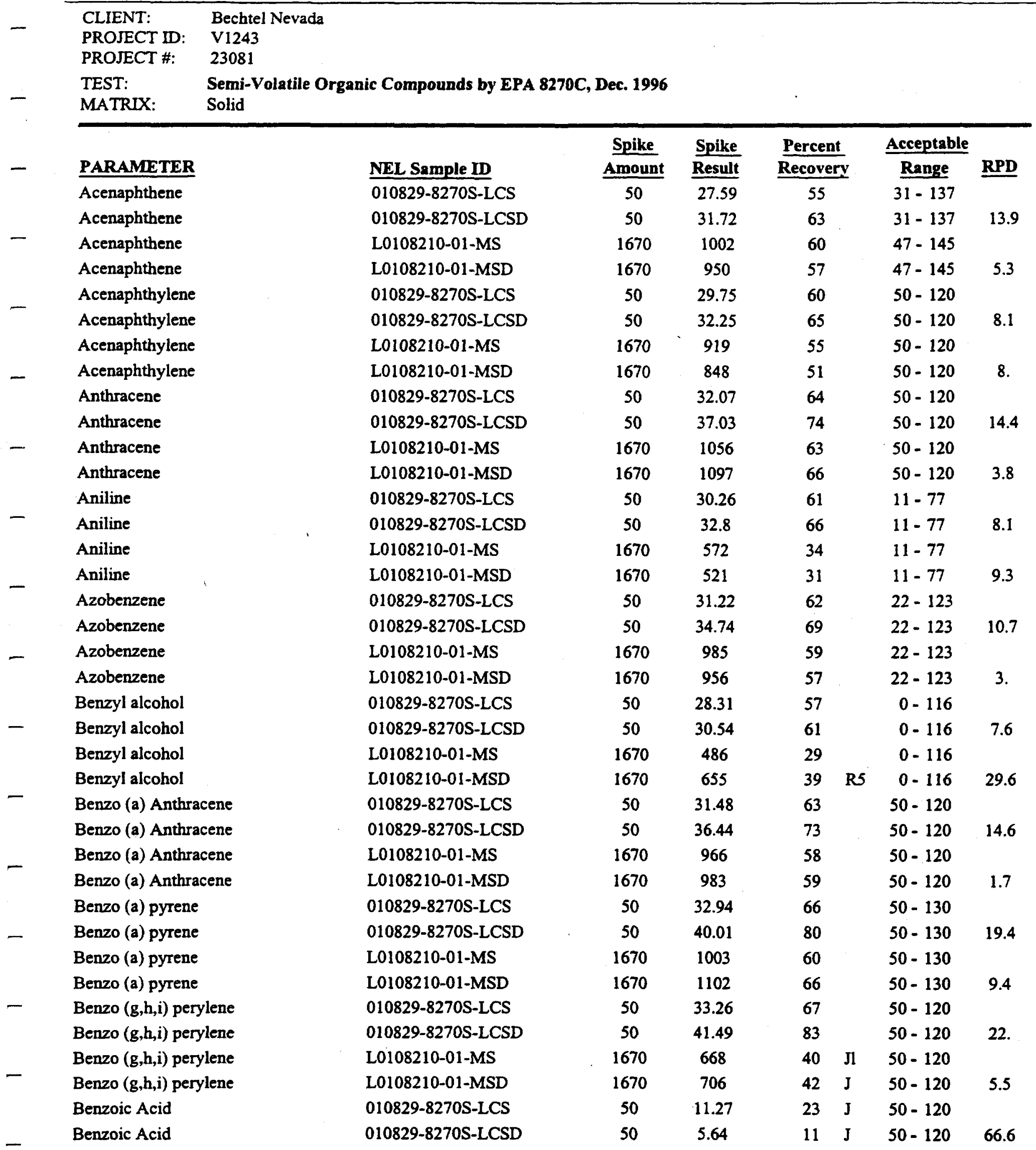

ND - Not Detected

This report shall not be reproduced except in full, without the written approval of the laboratory. 


$\begin{array}{ll}\text { CLIENT: } & \text { Bechtel Nevada } \\ \text { PROJECT ID: } & \text { V1243 } \\ \text { PROJECT \#: } & 23081 \\ \text { TEST: } & \text { Semi-Volatile Organic Compounds by EPA 8270C, Dec. } 1996 \\ \text { MATRIX: } & \text { Solid }\end{array}$

\begin{tabular}{|c|c|c|c|c|c|c|c|}
\hline PARAMETER & NEL Sample ID & $\underline{\text { Spike }}$ & $\begin{array}{l}\text { Spike } \\
\text { Result }\end{array}$ & $\frac{\text { Percent }}{\text { Recovery }}$ & & $\frac{\text { Acceptable }}{\underline{\text { Range }}}$ & $\underline{\mathbf{R P D}}$ \\
\hline Benzoic Acid & L0108210-01-MS & 1670 & 614 & 37 & $J$ & $50-120$ & \\
\hline Benzoic Acid & L0108210-01-MSD & 1670 & 568 & 34 & $\mathrm{Jl}$ & $50-120$ & 7.8 \\
\hline 4-Bromophenyl phenyl ether & 010829-8270S-LCS & 50 & 30.29 & 61 & & $50-120$ & \\
\hline 4-Bromophenyl phenyl ether & 010829-8270S-LCSD & 50 & 34.7 & 69 & & $50-120$ & 13.6 \\
\hline 4-Bromophenyl phenyl ether & L0108210-01-MS & 1670 & 1005 & 60 & & $50-120$ & \\
\hline 4-Bromophenyl phenyl ether & L0108210-01-MSD & 1670 & 992 & 59 & & $50-120$ & 1.3 \\
\hline Butyl benzyl phthalate & 010829-8270S-LCS & 50 & 34.02 & 68 & & $50-120$ & \\
\hline Butyl benzyl phthalate & 010829-8270S-LCSD & 50 & 38.82 & 78 & & $50-120$ & 13.2 \\
\hline Carbazole & 010829-8270S-LCS & 50 & 31.41 & 63 & & $50-120$ & \\
\hline Carbazole & 010829-8270S-LCSD & 50 & 35.13 & 70 & & $50-120$ & 11.2 \\
\hline Carbazole & L0108210-01-MS & 1670 & 1062 & 64 & & $50-120$ & \\
\hline Carbazole & L0108210-01-MSD & 1670 & 1084 & 65 & & $50-120$ & 2.1 \\
\hline 4-Chloroaniline & 010829-8270S-LCS & 50 & 25.69 & 51 & & $1-68$ & \\
\hline 4-Chloroaniline & 010829-8270S-LCSD & 50 & 26.03 & 52 & & $1-68$ & 1.3 \\
\hline Chrysene & 010829-8270S-LCS & 50 & 29.96 & 60 & & $50-120$ & \\
\hline Chrysene & 010829-8270S-LCSD & 50 & 36.9 & 74 & & $50-120$ & 20.8 \\
\hline Chrysene & L0108210-01-MS & 1670 & 1001 & 60 & & $50-120$ & \\
\hline Chrysene & L0108210-01-MSD & 1670 & 1028 & 62 & & $50-120$ & 2.7 \\
\hline bis (2-Chloroethoxy) methane & 010829-8270S-LCS & 50 & 26.73 & 53 & & $26-97$ & \\
\hline bis (2-Chloroethoxy) methane & 010829-8270S-LCSD & 50 & 30.19 & 60 & & $26-97$ & 12.2 \\
\hline bis (2-Chloroethoxy) methane & L0108210-01-MS & 1670 & 842 & 50 & & $26-97$ & \\
\hline bis (2-Chloroethoxy) methane & L0108210-01-MSD & 1670 & 789 & 47 & & $26-97$ & 6.5 \\
\hline bis (2-Chloroethyl) ether & 010829-8270S-LCS & 50 & 23.81 & 48 & & $15-107$ & \\
\hline bis (2-Chloroethyl) ether & 010829-8270S-LCSD & 50 & 28.51 & 57 & & $15-107$ & 18. \\
\hline bis (2-Chloroethyl) ether & L0108210-01-MS & 1670 & 891 & 53 & & $15-107$ & \\
\hline bis (2-Chloroethyl) ether & L0108210-01-MSD & 1670 & 749 & 45 & & $15-107$ & 17.3 \\
\hline bis (2-chloroisopropyl) ether & 010829-8270S-LCS & 50 & 27.01 & 54 & & $50-120$ & \\
\hline bis (2-chloroisopropyl) ether & 010829-8270S-LCSD & 50 & 30.02 & 60 & & $50-120$ & 10.6 \\
\hline bis (2-chloroisopropyl) ether & L0108210-01-MS & 1670 & 583.21 & 35 & $\mathrm{~J} 1$ & $50-120$ & \\
\hline bis (2-chloroisopropyl) ether & L0108210-01-MSD & 1670 & 489 & 29 & $J 1$ & $50-120$ & 17.6 \\
\hline 2-Chloronaphthalene & 010829-8270S-LCS & 50 & 28.98 & 58 & & $50-120$ & \\
\hline 2-Chloronaphthalene & 010829-8270S-LCSD & 50 & 30.75 & 62 & & $50-120$ & 5.9 \\
\hline 2-Chloronaphthalene & L0108210-01-MS & 1670 & 913 & 55 & & $50-120$ & \\
\hline 2-Chloronaphthalene & L0108210-01-MSD & 1670 & 861 & 52 & & $50-120$ & 5.9 \\
\hline 2-Chlorophenol & 010829-8270S-LCS & 50 & 26.44 & 53 & & $25-102$ & \\
\hline 2-Chlorophenol & 010829-8270S-LCSD & 50 & 29.22 & 58 & & $25-102$ & 10. \\
\hline 2-Chlorophenol & L0108210-01-MS & 1670 & 854 & 51 & & $25-102$ & \\
\hline 2-Chlorophenol & L0108210-01-MSD & 1670 & 766 & 46 & & $25-102$ & 10.9 \\
\hline
\end{tabular}

ND - Not Detected

This report shall not be reproduced except in full, without the written approval of the laboratory. 
NEL LABORATORIES

CLIENT: Bechtel Nevada

PROJECT ID: V1243

PROJECT \#: $\quad 23081$

TEST: $\quad$ Semj-Volatile Organic Compounds by EPA 8270C, Dec. 1996

MATRIX: $\quad$ Solid

\begin{tabular}{|c|c|c|c|c|c|c|c|}
\hline PARAMETER & NEL Sample ID & $\frac{\text { Spike }}{\text { Amount }}$ & $\underline{\text { Spike }}$ & $\begin{array}{l}\text { Percent } \\
\text { Recover }\end{array}$ & & $\frac{\text { Acceptable }}{\text { Range }}$ & $\underline{\text { RPD }}$ \\
\hline 4-Chloro-3-methyl phenol & 010829-8270S-LCS & 50 & 28.82 & 58 & & $26-103$ & \\
\hline 4-Chloro-3-methyl phenol & 010829-8270S-LCSD & 50 & 33.97 & 68 & & $26-103$ & 16.4 \\
\hline 4-Chloro-3-methyl phenol & L0108210-01-MS & 1670 & 833 & 50 & & $26-103$ & \\
\hline 4-Chloro-3-methyl phenol & L0108210-01-MSD & 1670 & 853 & 51 & & $26-103$ & 2.4 \\
\hline 4-Chlorophenyl phenyl ether & 010829-8270S-LCS & 50 & 28.62 & 57 & & $50-120$ & \\
\hline 4-Chlorophenyl phenyl ether & 010829-8270S-LCSD & 50 & 33.37 & 67 & & $50-120$ & 15.3 \\
\hline 4-Chlorophenyl phenyl ether & L0108210-01-MS & 1670 & 723 & 43 & Л & $50-120$ & \\
\hline 4-Chlorophenyl phenyl ether & L0108210-01-MSD & 1670 & 747 & 45 & Л & $50-120$ & 3.3 \\
\hline Dibenz $(a, h)$ anthracene & 010829-8270S-LCS & 50 & 32.1 & 64 & & $50-120$ & \\
\hline Dibenz $(a, h)$ anthracene & 010829-8270S-LCSD & 50 & 40 & 80 & & $50-120$ & 21.9 \\
\hline Dibenzofuran & 010829-8270S-LCS & 50 & 28.04 & 56 & & $50-120$ & \\
\hline Dibenzofuran & 010829-8270S-LCSD & 50 & 32.12 & 64 & & $50-120$ & 13.6 \\
\hline Dibenzofuran & L0108210-01-MS & 1670 & 845 & 51 & & $50-120$ & \\
\hline Dibenzofuran & L0108210-01-MSD & 1670 & 866 & 52 & & $50-120$ & 2.5 \\
\hline 1,2-Dichlorobenzene (o-DCB) & 010829-8270S-LCS & 50 & 24.42 & 49 & & $42-93$ & \\
\hline 1,2-Dichlorobenzene (o-DCB) & 010829-8270S-LCSD & 50 & 26.89 & 54 & & $42-93$ & 9.6 \\
\hline 1,2-Dichlorobenzene (o-DCB) & L0108210-01-MS & 1670 & 850 & 51 & & $42-93$ & \\
\hline 1,2-Dichlorobenzene (o-DCB) & L0108210-01-MSD & 1670 & 727 & 44 & & $42-93$ & 15.6 \\
\hline 1,3-Dichlorobenzene (m-DCB) & 010829-8270S-LCS & 50 & 22.52 & 45 & & $41-89$ & \\
\hline 1,3-Dichlorobenzene (m-DCB) & 010829-8270S-LCSD & 50 & 26.34 & 53 & & $41-89$ & 15.6 \\
\hline 1,3-Dichlorobenzene (m-DCB) & L0108210-01-MS & 1670 & 817 & 49 & & $41-89$ & \\
\hline 1,3-Dichlorobenzene (m-DCB) & L0108210-01-MSD & 1670 & 684 & 41 & Л & $41-89$ & 17.7 \\
\hline 1,4-Dichlorobenzene ( $\mathrm{p}-\mathrm{DCB}$ ) & 010829-8270S-LCS & 50 & 23.72 & 47 & & $42-93$ & \\
\hline 1,4-Dichlorobenzene (p-DCB) & 010829-8270S-LCSD & 50 & 27.62 & 55 & & $42-93$ & 15.2 \\
\hline 1,4-Dichlorobenzene (p-DCB) & L0108210-01-MS & 1670 & 822 & 49 & & $42-93$ & \\
\hline 1,4-Dichlorobenzene (p-DCB) & L0108210-01-MSD & 1670 & 692 & 41 & л & $42-93$ & 17.2 \\
\hline 3,3'-Dichlorobenzidine & 010829-8270S-LCS & 50 & 27.56 & 55 & & $11-85$ & \\
\hline 3,3'-Dichlorobenzidine & 010829-8270S-LCSD & 50 & 32.03 & 64 & & $11-85$ & 15. \\
\hline 3,3'-Dichlorobenzidine & L0108210-01-MS & 1670 & 647 & 39 & & $11-85$ & \\
\hline 3,3'-Dichlorobenzidine & L0108210-01-MSD & 1670 & 698 & 42 & & $11-85$ & 7.6 \\
\hline 2,4-Dichlorophenol & 010829-8270S-LCS & 50 & 27.34 & 55 & & $50-120$ & \\
\hline 2,4-Dichlorophenol & 010829-8270S-LCSD & 50 & 30.3 & 61 & & $50-120$ & 10.3 \\
\hline 2,4-Dichlorophenol & L0108210-01-MS & 1670 & 856 & 51 & & $50-120$ & \\
\hline 2,4-Dichlorophenol & L0108210-01-MSD & 1670 & 839 & 50 & & $50-120$ & 2. \\
\hline 2,4-Dimethylphenol & 010829-8270S-LCS & 50 & 25.84 & 52 & & $50-120$ & \\
\hline 2,4-Dimethylphenol & 010829-8270S-LCSD & 50 & 29.43 & 59 & & $50-120$ & 13. \\
\hline 2,4-Dimethylphenol & L0108210-01-MS & 1670 & 454 & 27 & Л & $50-120$ & \\
\hline 2,4-Dimethylphenol & L0108210-01-MSD & 1670 & 428 & 26 & Л & $50-120$ & 5.9 \\
\hline
\end{tabular}

ND - Not Detected

This report shall not be reproduced except in full, without the written approval of the laboratory. 


$\begin{array}{ll}\text { CLIENT: } & \text { Bechtel Nevada } \\ \text { PROJECT ID: } & \text { V1243 } \\ \text { PROJECT \#: } & 23081 \\ \text { TEST: } & \text { Semi-Volatile Organic Compounds by EPA 8270C, Dec. } 1996 \\ \text { MATRIX: } & \text { Solid }\end{array}$

\begin{tabular}{|c|c|c|c|c|c|c|}
\hline PARAMETER & NEL Sample ID & $\begin{array}{c}\text { Spike } \\
\text { Amount }\end{array}$ & $\begin{array}{l}\text { Spike } \\
\text { Result }\end{array}$ & $\frac{\text { Percent }}{\text { Recovery }}$ & $\frac{\text { Acceptable }}{\text { Range }}$ & $\underline{\mathbf{R P D}}$ \\
\hline 4,6-Dinitro-2-methyl phenol & 010829-8270S-LCS & 50 & 28.97 & 58 & $20-120$ & \\
\hline 4,6-Dinitro-2-methyl phenol & 010829-8270S-LCSD & 50 & 32.77 & 66 & $20-120$ & 12.3 \\
\hline 4,6-Dinitro-2-methyl phenol & L0108210-01-MS & 1670 & 923 & 55 & $20-120$ & \\
\hline 4,6-Dinitro-2-methyl phenol & L0108210-01-MSD & 1670 & 993 & 59 & $20-120$ & 7.3 \\
\hline 2,4-Dinitrophenol & 010829-8270S-LCS & 50 & 15.58 & 31 & $20-120$ & \\
\hline 2,4-Dinitrophenol & 010829-8270S-LCSD & 50 & 19.11 & 38 & $20-120$ & 20.4 \\
\hline 2,4-Dinitrophenol & L0108210-01-MS & 1670 & 753 & 45 & $20-120$ & \\
\hline 2,4-Dinitrophenol & L0108210-01-MSD & 1670 & 801 & 48 & $20-120$ & 6.2 \\
\hline 2,4-Dinitrotoluene (DNT) & 010829-8270S-LCS & 50 & 30.27 & 61 & $50-111$ & \\
\hline 2,4-Dinitrotoluene (DNT) & 010829-8270S-LCSD & 50 & 34.39 & 69 & $50-111$ & 12.7 \\
\hline 2,4-Dinitrotoluene (DNT) & L0108210-01-MS & 1670 & 954 & 57 & $50-111$ & \\
\hline 2,4-Dinitrotoluene (DNT) & L0108210-01-MSD & 1670 & 1023 & 61 & $50-111$ & 7. \\
\hline 2,6-Dinitrotoluene (DNT) & $010829-8270 S-L C S$ & 50 & 32.04 & 64 & $50-120$ & \\
\hline 2,6-Dinitrotoluene (DNT) & 010829-8270S-LCSD & 50 & 36.77 & 74 & $50-120$ & 13.7 \\
\hline 2,6-Dinitrotoluene (DNT) & L0108210-01-MS & 1670 & 906 & 54 & $50-120$ & \\
\hline 2,6-Dinitrotoluene (DNT) & L0108210-01-MSD & 1670 & 952 & 57 & $50-120$ & 5. \\
\hline Diethyl Phthalate & 010829-8270S-LCS & 50 & 29.21 & 58 & $50-120$ & \\
\hline Diethyl Phthalate & 010829-8270S-LCSD & 50 & 37.34 & 75 & $50-120$ & 24.4 \\
\hline Dimethyl phthalate & 010829-8270S-LCS & 50 & 30.48 & 61 & $50-120$ & \\
\hline Dimethyl phthalate & 010829-8270S-LCSD & 50 & 36.03 & 72 & $50-120$ & 16.7 \\
\hline Di-n-butyl phthalate & 010829-8270S-LCS & 50 & 33.17 & 66 & $50-120$ & \\
\hline Di-n-butyl phthalate & 010829-8270S-LCSD & 50 & 38.47 & 77 & $50-120$ & 14.8 \\
\hline Di-n-butyl phthalate & L0108210-01-MS & 1670 & 1127 & 67 & $50-120$ & \\
\hline Di-n-butyl phthalate & L0108210-01-MSD & 1670 & 1154 & 69 & $50-120$ & 2.4 \\
\hline Di-n-octyl phthalate & 010829-8270S-LCS & 50 & 34.59 & 69 & $50-120$ & \\
\hline Di-n-octyl phthalate & 010829-8270S-LCSD & 50 & 40.92 & 82 & $50-120$ & 16.8 \\
\hline Di-n-octyl phthalate & L0108210-01-MS & 1670 & 1061 & 64 & $50-120$ & \\
\hline Di-n-octyl phthalate & L0108210-01-MSD & 1670 & 1118 & 67 & $50-120$ & 5.2 \\
\hline bis (2-Ethylhexyl)phthalate & 010829-8270S-LCS & 50 & 31.6 & 63 & $50-120$ & \\
\hline bis (2-Ethylhexyl)phthalate & 010829-8270S-LCSD & 50 & 37.65 & 75 & $50-120$ & 17.5 \\
\hline bis (2-Ethylhexyl)phthalate & L0108210-01-MS & 1670 & 1197 & 72 & $50-120$ & \\
\hline bis (2-Ethylhexyl)phthalate & L0108210-01-MSD & 1670 & 1265 & 76 & $50-120$ & 5.5 \\
\hline Flouranthene & 010829-8270S-LCS & 50 & 32.86 & 66 & $50-120$ & \\
\hline Flouranthene & 010829-8270S-LCSD & 50 & 38.55 & 77 & $50-120$ & 15.9 \\
\hline Flourene & 010829-8270S-LCS & 50 & 29.29 & 59 & $50-120$ & \\
\hline Flourene & 010829-8270S-LCSD & 50 & 34.62 & 69 & $50-120$ & 16.7 \\
\hline Hexachlorobenzene & 010829-8270S-LCS & 50 & 31.56 & 63 & $50-120$ & \\
\hline Hexachlorobenzene & 010829-8270S-LCSD & 50 & 33.36 & 67 & $50-120$ & 5.5 \\
\hline
\end{tabular}

ND - Not Detected

This report shall not be reproduced except in full, without the written approval of the laboratory. 


$\begin{array}{ll}\text { CLIENT: } & \text { Bechtel Nevada } \\ \text { PROJECT D: } & \text { V1243 } \\ \text { PROJECT \#: } & 23081 \\ \text { TEST: } & \text { Semi-Volatile Organic Compounds by EPA 8270C, Dec. } 1996 \\ \text { MATRIX: } & \text { Solid }\end{array}$

\begin{tabular}{|c|c|c|c|c|c|c|c|}
\hline PARAMETER & NEL Sample ID & Spike & $\underline{\text { Spike }}$ & $\underline{\text { Percent }}$ & & $\frac{\text { Acceptable }}{\underline{\text { Range }}}$ & $\underline{\mathbf{R P D}}$ \\
\hline Hexachlorobenzene & L0108210-01-MS & 1670 & 1022 & 61 & & $50-120$ & \\
\hline Hexachlorobenzene & L0108210-01-MSD & 1670 & 1067 & 64 & & $50-120$ & 4.3 \\
\hline Hexachlorobutadiene & 010829-8270S-LCS & 50 & 24.82 & 50 & & $30-120$ & \\
\hline Hexachlorobutadiene & 010829-8270S-LCSD & 50 & 26.43 & 53 & & $30-120$ & 6.3 \\
\hline Hexachlorobutadiene & L0108210-01-MS & 1670 & 584 & 35 & & $30-120$ & \\
\hline Hexachlorobutadiene & L0108210-01-MSD & 1670 & 562 & 34 & & $30-120$ & 3.8 \\
\hline Hexachlorocyclopentadiene & 010829-8270S-LCS & 50 & 22.66 & 45 & & $20-120$ & \\
\hline Hexachlorocyclopentadiene & 010829-8270S-LCSD & 50 & 25.49 & 51 & & $20-120$ & 11.8 \\
\hline Hexachlorocyclopentadiene & L0108210-01-MS & 1670 & 421 & 25 & & $20-120$ & \\
\hline Hexachlorocyclopentadiene & L0108210-01-MSD & 1670 & 328 & 20 & $\mathrm{Jl}$ & $20-120$ & 24.8 \\
\hline Hexachloroethane & 010829-8270S-LCS & 50 & 21.49 & 43 & & $30-120$ & \\
\hline Hexachloroethane & 010829-8270S-LCSD & 50 & 27.1 & 54 & & $30-120$ & 23.1 \\
\hline Hexachloroethane & L0108210-01-MS & 1670 & 709 & 42 & & $30-120$ & \\
\hline Hexachloroethane & L0108210-01-MSD & 1670 & 549 & 33 & R5 & $30-120$ & 25.4 \\
\hline Indeno $(1,2,3-c, d)$ pyrene & 010829-8270S-LCS & 50 & 30.82 & 62 & & $50-120$ & \\
\hline Indeno $(1,2,3-c, d)$ pyrene & 010829-8270S-LCSD & 50 & 39.56 & 79 & & $50-120$ & 24.8 \\
\hline Indeno $(1,2,3-c, d)$ pyrene & L0108210-01-MS & 1670 & 787 & 47 & 几 & $50-120$ & \\
\hline Indeno $(1,2,3-c, d)$ pyrene & L0108210-01-MSD & 1670 & 843 & 50 & & $50-120$ & 6.9 \\
\hline Isophorone & 010829-8270S-LCS & 50 & 27.17 & 54 & & $24-99$ & \\
\hline Isophorone & 010829-8270S-LCSD & 50 & 29.34 & 59 & & $24-99$ & 7.7 \\
\hline Isophorone & L0108210-01-MS & 1670 & 761 & 46 & & $24-99$ & \\
\hline Isophorone & L0108210-01-MSD & 1670 & 699 & 42 & & $24-99$ & 8.5 \\
\hline 2-Methylnaphthalene & 010829-8270S-LCS & 50 & 25.78 & 52 & & $50-120$ & \\
\hline 2-Methyinaphthalene & 010829-8270S-LCSD & 50 & 28.29 & 57 & & $50-120$ & 9.3 \\
\hline 2-Methylnaphthalene & L0108210-01-MS & 1670 & 842 & 50 & & $50-120$ & \\
\hline 2-Methylnaphthalene & L0108210-01-MSD & 1670 & 814 & 49 & $\pi$ & $50-120$ & 3.4 \\
\hline 2-Methylphenol & 010829-8270S-LCS & 50 & 26.65 & 53 & & $14-108$ & \\
\hline 2-Methylphenol & 010829-8270S-LCSD & 50 & 28.21 & 56 & & $14-108$ & 5.7 \\
\hline 2-Methylphenol & L0108210-01-MS & 1670 & 870 & 52 & & $14-108$ & \\
\hline 2-Methylphenol & L0108210-01-MSD & 1670 & 757 & 45 & & $14-104$ & 13.9 \\
\hline Naphthalene & 010829-8270S-LCS & 50 & 27.76 & 56 & & $50-120$ & \\
\hline Naphthalene & 010829-8270S-LCSD & 50 & 29.17 & 58 & & $50-120$ & 5. \\
\hline Naphthalene & L0108210-01-MS & 1670 & 792 & 47 & $\boldsymbol{\Omega}$ & $50-120$ & \\
\hline Naphthalene & L0108210-01-MSD & 1670 & 757 & 45 & $\boldsymbol{\Omega}$ & $50-120$ & 4.5 \\
\hline 2-Nitroaniline & 010829-8270S-LCS & 50 & 28.99 & 58 & & $30-120$ & \\
\hline 2-Nitroaniline & 010829-8270S-LCSD & 50 & 34.88 & 70 & & $30-120$ & 18.4 \\
\hline 2-Nitroaniline & L0108210-01-MS & 1670 & 740 & 44 & & $30-120$ & \\
\hline 2-Nitroaniline & L0108210-01-MSD & 1670 & 771 & 46 & & $30-120$ & 4.1 \\
\hline \multicolumn{8}{|l|}{ ND - Not Detected } \\
\hline \multicolumn{8}{|c|}{$\begin{array}{l}\text { This report shall not be reproduced except in full, without the written approval of the laboratory. } \\
31\end{array}$} \\
\hline
\end{tabular}




$\begin{array}{ll}\text { CLIENT: } & \text { Bechtel Nevada } \\ \text { PROJECT ID: } & \text { V1243 } \\ \text { PROJECT \#: } & 23081 \\ \text { TEST: } & \text { Semi-Volatile Organic Compounds by EPA 8270C, Dec. } 1996 \\ \text { MATRIX: } & \text { Solid }\end{array}$

\begin{tabular}{|c|c|c|c|c|c|c|c|}
\hline PARAMETER & NEL Sample ID & $\begin{array}{c}\text { Spike } \\
\text { Amount }\end{array}$ & $\frac{\text { Spike }}{\underline{\text { Result }}}$ & $\frac{\text { Percent }}{\text { Recovery }}$ & & $\frac{\text { Acceptable }}{\text { Range }}$ & $\underline{\mathbf{R P D}}$ \\
\hline 3-Nitroaniline & 010829-8270S-LCS & 50 & 29.05 & 58 & & $30-120$ & \\
\hline 3-Nitroaniline & 010829-8270S-LCSD & 50 & 35.72 & 71 & & $30-120$ & 20.6 \\
\hline 3-Nitroaniline & L0108210-01-MS & 1670 & 713 & 43 & & $30-120$ & \\
\hline 3-Nitroaniline & L0108210-01-MSD & 1670 & 823 & 49 & & $30-120$ & 14.3 \\
\hline 4-Nitroaniline & 010829-8270S-LCS & 50 & 29.62 & 59 & & $30-120$ & \\
\hline 4-Nitroaniline & 010829-8270S-LCSD & 50 & 34.24 & 68 & & $30-120$ & 14.5 \\
\hline 4-Nitroaniline & L0108210-01-MS & 1670 & 818 & 49 & & $30-120$ & \\
\hline 4-Nitroaniline & L0108210-01-MSD & 1670 & 877 & 53 & & $30-120$ & 7. \\
\hline 2-Nitrophenol & 010829-8270S-LCS & 50 & 25.62 & 51 & & $42-86$ & \\
\hline 2-Nitrophenol & 010829-8270S-LCSD & 50 & 27.94 & 56 & & $42-86$ & 8.7 \\
\hline 2-Nitrophenol & L0108210-01-MS & 1670 & 780 & 47 & & $42-86$ & \\
\hline 2-Nitrophenol & L0108210-01-MSD & 1670 & 727 & 44 & & $42-86$ & 7. \\
\hline 4-Nitrophenol & 010829-8270S-LCS & 50 & 27.06 & 54 & & $22-151$ & \\
\hline 4-Nitrophenol & $010829-8270 S-L C S D$ & 50 & 36.75 & 74 & R5 & $22-151$ & 30.4 \\
\hline 4-Nitrophenol & L0108210-01-MS & 1670 & 780 & 47 & & $22-151$ & \\
\hline 4-Nitrophenol & L0108210-01-MSD & 1670 & 866 & 52 & & $22-151$ & 10.4 \\
\hline Nitrobenzene & 010829-8270S-LCS & 50 & 26.95 & 54 & & $23-103$ & \\
\hline Nitrobenzene & 010829-8270S-LCSD & 50 & 30.33 & 61 & & $23-103$ & 11.8 \\
\hline Nitrobenzene & L0108210-01-MS & 1670 & 723 & 43 & & $23-103$ & \\
\hline Nitrobenzene & L0108210-01-MSD & 1670 & 626 & 37 & & $23-103$ & 14.4 \\
\hline N-Nitrosodimethylamine & 010829-8270S-LCS & 50 & 28.38 & 57 & & $9-97$ & \\
\hline N-Nitrosodimethylamine & 010829-8270S-LCSD & 50 & 29.34 & 59 & & $9-97$ & 3.3 \\
\hline N-Nitrosodimethylamine & L0108210-01-MS & 1670 & 500.6 & 30 & & $9-97$ & \\
\hline N-Nitrosodimethylamine & L0108210-01-MSD & 1670 & 378 & 23 & R5 & $9-97$ & 27.9 \\
\hline N-Nitrosodiphenylamine & 010829-8270S-LCS & 50 & 31.88 & 64 & & $50-120$ & \\
\hline N-Nitrosodiphenylamine & 010829-8270S-LCSD & 50 & 37.39 & 75 & & $50-120$ & 15.9 \\
\hline N-Nitrosodiphenylamine & L0108210-01-MS & 1670 & 1113 & 67 & & $50-120$ & \\
\hline N-Nitrosodiphenylamine & L0108210-01-MSD & 1670 & 1111 & 67 & & $50-120$ & 0.2 \\
\hline N-Nitrosodi-n-propylamine & 010829-8270S-LCS & 50 & 25.88 & 52 & & $41-126$ & \\
\hline N-Nitrosodi-n-propylamine & 010829-8270S-LCSD & 50 & 31.33 & 63 & & $41-126$ & 19.1 \\
\hline N-Nitrosodi-n-propylamine & L0108210-01-MS & 1670 & 854 & 51 & & $41-126$ & \\
\hline N-Nitrosodi-n-propylamine & L0108210-01-MSD & 1670 & 787 & 47 & & $41-126$ & 8.2 \\
\hline Pentachlorophenol & 010829-8270S-LCS & 50 & 24.6 & 49 & & $17-109$ & \\
\hline Pentachlorophenol & 010829-8270S-LCSD & 50 & 27.93 & 56 & & $17-109$ & 12.7 \\
\hline Pentachlorophenol & L0108210-01-MS & 1670 & 735 & 44 & & $17-109$ & \\
\hline Pentachlorophenol & L0108210-01-MSD & 1670 & 886 & 53 & & $17-109$ & 18.6 \\
\hline Phenanthrene & 010829-8270S-LCS & 50 & 30.6 & 61 & & $50-120$ & \\
\hline Phenanthrene & 010829-8270S-LCSD & 50 & 34.14 & 68 & & $50-120$ & 10.9 \\
\hline
\end{tabular}

ND - Not Detected

This report shall not be reproduced except in full, without the written approval of the laboratory. 


\section{CLIENT: Bechtel Nevada}

PROJECT ID: V1243

PROJECT \#: $\quad 23081$

TEST: Semi-Volatile Organic Compounds by EPA 8270C, Dec. 1996

MATRIX: $\quad$ Solid

- MATRIX: Solid

- PARAMETER

Phenanthrene

Phenanthrene

- Phenol

Phenol

Phenol

Phenol

Pyrene

Pyrene

Pyrene

Pyrene

Pyridine

Pyridine

Pyridine

Pyridine

1,2,4-Trichlorobenzene

1,2,4-Trichlorobenzene

1,2,4-Trichlorobenzene

1,2,4-Trichlorobenzene

2,4,5-Trichloropheno]

2,4,5-Trichloropheno]

2,4,5-Trichlorophenol

2,4,5-Trichlorophenol

2,4,6-Trichlorophenol

2,4,6-Trichlorophenol

2,4,6-Trichlorophenol

2,4,6-Trichlorophenol
NEL Sample ID

L0108210-01-MS

L0108210-01-MSD

010829-8270S-LCS

010829-8270S-LCSD

L0108210-01-MS

L0108210-01-MSD

010829-8270S-LCS

010829-8270S-LCSD

L0108210-01-MS

L0108210-01-MSD

010829-8270S-LCS

010829-8270S-LCSD

L0108210-01-MS

L0108210-01-MSD

010829-8270S-LCS

010829-8270S-LCSD

L0108210-01-MS

L0108210-01-MSD

010829-8270S-LCS

010829-8270S-LCSD

L0108210-01-MS

L0108210-01-MSD

010829-8270S-LCS

010829-8270S-LCSD

L0108210-01-MS

L0108210-01-MSD

\begin{tabular}{|c|c|}
\hline$\frac{\text { Spike }}{\text { Amount }}$ & $\begin{array}{l}\text { Spike } \\
\text { Result }\end{array}$ \\
\hline 1670 & 1069 \\
\hline 1670 & 1098 \\
\hline 50 & 27.66 \\
\hline 50 & 32.09 \\
\hline 1670 & 957 \\
\hline 1670 & 838 \\
\hline 50 & 30.51 \\
\hline 50 & 35.56 \\
\hline 1670 & 923 \\
\hline 1670 & 944 \\
\hline 50 & 20.7 \\
\hline 50 & 24.79 \\
\hline 1670 & 545 \\
\hline 1670 & 387 \\
\hline 50 & 26.26 \\
\hline 50 & 27.94 \\
\hline 1670 & 765 \\
\hline 1670 & 689 \\
\hline 50 & 30.11 \\
\hline 50 & 36.4 \\
\hline 1670 & 799 \\
\hline 1670 & 818 \\
\hline 50 & 29.31 \\
\hline 50 & 32.01 \\
\hline 1670 & 820 \\
\hline 1670 & 759 \\
\hline
\end{tabular}

$\frac{\text { Percent }}{\text { Recover }}$

64

66

55

64

57

50

61

71

55

57

41

50

33

23

53

56

46

41

60

60
73

48

48

49 J

59

64

$49 \mathrm{~J}$

$45 \mathrm{~J}$
Acceptable Range RPD

$50-120$

$50-120$

2.7

$52-113$

$52-113$

14.8

$52-113$

$52-113$

13.3

$50-120$

$50-120$

15.3

$50-120$

$50-120$

$4-71$

$4-71$

18.

$4-71$

R5 $4-71$

$38-107$

$38-107$

$38-107$

$38-107$

$50-120$

$50-120$

18.9

33.9

6.2

10.5

$50-120$

$50-120$

2.4

$50-120$

$50-120$

$50-120$

$50-120$

7.7

ND - Not Detected

This report shall not be reproduced except in full, without the written approval of the laboratory. 
NEL LABORATORIES

\section{CLIENT: $\quad$ Bechtel Nevada}

PROJECT ID: V1243

PROJECT \#: 23081

TEST: $\quad$ Total Extractable Petroleum Hydrocarbons Fuel Finger Print by EPA Method 8015M, July 1992

MATRIX: $\quad$ Solid

\begin{tabular}{|c|c|c|c|c|c|c|}
\hline PARAMETER & NEL Sample ID & $\begin{array}{c}\text { Spike } \\
\text { Amount }\end{array}$ & $\frac{\text { Spike }}{\text { Result }}$ & Percent & $\frac{\text { Acceptable }}{\text { Range }}$ & $\underline{\mathbf{R P D}}$ \\
\hline Diesel Range (C12-C22) & 010904TPHS-1-LCS & 166.7 & 99 & 59 & $53-91$ & \\
\hline Diesel Range (C12-C22) & 010904TPHS-1-LCSD & 166.7 & 108 & 65 & $53-91$ & 8.7 \\
\hline Total & 010904TPHS-1-LCS & 166.7 & 99 & 59 & $53-91$ & \\
\hline Total & 010904TPHS-1-LCSD & 166.7 & 108 & 65 & $53-91$ & 8.7 \\
\hline
\end{tabular}

ND - Not Detected

This report shall not be reproduced except in full, without the written approval of the laboratory. 


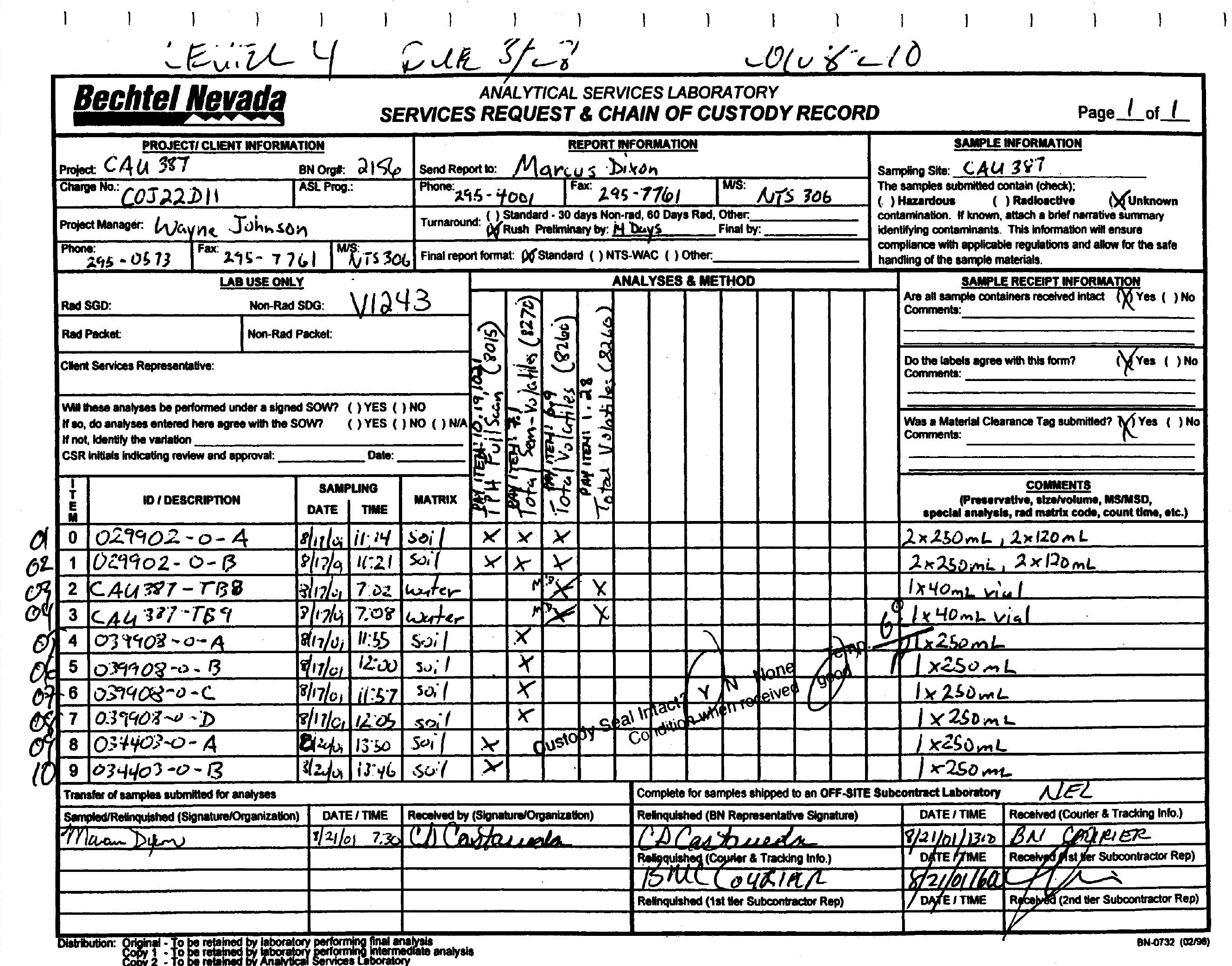


CLIENT:

Bechtel Nevade

P.O. Box 98521, M/S NTS273

Las Vegas, NV 89193-8521

ATIN:

Ted Redding

PROJECT NAME: V1282

NEL ORDER ID: 20109246

PROIECT NUMBER: 23081

Attached are the analytionl results for saroles in aupport of the above referenced project.

Samples submined for this project were not sempled by NEI Labcistocies. Samples were received by NEC in good condition, under chain of custody on 9/25/01.

Should you have any questions or comments, please feal free to coneset our Client Services deparment as (702) 657-1010.

Some QA results have been Aagged as follows:

J - This concentration should be considered an estimate due bbordtory control sample failure.

ת - The batch MS and/or MSD were outside accepennec limuts. The batch LCS was acceptuble.

CERTIFICATIONS:

\begin{tabular}{llll}
\hline & Reno & Las Vegas & S. Califomin \\
Arizana & AZOS20 & AZOS18 & AZ0605 \\
Califormia & 1707 & 2002 & 2264 \\
US Army Corps & Certified & Certified & \\
of Engincers. & & &
\end{tabular}

İabo Monana Nevada

I.A.C.S.D.
Reno Las Vegas S. Califormin Certified Certified Certified Certified NV033 NV052 


\begin{tabular}{|c|c|c|c|c|c|c|c|}
\hline $\begin{array}{l}\text { CLIENT: } \\
\text { PROJECT ID: } \\
\text { PROJECT \#: }\end{array}$ & $\begin{array}{l}\text { Becherl Nevada } \\
\text { V1282 } \\
23081\end{array}$ & & & $\begin{array}{l}\text { CLIENT D: } \\
\text { DATE SAMPLED: } \\
\text { NEI SAMPLE DD: }\end{array}$ & $\begin{array}{l}039908-0-A \\
9 / 24 / 01 \\
{[0109246-01}\end{array}$ & & \\
\hline $\begin{array}{l}\text { TEST: } \\
\text { MEIHOD: } \\
\text { MAIRDX: } \\
\text { DIUTION: }\end{array}$ & $\begin{array}{l}\text { Semi-Volatile O } \\
\text { EPA } 8270 \\
\text { Solid } \\
1 \\
\end{array}$ & anic Com & ands by EP & $\begin{array}{l}\text { 270C, Dec. } 1996 \\
\text { EXIRACIED: } \\
\text { ANALYZED: } \\
\text { ANALYST: } \\
\end{array}$ & $\begin{array}{l}9 / 28 / 01 \\
10 / 8 / 01 \\
\text { SKV - Las Vega } \\
\end{array}$ & Is Division & \\
\hline PARAMETER & & $\begin{array}{l}\text { Result } \\
\text { Mo/kg }\end{array}$ & $\begin{array}{c}\text { Reporting } \\
\text { Limtt } \\
\end{array}$ & PARAMLIER & & $\begin{array}{l}\text { Result } \\
\text { Mg/kg }\end{array}$ & $\begin{array}{c}\text { Beportid } \\
\text { Limit } \\
\end{array}$ \\
\hline Acenuphchene & & ND & $330 . \mu g / k_{8}$ & Dinethyl phthalate & & ND & $330 . \mu \mathrm{g} / \mathrm{kg}$ \\
\hline Acenaphthylens & & ND & 330. netke & 4,6-Dinitro-2-methyl pher & thenol & ND & 330. $\mathrm{kg} / \mathrm{k}$ \\
\hline Anilide & & ND & 330. $\mu \mathrm{g} / \mathrm{kg}$ & 2,4-Dinitrotoluene (DNT) & VT) & ND & 330. $\mu \mathrm{g} / \mathrm{k}$ \\
\hline Anthracene & & ND & $330 . \mu \mathrm{B} / \mathrm{kg}$ & 26-Dinitrotoluene (DNT) & $\sqrt{T}$ & ND & 330. He/ks \\
\hline Azobenoreace & & ND & 330. $\mu 0 / \mathrm{ks}$ & 2,4-Dinitrophemol & & ND & 330. Hg/kg \\
\hline Beozo (a) anthreoce & & ND & 330. $\mathrm{HB}$ kg & Di-n-oetyl pbchaluce & & ND & 330. $\mathrm{ng} / \mathrm{kam}$ \\
\hline Benzo (b) Avorant & thene & ND & 330. ne/ks & Fhuoranthene & & ND & 330. $\mu \mathrm{g} / \mathrm{kg}$ \\
\hline Benzo (k) nuoranet & hene & ND & 330. $\mathrm{kg} / \mathrm{ks}$ & Fluorwe & & ND & 330. Me/kE \\
\hline Benzoic Acid & & ND & 330. $\mu 0 / \mathrm{kB}$ & Hexachlorobeneresce & & ND & 330. $\mu / \mathrm{kg}$ \\
\hline Bemzo (g,his peryl & & ND & 330. neteg & Hexneblorobunadiene & & ND & 330. $\mu \mathrm{g} / \mathrm{kg}$ \\
\hline Benzo (s) pyreace & & ND & 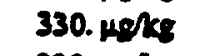 & Hexachlorocyelopentadie & diene & ND & 330. $\mu \mathrm{g} / \mathrm{kg}$ \\
\hline Benzyl alcohol & & ND & 330. $\mu g / \mathrm{kg}$ & Hexechloroechase & & ND & 330. ne/ks \\
\hline bis (2-Chloroethyl) & enter & ND & 330. waks & Indeno $(1,2,3-c, d)$ pyreace & & ND & 330. Me/k \\
\hline bis (2-Chioroechox) & y) methine & ND & 330. ke/k8 & Jeophoreme & & ND & 330. $\mathrm{He} / \mathrm{kg}$ \\
\hline bis (2-chloroisopro & pyl) ether & ND & 330. $\mu 8 / \mathrm{kg}$ & 2-Methylnaphthalewe & & ND & 330. $\mu \mathrm{e} / \mathrm{kg}$ \\
\hline bis (2-Ethylhexyl)p & ahthriate & ND & 330. मe/ks & 2-Methytphenol & & ND & 330. $\mathrm{H} / \mathrm{kg}$ \\
\hline Butyl benzyl phthal & & ND & 330. $\mu g / k g$ & 3,4-Methytphenol (irome & meric patr) & ND & 330. MC/ke \\
\hline 4-Bromopheayl phe & enyl ther & ND & 330. 198k & Naphthalene & & ND & 330. $\mu$ H/kg \\
\hline Carbazole & & ND & 330. $\mu / 2 \mathrm{~kg}$ & 2-Nivomiline & & ND & 330. $48 / \mathrm{kg}$ \\
\hline 4-Culoroaniline & & ND & 330. मe/s & 3-Nibroaniline & & ND & 330. $10 / \mathrm{kg}$ \\
\hline 4-Chioro-3-medhyl & phenol & ND & 330. $\mu \mathrm{C}$ kg & 4Nitromiline & & ND & 330. $148 / \mathrm{ks}$ \\
\hline 2-Chlorosupbethalex & & ND & 330. $\mu \mathrm{g} / \mathrm{kg}$ & Nitrobenzedec & & ND & 330. $\mu \pi / \mathbf{k} 8$ \\
\hline 2-Chlorophenol & & ND & 330. pelkg & 2-Nitrophenol & & ND & 330. pgkes: \\
\hline 4-Chloropheayl phe & enyl ether & No & 330. ngtke & 4Nibropitenol & & ND & 330. He/kg \\
\hline Chrycens & & ND & 330. $\mu$ e/ks & N-Nitrosodi-a-propylami & mine & ND & 330. $\mu g / \mathrm{kg}_{\mathrm{B}}$ \\
\hline Dibenz $(2, h)$ entare & leone & ND & 330. $\mu \mathrm{g} / \mathrm{kg}$ & N-Nirosodimechylamine & & ND & 330. $\mathrm{\mu p} / \mathrm{kg}$ \\
\hline Dibenzofimn & & ND. & 330. ne/ke & N-Nirosodiphenylumine & & ND & 330. $\mathrm{\mu g} / \mathrm{kg}$ \\
\hline Di-p-butyl phthaiate & & ND & 330. $\mu g / k g$ & Pentuchlorophenol & & ND & 330. $\mu \varepsilon / \mathrm{kg}$ \\
\hline 1,2-Dichlorobenzed & de $(0-D C B)$ & ND & 330. Me/ks & Phenol & & ND & 330. $\mathrm{Rg} / \mathrm{kg}$ \\
\hline 1,3-Dichlorobenzeds & $D E(\infty D-D C B)$ & ND & 330. $\mu 8 / \mathrm{kg}$ & Phennothreme & & ND & 330. $\mathrm{Hg} / \mathrm{kg}$ \\
\hline 1,4-Dieh]oroberzen & ne (p-DCB) & ND & 330. $1 \mathrm{er} / \mathrm{cs}$ & Pyrene & & ND & $330 . \mu \mathrm{g} / \mathrm{kg}$ \\
\hline 2,4-Dichlorophenol & & ND & 330. $\mu 8 \mathrm{~kg}$ & Pyoidine & & $\begin{array}{l}\text { ND } \\
\text { ND }\end{array}$ & 330. $\mu \mathrm{g} / \mathrm{kg}$ \\
\hline $\begin{array}{l}\text { 3,3-Dichlorobernzadi } \\
\text { Diethyl phithalate } \\
\text { 2,4-Dimathytphenol }\end{array}$ & dine & $\begin{array}{l}\text { ND } \\
\text { ND } \\
\text { ND }\end{array}$ & $\begin{array}{l}330 . \mu 9 \mathrm{~kg} \\
330 . \mu \rho / k g \\
330 . \mu g / k g\end{array}$ & $\begin{array}{l}\text { 1,2,4-Trichlarobenzeale } \\
\text { 2,4,5-Trichlorophenol } \\
\text { 2,4,6-Trichlorophenol }\end{array}$ & & $\begin{array}{l}\text { ND } \\
\text { ND }\end{array}$ & 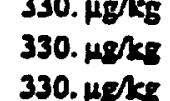 \\
\hline \multicolumn{8}{|c|}{ 2UALITY CONTROL DATA: } \\
\hline \multicolumn{3}{|l|}{ Surrogate } & \multicolumn{2}{|r|}{ \%Recover } & & \multicolumn{2}{|c|}{ Accoptable Rance } \\
\hline \multicolumn{2}{|c|}{$\begin{array}{l}\text { 3,4,6-Tribromophenol } \\
\text { 3-Fluorobiphenyl } \\
\text { 3-Fluorophenol } \\
\text { Jitrobenzene-d5 } \\
\text {-Terphenyl-d14 } \\
\text { 'henol-d6 }\end{array}$} & & & $\begin{array}{l}40 \\
56 \\
49 \\
52 \\
68 \\
56\end{array}$ & & \multicolumn{2}{|c|}{$\begin{array}{l}19-122 \\
30-115 \\
25-121 \\
23-120 \\
18-137 \\
24-113\end{array}$} \\
\hline
\end{tabular}




\begin{tabular}{|c|c|c|c|}
\hline $\begin{array}{l}\text { CLIENT: } \\
\text { PROJECT D: } \\
\text { PROJECT \#: }\end{array}$ & $\begin{array}{l}\text { Bechtel Nevada } \\
\text { V1282 } \\
23081\end{array}$ & $\begin{array}{l}\text { CLIENT D: } \\
\text { DATE SAMPLED: } \\
\text { NEL SAMPLE DD: }\end{array}$ & $\begin{array}{l}039908-0-B \\
9 / 24 / 01 \\
L 0109246-02\end{array}$ \\
\hline
\end{tabular}

TEST:

Semi-Volatile Organic Compounds by EPA 8270C, Dec, 1996

METHOD: EPA 8270

MATRE: Solid

EXTRACTED:

ANALYZED:

9/28/01

DIIUTION: 1

ANAIYST:

$10 / 8 / 01$

DIVITON: 1

\section{PARAMETER}

Acensphdheate

Acensphthyleno

Anilibe

Antbrecene

Arobensome

Benzo (s) onthrecene

Berson (B) thuoranthent

Berroo (k) Aluorenthene

Benzoie Acid

Benzo (sh,i) perylese

Benzo (a) pyrene

Benzyl alcohol

bis (2-Chloroechyl) echer

bis (2-Chloroecthaxy) methere

bis (2-ehlorossopropyl) ether

bis (2-Echy|hexyl)phechaleve

Butyl benoyl phthalate

4-Bromophenyl phenyl ether

Carbazole

4-Chlorosniline

4-Chloro-3-methyl pheaol

2-Chlorosuphehalene

2-Chlorophenol

4-Chlorophenyl pheoyl ether

Chryene

Dibenz (ah) enthracene.

Dibecorafuras

Di-n-buryl phthelate

1,2-Dichlorobenzene (0-iJCB)

1,3-Dichlorobenzene (m-DCB)

1,4-Dichlorobenzene ( $p$ - ! " :B)

2,4-Dichlorophenol

3,3'-Dichlorobenzidine

Jietbyl phthalare

2,4-Dimetbytphenol

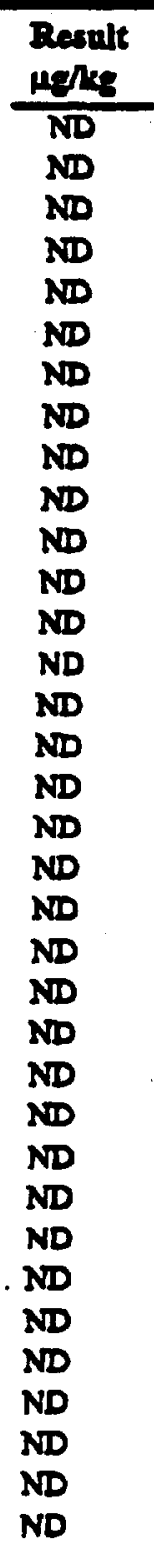

\section{Reporting}

Limit

330. $10 \mathrm{Ags}$

330. He/kg

330. Helks

330. HQk

330. Mekse

330. $\mu \mathrm{g} / \mathrm{kg}$

330. netke

330. nelks

330. मN/

330. HE/kE

330. Heles

330. $\mathrm{Ng} / \mathrm{kS}$

330. ME/RS

330. perks

330. $\mu$ glks

330. nefles

330. pekg

330. welke

330. $\mu$ elkg

330. proks

330. $\mu \mathrm{g} / \mathrm{kg}$

330. $\mu \mathrm{g} / \mathrm{KS}$

330. $\mu$ esk

330. $\mu \mathrm{N} / \mathrm{kS}$

330. wets

330. $\mu$ els

330. ugkes

330. Helke

330. $\mathrm{kg} / \mathrm{kg}$

330. $\mathrm{\mu g} / \mathrm{kg}$

330. ueves

330. He/KE

330. relks

330. nelks

330. $\mu \mathrm{g} / \mathrm{Kg}$

\section{PARAMTITER}

Disnechyl phethaiste

4,6-Dinitro-2-methyl phenol

2,4-Dinitrotoluese (DNT)

2,6-Dirimsoso:

2,-Dinitropkesol

Difn-ocoyl phtsalame

Furotenthene

Thuorene

Hexceblarobencene

Hexnebloroburadiene

Hexechlorocyclopentadiene

Hexichloroechune

Indean $(1,2,:-c, i)$ pyrene

Inophorose

2-Methyion: $:$ -

2-Methytphe

3,4-Mebylp: andi (isomarie purr)

Naphthalease

2-Nivonili=:

3-Nitroanilit:

4Nicromilisice

Nitroberezenc

2-Nitropheno1

4Nitrophenol

NoNitroeodi-i:-2-20ylamine

N-Nitrosodir : is is armine

N-Nitrosodif juirlanine

Penpechlosor... sol

Pheool

Pheasuturase

Pyrease

Pyridine

1,2,4-Trieblorsuenzene

2,4,5-Trichloropinenol

2,4,6-Trichlo:ophead

SKV - Las Vegas Divition

\begin{tabular}{|c|c|}
\hline $\begin{array}{l}\text { Rerult } \\
\text { MD/ki }\end{array}$ & $\begin{array}{l}\text { Reporting } \\
\text { Uimt }\end{array}$ \\
\hline ND & 330. $\mathrm{pg} / \mathrm{kg}$ \\
\hline ND & 330. $\mu \rho / k g$ \\
\hline ND & 330. $\mu \mathrm{g} / \mathrm{kg}$ \\
\hline ND & 330. $10 / \mathrm{kg}$ \\
\hline ND & 330. $\mu=/ \mathrm{k}$ \\
\hline ND & 330. nokse \\
\hline ND & 330. $\mathrm{M} / \mathrm{kS}$ \\
\hline ND & 330. melks \\
\hline ND & 330. $\mathrm{kg} / \mathrm{kS}$ \\
\hline ND & 330. ne/ke \\
\hline ND & 330. $\mu / / \mathrm{kg}$ \\
\hline ND & 330. neks \\
\hline ND & 330 noles \\
\hline ND & 330. $\mathrm{Hg} / \mathrm{kg}$ \\
\hline ND & 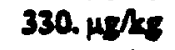 \\
\hline $\mathbf{N D}$ & 330. pe/es \\
\hline ND & 330. $19 / \mathrm{ks}$ \\
\hline ND & 330. $\mu / \mathrm{K}_{\mathrm{B}}$ \\
\hline ND & 330. He/ks \\
\hline ND & 330. $\mu \mathrm{g} / \mathrm{kg}$ \\
\hline D & 330. He/kE \\
\hline ND & 330. Me/kE \\
\hline ND & 330. pe/ke \\
\hline ND & 330. मp/es \\
\hline ND & 330. ngles \\
\hline ND & 330. $\mathrm{\mu O} / \mathrm{kg}$ \\
\hline ND & 330. $\mathrm{\mu g} / \mathrm{kg}$ \\
\hline ND & 330. ne/ks \\
\hline ND & 330. pg/ks \\
\hline ND & 330. $\mu g / k g$ \\
\hline ND & 330. po/ks \\
\hline ND & 330. $\mathrm{kg} / \mathrm{kg}$ \\
\hline ND & 330. $\mu \mathrm{g} / \mathrm{kB}$ \\
\hline ND & 330. He/kg \\
\hline ND & 330. $\mu \mathrm{g} / \mathrm{kg}$ \\
\hline
\end{tabular}

JUALTTY CONTROL DATA:

\section{iurrogate}

3,4,6-Tribramophenol

b-Fluorobiphenyl

!-Fluoropbenol

vitrobenzene-ds

1-Terphenyl-d14

'henol-d6

\begin{tabular}{c} 
\% Recovery \\
\hline 45 \\
50 \\
47 \\
48 \\
67 \\
51
\end{tabular}

\section{Acceptable Range}

19- 122

$30-115$

$25-121$

$23-120$

$18-137$

$24-113$

TD - Not Detected

This report shall not be reproduced except in full, without the written approval of the laboratory. 


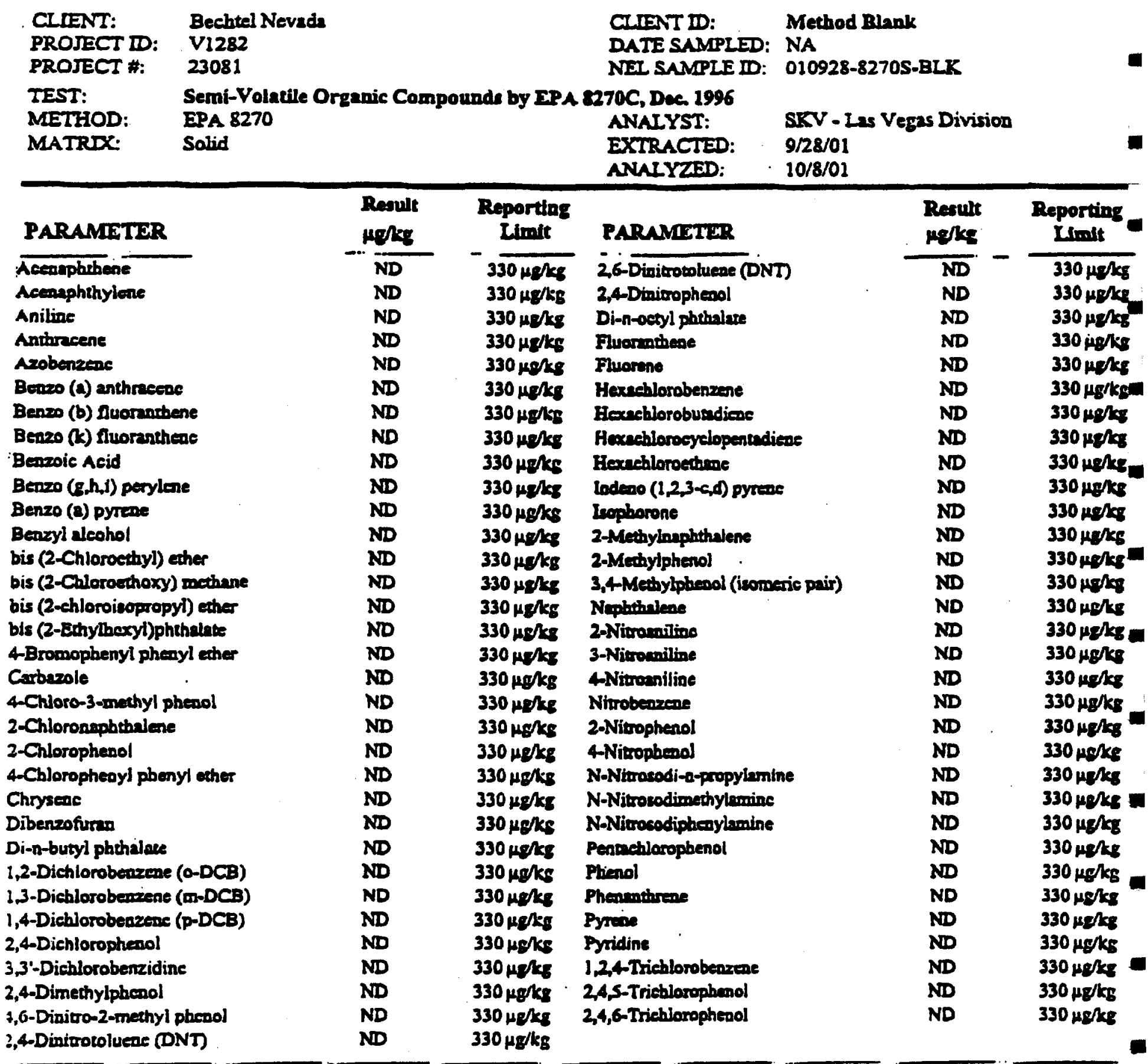

QUALITY CONTROL DATA:

Surrogate

2,4,6-Tribromophenol

2-Fluorobiphenyl

2-Fluorophenol

Virrobenzene-d5

2-Terphenyl-dl4

henol-d6

\begin{tabular}{c} 
\% Recovery \\
\hline 27 \\
58 \\
49 \\
59 \\
64 \\
59
\end{tabular}

Acceptable Range

$19-122$

$30-115$

$25-121$

$23-120$

$18-137$

$24-113$

ID - Not Detected

his report shall noz be reproduced except in full, without the written approval of the laboratory. 


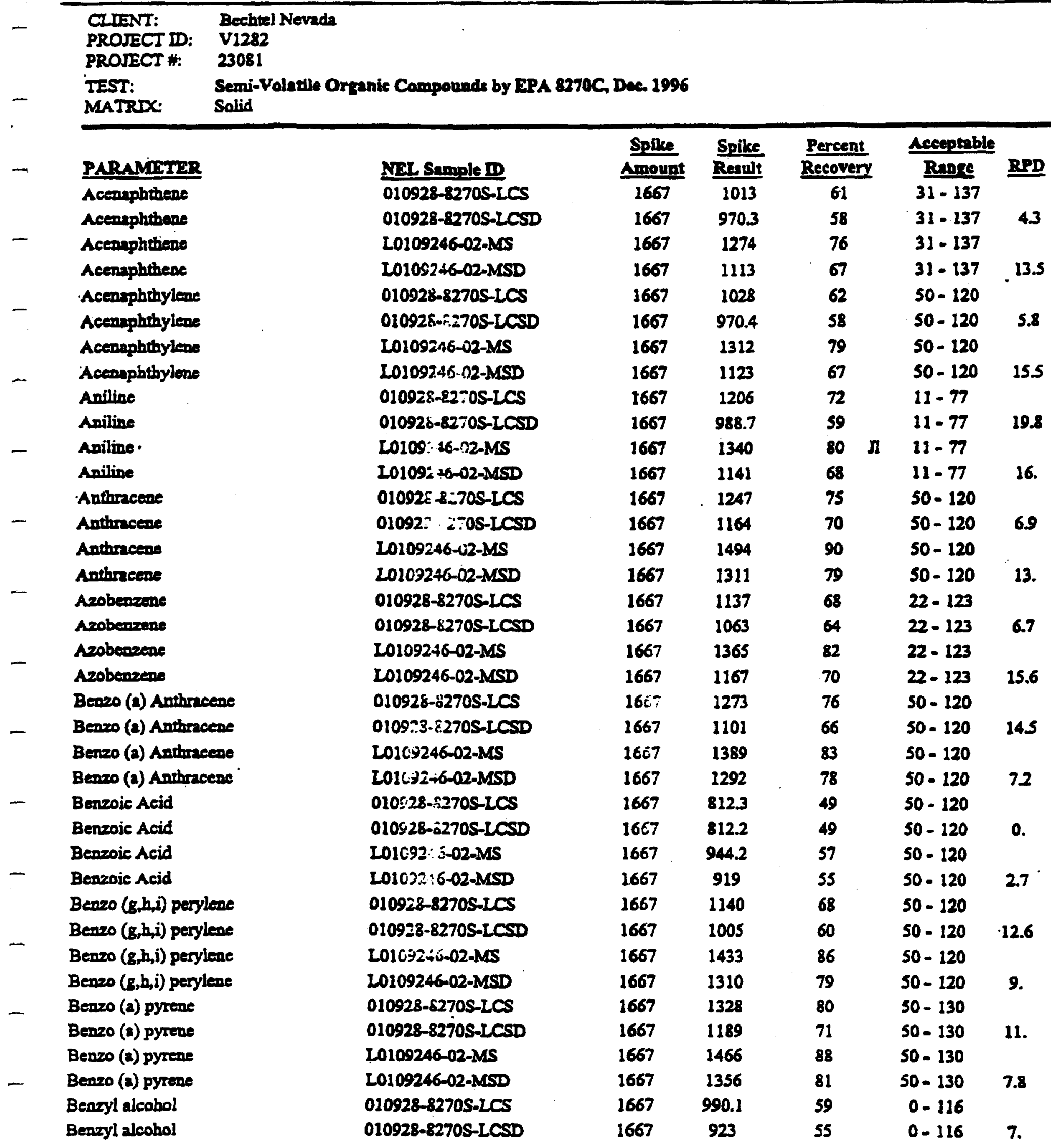

ND - Not Detected

This report shall nos be reproduced except in full, without the written approval of the laboratony. 


$\begin{array}{ll}\text { CIIENT: } & \text { Bechtel Nevada } \\ \text { PROIECT ID: } & \text { V1282 } \\ \text { PROJECT \#: } & 23081 \\ \text { TEST: } & \text { Semi-Volatile Oreanic Compounds by EPA 8270C, Dec } 1996 \\ \text { MATREX: } & \text { Solid }\end{array}$

\begin{tabular}{|c|c|c|c|c|c|c|}
\hline PARAMETER & Nin Sample ID & $\frac{\text { Splke }}{\text { Anownt }}$ & $\frac{\text { Spiles }}{\text { Result }}$ & $\frac{\text { Percent }}{\text { Recovery }}$ & $\frac{\text { Acesptable }}{\text { Range }}$ & RPD \\
\hline Benzyl alcohol & L0109246-02-MS & 1667 & 1248 & 75 & $0-116$ & \\
\hline Benzyl alcohol & L0109246-02-MSD & 1667 & 1062 & 64. & $0-116$ & 16. \\
\hline bis (2-Chloroethyl) ether & 010928-8270S-LCS & 1667 & 896.9 & 54 & $15-107$ & \\
\hline bis (2-Chlorocthyl) ether & 010928-8270S-LCSD & 1667 & 904.8 & 54 & $15-107$ & 0.9 \\
\hline bis (2-Chioroethyl) ether & L0109246-02-MAS & 1667 & 1151 & 69 & $15-107$ & \\
\hline bis (2-Chloroethyl) ether & L0109246-02-MSD & 1667 & 951.23 & 57 & $15-107$ & 19. \\
\hline bis (2-Chloroethoxy) methine & 010928-8270S-LCS & 1667 & 978.6 & 59 & $26-97$ & 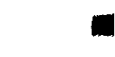 \\
\hline bis (2-Chloroethoxy) methane & 010928-8270S-LCSD & 1667 & 929.3 & 56 & $26-97$ & 5.2 \\
\hline bis (2-Chloroethoxy) methene & L0109246-02-MS & 1667 & 1193 & 72 & $26-97$ & \\
\hline bis (2-Chlorocthoxy) methane & L0109246-02-MSD & 1667 & 1023 & 61 & $26-97$ & $153^{3}$ \\
\hline bis (2-chloroisogropyl) ether & 010928-8270S-LCS & 1667 & 940.3 & 56 & $50-120$ & \\
\hline bis (2-chlaroisopropyl) ether & 010928-8270S-LCSD & 1667 & 925.8 & 56 & $50-120$ & 1.6 \\
\hline bis (2-chloroisopropyl) ether & L0109246-02-MS & 2667 & 1169 & 70 & $50 \cdot 120$ & \\
\hline bis (2-chloroisopropyl) ether & L0109246-02-MSD & 1667 & 978.1 & 59 & $50 \cdot 120$ & 17.8 \\
\hline bis (2-Ethylhexyl)phithalate & 010928-8270S-LCS & 1667 & 1335 & 80 & $50-120$ & \\
\hline bis (2-Ethylhexyl)plethelate & 010928-8270S-ICSD & 1667 & 1156 & 69 & $50-120$ & 14.4 \\
\hline bis (2-Ethylhexyl)phrbslate & L0109246-02-MS & 1667 & 1439 & 86 & $50-120$ & \\
\hline bis (2-Ethylhexyl)phthalate & L0109246-02-MSD & 1667 & 1385 & 83 & $50-120$ & 3.8 \\
\hline Butyl benzyl phthalnie & 010928-8270S-LCS & 1667 & 1312 & 79 & $50-120$ & \\
\hline Butyl benzyl phethriese & 010928-8270S-LCSD & 1667 & 1155 & 69 & $50-120$ & 12.7 \\
\hline Butyl benzyl phthalate & L0109246-02-MS & 1667 & 1406 & 84 & $50-120$ & \\
\hline Butyl benzyl phthalate & L0109246-02-MSD & 1667. & 1334 & 80 & $50-120$ & 5.3 \\
\hline 4-Bromophenyl phenyl ether & 010928-8270S-LCS & 1667 & 1253 & 75 & $50-120$ & \\
\hline 4-Bromopheny! phenyl ether & 010928-8270S-LCSD & 1667 & 1147 & 69 & $50-120$ & 8.8 \\
\hline 4-Bromophenyl phenyl ether & L0109246-02-MS & 1667 & 1497 & 90 & $50-120$ & \\
\hline 4-Bromophenyl phenyl ether & L0109246-02-MSD & 1667 & 1331 & 80 & $50-120$ & 11.7 \\
\hline 4-Chloroaniline & 010928-8270S-ICS & 1667 & 793.8 & 48 & 1.68 & \\
\hline 4-Chloroaniline & 010928-8270S-LCSD & 1667 & 596.6 & 36 & 1.68 & $28.4^{\circ}$ \\
\hline 4-Chloroaniline & L0109246-02-MS & 1667 & 769.4 & 46 & $1-68$ & \\
\hline 4-Chloroaniline & L0109246-02-MSD & 1667 & 710.2 & 43 & $1-68$ & 8. \\
\hline 4-Chloro-3-methyl pheal & 010928-8270S-LCS & 1667 & 1114 & 67 & $26-103$ & \\
\hline 4-Chloro-3-methyl phenol & 010928-8270S-LCSD & 1667 & 1044 & 63 & $26-103$ & 6.5 \\
\hline 4-Chloro-3-methyl phenol & L0109246-02-MS & 1667 & 1472 & 88 & $26-103$ & \\
\hline 4-Chloro-3-mothyl phenol & L0109246-02-MSD & 1667 & 1202 & 72 & $26-103$ & 20.2 \\
\hline 2-Chloronaphethalene & 010928-8270S-LCS & 1667 & 1000 & 60 & $50-120$ & \\
\hline 2-Chloramaphthalene & 010928-8270S-LCSD & 1667 & 1011 & 61 & $50-120$ & 1.1 \\
\hline 2-Chloronnphthalene & L0109246-02-MS & 1667 & 1297 & 78 & $50-120$ & \\
\hline 2-Chloronaphthalene & L0109246-02-MSD & 1667 & 1099 & 66 & $50-120$ & 16.5 \\
\hline
\end{tabular}

ND - Not Detected

This report shall not be reproduced except in full, without the written approval of the laborasory. 
NEL LABORATORIES

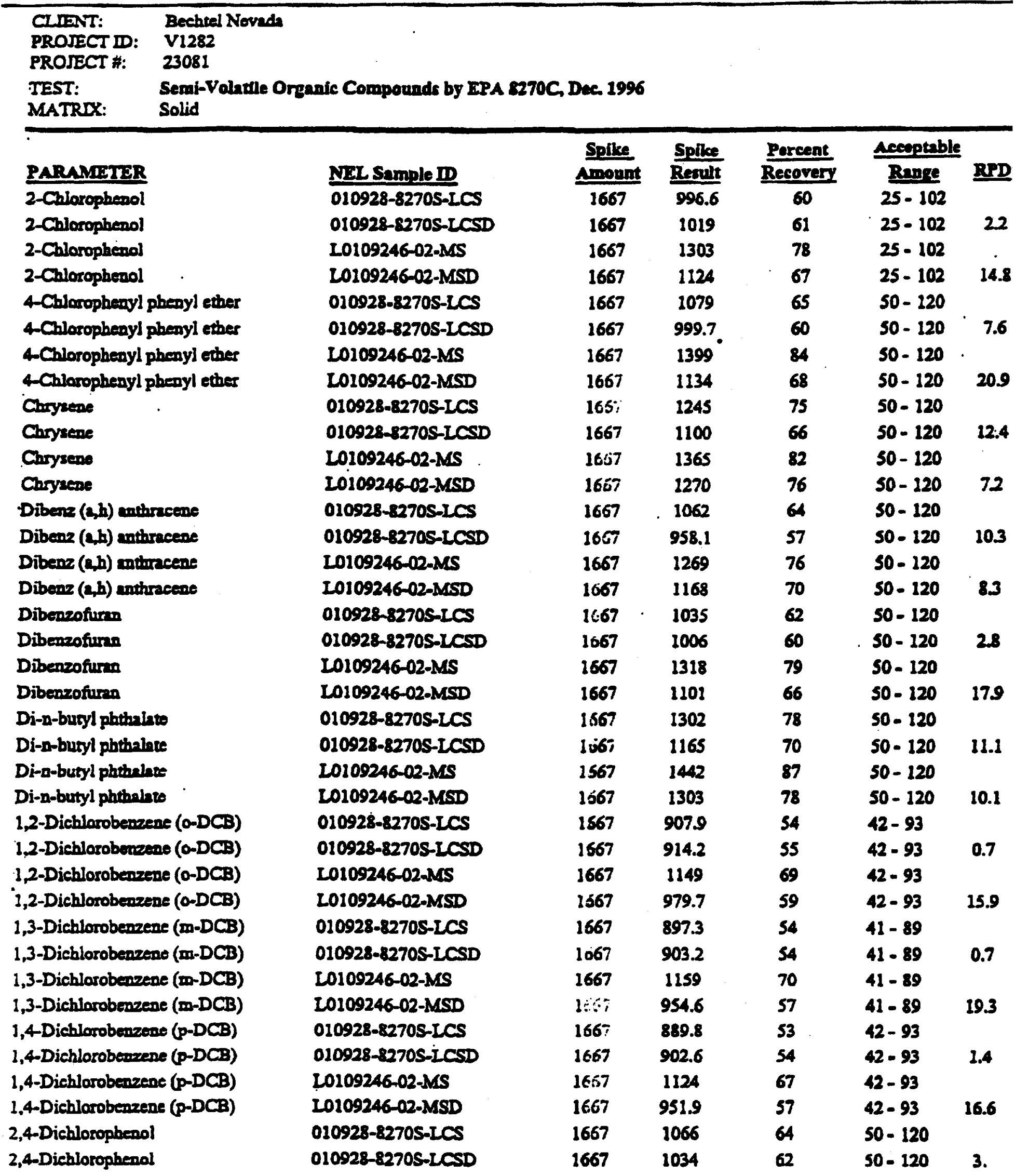

ND - Not Detected

This repors shall not be reproduced except in full, withous the written approval of the laboratory. 


\begin{tabular}{ll}
\hline CIIENT: & Bechtel Nevads \\
PROJECT D: & V1282 \\
PROJECT $*:$ & 23081 \\
TEST: & Semi-Volatle Organic Compounds by EPA 8270 C, Dec. 1996 \\
MATRIX: & Solid
\end{tabular}

\begin{tabular}{|c|c|c|c|c|c|c|}
\hline PARAMETER & NEL Sample ID & Splke & Splle & $\frac{\text { Percent }}{\text { Recovery }}$ & $\frac{\text { Acceptable }}{\text { Ranee }}$ & $\underline{\mathbf{R P D}}$ \\
\hline 2,4-Dichlorophenol & L0109246-02-MS & 1667 & 1408 & 84 & $50-120$ & \\
\hline 2,4-Dichlorophenol & L0109246-02-MSD & 1667 & 1206 & 72 & $50-120$ & \\
\hline 3,3'-Dichlarobenricitive & 010928-8270S-LCS & 1667 & 973.9 & 58 & $11 \cdot 85$ & \\
\hline 3,3'-Dichlorobensidine & 010928-8270S-LCSD & 1667 & 724.4 & 43 & $11-85$ & \\
\hline 3,3'-Dichlarobereaidine & L0109246-02-MS & 1667 & 962.4 & 58 & $11-85$ & \\
\hline 3,3'-Dichlaroberasidine & L0109246-02-MSD & 1667 & 896.9 & 54 & $11-85$ & $\cdot$ \\
\hline Diethyl Phthaluete & 010928-8270S-LCS & 1667 & 1293 & 72 & $50-120$ & \\
\hline Diechyl Phthinte & 010928-8270S-LCSD & 1667 & 1071 & 64 & $50-120$ & 10.8 \\
\hline Diethyl phthrinte & L0109246-02-MS & 1667 & 1379 & 83 & $50-120$ & \\
\hline Diethyl phthalate & L0109246-02-MSD & 1667 & 1223 & 73 & $50-120$ & \\
\hline 2,4-Dimethylphenol & 010928-8270S-LCS & 1667 & 592.2 & 36 & $50-120$ & \\
\hline 2,4-Dimethylphenol & 010928-8270S-LCSD & 1667 & 470.2 & 28 & $50-120$ & 23. \\
\hline 2,4-Dimethytphenol & L0109246-02-MS & 1667 & 1214 & 73 & $50-120$ & \\
\hline 2,4-Dimethylphenol & L0109246-02-MSD & 1667 & 916 & S5 & $50-120$ & 28. \\
\hline Dimethyl phthalare & 010928-8270S-LCS & 1667 & 1111 & 67 & $50-120$ & \\
\hline Dimethyl phothalate & 010928-8270S-LCSD & 1667 & 1073 & 64 & $50-120$ & \\
\hline Dimethyl phthalate & L0109246-02-MS & 1667 & 1409 & 85 & $50-120$ & \\
\hline Dimethyl phthalate & L0109246-02-MSD & 1667 & 1162 & 70 & $50-120$ & \\
\hline 4,6-Dinitro-2-methyl phenol & 010928-8270S-LCS & 1667 & 993.7 & 60 & $20-120$ & \\
\hline 4,6-Dinitro-2-methyl phenol & 010928-8270S-LCSD & 1667 & 880 & 53 & $20-120$ & 12.1 \\
\hline 4,6-Dinitro-2-methyl phenol & L0109246-02-MS & 1667 & 849.9 & 51 & $20 \cdot 120$ & \\
\hline 4,6-Dinitro-2-methyl phenol & L0109246-02-MSD & 1667 & 1223 & 73 & $20-120$ & 36. \\
\hline 2,4-Dinitromliene (DNI) & 010928-8270S-LCS & 1667 & 1180 & 71 & $50-111$ & \\
\hline 2,4-Dinitrotoluene (DNI) & 010928-8270S-LCSD & 1667 & 1056 & 63 & $50-111$ & \\
\hline 2,4-Dinitrotoluane (DNI) & L0109246-02-MS & 1667 & 1389 & 83 & $50-111$ & \\
\hline 2,4-Dinitroroluene (DNT) & L0109246-02-MSD & 1667 & 1196 & 72 & $50-111$ & 14.9 \\
\hline 2,6-Dinitrotoluene (DNT) & 010928-8270S-LCS & 1667 & 1096 & 66 & $50-120$ & \\
\hline 2,6-Dinitrotaluene (DNT) & 010928-8270S-LCSD & 1667 & 1023 & 61 & $50-120$ & 6.9 \\
\hline 2,6-Dinitrocoluene (DNT) & L0109246-02-MS & 1667 & 1329 & 80 & $50-120$ & \\
\hline 2,6-Dinitrotoluene (DNT) & L0109246-02-MSD & 1667 & 1176 & 71 & $50-120$ & 122 \\
\hline 2,4-Dinitrophenol & 010928-8270S-LCS & 1667 & 792.9 & 48 & $20-120$ & \\
\hline 2,4-Dinitrophenol & 010928-8270S-LCSD & 1667 & 750.4 & 45 & $20-120$ & 5.5 \\
\hline 2,4-Dinitrophenol & L0109246-02-MS & 1667 & 1004 & 60 & $20-120$ & \\
\hline 2,4-Dinitrophenol & L0109246-02-MSD & 1667 & 977.4 & 59 & $20-120$ & 2.7 \\
\hline Di-n-octyl phehnlate & 010928-8270S-LCS & 1667 & 1375 & 82 & $50-120$ & \\
\hline Dl-n-octyl phthalase & 010928-8270S-LCSD & 1667 & 1194 & 72 & $50-120$ & 14.1 \\
\hline Ji-n-octyl phthalate & L0109246-02-MS & 1667 & 1554 & 93 & $50-120$ & \\
\hline Jj-p-octyl phethalate & L0109246-02-MSD & 1667 & 1431 & 86 & $50-120$ & 8.2 \\
\hline
\end{tabular}

VD - Not Detected

This report shall not be reproduced except in full, whthous the writton approval of the laborasory. 
NEL LABORATORIES

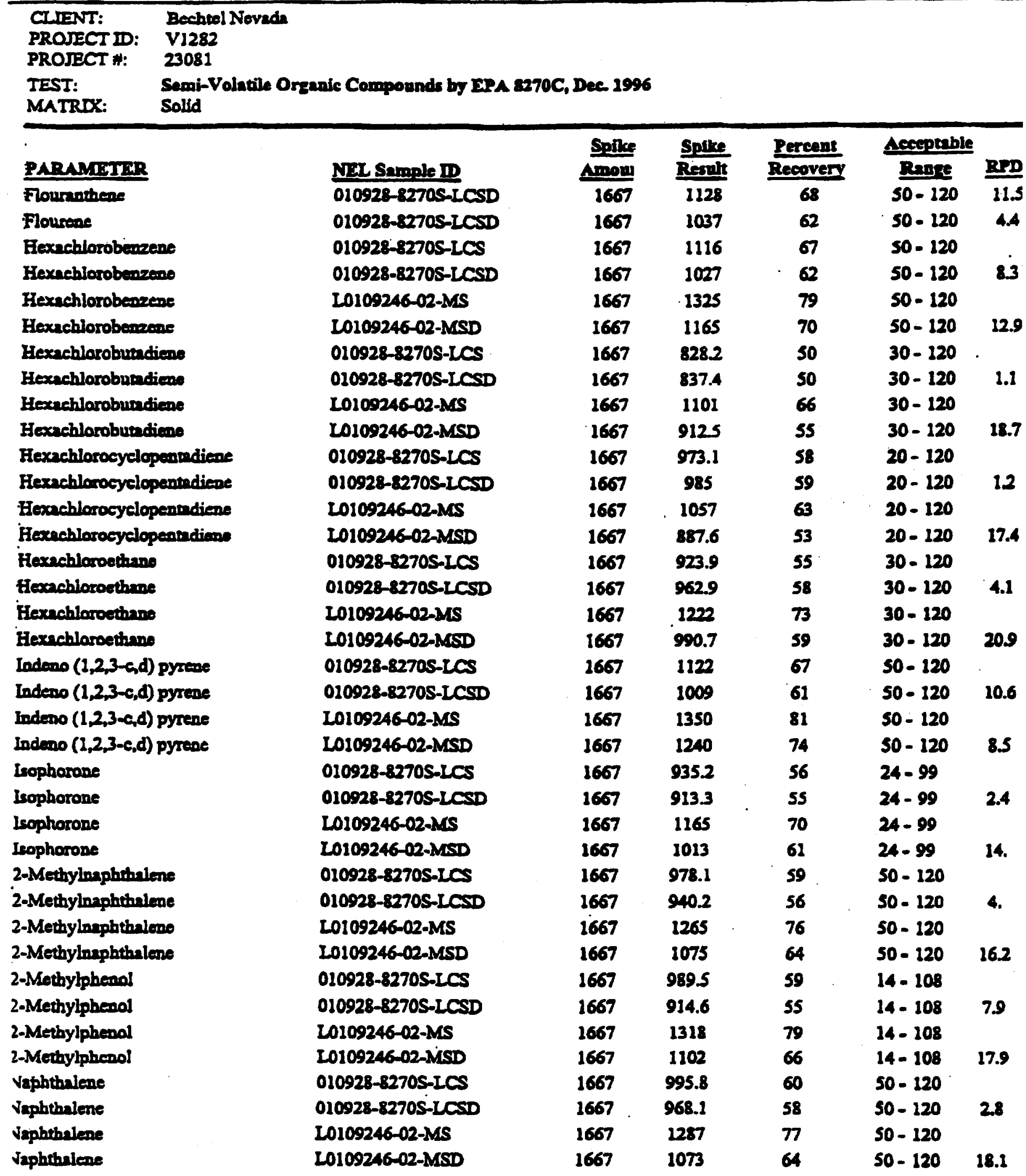

ND - Not Detected

This report shall not be reproduced except in full, withour the wrizen approval of the laboratory. 


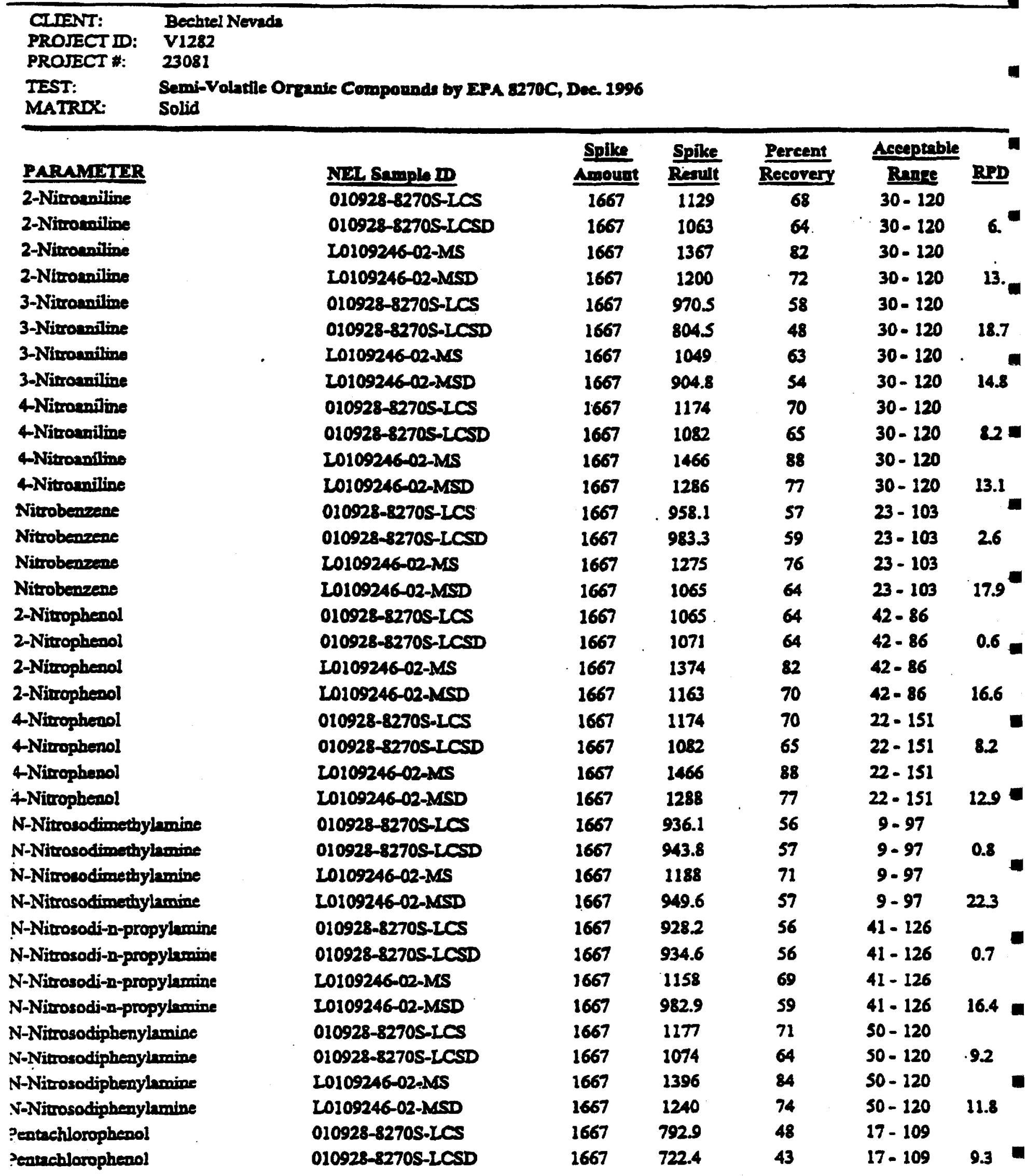

VD - Not Deteeted

This report shall not be reproduced excopt in fill, without the written cpproval of the laboratory. 
NEL LABORATORIES

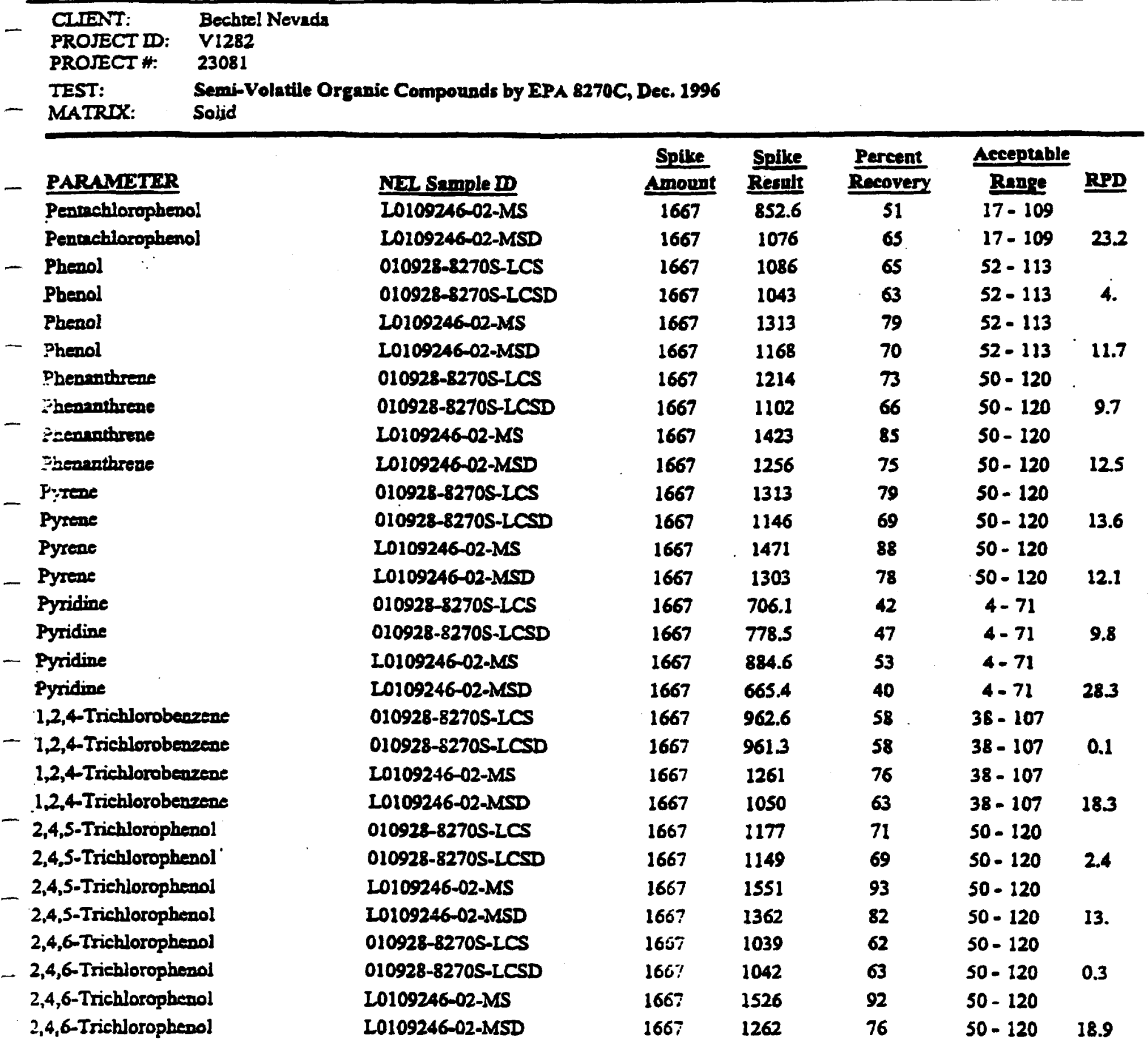

in - Not Lelecteo

his report shall not be reproduced except in full, withour the writuen approval of the laboratory. 


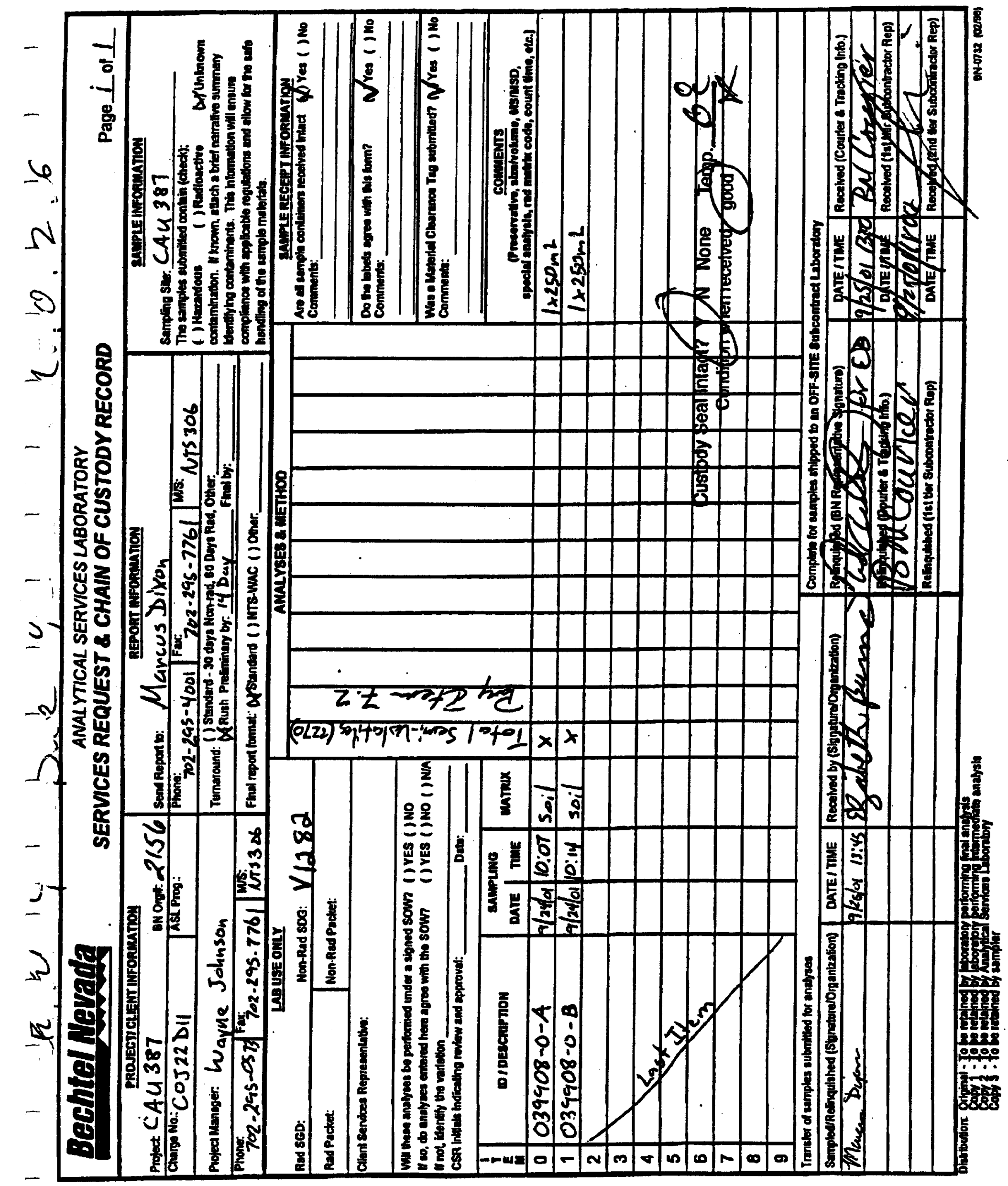




$\begin{array}{lll}\text { CLIENT: } & \text { Bechtel Nevada } \\ & & \text { P.O. Box } 98521, \text { M/S NTS273 } \\ & \text { Las Vegas, NV 89193-8521 } \\ & \text { ATTN: } & \text { Ted Redding }\end{array}$

PROJECT NAME: $\quad$ V1236

PROJECT NUMBER: 23081

Attached are the analytical results for samples in support of the above referenced project.

Samples submitted for this project were not sampled by NEL Laboratories. Samples were received by NEL in good condition, under chain of custody on 8/14/01.

Should you have any questions or comments, please feel free to contact our Client Services department at (702) 657-1010.

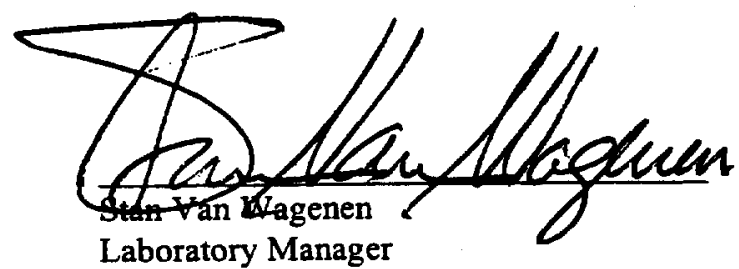

CERTIFICATIONS:

\begin{tabular}{llll}
\hline & Reno & Las Vegas & S. California \\
\cline { 2 - 4 } $\begin{array}{l}\text { Arizona } \\
\text { California }\end{array}$ & 170520 & AZ0518 & AZ0605 \\
$\begin{array}{l}\text { US Army Corps } \\
\text { of Engineers }\end{array}$ & Certified & Certified & 2264 \\
& & &
\end{tabular}

Idaho Montana Nevada L.A.C.S.D.
NEL ORDER ID: L0108129
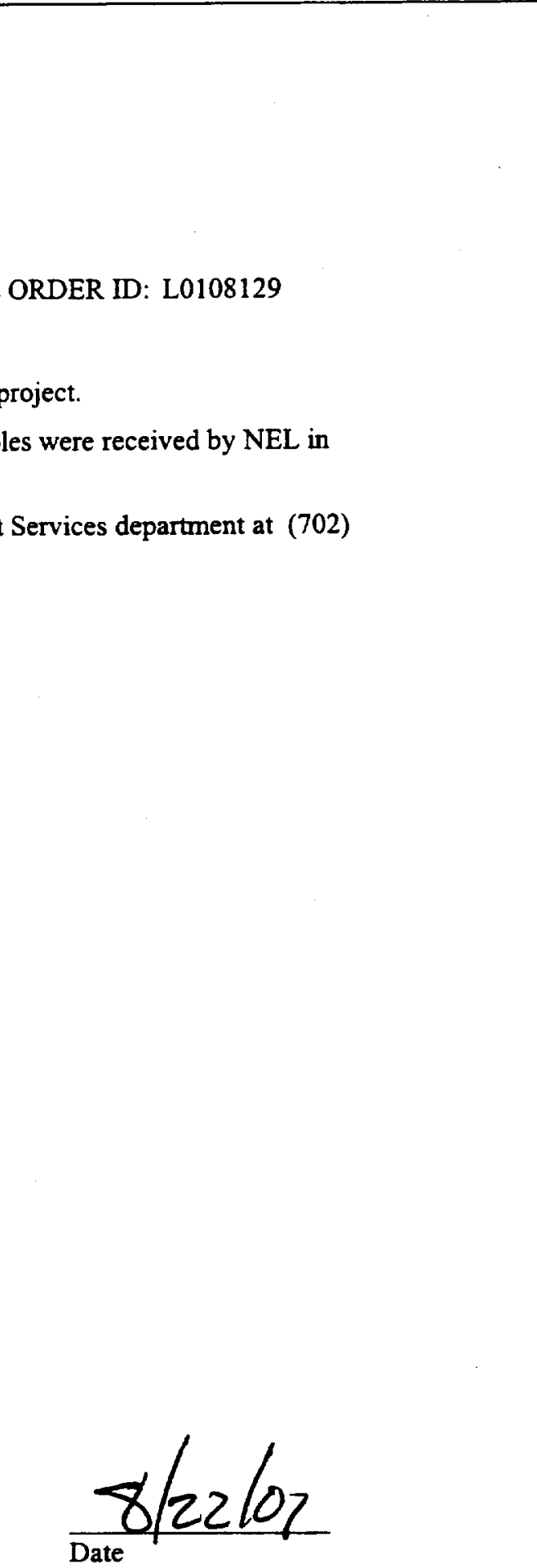

Date 
NEL LABORATORIES

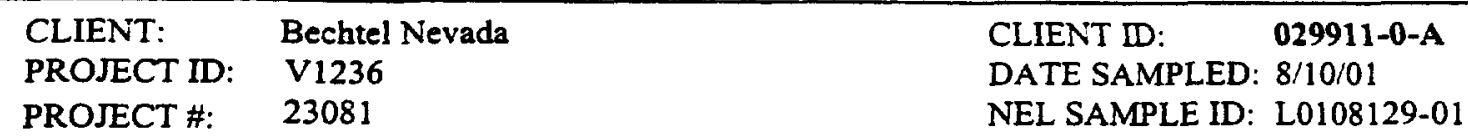

TEST: $\quad$ Total Extractable Petroleum Hydrocarbons Fuel Finger Print by EPA Method 8015M, July 1992

METHOD: EPA 8015M ANALYST: PXC - Division

MATRIX: Solid $\quad$ EXTRACTED: 8/17/01

DILUTION: $1 \quad$ ANALYZED: $\quad 8 / 21 / 01$

\begin{tabular}{lcc}
\hline PARAMETER & Result & Reporting \\
\cline { 2 - 3 } & $\mathrm{ND}$ & $\frac{\text { Limit }}{10 . \mathrm{mg} / \mathrm{kg}}$ \\
Gasoline Range (C8-C12) & $\mathrm{ND}$ & $10 . \mathrm{mg} / \mathrm{kg}$ \\
Diesel Range (C12-C22) & $\mathrm{ND}$ & $50 . \mathrm{mg} / \mathrm{kg}$ \\
Oil Range (C22-C34) & $\mathrm{ND}$ & $10 . \mathrm{mg} / \mathrm{kg}$ \\
\hline Total & M
\end{tabular}

QUALITY CONTROL DATA:

Surrogate

$\%$ Recovery

Octacosane

74

Acceptable Range

$54-130$

ND - Not Detected

This report shall not be reproduced except in full, without the written approval of the laboratory. 
NEL LABORATORIES

\begin{tabular}{lll}
\hline CLIENT: & Bechtel Nevada & CLIENT ID: 02991 1-0-B \\
PROJECT ID: & V1236 & DATE SAMPLED: 8/10/01 \\
PROJECT \#: & 23081 & NEL SAMPLE ID: L0108129-02
\end{tabular}

\section{- TEST:}

METHOD MATRIX:

DILUTION:
Total Extractable Petroleum Hydrocarbons Fuel Finger Print by EPA Method 8015M, July 1992 EPA 8015M Solid 1
ANALYST: PXC - Division

EXTRACTED: $\quad 8 / 17 / 01$

ANALYZED: $\quad 8 / 21 / 01$

\section{PARAMETER}

Gasoline Range (C8-C12)

- Diesel Range (C12-C22)

Oil Range (C22-C34)

Total

- QUALITY CONTROL DATA:

Surrogate

Octacosane
Result

ND

ND

ND

ND

\% Recovery

91
Reporting

Limit

10. $\mathrm{mg} / \mathrm{kg}$

10. $\mathrm{mg} / \mathrm{kg}$

50. $\mathrm{mg} / \mathrm{kg}$

10. $\mathrm{mg} / \mathrm{kg}$

Acceptable Range

$54-130$

- ND - Not Detected

This report shall not be reproduced except in full, without the written approval of the laboratory. 
NEL LABORATORIES

\begin{tabular}{|c|c|c|}
\hline $\begin{array}{l}\text { CLIENT: } \\
\text { PROJECT ID: } \\
\text { PROJECT \#: }\end{array}$ & $\begin{array}{l}\text { Bechtel Nevada } \\
\text { V1236 } \\
23081\end{array}$ & $\begin{array}{ll}\text { CLIENT ID: } & \text { 029911-0-C } \\
\text { DATE SAMPLED: } & 8 / 10 / 01 \\
\text { NEL SAMPLE ID: } & \text { L0108129-03 }\end{array}$ \\
\hline
\end{tabular}

TEST: $\quad$ Total Extractable Petroleum Hydrocarbons Fuel Finger Print by EPA Method 8015M, July 1992

METHOD: $\quad$ EPA 8015M

MATRIX: Solid

DILUTION: 1 ANALYST: $\quad$ PXC - Division

EXTRACTED: $\quad 8 / 17 / 01$

ANALYZED: $\quad 8 / 21 / 01$

\section{PARAMETER}

Gasoline Range (C8-C12)

Diesel Range (C12-C22)

Oil Range (C22-C34)

Total

QUALITY CONTROL DATA:

Surrogate

Octacosane
\% Recovery

83
Reporting

Limit

10. $\mathrm{mg} / \mathrm{kg}$

10. $\mathrm{mg} / \mathrm{kg}$

50. $\mathrm{mg} / \mathrm{kg}$

10. $\mathrm{mg} / \mathrm{kg}$

ND

ND

Acceptable Range

$54-130$

ND - Not Detected

This report shall not be reproduced except in full, without the written approval of the laboratory. 
NEL LABORATORIES

\begin{tabular}{lll}
\hline CLIENT: & Bechtel Nevada & CLIENT ID: \\
PROJECT ID: & V1236 & DATE SAMPLED: $8 / 10 / 01$ \\
PROJECT \#: & 23081 & NEL SAMPLE ID: $0108129-04$
\end{tabular}

- TEST:

METHOD: EPA 8015M

MATRIX: Solid

DILUTION: 1

PARAMETER

Gasoline Range (C8-C12)

Diesel Range (C12-C22)

Oil Range (C22-C34)

Total

QUALITY CONTROL DATA:

Surrogate

Octacosane
$\%$ Recovery

81
Reporting

Limit

10. $\mathrm{mg} / \mathrm{kg}$

10. $\mathrm{mg} / \mathrm{kg}$

50. $\mathrm{mg} / \mathrm{kg}$

10. $\mathrm{mg} / \mathrm{kg}$

ND - Not Detected

This report shall not be reproduced except in full, without the written approval of the laboratory.

Acceptable Range

$54-130$ 
NEL LABORATORIES

\begin{tabular}{|c|c|c|}
\hline $\begin{array}{l}\text { CLIENT: } \\
\text { PROJECT ID: } \\
\text { PROJECT \#: }\end{array}$ & $\begin{array}{l}\text { Bechtel Nevada } \\
\text { V1236 } \\
23081\end{array}$ & $\begin{array}{ll}\text { CLIENT ID: } & \mathbf{0 2 9 9 1 1 - 0 - E} \\
\text { DATE SAMPLED: } & 8 / 10 / 01 \\
\text { NEL SAMPLE ID: } & \text { L0108129-05 }\end{array}$ \\
\hline
\end{tabular}

TEST: $\quad$ Total Extractable Petroleum Hydrocarbons Fuel Finger Print by EPA Method 8015M, July 1992

METHOD: EPA 8015M ANALYST: PXC - Division

MATRIX: Solid $\quad$ EXTRACTED: 8/17/01

DILUTION: 1 ANALYZED: $8 / 21 / 01$

\begin{tabular}{llc}
\hline PARAMETER & Result & Reporting \\
\cline { 2 - 3 } Gasoline Range (C8-C12) & LD & $\frac{\text { Limit }}{10 . \mathrm{mg} / \mathrm{kg}}$ \\
Diesel Range (C12-C22) & ND & $10 . \mathrm{mg} / \mathrm{kg}$ \\
Oil Range (C22-C34) & ND & $50 . \mathrm{mg} / \mathrm{kg}$ \\
Total & ND & $10 . \mathrm{mg} / \mathrm{kg}$ \\
\hline \hline
\end{tabular}

QUALITY CONTROL DATA:

Surrogate

\% Recovery

Acceptable Range

Octacosane

87

$54-130$

ND - Not Detected

This report shall not be reproduced except in full, without the written approval of the laboratory. 
NEL LABORATORIES

\begin{tabular}{lll}
\hline CLIENT: & Bechtel Nevada & CLIENT ID: $\quad$ 029903-0-A \\
PROJECT ID: & V1236 & DATE SAMPLED: 8/10/01 \\
PROJECT \#: & 23081 & NEL SAMPLE ID: L0108129-06
\end{tabular}

- TEST:

METHOD: EPA 8015M

MATRIX: Solid

DILUTION: 1

EXTRACTED: 8/17/01

$-$

PARAMETER

Gasoline Range (C8-C12)

- Diesel Range (C12-C22)

Oil Range (C22-C34)

Total

QUALITY CONTROL DATA:

Surrogate

Octacosane

\% Recovery

97
Reporting

Limit

10. $\mathrm{mg} / \mathrm{kg}$

10. $\mathrm{mg} / \mathrm{kg}$

50. $\mathrm{mg} / \mathrm{kg}$

10. $\mathrm{mg} / \mathrm{kg}$

ND - Not Detected

This report shall not be reproduced except in full, without the written approval of the laboratory. 
NEL LABORATORIES

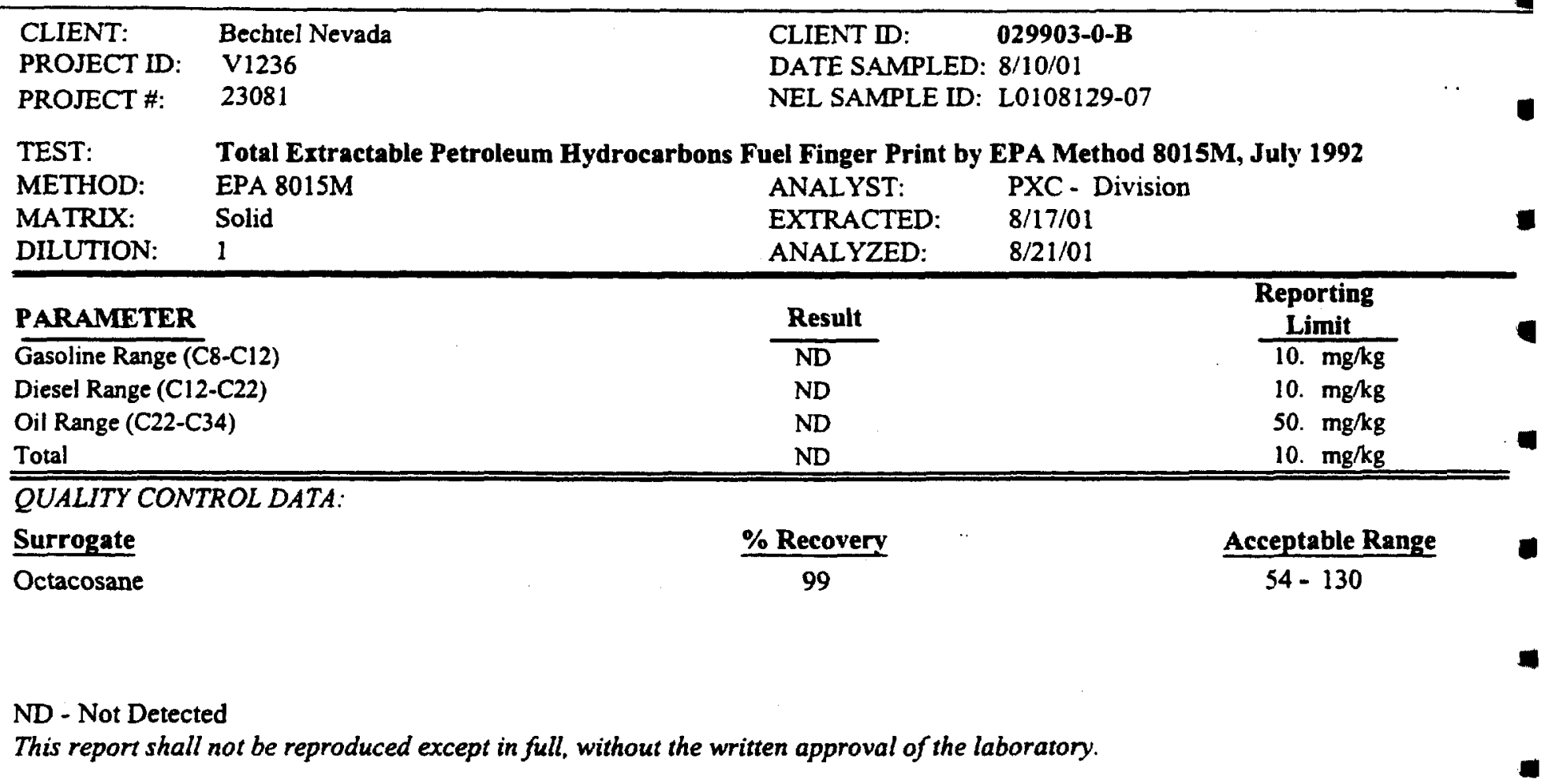


NEL LABORATORIES

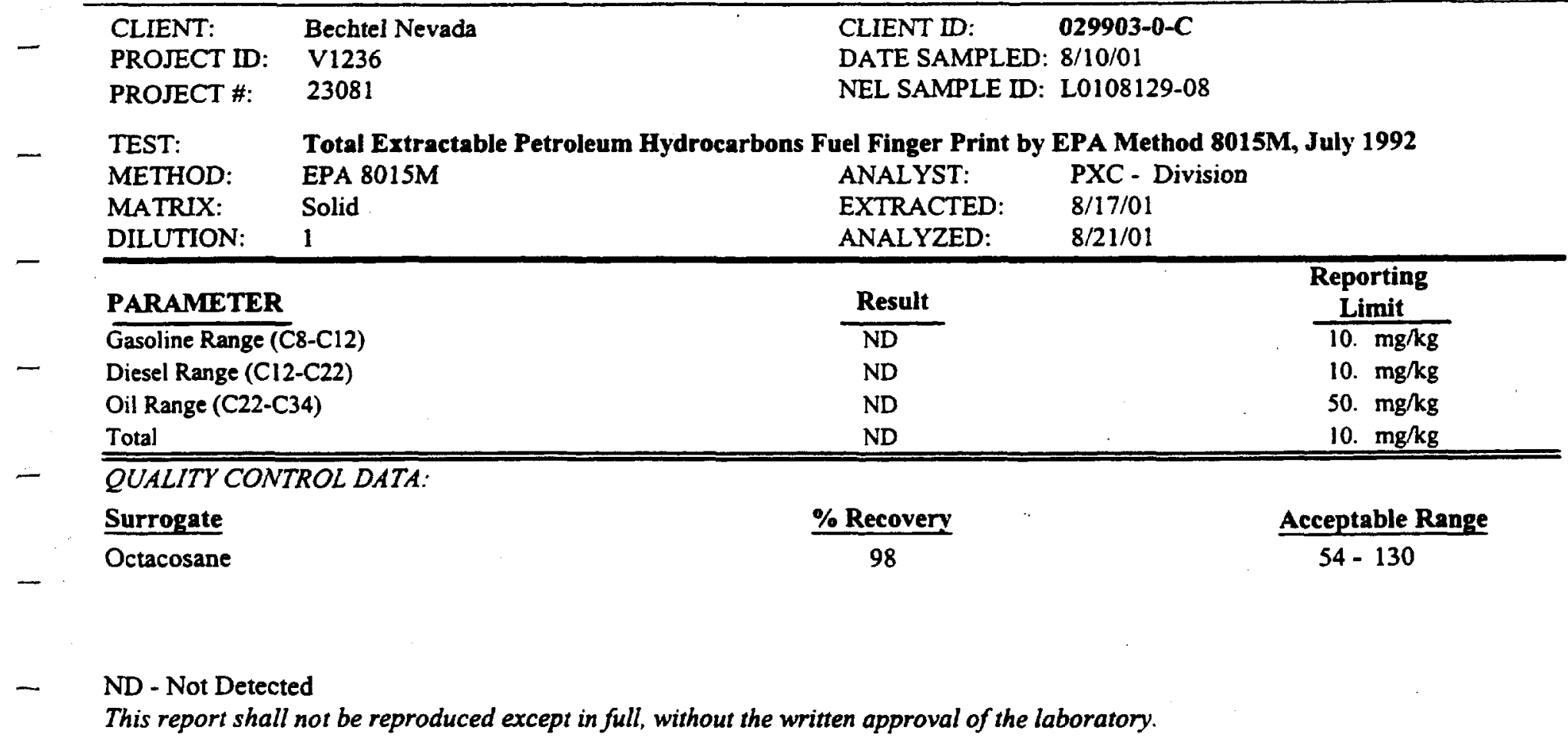


NEL LABORATORIES

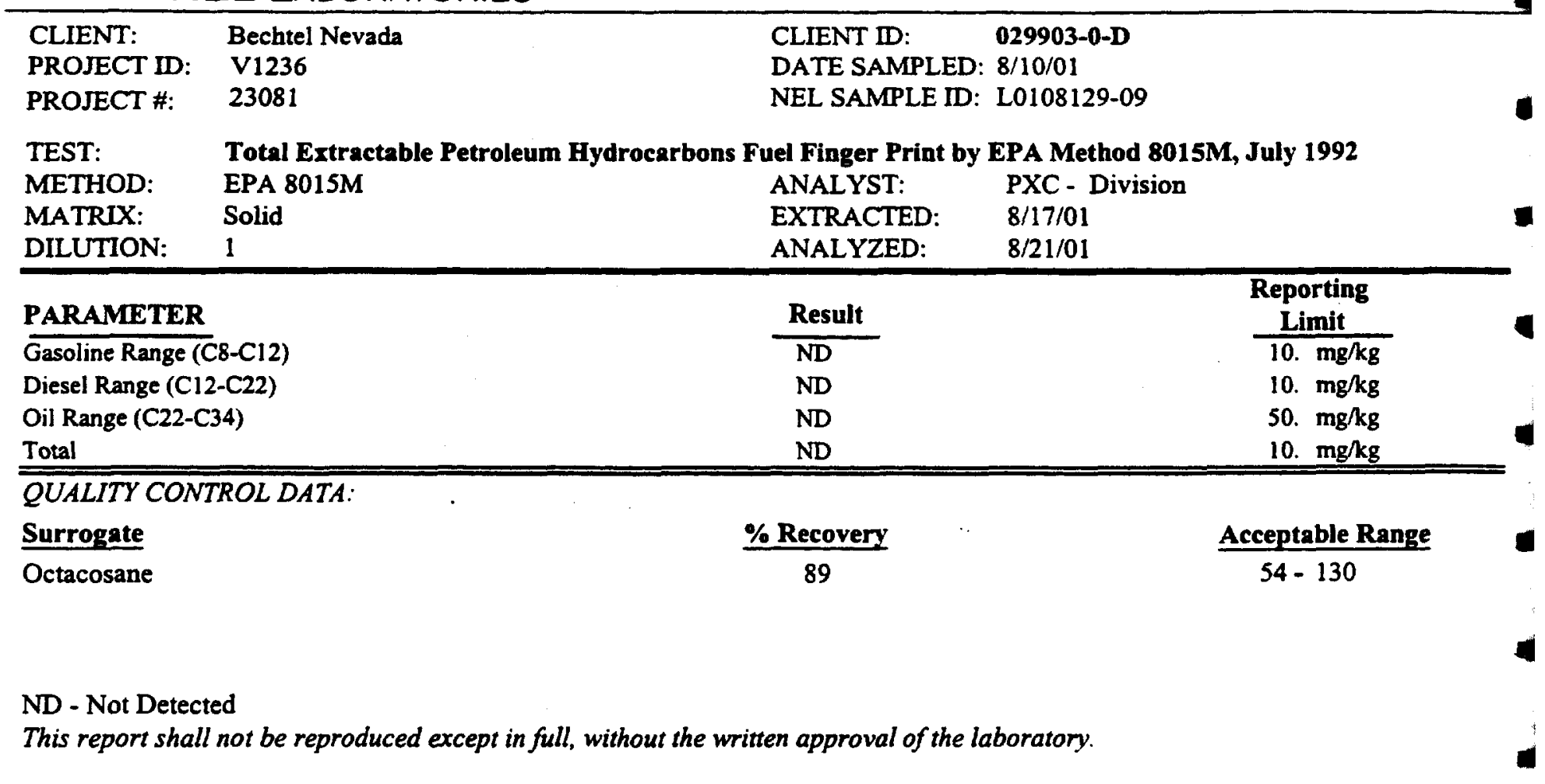


NEL LABORATORIES

\begin{tabular}{llll}
\hline CLIENT: & Bechtel Nevada & CLIENT ID: & Method Blank \\
PROJECT ID: & V1236 & DATE SAMPLED: & NA \\
PROJECT \#: & 23081 & NEL SAMPLE D: & 010817TPHS-FP-BLK
\end{tabular}

\section{- TEST: METHOD: $\quad$ EPA 8015M}

MATRIX: Solid
PARAMETER

Gasoline Range (C8-C12)

Diesel Range (C12-C22)

Oil Range (C22-C34)

Total
Total Extractable Petroleum Hydrocarbons Fuel Finger Print by EPA Method 8015M, July 1992 ANALYST: $\quad$ SKV - Las Vegas Division EXTRACTED: $\quad 8 / 17 / 01$ ANALYZED: $\quad 8 / 21 / 01$

\section{QUALITY CONTROL DATA:}

Surrogate

Octacosane
\% Recovery

96
Reporting

Limit

10. $\mathrm{mg} / \mathrm{kg}$

10. $\mathrm{mg} / \mathrm{kg}$

50. $\mathrm{mg} / \mathrm{kg}$

10. $\mathrm{mg} / \mathrm{kg}$

ND - Not Detected

This report shall not be reproduced except in full, without the written approval of the laboratory. 


\section{CLIENT: Bechtel Nevada}

PROJECT ID: V1236

PROJECT \#: 23081

TEST: Total Extractable Petroleum Hydrocarbons by EPA Method 8015M, December 1996

METHOD: $\quad$ EPA 8015M

ORDER ID: L0108129

MATRIX: Solid

ANALYST: SKV - Las Vegas Division

\begin{tabular}{|c|c|c|c|c|c|c|c|}
\hline $\begin{array}{l}\text { CLIENT } \\
\text { SAMPLE ID } \\
\end{array}$ & $\begin{array}{c}\text { SAMPLE } \\
\text { DATE }\end{array}$ & $\begin{array}{c}\text { NEL } \\
\text { SAMPLE ID } \\
\end{array}$ & $\begin{array}{c}\text { RESULT } \\
\mathrm{mg} / \mathrm{kg}\end{array}$ & C.R. & $\begin{array}{l}\text { Reporting } \\
\text { Limit }\end{array}$ & $\begin{array}{l}\text { Surrogate } \\
\text { Recovery* EXTRACTED }\end{array}$ & ANALYZED \\
\hline 029911-0-A & $8 / 10 / 01$ & L0108129-01 & $\mathrm{ND}$ & $\overline{\mathrm{ND}}$ & 20. $\mathrm{mg} / \mathrm{kg}$ & $8 / 17 / 01$ & $8 / 21 / 01$ \\
\hline
\end{tabular}

\section{C.R.: Carbon Range}

QUALITY CONTROL DATA (Total for Diesel Range):

\section{Sample ID}

Blank, 010817TP -BLK

LCS, 010817TPHS-1-LCS

LCSD, 010817TPHS-1-LCSD

MS, 010817TPHS-1-MS

MSD, 010817TPHS-1-MSD
Result

ND $<20 \mathrm{mg} / \mathrm{kg}$

$\begin{array}{lllll}60 & \% & 54 & -91 & \%\end{array}$

$69 \% \quad 54 \quad-.93 \quad \%$

$56 \% \quad 34-110 \%$

$55 \% \quad 34-110 \%$

* Surrogate used was Octacosane, acceptance limits 55-130\%.

ND - Not Detected

This report shall not be reproduced except in full, without the written approval of the laboratory. 
NEL LABORATORIES

CLIENT: Bechtel Nevada

PROJECT ID: V1236

PROJECT \#: 23081

TEST: $\quad$ Total Extractable Petroleum Hydrocarbons Fuel Finger Print by EPA Method 8015M, July 1992

\section{- $\quad$ MATRIX: Solid}

$\therefore \quad$ PARAMETER

Diesel Range (C12-C22)

Diesel Range (C12-C22)

Diesel Range (C12-C22)

Diesel Range (C12-C22)

Total

Total

Total

Total
NEL Sample W

010817TPHS-FP-LCS

010817TPHS-FP-LCSD

L0108129-01-MS

L0108129-01-MSD

010817TPHS-FP-LCS

010817TPHS-FP-LCSD

L0108129-01-MS

L0108129-01-MSD

\begin{tabular}{|c|c|}
\hline$\underset{\text { Spike }}{\text { Smount }}$ & $\frac{\text { Spike }}{\text { Result }}$ \\
\hline 166.7 & 105 \\
\hline 166.7 & 97 \\
\hline 166.7 & 93 \\
\hline 166.7 & 91 \\
\hline 166.7 & 105 \\
\hline 166.7 & 97 \\
\hline 166.7 & 93 \\
\hline 166.7 & 9 \\
\hline
\end{tabular}

Percent

Recovery

63

58

56

55

63

58

56

55
Acceptable

Range RPD

$53-91$

$53-91$

7.9

$34-114$

$34-114 \quad 2.2$

$53-91$

$53-91$

7.9

$34-114$

$34-114 \quad 2.2$ 


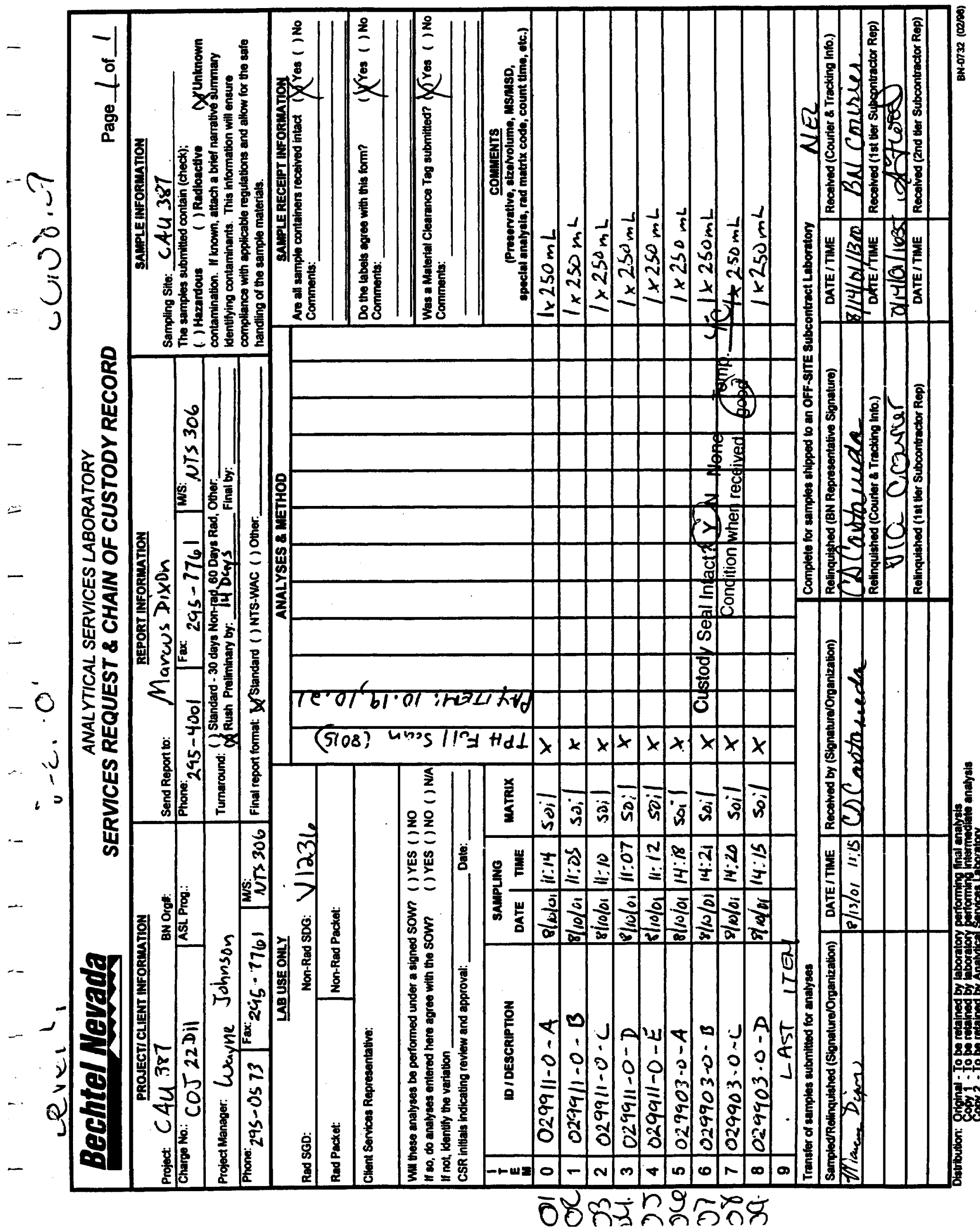


CLIENT:

Bechtel Nevada

P.O. Box $98521, \mathrm{M} / \mathrm{S}$ NTS273

Las Vegas, NV 89193-8521

$\cdots \quad$ ATTN:

Ted Redding

PROJECT NAME: $\quad$ V1249

NEL ORDER ID: L0109029

PROJECT NUMBER: 23081

Attached are the analytical results for samples in support of the above referenced project.

Samples submitted for this project were not sampled by NEL Laboratories. Samples were received by NEL in good condition, under chain of custody on 9/5/01.

Should you have any questions or comments, please feel free to contact our Client Services department at (702) $657-1010$.

\section{Some results have been flagged as follows:}

J - This concentration should be considered an estimate due laboratory control sample failure.

Some QA results have been flagged as follows:

J - This concentration should be considered an estimate due laboratory control sample failure.

J1 - The batch MS and/or MSD were outside acceptance limits. The batch LCS was acceptable.

R5 - RPD exceeded the laboratory control limit. Recovery met acceptance criteria.
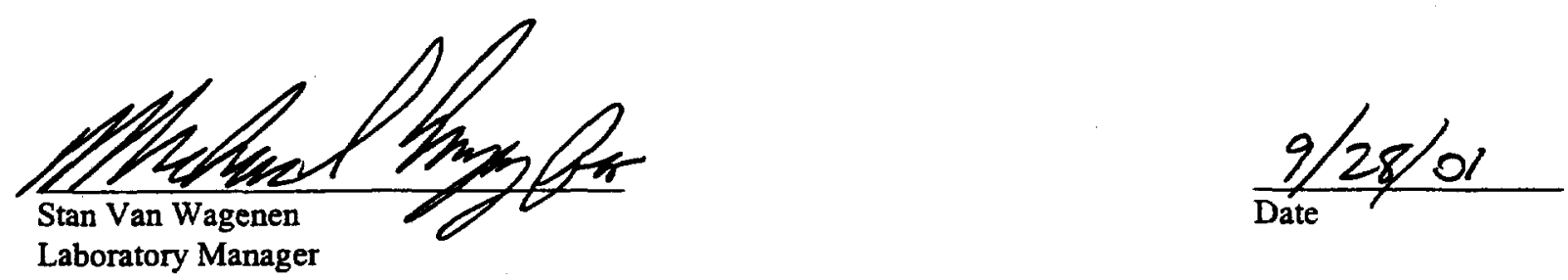

\section{CERTIFICATIONS:}

\begin{tabular}{|c|c|c|c|c|c|c|c|}
\hline & Reno & Las Vegas & S. California & & Reno & Las Vegas & S. California \\
\hline Arizona & AZ0520 & AZ0518 & AZ0605 & Idaho & Certified & Certified & \\
\hline California & 1707 & 2002 & 2264 & Montana & Certified & Certified & \\
\hline $\begin{array}{l}\text { US Army Corps } \\
\text { of Engineers }\end{array}$ & Certified & Certified & & $\begin{array}{l}\text { Nevada } \\
\text { L.A.C.S.D. }\end{array}$ & NV033 & NV052 & $\begin{array}{l}\text { CA084 } \\
10228\end{array}$ \\
\hline
\end{tabular}




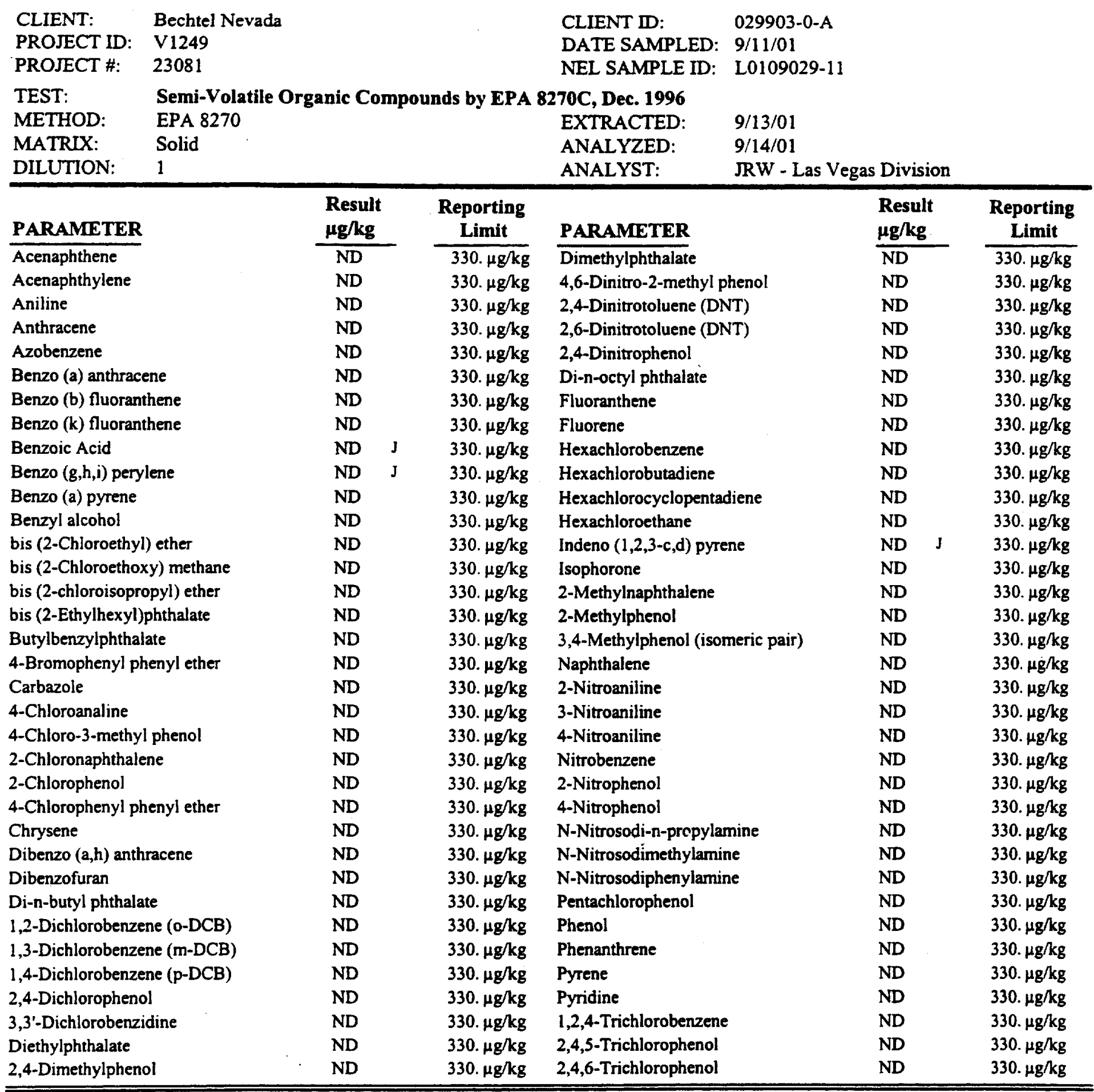

QUALITY CONTROL DATA:

\section{Surrogate}

2,4,6-Tribromophenol

2-Fluorobipheny]

2-Fluorophenol

Nitrobenzene-d5

$\mathrm{D}$-Terphenyl-d14

Phenol-d6
$\%$ Recovery

91

61

59

59

58

60
Acceptable Range

$19-122$

$30-115$

$25-121$

$23-120$

$18-137$

$24-113$

ND - Not Detected

This report shall not be reproduced except in full, without the written approval of the laboratory. 


$\begin{array}{llcl}\text { CLIENT: } & \text { Bechtel Nevada } & \text { CLIENT ID: } & \text { 029903-0-B } \\ \text { PROJECT ID: } & \text { V1249 } & \text { DATE SAMPLED: } & 9 / 11 / 01 \\ \text { PROJECT \#: } & 23081 & \text { NEL SAMPLE ID: } & \text { L0109029-12 } \\ \text { TEST: } & \text { Semi-Volatile Organic Compounds by EPA } & \text { 8270C, Dec. } 1996 & \\ \text { METHOD: } & \text { EPA 8270 } & \text { EXTRACTED: } & 9 / 13 / 01 \\ \text { MATRIX: } & \text { Solid } & \text { ANALYZED: } & 9 / 14 / 01 \\ \text { DILUTION: } & 1 & \text { ANALYST: } & \text { JRW - Las Vegas Division }\end{array}$

\begin{tabular}{|c|c|c|c|c|c|c|}
\hline PARAMETER & $\begin{array}{l}\text { Result } \\
\mu g / k g\end{array}$ & & $\begin{array}{c}\text { Reporting } \\
\text { Limit }\end{array}$ & PARAMETER & $\begin{array}{l}\text { Result } \\
\mu \mathrm{g} / \mathrm{kg}\end{array}$ & $\begin{array}{c}\text { Reporting } \\
\text { Limit }\end{array}$ \\
\hline Acenaphthene & $\mathrm{ND}$ & & $330 . \mu \mathrm{g} / \mathrm{kg}$ & $\overline{\text { Dimethylphthalate }}$ & ND & $330 . \mu \mathrm{g} / \mathrm{kg}$ \\
\hline Acenaphthylene & ND & & $330 . \mu g / k g$ & 4,6-Dinitro-2-methyl phenol & ND & 330. $\mu \mathrm{g} / \mathrm{kg}$ \\
\hline Aniline & ND & & $330 . \mu \mathrm{g} / \mathrm{kg}$ & 2,4-Dinitrotoluene (DNT) & ND & 330. $\mu \mathrm{g} / \mathrm{kg}$ \\
\hline Anthracene & ND & & $330 . \mu \mathrm{g} / \mathrm{kg}$ & 2,6-Dinitrotoluene (DNT) & ND & 330. $\mathrm{\mu g} / \mathrm{kg}$ \\
\hline Azobenzene & ND & & 330. $\mu \mathrm{g} / \mathrm{kg}$ & 2,4-Dinitrophenol & ND & 330. $\mu \mathrm{g} / \mathrm{kg}$ \\
\hline Benzo (a) anthracene & ND & & $330 . \mu \mathrm{g} / \mathrm{kg}$ & Di-n-octyl phthalate & ND & 330. $\mu \mathrm{g} / \mathrm{kg}$ \\
\hline Benzo (b) fluoranthene & ND & & $330 . \mu \mathrm{g} / \mathrm{kg}$ & Fluoranthene & ND & 330. $\mu \mathrm{g} / \mathrm{kg}$ \\
\hline Benzo (k) fluoranthene & ND & & 330. $\mu \mathrm{g} / \mathrm{kg}$ & Fluorene & ND & 330. $\mu \mathrm{g} / \mathrm{kg}$ \\
\hline Benzoic Acid & ND & $\mathbf{J}$ & 330. $\mu \mathrm{g} / \mathrm{kg}$ & Hexachlorobenzene & ND & 330. $\mu \mathrm{g} / \mathrm{kg}$ \\
\hline Benzo $(g, h, i)$ perylene & ND & $\mathbf{J}$ & 330. $\mu \mathrm{g} / \mathrm{kg}$ & Hexachlorobutadiene & ND & 330. $\mu \mathrm{g} / \mathrm{kg}$ \\
\hline Benzo (a) pyrene & ND & & 330. $\mu \mathrm{g} / \mathrm{kg}$ & Hexachlorocyclopentadiene & ND & $330 . \mu \mathrm{g} / \mathrm{kg}$ \\
\hline Benzyl alcohol & ND & & 330. $\mu \mathrm{g} / \mathrm{kg}$ & Hexachloroethane & ND & 330. $\mu \mathrm{g} / \mathrm{kg}$ \\
\hline bis (2-Chloroethyl) ether & ND & & $330 . \mu \mathrm{g} / \mathrm{kg}$ & Indeno $(1,2,3-c, d)$ pyrene & ND & $330 . \mu \mathrm{g} / \mathrm{kg}$ \\
\hline bis (2-Chloroethoxy) methane & ND & & 330. $\mu \mathrm{g} / \mathrm{kg}$ & Isophorone & ND & $330 . \mu \mathrm{g} / \mathrm{kg}$ \\
\hline bis (2-chloroisopropyl) ether & ND & & $330 . \mu \mathrm{g} / \mathrm{kg}$ & 2-Methylnaphthalene & ND & 330. $\mu \mathrm{g} / \mathrm{kg}$ \\
\hline bis (2-Ethylhexyl)phthalate & ND & & 330. $\mu \mathrm{g} / \mathrm{kg}$ & 2-Methylphenol & ND & $330 . \mu \mathrm{g} / \mathrm{kg}$ \\
\hline Butylbenzylphthalate & ND & & 330. $\mu \mathrm{g} / \mathrm{kg}$ & 3,4-Methylphenol (isomeric pair) & ND & $330 . \mu \mathrm{g} / \mathrm{kg}$ \\
\hline 4-Bromophenyl phenyl ether & ND & & $330 . \mu \mathrm{g} / \mathrm{kg}$ & Naphthalene & ND & $330 . \mu \mathrm{g} / \mathrm{kg}$ \\
\hline Carbazole & ND & & $330 . \mu \mathrm{g} / \mathrm{kg}$ & 2-Nitroaniline & ND & $330 . \mu \mathrm{g} / \mathrm{kg}$ \\
\hline 4-Chloroanaline & ND & & $330 . \mu g / k g$ & 3-Nitroaniline & ND & 330. $\mu \mathrm{g} / \mathrm{kg}$ \\
\hline 4-Chloro-3-methyl phenol & ND & & $330 . \mu \mathrm{g} / \mathrm{kg}$ & 4-Nitroaniline & ND & 330. $\mu \mathrm{g} / \mathrm{kg}$ \\
\hline 2-Chloronaphthalene & ND & & $330 . \mu \mathrm{g} / \mathrm{kg}$ & Nitrobenzene & ND & $330 . \mu \mathrm{g} / \mathrm{kg}$ \\
\hline 2-Chlorophenol & ND & & $330 . \mu \mathrm{g} / \mathrm{kg}$ & 2-Nitrophenol & ND & 330. $\mu \mathrm{g} / \mathrm{kg}$ \\
\hline 4-Chlorophenyl phenyl ether & $\mathrm{ND}$ & & $330 . \mu \mathrm{g} / \mathrm{kg}$ & 4-Nitrophenol & ND & 330. $\mu \mathrm{g} / \mathrm{kg}$ \\
\hline Chrysene & ND & & $330 . \mu \mathrm{g} / \mathrm{kg}$ & N-Nitrosodi-n-propylamine & ND & 330. $\mu \mathrm{g} / \mathrm{kg}$ \\
\hline Dibenzo $(a, h)$ anthracene. & ND & & $330 . \mu \mathrm{g} / \mathbf{k g}$ & N-Nitrosodimethylamine & ND & 330. $\mu \mathrm{g} / \mathrm{kg}$ \\
\hline Dibenzofuran & ND & & $330 . \mu \mathrm{g} / \mathrm{kg}$ & N-Nitrosodiphenylamine & $\mathrm{ND}$ & 330. $\mu \mathrm{g} / \mathrm{kg}$ \\
\hline Di-n-butyl phthalate & ND & & $330 . \mu \mathrm{g} / \mathrm{kg}$ & Pentachlorophenol & ND & 330. $\mu \mathrm{g} / \mathrm{kg}$ \\
\hline 1,2-Dichlorobenzene (o-DCB) & ND & & $330 . \mu \mathrm{g} / \mathrm{kg}$ & Phenol & ND & 330. $\mu \mathrm{g} / \mathrm{kg}$ \\
\hline 1,3-Dichlorobenzene (m-DCB) & ND & & 330. $\mu \mathrm{g} / \mathrm{kg}$ & Phenanthrene & ND & $330 . \mu \mathrm{g} / \mathrm{kg}$ \\
\hline 1,4-Dichlorobenzene (p-DCB) & ND & & 330. $\mu \mathrm{g} / \mathrm{kg}$ & Pyrene & ND & 330. $\mu \mathrm{g} / \mathrm{kg}$ \\
\hline 2,4-Dichlorophenol & ND & & 330. $\mu \mathrm{g} / \mathrm{kg}$ & Pyridine & ND & $330 . \mu \mathrm{g} / \mathrm{kg}$ \\
\hline 3,3'-Dichlorobenzidine & ND & & $330 . \mu \mathrm{g} / \mathrm{kg}$ & 1,2,4-Trichlorobenzene & ND & $330 . \mu \mathrm{g} / \mathrm{kg}$ \\
\hline Diethylphthalate & ND & & 330. $\mu \mathrm{g} / \mathrm{kg}$ & 2,4,5-Trichlorophenol & ND & $330 . \mu \mathrm{g} / \mathrm{kg}$ \\
\hline 2,4-Dimethylphenol & ND & & $330 . \mu \mathrm{g} / \mathrm{kg}$ & 2,4,6-Trichlorophenol & ND & 330. $\mu \mathrm{g} / \mathrm{kg}$ \\
\hline
\end{tabular}

QUALITY CONTROL DATA:

\section{Surrogate}

2,4,6-Tribromophenol

2-Fluorobiphenyl

2-Fluorophenol

Nitrobenzene-d5

p-Terphenyl-d14

Phenol-d6

\section{$\%$ Recovery}

87

57

54

53

75

58

\section{Acceptable Range}

$19-122$

$30-115$

$25-121$

$23-120$

$18-137$

$24-113$

ND - Not Detected

This report shall not be reproduced except in full, without the written approval of the laboratory. 


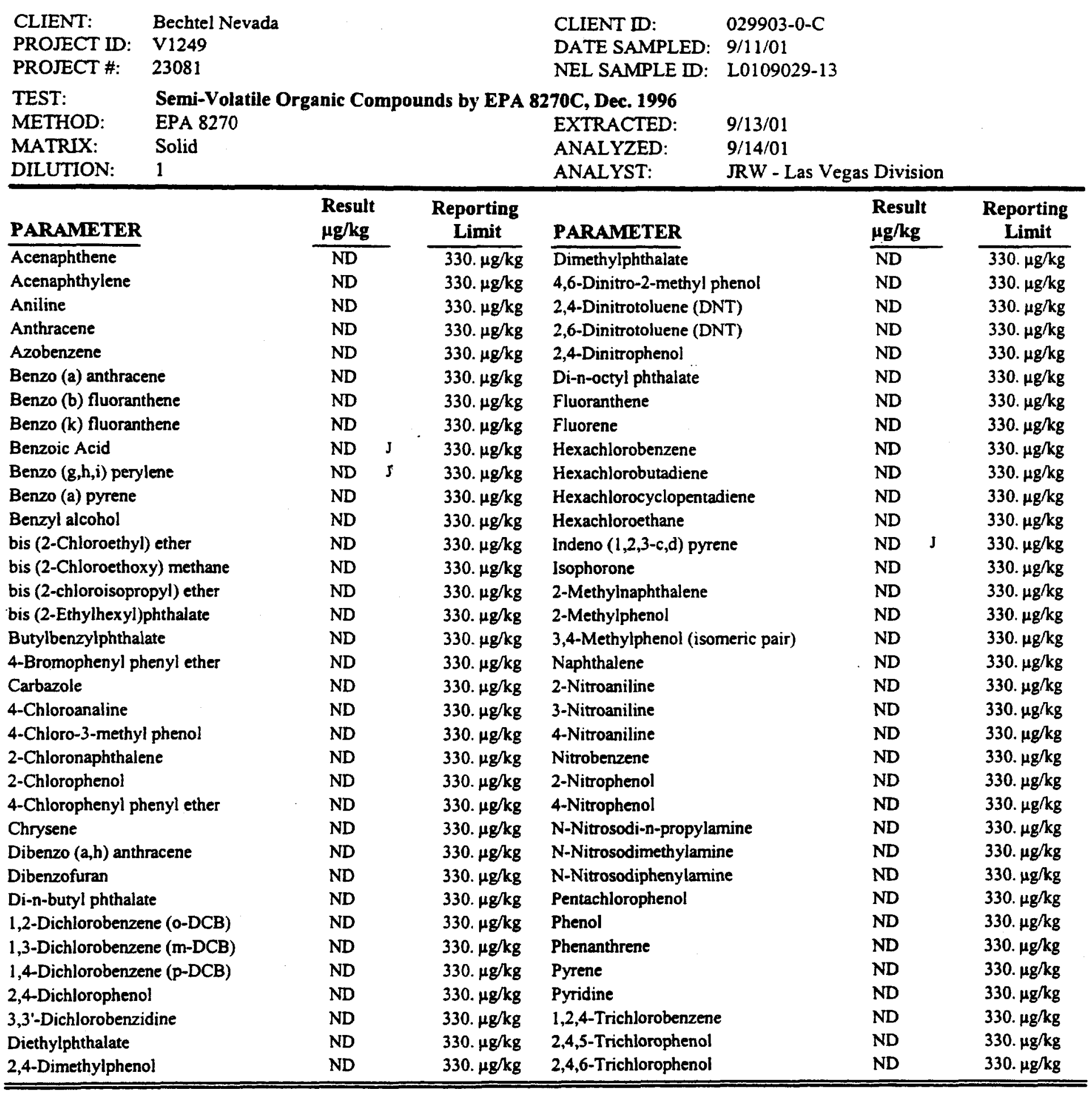

QUALITY CONTROL DATA:

Surrogate

2,4,6-Tribromophenol

2-Fluorobiphenyl

2-Fluorophenol

Nitrobenzene-d5

p-Terphenyl-d14

Phenol-d6
$\%$ Recovery

79

66

62

60

72

64
Acceptable Range

$19-122$

$30-115$

$25-121$

$23-120$

$18-137$

$24-113$

ND - Not Detected

This report shall not be reproduced except in full, without the written approval of the laboratory. 


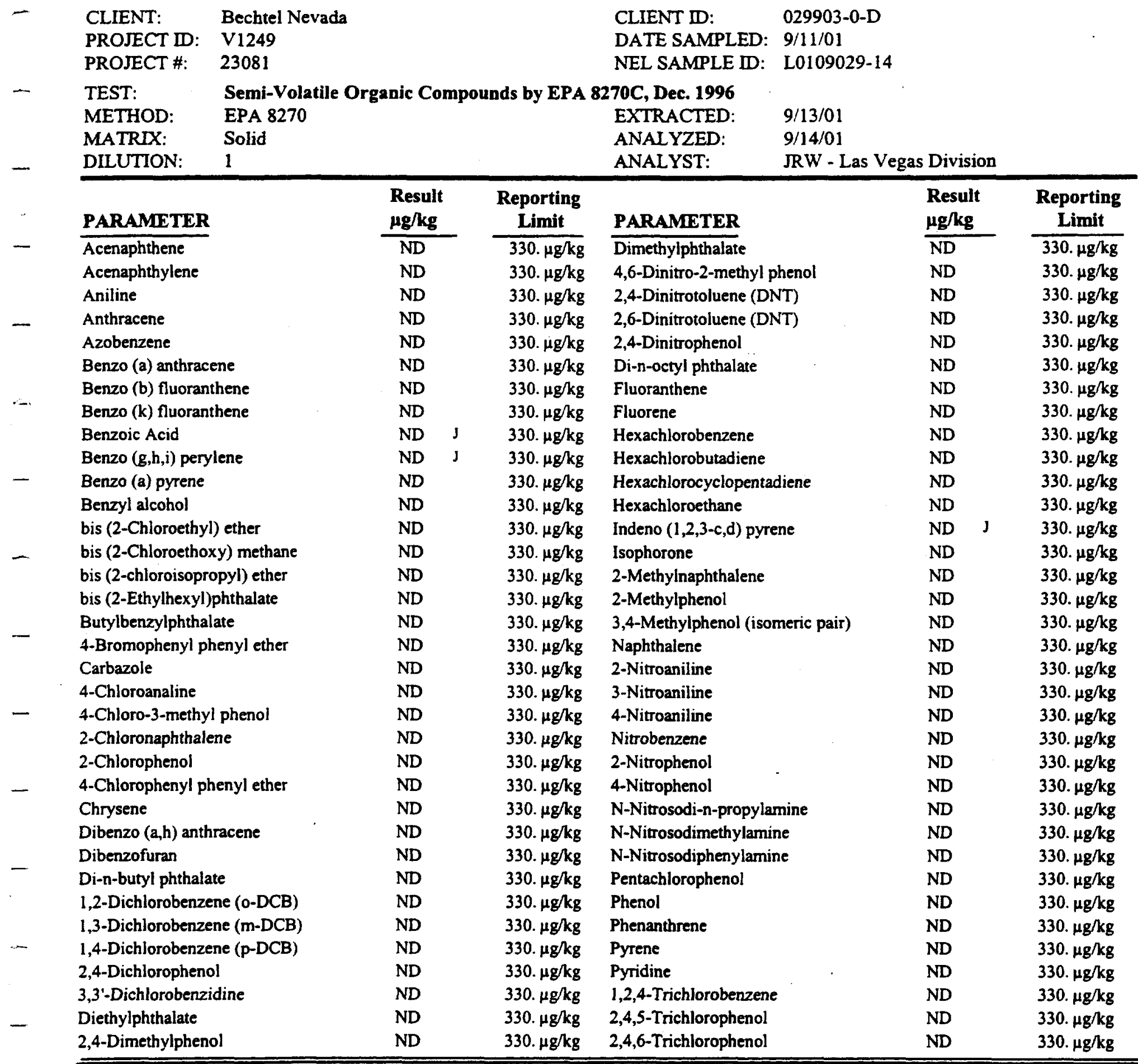

QUALITY CONTROL DATA:

Surrogate

2,4,6-Tribromophenol

2-Fluorobiphenyl

2-Fluorophenol

Nitrobenzene-d5

p-Terphenyl-d14

Phenol-d6
$\%$ Recovery

80

59

54

51

74

58
Acceptable Range

$19-122$

$30-115$

$25-121$

$23-120$

$18-137$

$24-113$

ND - Not Detected

This report shall not be reproduced except in full, without the written approval of the laboratory. 


$\begin{array}{llcl}\text { CLIENT: } & \text { Bechtel Nevada } & \text { CLIENT ID: } & \text { Method Blank } \\ \text { PROJECT DD: } & \text { V1249 } & \text { DATE SAMPLED: } & \text { NA } \\ \text { PROJECT \#: } & 23081 & \text { NEL SAMPLE ID: } & \text { 010913-8270S1-BLK } \\ \text { TEST: } & \text { Semi-Volatile Organic Compounds by EPA } & \text { 8270C, Dec. 1996 } & \\ \text { METHOD: } & \text { EPA } 8270 & \text { ANALYST: } & \text { JRW - Las Vegas Division } \\ \text { MATRIX: } & \text { Solid } & \text { EXTRACTED: } & 9 / 13 / 01 \\ & & \text { ANALYZED: } & 9 / 14 / 01\end{array}$

\begin{tabular}{|c|c|c|c|c|c|}
\hline PARAMETER & $\begin{array}{l}\text { Result } \\
\mu \mathrm{g} / \mathrm{kg}\end{array}$ & $\begin{array}{l}\text { Reporting } \\
\text { Limit }\end{array}$ & PARAMETER & $\begin{array}{l}\text { Result } \\
\mu \mathrm{g} / \mathrm{kg}\end{array}$ & $\begin{array}{l}\text { Reporting } \\
\text { Limit }\end{array}$ \\
\hline Acenaphthene & ND & $330 \mu \mathrm{g} / \mathrm{kg}$ & Diethylphthalate & ND & $330 \mu \mathrm{g} / \mathrm{kg}$ \\
\hline Acenaphthylene & ND & $330 \mu \mathrm{g} / \mathrm{kg}$ & 2,4-Dimethylphenol & ND & $330 \mu \mathrm{g} / \mathrm{kg}$ \\
\hline Aniline & $\mathrm{ND}$ & $330 \mu \mathrm{g} / \mathrm{kg}$ & Dimethy!phthalate & ND & $330 \mu \mathrm{g} / \mathrm{kg}$ \\
\hline Anthracene & ND & $330 \mu \mathrm{g} / \mathrm{kg}$ & 4,6-Dinitro-2-methyl phenol & ND & $330 \mu \mathrm{g} / \mathrm{kg}$ \\
\hline Azobenzene & ND & $330 \mu \mathrm{g} / \mathrm{kg}$ & 2,4-Dinitrotoluene (DNT) & ND & $330 \mu \mathrm{g} / \mathrm{kg}$ \\
\hline Benzo (a) anthracene & ND & $330 \mu \mathrm{g} / \mathrm{kg}$ & 2,6-Dinitrotoluene (DNT) & ND & $330 \mu \mathrm{g} / \mathrm{kg}$ \\
\hline Benzo (b) fluoranthene & ND & $330 \mu \mathrm{g} / \mathrm{kg}$ & 2,4-Dinitrophenol & ND & $330 \mu \mathrm{g} / \mathrm{kg}$ \\
\hline Benzo (k) fluoranthene & ND & $330 \mu \mathrm{g} / \mathrm{kg}$ & Di-n-octyl phthalate & ND & $330 \mu \mathrm{g} / \mathrm{kg}$ \\
\hline Benzoic Acid & ND & $330 \mu \mathrm{g} / \mathrm{kg}$ & Fluoranthene & ND & $330 \mu \mathrm{g} / \mathrm{kg}$ \\
\hline Benzo $(\mathrm{g}, \mathrm{h}, \mathrm{i})$ perylene & ND & $330 \mu \mathrm{g} / \mathrm{kg}$ & Fluorene & ND & $330 \mu \mathrm{g} / \mathrm{kg}$ \\
\hline Benzo (a) pyrene & ND & $330 \mu \mathrm{g} / \mathrm{kg}$ & Hexachlorobenzene & ND & $330 \mu \mathrm{g} / \mathrm{kg}$ \\
\hline Benzyl alcohol & ND & $330 \mu \mathrm{g} / \mathrm{kg}$ & Hexachlorobutadiene & ND & $330 \mu \mathrm{g} / \mathrm{kg}$ \\
\hline bis (2-Chloroethyl) ether & ND & $330 \mu \mathrm{g} / \mathrm{kg}$ & Hexachlorocyclopentadiene & ND & $330 \mu \mathrm{g} / \mathrm{kg}$ \\
\hline bis (2-Chloroethoxy) methane & ND & $330 \mu \mathrm{g} / \mathrm{kg}$ & Hexachloroethane & ND & $330 \mu \mathrm{g} / \mathrm{kg}$ \\
\hline bis (2-chloroisopropyl) ether & ND & $330 \mu \mathrm{g} / \mathrm{kg}$ & Indeno $(1,2,3-c, d)$ pyrene & ND & $330 \mu \mathrm{g} / \mathrm{kg}$ \\
\hline bis (2-Ethylhexyl)phthalate & ND & $330 \mu \mathrm{g} / \mathrm{kg}$ & Isophorone & ND & $330 \mu \mathrm{g} / \mathrm{kg}$ \\
\hline Butylbenzylphthalate & ND & $330 \mu \mathrm{g} / \mathrm{kg}$ & 2-Methylnaphthalene & ND & $330 \mu \mathrm{g} / \mathrm{kg}$ \\
\hline 4-Bromophenyl phenyl ether & ND & $330 \mu \mathrm{g} / \mathrm{kg}$ & 2-Methylphenol & ND & $330 \mu \mathrm{g} / \mathrm{kg}$ \\
\hline Carbazole & ND & $330 \mu \mathrm{g} / \mathrm{kg}$ & 3,4-Methylphenol (isomeric pair) & ND & $330 \mu \mathrm{g} / \mathrm{kg}$ \\
\hline 4-Chloroanaline & ND & $330 \mu \mathrm{g} / \mathrm{kg}$ & Naphthalene & ND & $330 \mu \mathrm{g} / \mathrm{kg}$ \\
\hline 4-Chloro-3-methyl phenol & ND & $330 \mu \mathrm{g} / \mathrm{kg}$ & 2-Nitroaniline & ND & $330 \mu \mathrm{g} / \mathrm{kg}$ \\
\hline 2-Chloronaphthalene & ND & $330 \mu \mathrm{g} / \mathrm{kg}$ & 3-Nitroaniline & ND & $330 \mu \mathrm{g} / \mathrm{kg}$ \\
\hline 2-Chlorophenol & ND & $330 \mu \mathrm{g} / \mathrm{kg}$ & 4-Nitroaniline & ND & $330 \mu \mathrm{g} / \mathrm{kg}$ \\
\hline 4-Chlorophenyl phenyl ether & ND & $330 \mu \mathrm{g} / \mathrm{kg}$ & Nitrobenzene & ND & $330 \mu \mathrm{g} / \mathrm{kg}$ \\
\hline Chrysene & ND & $330 \mu \mathrm{g} / \mathrm{kg}$ & 2-Nitrophenol & ND & $330 \mu \mathrm{g} / \mathrm{kg}$ \\
\hline Dibenzo $(\mathrm{a}, \mathrm{h})$ anthracene & ND & $330 \mu \mathrm{g} / \mathrm{kg}$ & 4-Nitrophenol & ND & $330 \mu \mathrm{g} / \mathrm{kg}$ \\
\hline Dibenzofuran & ND & $330 \mu \mathrm{g} / \mathrm{kg}$ & N-Nitrosodi-n-propylamine & ND & $330 \mu \mathrm{g} / \mathrm{kg}$ \\
\hline Di-n-butyl phthalate & ND & $330 \mu \mathrm{g} / \mathrm{kg}$ & N-Nitrosodimethylamine & ND & $330 \mu \mathrm{g} / \mathrm{kg}$ \\
\hline 1,2-Dichlorobenzene (o-DCB) & ND & 330 Hg/kg & N-Nitrosodiphenylamine & ND & $330 \mu \mathrm{g} / \mathrm{kg}$ \\
\hline 1,3-Dichlorobenzene (m-DCB) & ND & 330 Hg/kg & Pentachlorophenol & ND & $330 \mu \mathrm{g} / \mathrm{kg}$ \\
\hline 1,4-Dichlorobenzene ( $p$-DCB) & ND & $330 \mu g / k g$ & Phenol & ND & $330 \mu \mathrm{g} / \mathrm{kg}$ \\
\hline 2,4-Dichlorophenol & ND & $330 \mu \mathrm{g} / \mathrm{kg}$ & Phenanthrene & ND & $330 \mu \mathrm{g} / \mathrm{kg}$ \\
\hline 3,3'-Dichlorobenzidine & ND & $330 \mu \mathrm{g} / \mathrm{kg}$ & Pyrene & ND & $330 \mu \mathrm{g} / \mathrm{kg}$ \\
\hline
\end{tabular}

QUALITY CONTROL DATA:

Surrogate
2,4,6-Tribromophenol
2-Fluorobiphenyl
Nitrobenzene-d5
p-Terphenyl-d14
Phenol-d6

\begin{tabular}{c} 
\% Recovery \\
\hline 57 \\
62 \\
57 \\
63 \\
69 \\
62
\end{tabular}

\begin{tabular}{l} 
Acceptable Range \\
\hline $19-122$ \\
$30-115$ \\
$25-121$ \\
$23-120$ \\
$18-137$ \\
$24-113$
\end{tabular}

ND - Not Detected

This report shall not be reproduced except in full, without the written approval of the laboratory. 
NEL LABORATORIES

\begin{tabular}{|c|c|c|}
\hline $\begin{array}{l}\text { CLIENT: } \\
\text { PROJECT ID: } \\
\text { PROJECT \#: }\end{array}$ & $\begin{array}{l}\text { Bechtel Nevada } \\
\text { V1249 } \\
23081\end{array}$ & $\begin{array}{ll}\text { CLIENT ID: } & \text { 039908-0-A } \\
\text { DATE SAMPLED: } & 9 / 4 / 01 \\
\text { NEL SAMPLE ID: } & \text { L0109029-06 }\end{array}$ \\
\hline
\end{tabular}

\section{- TEST: \\ METHOD: EPA 8015M}

Total Extractable Petroleum Hydrocarbons Fuel Finger Print by EPA Method 8015M, July 1992 MATRIX:

DILUTION: Solid

\section{ANAIYST:}

EXTRACTED:

ANALYZED:

PXC - Division

1

PARAMETER

Gasoline Range (C8-C12)

Diesel Range (C12-C22)

Oil Range (C22-C34)

Total

QUALITY CONTROL DATA:

Surrogate

Octacosane

ANALYZED: $\quad 9 / 13 / 01$

\begin{tabular}{l} 
Result \\
\hline ND \\
ND \\
ND \\
ND \\
\hline
\end{tabular}

\% Recovery

86

\section{Reporting}

Limit

10. $\mathrm{mg} / \mathrm{kg}$

10. $\mathrm{mg} / \mathrm{kg}$

50. $\mathrm{mg} / \mathrm{kg}$

10. $\mathrm{mg} / \mathrm{kg}$ $x-$ Octacosane

ND - Not Detected

This report shall not be reproduced except in full, without the written approval of the laboratory.
Acceptable Range

$54-130$ 
NEL LABORATORIES

\begin{tabular}{|c|c|c|}
\hline $\begin{array}{l}\text { CLIENT: } \\
\text { PROJECT ID: }\end{array}$ & $\begin{array}{l}\text { Bechtel Nevada } \\
\text { V1249 } \\
23081\end{array}$ & $\begin{array}{ll}\text { CLIENT I: } & \text { 039908-0-B } \\
\text { DATE SAMPLED: } & 9 / 4 / 01 \\
\text { NEL SAMPLE ID: } & \text { L } 0109029-07\end{array}$ \\
\hline
\end{tabular}

TEST: Total Extractable Petroleum Hydrocarbons Fuel Finger Print by EPA Method 8015M, July 1992

METHOD: EPA 8015M ANALYST: PXC - Division

MATRIX: Solid $\quad$ EXTRACTED: 9/12/01

DILUTION: $1 \quad$ ANALYZED: $\quad 9 / 13 / 01$

\begin{tabular}{lcc}
\hline PARAMETER & Result & Reporting \\
\cline { 2 - 3 } & $\mathrm{ND}$ & $\frac{\text { Limit }}{10 . \mathrm{mg} / \mathrm{kg}}$ \\
Gasoline Range $(\mathrm{C} 8-\mathrm{Cl2})$ & $\mathrm{ND}$ & $10 . \mathrm{mg} / \mathrm{kg}$ \\
Diesel Range $(\mathrm{C} 12-\mathrm{C22})$ & $\mathrm{ND}$ & $50 . \mathrm{mg} / \mathrm{kg}$ \\
Oil Range (C22-C34) & $\mathrm{ND}$ & $10 . \mathrm{mg} / \mathrm{kg}$ \\
\hline Total & \\
\hline \hline
\end{tabular}

QUALITY CONTROL DATA:

Surrogate

\% Recovery

Octacosane

73

Acceptable Range

$54-130$

ND - Not Detected

This report shall not be reproduced except in full, without the written approval of the laboratory. 
NEL LABORATORIES

\begin{tabular}{lll}
\hline CLIENT: & Bechtel Nevada & CLIENT ID: \\
PROJECT ID: & V1249 & DATE SAMPLE-0-C \\
PROJECT \#: & 23081 & NEL SAMPLE ID: L0109029-08
\end{tabular}

- TEST:

METHOD:

MATRIX:

DILUTION:
Total Extractable Petroleum Hydrocarbons Fuel Finger Print by EPA Method 8015M, July 1992 EPA $8015 \mathrm{M}$

Solid

1
ANALYST:

EXTRACTED

ANALYZED:
PXC - Division

9/12/01

$9 / 13 / 01$

PARAMETER

Gasoline Range ( $\mathrm{C} 8-\mathrm{Cl} 2)$

Diesel Range (C12-C22)

Oil Range (C22-C34)

Total

\begin{tabular}{rl}
\multicolumn{2}{c}{ Result } \\
\cline { 1 - 1 } $\mathrm{ND}$ & \\
22 & $\mathrm{mg} / \mathrm{kg}$ \\
$\mathrm{ND}$ & \\
22 & $\mathrm{mg} / \mathrm{kg}$ \\
\hline
\end{tabular}

QUALITY CONTROL DATA:

Surrogate

.. Octacosane

$\%$ Recovery

105

Acceptable Range

- ND - Not Detected

This report shall not be reproduced except in full, without the written approval of the laboratory.

Reporting

Limit

10. $\mathrm{mg} / \mathrm{kg}$

10. $\mathrm{mg} / \mathrm{kg}$

50. $\mathrm{mg} / \mathrm{kg}$

10. $\mathrm{mg} / \mathrm{kg}$

$54-130$ 
NEL LABORATORIES

\begin{tabular}{|c|c|c|}
\hline $\begin{array}{l}\text { CLIENT: } \\
\text { PROJECT ID: }\end{array}$ & $\begin{array}{l}\text { Bechtel Nevada } \\
\text { V1249 }\end{array}$ & $\begin{array}{ll}\text { CLIENT D: } & \text { 039908-0-D } \\
\text { DATE SAMPLED: } & \mathbf{9} / 4 / 01\end{array}$ \\
\hline PROJECT \#: & 23081 & NEL SAMPLE ID: L0109029-09 \\
\hline
\end{tabular}

TEST: $\quad$ Total Extractable Petroleum Hydrocarbons Fuel Finger Print by EPA Method 8015M, July 1992

METHOD: EPA 8015M ANALYST: PXC - Division

MATRIX: Solid $\quad$ EXTRACTED: $9 / 12 / 01$

DILUTION: $1 \quad$ ANALYZED: $\quad 9 / 13 / 01$

\begin{tabular}{llc}
\hline PARAMETER & Result & Reporting \\
\cline { 2 - 3 } & ND & $\frac{\text { Limit }}{10 . \mathrm{mg} / \mathrm{kg}}$ \\
Gasoline Range (C8-C12) & $\mathrm{ND}$ & $10 . \mathrm{mg} / \mathrm{kg}$ \\
Diesel Range (C12-C22) & ND & $50 . \mathrm{mg} / \mathrm{kg}$ \\
Oil Range (C22-C34) & $\mathrm{ND}$ & $10 . \mathrm{mg} / \mathrm{kg}$ \\
\hline Total & \\
\hline \hline
\end{tabular}

QUALITY CONTROL DATA:

Surrogate

\% Recovery

87

Octacosane

ND - Not Detected

This report shall not be reproduced except in full, without the written approval of the laboratory.
Acceptable Range

$54-130$ 
NEL LABORATORIES

\begin{tabular}{lll}
\hline CLIENT: & Bechtel Nevada & CLIENT ID: 0 039906-0-A \\
PROJECT ID: & V1249 & DATE SAMPLED: $9 / 4 / 01$ \\
PROJECT \#: & 23081 & NEL SAMPLE ID: L0109029-10
\end{tabular}

- TEST: $\quad$ Total Extractable Petroleum Hydrocarbons Fuel Finger Print by EPA Method 8015 M, July 1992

METHOD: EPA 8015M

MATRIX: Solid

ANALYST: $\quad$ PXC - Division

DILUTION: 1

EXTRACTED: $\quad 9 / 12 / 01$

ANALYZED: $\quad 9 / 13 / 01$

PARAMETER

Gasoline Range (C8-C12)

Diesel Range (C12-C22)

Oil Range (C22-C34)

Total

QUALITY CONTROL DATA:

Surrogate

Octacosane
$\%$ Recovery

89

\section{Reporting}

Limit

10. $\mathrm{mg} / \mathrm{kg}$

10. $\mathrm{mg} / \mathrm{kg}$

50. $\mathrm{mg} / \mathrm{kg}$

10. $\mathrm{mg} / \mathrm{kg}$

$25 \mathrm{mg} / \mathrm{kg}$

Acceptable Range

$54-130$

ND - Not Detected

This report shall not be reproduced except in full, without the written approval of the laboratory. 


\section{NEL LABORATORIES}

$\begin{array}{llll}\text { CLIENT: } & \text { Bechtel Nevada } & \text { CLIENT D: } & \text { Method Blank } \\ \text { PROJECT ID: } & \text { V1249 } & \text { DATE SAMPLED: NA } \\ \text { PROJECT \#: } & 23081 & \text { NEL SAMPLE ID: } 010912 \text { TPHS-BLK }\end{array}$

TEST: $\quad$ Total Extractable Petroleum Hydrocarbons Fuel Finger Print by EPA Method 8015M, July 1992

METHOD: EPA 8015M ANALYST: PXC - Division

MATRIX: Solid $\quad$ EXTRACTED: $9 / 12 / 01$

ANALYZED: $\quad 9 / 13 / 01$

\begin{tabular}{llc}
\hline PARAMETER & Result & Reporting \\
\cline { 2 - 3 } & $\mathrm{ND}$ & $\frac{\text { Limit }}{10 . \mathrm{mg} / \mathrm{kg}}$ \\
Gasoline Range (C8-C12) & $\mathrm{ND}$ & $10 . \mathrm{mg} / \mathrm{kg}$ \\
Diesel Range (C12-C22) & $\mathrm{ND}$ & $50 . \mathrm{mg} / \mathrm{kg}$ \\
Oil Range (C22-C34) & $\mathrm{ND} \mathrm{B}$ & $10 . \mathrm{mg} / \mathrm{kg}$ \\
\hline Total & \\
\hline
\end{tabular}

QUALITY CONTROL DATA:

Surrogate

Octacosane

\% Recovery

87

ND - Not Detected

This report shall not be reproduced except in full, without the written approval of the laboratory.

\section{Acceptable Range}

$54-130$ 


\begin{tabular}{|c|c|c|c|c|c|c|c|}
\hline \multirow{2}{*}{$\begin{array}{l}\text { CLIENT: } \\
\text { PROJECT ID: } \\
\text { PROJECT \#: } \\
\text { TEST: } \\
\text { MATRI: }\end{array}$} & \multicolumn{7}{|c|}{$\begin{array}{l}\text { Bechtel Nevada } \\
\text { V1249 } \\
23081\end{array}$} \\
\hline & \multicolumn{7}{|c|}{$\begin{array}{l}\text { Total Extractable Petroleum Hydrocarbons Fuel Finger Print by EPA Method 8015M, July } 1992 \\
\text { Solid }\end{array}$} \\
\hline \multicolumn{2}{|l|}{ PARAMETER } & NEL Sample ID & $\begin{array}{c}\text { Spike } \\
\text { Amount }\end{array}$ & $\begin{array}{l}\text { Spike } \\
\text { Result }\end{array}$ & $\frac{\text { Percent }}{\text { Recovery }}$ & $\frac{\text { Acceptable }}{\underline{\text { Range }}}$ & $\underline{\mathbf{R P D}}$ \\
\hline \multicolumn{2}{|c|}{ Diesel Range (C12-C22) } & 010912TPHS-LCS & 166.7 & 120 & 72 & $53-91$ & \\
\hline \multicolumn{2}{|c|}{ Diesel Range (C12-C22) } & 010912TPHS-LCSD & 166.7 & 110 & 66 & $53-91$ & 8.7 \\
\hline \multicolumn{2}{|c|}{ Diesel Range (C12-C22) } & L0109029-03-MS & 166.7 & 133 & 80 & $34-114$ & \\
\hline \multicolumn{2}{|c|}{ Diesel Range (C12-C22) } & L0109029-03-MSD & 166.7 & 45 & 27 & $34-114$ & 98.9 \\
\hline \multicolumn{2}{|l|}{ Total } & 010912 TPHS-LCS & 166.7 & 120 & 72 & $53-91$ & \\
\hline \multicolumn{2}{|l|}{ Total } & 010912 TPHS-LCSD & 166.7 & 110 & 66 & $53-91$ & 8.7 \\
\hline \multicolumn{2}{|l|}{ Total } & L0109029-03-MS & 166.7 & 133 & 80 & $34-114$ & \\
\hline \multicolumn{2}{|l|}{ Total } & L0109029-03-MSD & 166.7 & 45 & 27 & $34-114$ & 98.9 \\
\hline
\end{tabular}




\author{
CLIENT: Bechtel Nevada \\ PROJECT ID: V1249 \\ PROJECT \#: 23081 \\ TEST: $\quad$ Semi-Volatile Organic Compounds by EPA 8270C, Dec. 1996 \\ MATRIX: $\quad$ Solid
}

\begin{tabular}{|c|c|c|c|c|c|c|c|}
\hline PARAMETER & NEL Sample ID & $\begin{array}{c}\text { Spike } \\
\text { Amount }\end{array}$ & $\frac{\text { Spike }}{\underline{\text { Result }}}$ & $\begin{array}{l}\text { Percent } \\
\text { Recover }\end{array}$ & & $\frac{\text { Acceptable }}{\text { Range }}$ & $\underline{\mathbf{R P D}}$ \\
\hline Acenaphthene & 010913-8270S1-LCS & 50 & 33.34 & 67 & & $31-137$ & \\
\hline Acenaphthene & 010913-8270S1-LCSD & 50 & 36.58 & 73 & & $31-137$ & 9.3 \\
\hline Acenaphthene & L0109029-04-MS & 1670 & 1049 & 63 & & $47-145$ & \\
\hline Acenaphthene & L0109029-04-MSD & 1670 & 1068 & 64 & & $47-145$ & 1.8 \\
\hline Acenaphthylene & 010913-8270S1-LCS & 50 & 34.48 & 69 & & $50-120$ & \\
\hline Acenaphthylene & 010913-8270S1-LCSD & 50 & 39.46 & 79 & & $50-120$ & 13.5 \\
\hline Acenaphthylene & L0109029-04-MS & 1670 & 1090 & 65 & & $50-120$ & \\
\hline Acenaphthylene & L0109029-04-MSD & 1670 & 1168 & 70 & & $50-120$ & 6.9 \\
\hline Anthracene & 010913-8270S1-LCS & 50 & 35.38 & 71 & & $50-120$ & \\
\hline Anthracene & 010913-8270S1-LCSD & 50 & 41.43 & 83 & & $50-120$ & 15.8 \\
\hline Anthracene & L0109029-04-MS & 1670 & 1182 & 71 & & $50-120$ & \\
\hline Anthracene & L0109029-04-MSD & 1670 & 1197 & 72 & & $50-120$ & 1.3 \\
\hline Aniline & 010913-8270S1-LCS & 50 & 30.58 & 61 & & $11-77$ & \\
\hline Aniline & 010913-8270S1-LCSD & 50 & 34.43 & 69 & & $11-77$ & 11.8 \\
\hline Aniline & L0109029-04-MS & 1670 & 893 & 53 & & $11-77$ & \\
\hline Aniline & L0109029-04-MSD & 1670 & 970 & 58 & & $11-77$ & 8.3 \\
\hline Azobenzene & 010913-8270S1-LCS & 50 & 34.41 & 69 & & $22-123$ & \\
\hline Azobenzene & 010913-8270S1-LCSD & 50 & 38.82 & 78 & & $22-123$ & 12. \\
\hline Azobenzene & L0109029-04-MS & 1670 & 1098 & 66 & & $22-123$ & \\
\hline Azobenzene & L0109029-04-MSD & 1670 & 1160 & 69 & & $22-123$ & 5.5 \\
\hline Benzyl alcohol & 010913-8270S1-LCS & 50 & 32.43 & 65 & & $0-116$ & \\
\hline Benzyl alcohol & 010913-8270S1-LCSD & 50 & 32.98 & 66 & & $0-116$ & 1.7 \\
\hline Benzyl alcohol & L0109029-04-MS & 1670 & 894 & 54 & & $0-116$ & \\
\hline Benzyl alcohol & L0109029-04-MSD & 1670 & 825 & 49 & & $0-116$ & 8. \\
\hline Benzo (a) Anthracene & 010913-8270S1-LCS & 50 & 36.53 & 73 & & $50-120$ & \\
\hline Benzo (a) Anthracene & 010913-8270S1-LCSD & 50 & 37.9 & 76 & & $50-120$ & 3.7 \\
\hline Benzo (a) Anthracene & L0109029-04-MS & 1670 & 1137 & 68 & & $50-120$ & \\
\hline Benzo (a) Anthracene & L0109029-04-MSD & 1670 & 1135 & 68 & & $50-120$ & 0.2 \\
\hline Benzo (a) pyrene & 010913-8270S1-LCS & 50 & 38.66 & 77 & & $50-130$ & \\
\hline Benzo (a) pyrene & 010913-8270S1-LCSD & 50 & 43.97 & 88 & & $50-130$ & 12.9 \\
\hline Benzo (a) pyrene & L0109029-04-MS & 1670 & 1307 & 78 & & $50-130$ & \\
\hline Benzo (a) pyrene & L0109029-04-MSD & 1670 & 1285 & 77 & & $50-130$ & 1.7 \\
\hline Benzo $(g, h, i)$ perylene & 010913-8270S1-LCS & 50 & 21.79 & 44 & $\mathrm{~J}$ & $50-120$ & \\
\hline Benzo $(g, h, i)$ perylene & 010913-8270S1-LCSD & 50 & 24.13 & 48 & $\mathbf{J}$ & $50-120$ & 10.2 \\
\hline Benzo $(g, h, i)$ perylene & L0109029-04-MS & 1670 & 786 & 47 & $\mathbf{J}$ & $50-120$ & \\
\hline Benzo $(g, h, i)$ perylene & L0109029-04-MSD & 1670 & 792 & 47 & $\mathbf{J}$ & $50-120$ & 0.8 \\
\hline Benzoic Acid & 010913-8270S1-LCS & 50 & 5.86 & 12 & $\mathbf{J}$ & $50-120$ & \\
\hline Benzoic Acid & 010913-8270S1-LCSD & 50 & 6 & 12 & $\mathbf{J}$ & $50-120$ & 2.4 \\
\hline
\end{tabular}

ND - Not Detected

This report shall not be reproduced except in full, without the written approval of the laboratory. 
NEL LABORATORIES

\begin{tabular}{ll}
\hline CLIENT: & Bechtel Nevada \\
PROJECT ID: & V1249 \\
PROJECT \#: & 23081 \\
TEST: & Semi-Volatile Organic Compounds by EPA 8270C, Dec. 1996 \\
MATRIX: & Solid
\end{tabular}

- MATRIX:

PARAMETER
Benzoic Acid
Benzoic Acid
4-Bromophenyl phenyl ether
4-Bromophenyl phenyl ether
4-Bromophenyl phenyl ether
4-Bromophenyl phenyl ether
Butyl benzyl phthalate
Butyl benzyl phthalate
Carbazole
Carbazole
Carbazole
Carbazole
4-Chloroaniline
4-Chloroaniline
Chrysene
Chrysene
Chrysene
Chrysene

bis (2-Chloroethoxy) methane

bis (2-Chloroethoxy) methane

bis (2-Chloroethoxy) methane

bis (2-Chloroethoxy) methane

bis (2-Chloroethyl) ether

bis (2-Chloroethyl) ether

bis (2-Chloroethyl) ether

bis (2-Chloroethyl) ether

bis (2-chloroisopropyl) ether

bis (2-chloroisopropyl) ether

bis (2-chloroisopropyl) ether

bis (2-chloroisopropyl) ether

2-Chloronaphthalene

2-Chloronaphthalene

2-Chloronaphthalene

2-Chloronaphthalene

2-Chlorophenol

2-Chlorophenol

2-Chlorophenol

2-Chlorophenol

NEL Sample ID

L0109029-04-MS

L0109029-04-MSD

010913-8270S1-LCS

010913-8270S1-LCSD

L0109029-04-MS

L0109029-04-MSD

010913-8270S1-LCS

010913-8270S1-LCSD

010913-8270S1-LCS

010913-8270S1-LCSD

L0109029-04-MS

L0109029-04-MSD

010913-8270S1-LCS

010913-8270S1-LCSD

010913-8270S1-LCS

010913-8270S1-LCSD

L0109029-04-MS

L0109029-04-MSD

010913-8270S1-LCS

010913-8270S1-LCSD

L0109029-04-MS

L0109029-04-MSD

010913-8270S1-LCS

010913-8270S1-LCSD

L0109029-04-MS

L0109029-04-MSD

010913-8270S1-LCS

010913-8270S1-LCSD

L0109029-04-MS

L0109029-04-MSD

010913-8270S1-LCS

010913-8270S1-LCSD

L0109029-04-MS

L0109029-04-MSD

010913-8270S1-LCS

010913-8270S1-LCSD

L0109029-04-MS

L0109029-04-MSD
Spike

Spike

1670

1670

50

50

1670

1670

50

50

50

50

1670

1670

50

50

50

50

1670

1670

50

50

1670

1670

50

50

1670

1670

50

50

1670

1670

50

50

1670

1670

50

50

1670

1670
Spike

Result

594

492

35.59

44.08

1163

1270

36.54

41.29

35.65

38.66

1188

1161

23.72

31.97

36.79

38.64

1138

1091

31.96

38.19

975

1033

31.16

34.45

948

1039

33.95

37.02

1045

1130

33.83

36.98

1056

1065

35.37

35.87

1073

1139

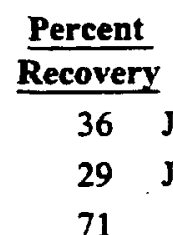

88

70

76

73

83

71

77

71

70

47

64

74

77

68

65

64

76

58

62

62

69

57

62

68

74

63

68

68

74

63

64

71

72

64

68
Acceptable

Range

$50-120$

$50-120$

$50-120$

$50-120$

21.3

$50-120$

$50-120$

8.8

$50-120$

$50-120$

$50-120$

$50-120$

$50-120$

$50-120$

1- 68

R5

$1-68$

$50-120$

$50-120$

4.9

$50-120$

$50-120$

4.2

26- 97

26- 97

17.8

26- 97

26- 97

15- 107

$15-107$

$15-107$

$15-107$

$50-120$

$50-120$

$50-120$

$50-120$

$50-120$

$50-120$

$50-120$

$50-120$

$25-102$

$25-102$

$25-102$

$25-102$

ND - Not Detected

This report shall not be reproduced except in full, without the written approval of the laboratory. 


$\begin{array}{ll}\text { CLIENT: } & \text { Bechtel Nevada } \\ \text { PROJECT ID: } & \text { V1249 } \\ \text { PROJECT \#: } & 23081 \\ \text { TEST: } & \text { Semi-Volatile Organic Compounds by EPA 8270C, Dec. } 1996 \\ \text { MATRIX: } & \text { Solid }\end{array}$

\begin{tabular}{|c|c|c|c|c|c|c|}
\hline PARAMETER & NEL Sample ID & $\begin{array}{c}\text { Spike } \\
\text { Amount }\end{array}$ & $\begin{array}{l}\text { Spike } \\
\text { Result }\end{array}$ & $\frac{\text { Percent }}{\text { Recovery }}$ & $\frac{\text { Acceptable }}{\text { Range }}$ & $\underline{\text { RPD }}$ \\
\hline 4-Chloro-3-methyl phenol & 010913-8270S1-LCS & 50 & 35.59 & 71 & $26-103$ & \\
\hline 4-Chloro-3-methyl phenol & 010913-8270S1-LCSD & 50 & 39.23 & 78 & $26-103$ & 9.7 \\
\hline 4-Chloro-3-methyl phenol & L0109029-04-MS & 1670 & 1124 & 67 & $26-103$ & \\
\hline 4-Chloro-3-methyl phenol & L0109029-04-MSD & 1670 & 1149 & 69 & $26-103$ & 2.2 \\
\hline 4-Chlorophenyl phenyl ether & 010913-8270S1-LCS & 50 & 35.26 & 71 & $50-120$ & \\
\hline 4-Chlorophenyl phenyl ether & 010913-8270S1-LCSD & 50 & 38.29 & 77 & $50-120$ & 8.2 \\
\hline 4-Chlorophenyl phenyl ether & L0109029-04-MS & 1670 & 1087 & 65 & $50-120$ & \\
\hline 4-Chlorophenyl phenyl ether & L0109029-04-MSD & 1670 & 1116 & 67 & $50-120$ & 2.6 \\
\hline Dibenz $(a, h)$ anthracene & 010913-8270S1-LCS & 50 & 26.31 & 53 & $50-120$ & \\
\hline Dibenz $(a, h)$ anthracene & 010913-8270S1-LCSD & 50 & 29.66 & 59 & $50-120$ & 12. \\
\hline Dibenzofuran & 010913-8270S1-LCS & 50 & 33.67 & 67 & $50-120$ & \\
\hline Dibenzofuran & 010913-8270S1-LCSD & 50 & 36.83 & 74 & $50-120$ & 9. \\
\hline Dibenzofuran & L0109029-04-MS & 1670 & 1056 & 63 & $50-120$ & \\
\hline Dibenzofuran & L0109029-04-MSD & 1670 & 1127 & 67 & $50-120$ & 6.5 \\
\hline 1,2-Dichlorobenzene (o-DCB) & 010913-8270S1-LCS & 50 & 30.24 & 60 & $42-93$ & \\
\hline 1,2-Dichlorobenzene (o-DCB) & 010913-8270S1-LCSD & 50 & 32.28 & 65 & $42-93$ & 6.5 \\
\hline 1,2-Dichlorobenzene (o-DCB) & L0109029-04-MS & 1670 & 906 & 54 & $42-93$ & \\
\hline 1,2-Dichlorobenzene (o-DCB) & L0109029-04-MSD & 1670 & 972 & 58 & $42-93$ & 7. \\
\hline 1,3-Dichlorobenzene (m-DCB) & 010913-8270S1-LCS & 50 & 29.58 & 59 & $41-89$ & \\
\hline 1,3-Dichlorobenzene (m-DCB) & 010913-8270S1-LCSD & 50 & 31.47 & 63 & $41-89$ & 6.2 \\
\hline 1,3-Dichlorobenzene (m-DCB) & L0109029-04-MS & 1670 & 896 & 54 & $41-89$ & \\
\hline 1,3-Dichlorobenzene (m-DCB) & L0109029-04-MSD & 1670 & 901 & 54 & $41-89$ & 0.6 \\
\hline 1,4-Dichlorobenzene (p-DCB) & 010913-8270S1-LCS & 50 & 29.7 & 59 & $42-93$ & \\
\hline 1,4-Dichlorobenzene (p-DCB) & 010913-8270S1-LCSD & 50 & 31.53 & 63 & $42-93$ & 6. \\
\hline 1,4-Dichlorobenzene (p-DCB) & L0109029-04-MS & 1670 & 871 & 52 & $42-93$ & \\
\hline 1,4-Dichlorobenzene (p-DCB) & L0109029-04-MSD & 1670 & 976 & 58 & $42-93$ & 11.4 \\
\hline 3,3'-Dichlorobenzidine & 010913-8270S1-LCS & 50 & 30.56 & 61 & $11-85$ & \\
\hline 3,3'-Dichlorobenzidine & 010913-8270S1-LCSD & 50 & 35.15 & 70 & $11-85$ & 14. \\
\hline 3,3'-Dichlorobenzidine & L0109029-04-MS & 1670 & 989 & 59 & $11-85$ & \\
\hline 3,3'-Dichlorobenzidine & L0109029-04-MSD & 1670 & 1001 & 60 & $11-85$ & 1.2 \\
\hline 2,4-Dichlorophenol & 010913-8270S1-LCS & 50 & 33.02 & 66 & $50-120$ & \\
\hline 2,4-Dichlorophenol & 010913-8270S1-LCSD & 50 & 37.95 & 76 & $50-120$ & 13.9 \\
\hline 2,4-Dichlorophenol & L0109029-04-MS & 1670 & 1080 & 65 & $50-120$ & \\
\hline 2,4-Dichlorophenol & L0109029-04-MSD & 1670 & 1153 & 69 & $50-120$ & 6.5 \\
\hline 2,4-Dimethylphenol & 010913-8270S1-LCS & 50 & 31.68 & 63 & $50-120$ & \\
\hline 2,4-Dimethylphenol & 010913-8270S1-LCSD & 50 & 35.81 & 72 & $50-120$ & 12.2 \\
\hline 2,4-Dimethylphenol & L0109029-04-MS & 1670 & 1014 & 61 & $50-120$ & \\
\hline 2,4-Dimethylphenol & L0109029-04-MSD & 1670 & 1062 & 64 & $50-120$ & 4.6 \\
\hline
\end{tabular}

ND - Not Detected

This report shall not be reproduced except in full, without the written approval of the laboratory. 
CLIENT: Bechtel Nevada

PROJECT ID: V1249

PROJECT \#: 23081

TEST: $\quad$ Semi-Volatile Organic Compounds by EPA 8270C, Dec. 1996

MATRIX: Solid

PARAMETER

4,6-Dinitro-2-methyl phenol

4,6-Dinitro-2-methyl phenol

4,6-Dinitro-2-methyl phenol

4,6-Dinitro-2-methyl phenol

2,4-Dinitrophenol

2,4-Dinitrophenol

2,4-Dinitrophenol

2,4-Dinitrophenol

2,4-Dinitrotoluene (DNT)

2,4-Dinitrotoluene (DNT)

2,4-Dinitrotoluene (DNT)

2,4-Dinitrotoluene (DNT)

2,6-Dinitrotoluene (DNT)

2,6-Dinitrotoluene (DNT)

2,6-Dinitrotoluene (DNT)

2,6-Dinitrotoluene (DNT)

Diethyl Phthalate

Diethyl Phthalate

Dimethyl phthalate

Dimethyl phthalate

Di-n-butyl phthalate

Di-n-butyl phthalate

Di-n-butyl phthalate

Di-n-butyl phthalate

Di-n-octyl phthalate

Di-n-octyl phthalate

Di-n-octyl phthalate

Di-n-octyl phthalate

sis (2-Ethylhexyl)phthalate

jis (2-Ethylhexyl)phthalate

jis (2-Ethylhexyl)phthalate

jis (2-Ethylhexyl)phthalate

Flouranthene

ilouranthene

Flourene

Flourene

Hexachlorobenzene

Hexachlorobenzene
NEL Sample ID

010913-8270S1-LCS

010913-8270S1-LCSD

L0109029-04-MS

L0109029-04-MSD

010913-8270S1-LCS

010913-8270S1-LCSD

L0109029-04-MS

L0109029-04-MSD

010913-8270S1-LCS

010913-8270S1-LCSD

L0109029-04-MS

L0109029-04-MSD

010913-8270S1-LCS

010913-8270S1-LCSD

L0109029-04-MS

L0109029-04-MSD

010913-8270S1-LCS

010913-8270S1-LCSD

010913-8270S1-LCS

010913-8270S1-LCSD

010913-8270S1-LCS

010913-8270S1-LCSD

L0109029-04-MS

L0109029-04-MSD

010913-8270S1-LCS

010913-8270S1-LCSD

L0109029-04-MS

L0109029-04-MSD

010913-8270S1-LCS

010913-8270S1-LCSD

L0109029-04-MS

L0109029-04-MSD

010913-8270S1-LCS

010913-8270S1-LCSD

010913-8270S1-LCS

010913-8270S1-LCSD

010913-8270S1-LCS

010913-8270S1-LCSD
Spike

Amount

50

50

1670

1670

50

50

1670

1670

50

50

1670

1670

50

50

1670

1670

50

50

50

50

50

50

1670

1670

50

50

1670

1670

50

50

1670

1670

50

50

50

50

50

50
Spike

Result

30.26

33.19

1150

1134

19.44

17.1

824

834

36.23

39.29

1222

1228

36.81

40.71

1241

1124

37.82

39.59

35.69

40.52

34.74

39.83

1176

1204

36.82

42.9

1263

1244

36.67

38

1140

1142

37.99

40.23

35.77

38.95

36.1

39.75

\begin{tabular}{c}
$\begin{array}{c}\text { Percent } \\
\text { Recovery }\end{array}$ \\
\hline 61 \\
66 \\
69 \\
68 \\
39 \\
34 \\
49 \\
50
\end{tabular}

72

72

79

73

74

74

81

74

67

76

79

71

81

69

80

70

72

74

86

76

74

73

76

68

68

76

80

72

78

72

80
Acceptable

Range RPD

$20-120$

$20-120$

9.2

$20-120$

$20-120$

1.4

$20-120$

$20-120$

12.8

$20-120$

$20-120$

$50-111$

$50-111$

8.1

$50-111$

$50-111$

0.5

$50-120$

$50-120$

10.1

$50-120$

$50-120$

9.9

$50-120$

$50-120$

4.6

$50-120$

$50-120$

12.7

$50-120$

$50-120$

13.7

$50-120$

$50-120$

2.4

$50-120$

$50-120$

15.3

$50-120$

$50-120$

1.5

$50-120$

$50-120$

3.6

$50-120$

$50-120$

0.2

$50-120$

$50-120$

$50-120$

$50-120$

8.5

$50-120$

$50-120$

ND - Not Detected

This report shall not be reproduced except in full, without the written approval of the laboratory. 


$\begin{array}{ll}\text { CLIENT: } & \text { Bechtel Nevada } \\ \text { PROJECT ID: } & \text { V1249 } \\ \text { PROJECT \#: } & 23081 \\ \text { TEST: } & \text { Semi-Volatile Organic Compounds by EPA 8270C, Dec. } 1996 \\ \text { MATRIX: } & \text { Solid }\end{array}$

\begin{tabular}{|c|c|c|c|c|c|c|c|}
\hline PARAMETER & NEL Sample ID & $\begin{array}{c}\text { Spike } \\
\text { Amount }\end{array}$ & $\frac{\text { Spike }}{\underline{\text { Result }}}$ & $\frac{\text { Percent }}{\text { Recovery }}$ & & $\frac{\text { Acceptable }}{\underline{\text { Range }}}$ & $\underline{\mathbf{R P D}}$ \\
\hline Hexachlorobenzene & L0109029-04-MS & 1670 & 1158 & 69 & & $50-120$ & \\
\hline Hexachlorobenzene & L0109029-04-MSD & 1670 & 1205 & 72 & & $50-120$ & 4. \\
\hline Hexachlorobutadiene & 010913-8270S1-LCS & 50 & 31.9 & 64 & & $30-120$ & \\
\hline Hexachlorobutadiene & 010913-8270S1-LCSD & 50 & 36.43 & 73 & & $30-120$ & 13.3 \\
\hline Hexachlorobutadiene & L0109029-04-MS & 1670 & 956 & 57 & & $30-120$ & \\
\hline Hexachlorobutadiene & L0109029-04-MSD & 1670 & 1040 & 62 & & $30-120$ & 8.4 \\
\hline Hexachlorocyclopentadiene & 010913-8270S1-LCS & 50 & 21.64 & 43 & & $20-120$ & \\
\hline Hexachlorocyclopentadiene & 010913-8270S1-LCSD & 50 & 22.9 & 46 & & $20-120$ & 5.7 \\
\hline Hexachlorocyclopentadiene & L0109029-04-MS & 1670 & 617 & 37 & & $20-120$ & \\
\hline Hexachlorocyclopentadiene & L0109029-04-MSD & 1670 & 590 & 35 & & $20-120$ & 4.5 \\
\hline Hexachloroethane & 010913-8270S1-LCS & 50 & 28.49 & 57 & & $30-120$ & \\
\hline Hexachloroethane & 010913-8270S1-LCSD & 50 & 30.98 & 62 & & $30-120$ & 8.4 \\
\hline Hexachloroethane & L0109029-04-MS & 1670 & 876 & 52 & & $30-120$ & \\
\hline Hexachloroethane & L0109029-04-MSD & 1670 & 904 & 54 & & $30-120$ & 3.1 \\
\hline Indeno $(1,2,3-c, d)$ pyrene & 010913-8270S1-LCS & 50 & 23.76 & 48 & $\mathbf{J}$ & $50-120$ & \\
\hline Indeno $(1,2,3-c, d)$ pyrene & 010913-8270S1-LCSD & 50 & 27.68 & 55 & & $50-120$ & 15.2 \\
\hline Indeno $(1,2,3-c, d)$ pyrene & L0109029-04-MS & 1670 & 862 & 52 & & $50-120$ & \\
\hline Indeno $(1,2,3-c, d)$ pyrene & L0109029-04-MSD & 1670 & 830 & 50 & $J$ & $50-120$ & 3.8 \\
\hline Isophorone & 010913-8270S1-LCS & 50 & 29.55 & 59 & & $24-99$ & \\
\hline Isophorone & 010913-8270S1-LCSD & 50 & 35.04 & 70 & & $24-99$ & 17. \\
\hline Isophorone & L0109029-04-MS & 1670 & 959 & 57 & & $24-99$ & \\
\hline Isophorone & L0109029-04-MSD & 1670 & 946 & 57 & & $24-99$ & 1.4 \\
\hline 2-Methylnaphthalene & 010913-8270S1-LCS & 50 & 30.99 & 62 & & $50-120$ & \\
\hline 2-Methylnaphthalene & 010913-8270S1-LCSD & 50 & 35.12 & 70 & & $50-120$ & 12.5 \\
\hline 2-Methylnaphthalene & L0109029-04-MS & 1670 & 952 & 57 & & $50-120$ & \\
\hline 2-Methylnaphthalene & L0109029-04-MSD & 1670 & 1020 & 61 & & $50-120$ & 6.9 \\
\hline 2-Methylphenol & 010913-8270S1-LCS & 50 & 31.42 & 63 & & $14-108$ & \\
\hline 2-Methylphenol & 010913-8270S1-LCSD & 50 & 34.81 & 70 & & $14-108$ & 10.2 \\
\hline 2-Methylphenol & L0109029-04-MS & 1670 & 993 & 59 & & $14-108$ & \\
\hline 2-Methylphenol & L0109029-04-MSD & 1670 & 1073 & 64 & & $14-104$ & 7.7 \\
\hline Naphthalene & 010913-8270S1-LCS & 50 & 32.31 & 65 & & $50-120$ & \\
\hline Naphthalene & 010913-8270S1-LCSD & 50 & 35.56 & 71 & & $50-120$ & 9.6 \\
\hline Naphthalene & L0109029-04-MS & 1670 & 991 & 59 & & $50-120$ & \\
\hline Naphthalene & L0109029-04-MSD & 1670 & 1069 & 64 & & $50-120$ & 7.6 \\
\hline 2-Nitroaniline & 010913-8270S1-LCS & 50 & 33.52 & 67 & & $30-120$ & \\
\hline 2-Nitroaniline & 010913-8270S1-LCSD & 50 & 40.62 & 81 & & $30-120$ & 19.2 \\
\hline 2-Nitroaniline & L0109029-04-MS & 1670 & 1108 & 66 & & $30-120$ & \\
\hline 2-Nitroaniline & L0109029-04-MSD & 1670 & 1223 & 73 & & $30-120$ & 9.9 \\
\hline
\end{tabular}

ND - Not Detected

This report shall not be reproduced except in full, without the written approval of the laboratory. 


$\begin{array}{ll}\text { CLIENT: } & \text { Bechtel Nevada } \\ \text { PROJECT DD: } & \text { V1249 } \\ \text { PROJECT \#: } & 23081 \\ \text { TEST: } & \text { Semi-Volatile Organic Compounds by EPA 8270C, Dec. } 1996 \\ \text { MATRIX: } & \text { Solid }\end{array}$

\begin{tabular}{|c|c|c|c|c|c|c|}
\hline PARAMETER & NEL Sample ID & $\frac{\text { Spike }}{\text { Amount }}$ & $\begin{array}{l}\text { Spike } \\
\text { Result }\end{array}$ & $\frac{\text { Percent }}{\text { Recovery }}$ & $\frac{\text { Acceptable }}{\text { Range }}$ & $\underline{\text { RPD }}$ \\
\hline 3-Nitroaniline & 010913-8270S1-LCS & 50 & 34.2 & 68 & $30-120$ & \\
\hline 3-Nitroaniline & 010913-8270S1-LCSD & 50 & 35.53 & 71 & $30-120$ & 3.8 \\
\hline 3-Nitroaniline & L0109029-04-MS & 1670 & 1018 & 61 & $30-120$ & \\
\hline 3-Nitroaniline & L0109029-04-MSD & 1670 & 1030 & 62 & $30-120$ & 1.2 \\
\hline 4-Nitroaniline & 010913-8270S1-LCS & 50 & 29.29 & 59 & $30-120$ & \\
\hline 4-Nitroaniline & 010913-8270S1-LCSD & 50 & 35.5 & 71 & $30-120$ & 19.2 \\
\hline 4-Nitroaniline & L0109029-04-MS & 1670 & 1022 & 61 & $30-120$ & \\
\hline 4-Nitroaniline & L0109029-04-MSD & 1670 & 1064 & 64 & $30-120$ & 4. \\
\hline 2-Nitrophenol & 010913-8270S1-LCS & 50 & 34.9 & 70 & $42-86$ & \\
\hline 2-Nitrophenol & 010913-8270S1-LCSD & 50 & 35.34 & 71 & $42-86$ & 1.3 \\
\hline 2-Nitrophenol & L0109029-04-MS & 1670 & 990 & 59 & $42-86$ & \\
\hline 2-Nitrophenol & L0109029-04-MSD & 1670 & 1017 & 61 & $42-86$ & 2.7 \\
\hline 4-Nitrophenol & 010913-8270S1-LCS & 50 & 29.29 & 59 & $22-151$ & \\
\hline 4-Nitrophenol & 010913-8270S1-LCSD & 50 & 35.5 & 71 & $22-151$ & 19.2 \\
\hline 4-Nitrophenol & L0109029-04-MS & 1670 & 1022 & 61 & $22-151$ & \\
\hline 4-Nitrophenol & L0109029-04-MSD & 1670 & 1064 & 64 & $22-151$ & 4. \\
\hline Nitrobenzene & 010913-8270S1-LCS & 50 & 31.49 & 63 & $23-103$ & \\
\hline Nitrobenzene & 010913-8270S1-LCSD & 50 & 35.94 & 72 & $23-103$ & 13.2 \\
\hline Nitrobenzene & L0109029-04-MS & 1670 & 998 & 60 & $23-103$ & \\
\hline Nitrobenzene & L0109029-04-MSD & 1670 & 1066 & 64 & $23-103$ & 6.6 \\
\hline N-Nitrosodimethylamine & 010913-8270S1-LCS & 50 & 34.9 & 70 & $9-97$ & \\
\hline N-Nitrosodimethylamine & 010913-8270S1-LCSD & 50 & 37.38 & 75 & $9-97$ & 6.9 \\
\hline N-Nitrosodimethylamine & L0109029-04-MS & 1670 & 1036 & 62 & $9-97$ & \\
\hline N-Nitrosodimethylamine & L0109029-04-MSD & 1670 & 1014 & 61 & $9-97$ & 2.1 \\
\hline N-Nitrosodiphenylamine & 010913-8270S1-LCS & 50 & 35.99 & 72 & $50-120$ & \\
\hline N-Nitrosodiphenylamine & 010913-8270S1-LCSD & 50 & 41.03 & 82 & $50-120$ & 13.1 \\
\hline N-Nitrosodiphenylamine & L0109029-04-MS & 1670 & 1153 & 69 & $50-120$ & \\
\hline N-Nitrosodiphenylamine & L0109029-04-MSD & 1670 & 1228 & 74 & $50-120$ & 6.3 \\
\hline N-Nitrosodi-n-propylamine & 010913-8270S1-LCS & 50 & 34.07 & 68 & $41-126$ & \\
\hline N-Nitrosodi-n-propylamine & 010913-8270S1-LCSD & 50 & 34.83 & 70 & $41-126$ & 2.2 \\
\hline N-Nitrosodi-n-propylamine & L0109029-04-MS & 1670 & 994 & 60 & $41-126$ & \\
\hline N-Nitrosodi-n-propylamine & L0109029-04-MSD & 1670 & 1062 & 64 & $41-126$ & 6.6 \\
\hline Pentachlorophenol & 010913-8270S1-LCS & 50 & 26.49 & 53 & $17-109$ & \\
\hline Pentachlorophenol & 010913-8270S1-LCSD & 50 & 29.89 & 60 & $17-109$ & 12.1 \\
\hline Pentachlorophenol & L0109029-04-MS & 1670 & 1032 & 62 & $17-109$ & \\
\hline Pentachlorophenol & L0109029-04-MSD & 1670 & 1110 & 66 & $17-109$ & 7.3 \\
\hline Phenanthrene & 010913-8270S1-LCS & 50 & 33.71 & 67 & $50-120$ & \\
\hline Phenanthrene & 010913-8270S1-LCSD & 50 & 38.7 & 77 & $50-120$ & 13.8 \\
\hline
\end{tabular}

ND - Not Detected

This report shall not be reproduced except in full, without the written approval of the laboratory. 


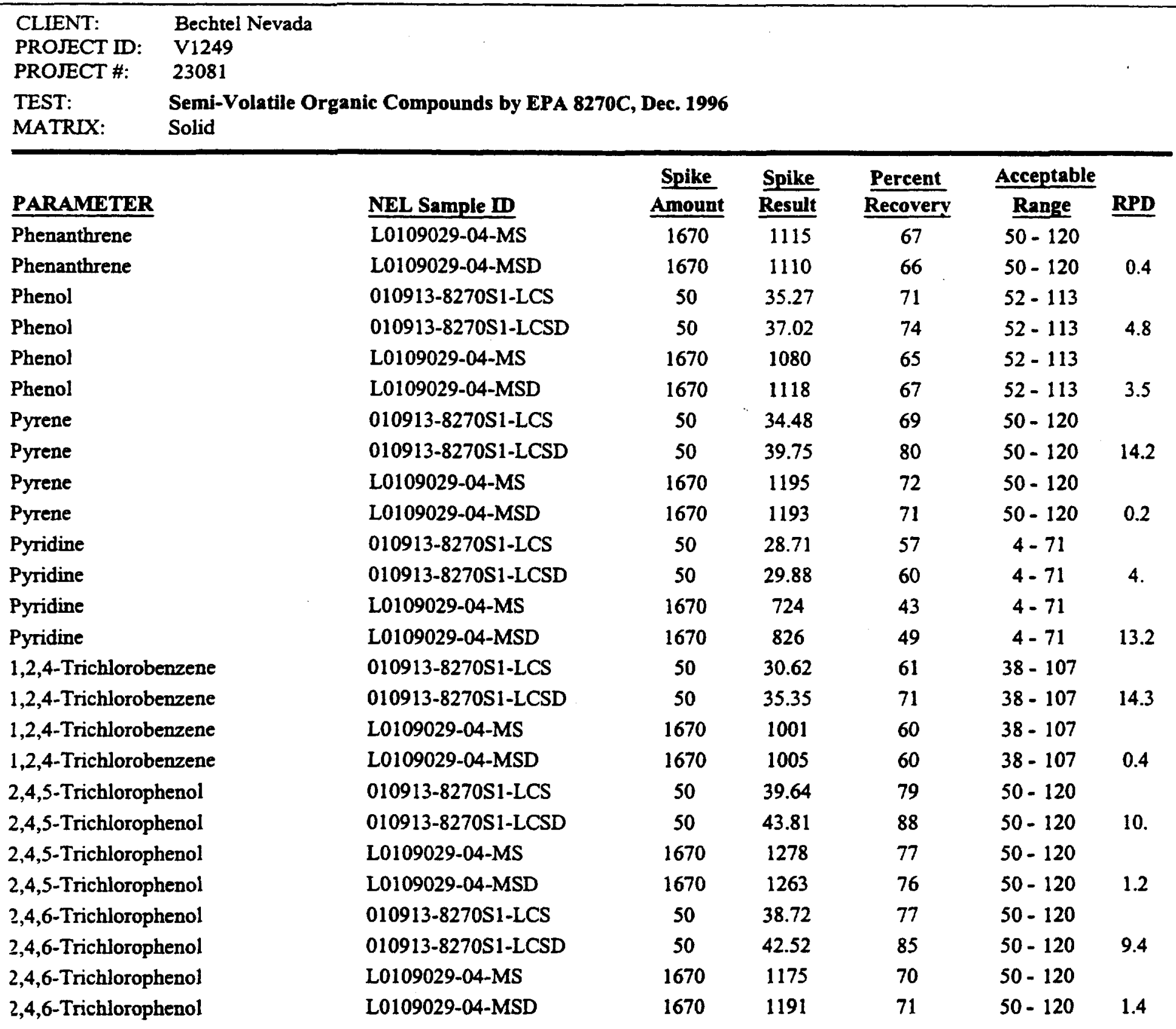

ND - Not Detected

This report shall not be reproduced except in full, without the written approval of the laboratory. 


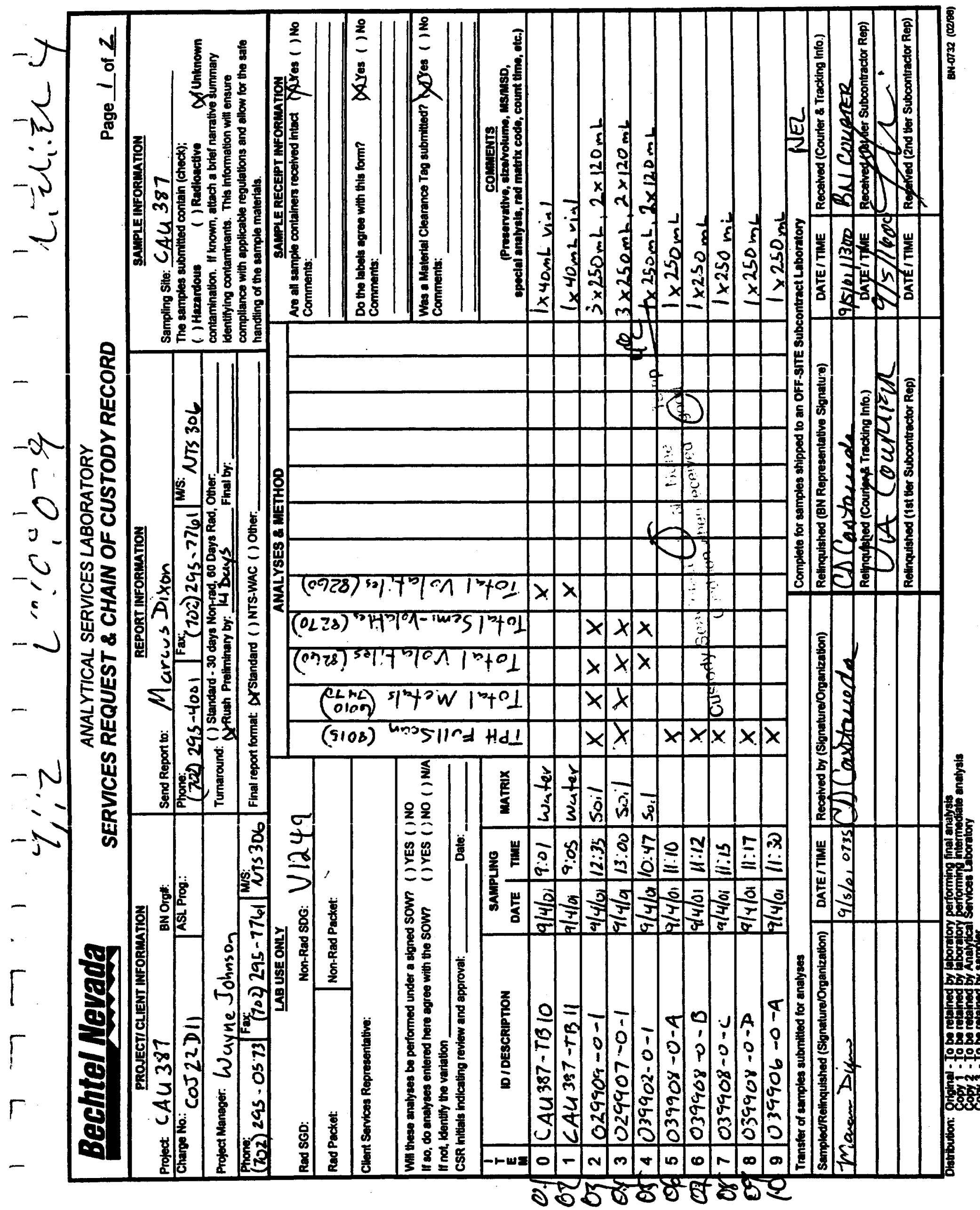




$\begin{array}{ll}\text { CLIENT: } & \text { Bechtel Nevada } \\ & \text { P.O. Box 98521, M/S NTS273 } \\ & \text { Las Vegas, NV 89193-8521 } \\ \text { ATTN: } & \text { Ted Redding }\end{array}$

PROJECT NAME: $\quad$ V1271 PROJECT NUMBER: 23081

Attached are the analytical results for samples in support of the above referenced project.

Samples submitted for this project were not sampled by NEL Laboratories. Samples were received by NEL in good condition, under chain of custody on 9/19/01.

Should you have any questions or comments, please feel free to contact our Client Services department at (702) 657-1010.

\section{Some QA results have been flagged as follows:}

J - This concentration should be considered an estimate due laboratory control sample failure.

Jl - The batch MS and/or MSD were outside acceptance limits. The batch LCS was acceptable.
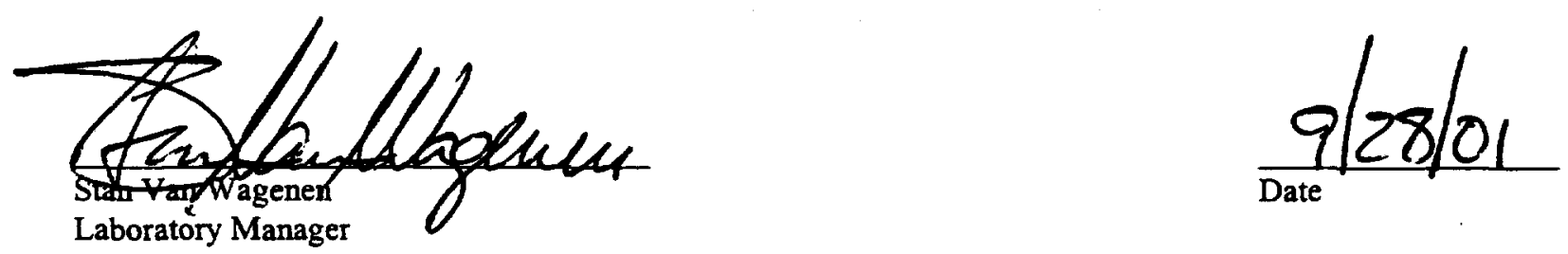

\section{CERTIFICATIONS:}

\begin{tabular}{llll}
\hline & Reno & Las Vegas & S. California \\
\cline { 2 - 4 } Arizona & AZ0520 & AZ0518 & AZ0605 \\
California & 1707 & 2002 & 2264 \\
US Army Corps & Certified & Certified & \\
of Engineers & & &
\end{tabular}

\begin{tabular}{llll} 
& Reno & Las Vegas & S. California \\
\cline { 2 - 4 } Idaho & Certified & Certified & \\
Montana & Certified & Certified & \\
Nevada & NV033 & NV052 & CA084 \\
L.A.C.S.D. & & & 10228
\end{tabular}




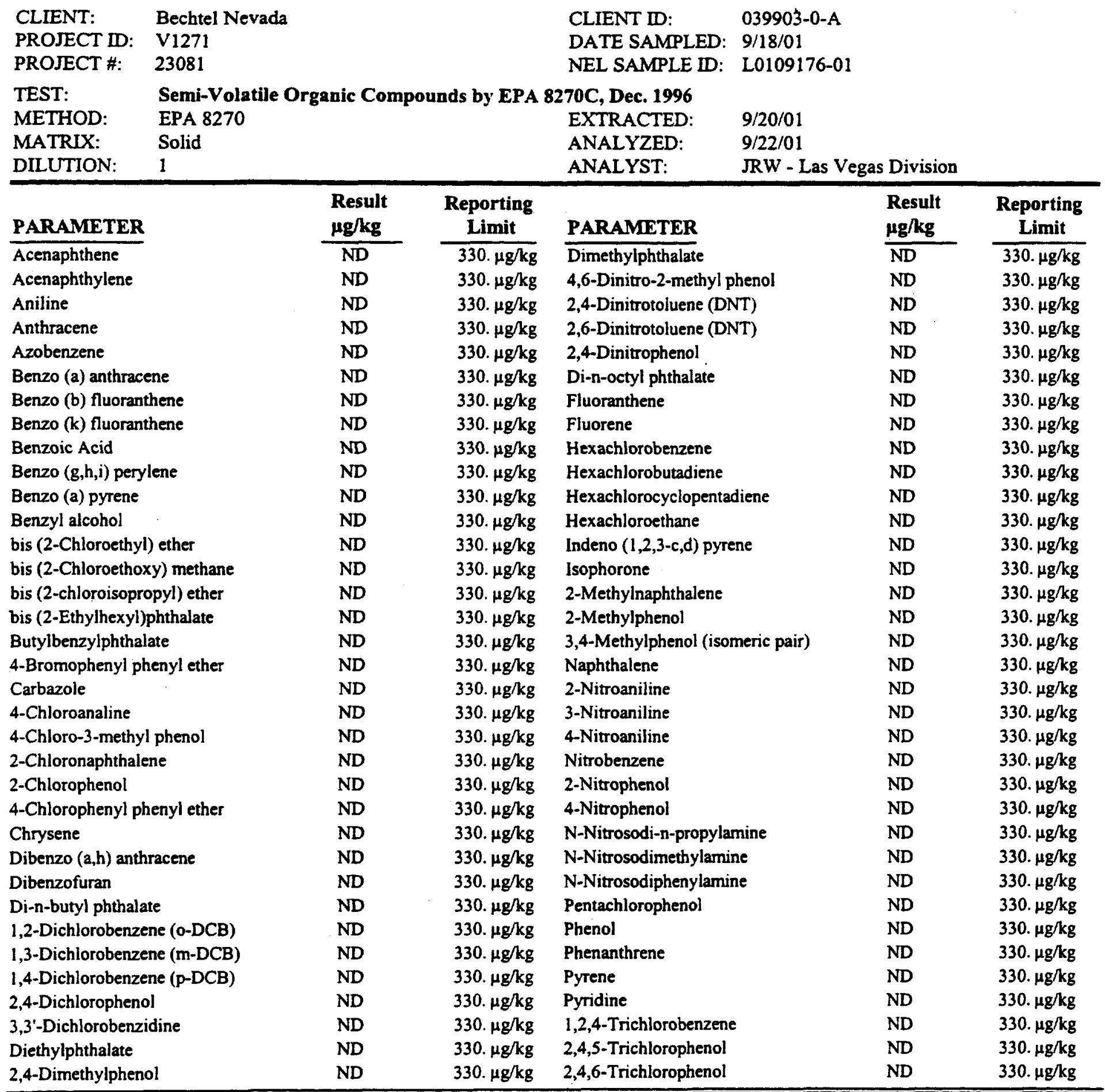

QUALITY CONTROL DATA:

\section{Surrogate}

2,4,6-Tribromophenol

2-Fluorobiphenyl

2-Fluorophenol

Nitrobenzene-d5

p-Terphenyl-d14

Phenol-d6

\begin{tabular}{c} 
\% Recovery \\
\hline 48 \\
44 \\
36 \\
42 \\
48 \\
41
\end{tabular}

Acceptable Range

$19-122$

$30-115$

$25-121$

$23-120$

$18-137$

$24-113$

ND - Not Detected

This report shall not be reproduced except in full, without the written approval of the laboratory. 
CLIENT: Bechtel Nevada

PROJECT ID: V1271

PROJECT \#: 23081
CLIENT ID: $\quad$ 039901-0-A

DATE SAMPLED: $9 / 18 / 01$

NEL SAMPLE ID: L0109176-02

- TEST:

METHOD

MATRIX:

Semi-Volatile Organic Compounds by EPA 8270 C, Dec. 1996

Solid

- DILUTION: 1

Result Reporting

PARAMETER

Acenaphthene

Acenaphthylene

Aniline

Anthracene

Azobenzene

Benzo (a) anthracene

Benzo (b) fluoranthene

Benzo (k) fluoranthene

Benzoic Acid

Benzo (g,h,i) perylene

Benzo (a) pyrene

Benzyl alcohol

bis (2-Chloroethyl) ether

bis (2-Chloroethoxy) methane

bis (2-chloroisopropyl) ether

bis (2-Ethylhexyi)phthalate

Butylbenzylphthalate

4-Bromophenyl phenyl ether

Carbazole

4-Chloroanaline

4-Chloro-3-methyl phenol

2-Chloronaphthalene

2-Chlorophenol

4-Chlorophenyl phenyl ether

Chrysene

Dibenzo $(\mathrm{a}, \mathrm{h})$ anthracene

Dibenzofuran

Di-n-butyl phthalate

1,2-Dichlorobenzene (o-DCB)

1,3-Dichlorobenzene (m-DCB)

1,4-Dichlorobenzene ( $p$-DCB)

2,4-Dichlorophenol

3,3'-Dichlorobenzidine

Diethyiphthalate

2,4-Dimethylphenol

\begin{tabular}{c}
$\mu \mathrm{g} / \mathrm{kg}$ \\
\hline $\mathrm{ND}$ \\
$\mathrm{ND}$ \\
$\mathrm{ND}$ \\
$\mathrm{ND}$ \\
$\mathrm{ND}$ \\
$\mathrm{ND}$ \\
$\mathrm{ND}$
\end{tabular}

340

ND

ND

ND

ND

ND

ND

ND

ND

ND

ND

ND

ND

ND

ND

ND

ND

ND

ND

ND

ND

ND

ND

ND

ND

ND

ND

ND
EXTRACTED:

ANALYZED:

ANALYST:
9/20/01

9/22/01

JRW - Las Vegas Division

QUALITY CONTROL DATA:

Surrogate

2,4,6-Tribromophenol

2-Fluorobiphenyl

2-Fluorophenol

Nitrobenzene-d5

p-Terphenyl-d14

Phenol-d6
Reporting

\begin{tabular}{l} 
Limit \\
\hline $330 . \mu \mathrm{g} / \mathrm{kg}$ \\
330. $\mu \mathrm{g} / \mathrm{kg}$
\end{tabular}

330. $\mu \mathrm{g} / \mathrm{kg}$

330. $\mu \mathrm{g} / \mathrm{kg}$

330. $\mu \mathrm{g} / \mathrm{kg}$

330. $\mu g / \mathrm{kg}$

330. $\mu \mathrm{g} / \mathrm{kg}$

330. $\mu \mathrm{g} / \mathrm{kg}$

$330 . \mu \mathrm{g} / \mathrm{kg}$

330. $\mu \mathrm{g} / \mathrm{kg}$

$330 . \mu \mathrm{g} / \mathrm{kg}$

$330 . \mu \mathrm{g} / \mathrm{kg}$

330. $\mu \mathrm{g} / \mathrm{kg}$

330. $\mu \mathrm{g} / \mathrm{kg}$

330. $\mu \mathrm{g} / \mathrm{kg}$

330. $\mu \mathrm{g} / \mathrm{kg}$

330. $\mu \mathrm{g} / \mathrm{kg}$

330. $\mu \mathrm{g} / \mathrm{kg}$

$330 . \mu \mathrm{g} / \mathrm{kg}$

330. $\mu \mathrm{g} / \mathrm{kg}$

330. $\mu \mathrm{g} / \mathrm{kg}$

330. $\mu \mathrm{g} / \mathrm{kg}$

330. $\mu \mathrm{g} / \mathrm{kg}$

330. $\mu \mathrm{g} / \mathrm{kg}$

$330 . \mu \mathrm{g} / \mathrm{kg}$

$330 . \mu \mathrm{g} / \mathrm{kg}$

330. $\mu \mathrm{g} / \mathrm{kg}$

$330 . \mu \mathrm{g} / \mathrm{kg}$

330. $\mu \mathrm{g} / \mathrm{kg}$

330. $\mu \mathrm{g} / \mathrm{kg}$

330. $\mu \mathrm{g} / \mathrm{kg}$

$330 . \mu \mathrm{g} / \mathrm{kg}$

330. $\mu \mathrm{g} / \mathrm{kg}$

330. $\mu \mathrm{g} / \mathrm{kg}$

330. $\mu \mathrm{g} / \mathrm{kg}$
PARAMETER

Dimethylphthalate

4,6-Dinitro-2-methyl phenol

2,4-Dinitrotoluene (DNT)

2,6-Dinitrotoluene (DNT)

2,4-Dinitrophenol

Di-n-octyl phthalate

Fluoranthene

Fluorene

Hexachlorobenzene

Hexachlorobutadiene

Hexachlorocyclopentadiene

Hexachloroethane

Indeno (1,2,3-c,d) pyrene

Isophorone

2-Methylnaphthalene

2-Methylphenol

3,4-Methylphenol (isomeric pair)

Naphthalene

2-Nitroaniline

3-Nitroaniline

4-Nitroaniline

Nitrobenzene

2-Nitrophenol

4-Nitrophenol

N-Nitrosodi-n-propylamine

N-Nitrosodimethylamine

N-Nitrosodiphenylamine

Pentachlorophenol

Phenol

Phenanthrene

Pyrene

Pyridine

1,2,4-Trichlorobenzene

2,4,5-Trichlorophenol

2,4,6-Trichlorophenol

Result

Reporting

$\mu \mathrm{g} / \mathrm{kg}$

ND

ND

ND

ND

ND

ND

510

ND

ND

ND

ND

ND

ND

ND

ND

ND

ND

ND

ND

ND

ND

ND

ND

ND

ND

ND

ND

ND

ND

500

630

ND

ND

ND

ND
Limit

330. $\mu \mathrm{g} / \mathrm{kg}$

330. $\mu \mathrm{g} / \mathrm{kg}$

330. $\mu \mathrm{g} / \mathrm{kg}$

330. $\mu \mathrm{g} / \mathrm{kg}$

330. $\mu \mathrm{g} / \mathrm{kg}$

330. $\mu \mathrm{g} / \mathrm{kg}$

$330 . \mu \mathrm{g} / \mathrm{kg}$

$330 . \mu \mathrm{g} / \mathrm{kg}$

$330 . \mu \mathrm{g} / \mathrm{kg}$

$330 . \mu \mathrm{g} / \mathrm{kg}$

$330 . \mu \mathrm{g} / \mathrm{kg}$

$330 . \mu \mathrm{g} / \mathrm{kg}$

330. $\mu \mathrm{g} / \mathrm{kg}$

$330 . \mu \mathrm{g} / \mathrm{kg}$

330. $\mu \mathrm{g} / \mathrm{kg}$

330. $\mu \mathrm{g} / \mathrm{kg}$

330. $\mu \mathrm{g} / \mathrm{kg}$

330. $\mu g / \mathrm{kg}$

330. $\mu \mathrm{g} / \mathrm{kg}$

330. $\mu \mathrm{g} / \mathrm{kg}$

330. $\mu \mathrm{g} / \mathrm{kg}$

330. $\mu \mathrm{g} / \mathrm{kg}$

330. $\mu \mathrm{g} / \mathrm{kg}$

$330 . \mu \mathrm{g} / \mathrm{kg}$

$330 . \mu \mathrm{g} / \mathrm{kg}$

$330 . \mu \mathrm{g} / \mathrm{kg}$

$330 . \mu \mathrm{g} / \mathrm{kg}$

$330 . \mu \mathrm{g} / \mathrm{kg}$

330. $\mu \mathrm{g} / \mathrm{kg}$

330. $\mu \mathrm{g} / \mathrm{kg}$

330. $\mu \mathrm{g} / \mathrm{kg}$

330. $\mu \mathrm{g} / \mathrm{kg}$

330. $\mu \mathrm{g} / \mathrm{kg}$

330. $\mu \mathrm{g} / \mathrm{kg}$

ND - Not Detected

This report shall not be reproduced except in full, without the written approval of the laboratory.

Acceptable Range

70

50

42

42

62

49
$19-122$

$30-115$

$25-121$

$23-120$

$18-137$

$24-113$ 


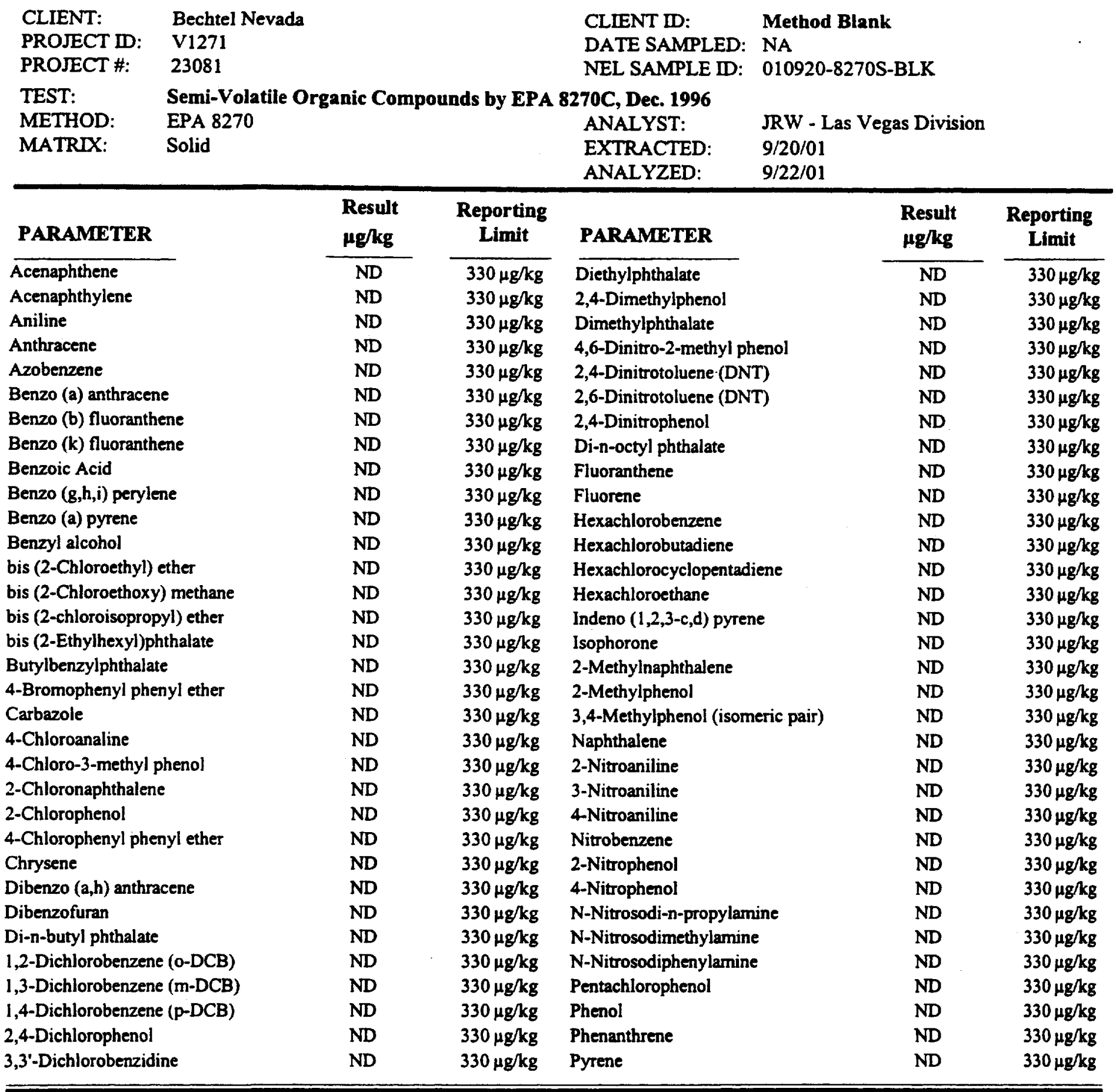

QUALITY CONTROL DATA:

\section{Surrogate}

2,4,6-Tribromophenol

2-Fluorobiphenyl

2-Fluorophenol

Nitrobenzene-d5

p-Terphenyl-d14

Phenol-d6.

\begin{tabular}{c} 
\% Recovery \\
\hline 56 \\
57 \\
53 \\
57 \\
61 \\
55
\end{tabular}

Acceptable Range

$19-122$

$30-115$

$25-121$

$23-120$

$18-137$

$24-113$

ND - Not Detected

This report shall not be reproduced except in full, without the written approval of the laboratory. 


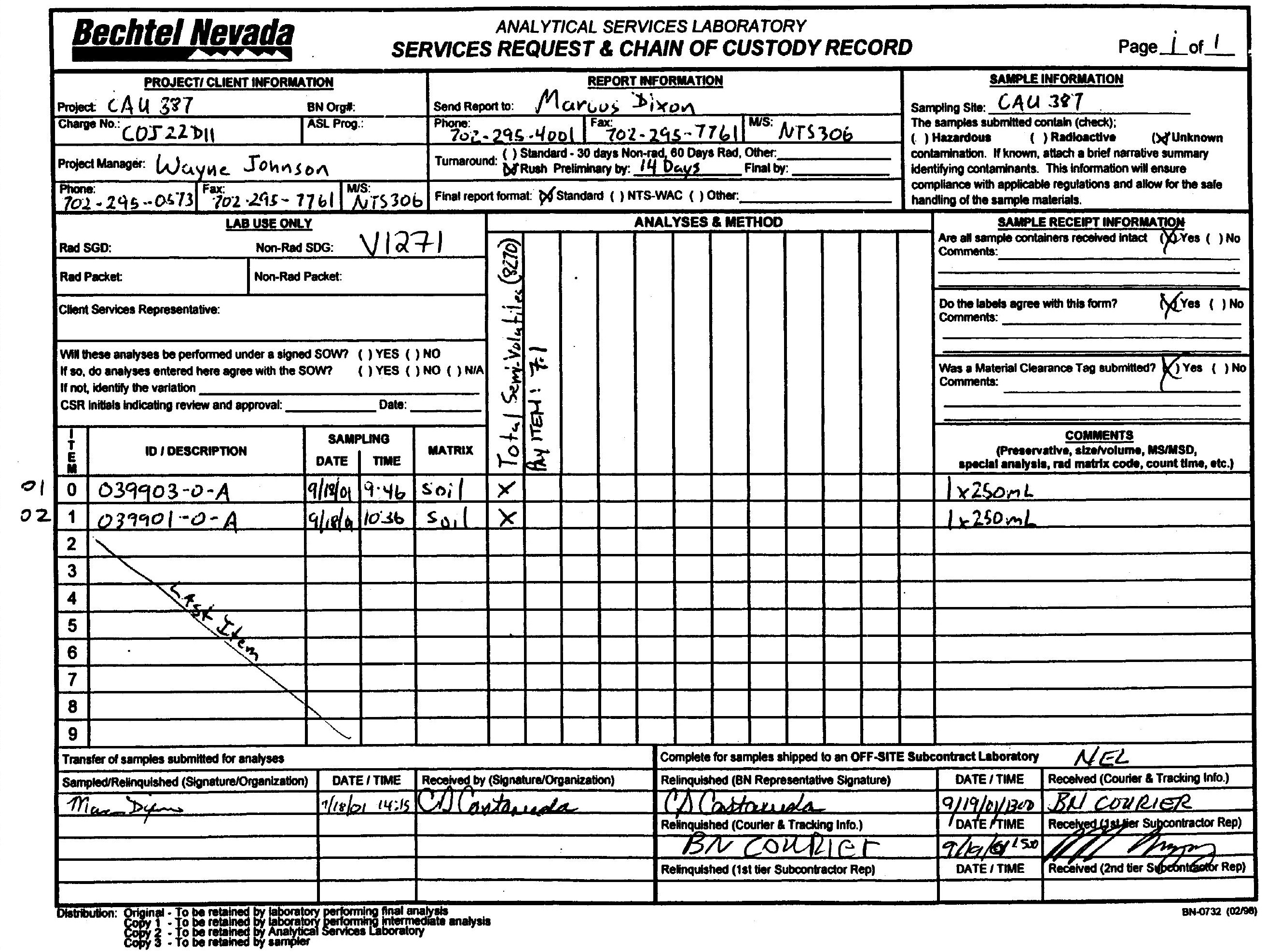


CLIENT: Bechtel Nevada

P.O. Box 98521 , M/S NTS273

Las Vegas, NV 89193-8521

ATTN: $\quad$ Ted Redding

PROJECT NAME: V1221

NEL ORDER ID: L0107296

PROJECT NUMBER: 23081

Attached are the analytical results for samples in support of the above referenced project.

Samples submitted for this project were not sampled by NEL Laboratories. Samples were received by NEL in good condition, under chain of custody on 7/30/01.

Should you have any questions or comments, please feel free to contact our Client Services department at (702) 657-1010.

Some results have been flagged as follows:

J - This concentration should be considered an estimate due laboratory control sample failure.

Some QA results have been flagged as follows:

J - This concentration should be considered an estimate due laboratory control sample failure.

$\pi$ - The batch MS and/or MSD were outside acceptance limits. The batch LCS was acceptable.
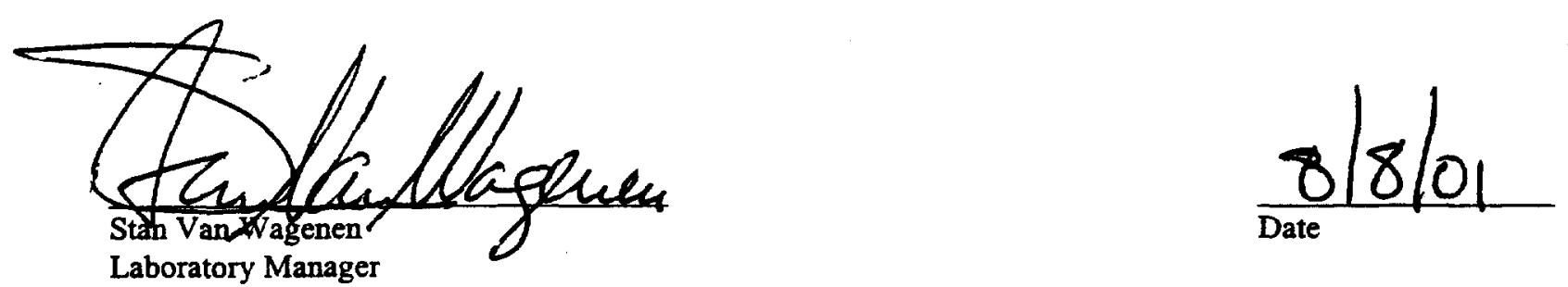

\section{CERTIFICATIONS:}

Arizona

California

US Army Corps Certified Certified

of Engineers

\begin{tabular}{lrc} 
Reno & Las Vegas & S. Califormia \\
\hline AZ0520 & AZ0518 & AZ0605
\end{tabular}

$1707 \quad 2002 \quad 2264$




\begin{tabular}{|c|c|c|c|c|c|c|c|c|c|c|}
\hline $\begin{array}{l}\text { CLIENT: } \\
\text { PROJECT ID: } \\
\text { PROJECT \#: }\end{array}$ & $\begin{array}{l}\text { Bechtel Nevada } \\
\text { V1221 } \\
23081\end{array}$ & & & & $\begin{array}{l}\text { CLIENT ID: } \\
\text { DATE SAMPLED: } \\
\text { NEL SAMPLE ID: }\end{array}$ & $\begin{array}{l}039901-0-B \\
7 / 25 / 01 \\
\text { L0107296-03 }\end{array}$ & & & & च \\
\hline $\begin{array}{l}\text { TEST: } \\
\text { METHOD: } \\
\text { MATRIX: } \\
\text { DILUTION: }\end{array}$ & $\begin{array}{l}\text { Semi-Volatile C } \\
\text { EPA } 8270 \\
\text { Solid } \\
1 \\
\end{array}$ & anic Co & $\mathrm{mp}$ & Inds by EPA & $\begin{array}{l}\text { 270C, Dec. } 1996 \\
\text { EXTRACTED: } \\
\text { ANALYZED: } \\
\text { ANALYST: }\end{array}$ & $\begin{array}{l}7 / 31 / 01 \\
7 / 31 / 01 \\
\text { JRW - Las Veg: }\end{array}$ & s Divis & & & \\
\hline PARAMETER & & $\begin{array}{l}\text { Result } \\
\mu \mathrm{g} / \mathrm{kg}\end{array}$ & & $\begin{array}{c}\text { Reporting } \\
\text { Limit } \\
\end{array}$ & PARAMETER & & $\begin{array}{l}\text { Resul } \\
\mu \mathrm{g} / \mathrm{kg}\end{array}$ & & $\begin{array}{c}\text { Reporting } \\
\text { Limit }\end{array}$ & \\
\hline Acenaphthene & & ND & & $\overline{330 . \mu \mathrm{g} / \mathrm{kg}}$ & $\overline{\text { Dimethylphthalate }}$ & & ND & & $330 . \mu \mathrm{g} / \mathrm{kg}$ & \\
\hline Acenaphthylene & & ND & & $330 . \mu \mathrm{g} / \mathrm{kg}$ & 4,6-Dinitro-2-methyl & henol & $\mathrm{ND}$ & & $330 . \mu \mathrm{g} / \mathrm{kg}$ & \\
\hline Aniline & & ND & & $330 . \mu \mathrm{g} / \mathrm{kg}$ & 2,4-Dinitrotoluene (D) & & ND & & $330 . \mu \mathrm{g} / \mathrm{kg}$ & \\
\hline Anthracene & & ND & & $330 . \mu \mathrm{g} / \mathrm{kg}$ & 2,6-Dinitrotoluene (Dl & & ND & & $330 . \mu \mathrm{g} / \mathrm{kg}$ & \\
\hline Azobenzene & & ND & & $330 . \mu \mathrm{g} / \mathrm{kg}$ & 2,4-Dinitrophenol & & ND & & $330 . \mu \mathrm{g} / \mathrm{kg}$ & \\
\hline Benzo (a) anthrac & & ND & & $330 . \mu \mathrm{g} / \mathrm{kg}$ & Di-n-octyl phthalate & & ND & & $330 . \mu \mathrm{g} / \mathrm{kg}$ & \\
\hline Benzo (b) fluoran & thene & ND & & $330 . \mu \mathrm{g} / \mathrm{kg}$ & Fluoranthene & & ND & & $330 . \mu \mathrm{g} / \mathrm{kg}$ & \\
\hline Benzo (k) fluoran & thene & ND & & $330 . \mu \mathrm{g} / \mathrm{kg}$ & Fluorene & & ND & & 330. $\mu g / \mathbf{k g}$ & \\
\hline Benzoic Acid & & ND & $\mathrm{J}$ & $330 . \mu \mathrm{g} / \mathrm{kg}$ & Hexachlorobenzene & & ND & & $330 . \mu \mathrm{g} / \mathrm{kg}$ & \\
\hline Benzo $(g, h, i)$ pery & lene & ND & & $330 . \mu \mathrm{g} / \mathrm{kg}$ & Hexachlorobutadiene & & $\mathrm{ND}$ & & $330 . \mu g / k g$ & \\
\hline Benzo (a) pyrene & & ND & & 330. $\mu \mathrm{g} / \mathrm{kg}$ & Hexachlorocyclopenta & diene & ND & & 330. $\mu \mathrm{g} / \mathrm{kg}$ & \\
\hline Benzyl alcohol & & ND & & 330. $\mu \mathrm{g} / \mathrm{kg}$ & Hexachloroethane & & ND & & 330. $\mu \mathrm{g} / \mathrm{kg}$ & \\
\hline bis (2-Chloroethyl & ) ether & ND & & $330 . \mu \mathrm{g} / \mathrm{kg}$ & Indeno $(1,2,3-c, d)$ pyre & & ND & & $330 . \mu \mathrm{g} / \mathrm{kg}$ & \\
\hline bis (2-Chloroethos & xy) methane & ND & & $330 . \mu \mathrm{g} / \mathrm{kg}$ & Isophorone & & $\mathrm{ND}$ & & $330 . \mu \mathrm{g} / \mathrm{kg}$ & \\
\hline bis (2-chloroisopr & opyl) ether & ND & & $330 . \mu \mathrm{g} / \mathrm{kg}$ & 2-Methylnaphthalene & & ND & & 330. $\mu \mathrm{g} / \mathrm{kg}$ & \\
\hline bis (2-Ethylhexyl) & phthalate & ND & & $330 . \mu \mathrm{g} / \mathrm{kg}$ & 2-Methylphenol & & ND & & $330 . \mu \mathrm{g} / \mathrm{kg}$ & \\
\hline Butylbenzylphthal & & ND & & $330 . \mu \mathrm{g} / \mathrm{kg}$ & 3,4-Methylphenol (iso & neric pair) & ND & & 330. $\mu \mathrm{g} / \mathrm{kg}$ & \\
\hline 4-Bromophenyl pl & nenyl ether & ND & J & $330 . \mu \mathrm{g} / \mathrm{kg}$ & Naphthalene & & ND & & 330. $\mu \mathrm{g} / \mathrm{kg}$ & \\
\hline Carbazole & & ND & & $330 . \mu \mathrm{g} / \mathrm{kg}$ & 2-Nitroaniline & & ND & & 330. $\mu \mathrm{g} / \mathrm{kg}$ & \\
\hline 4-Chloroanaline & & ND & & $330 . \mu \mathrm{g} / \mathrm{kg}$ & 3-Nitroaniline & & ND & & $330 . \mu \mathrm{g} / \mathrm{kg}$ & \\
\hline 4-Chioro-3-methy & I phenol & ND & & $330 . \mu \mathrm{g} / \mathrm{kg}$ & 4-Nitroaniline & & ND & & $330 . \mu g / k g$ & \\
\hline 2-Chloronaphthale & & ND & & 330. $\mu \mathrm{g} / \mathrm{kg}$ & Nitrobenzene & & ND & & 330. $\mu \mathrm{g} / \mathrm{kg}$ & \\
\hline 2-Chlorophenol & & ND & & 330. $\mu \mathrm{g} / \mathrm{kg}$ & 2-Nitrophenol & & ND & & 330. $\mathrm{\mu g} / \mathrm{kg}$ & \\
\hline 4-Chlorophenyl pl & henyl ether & ND & & $330 . \mu \mathrm{g} / \mathrm{kg}$ & 4-Nitrophenol & & ND & & $330 . \mu \mathrm{g} / \mathrm{kg}$ & \\
\hline Chrysene & & ND & & 330. $\mu \mathrm{g} / \mathrm{kg}$ & N-Nitrosodi-n-propyla & nine & ND & & 330. $\mu \mathrm{g} / \mathrm{kg}$ & \\
\hline Dibenzo $(\mathrm{a}, \mathrm{h})$ anth & iracene & ND & & $330 . \mu \mathrm{g} / \mathrm{kg}$ & N-Nitrosodimethylami & & ND & & 330. $\mu \mathrm{g} / \mathrm{kg}$ & \\
\hline Dibenzofuran & & ND & & $330 . \mu \mathrm{g} / \mathrm{kg}$ & N-Nitrosodiphenylami & & ND & & 330. $\mu \mathrm{g} / \mathrm{kg}$ & \\
\hline Di-n-butyl phthala & & ND & & $330 . \mu \mathrm{g} / \mathrm{kg}$ & Pentachlorophenol & & ND & & 330. $\mu \mathrm{g} / \mathrm{kg}$ & \\
\hline 1,2-Dichlorobenze & ene $(0-D C B)$ & ND & & $330 . \mu \mathrm{g} / \mathrm{kg}$ & Phenol & & ND & & 330. $\mu \mathrm{g} / \mathrm{kg}$ & \\
\hline 1,3-Dichlorobenze & ene ( $m$-DCB) & ND & & $330 . \mu g / k g$ & Phenanthrene & & ND & & $330 . \mu g / k g$ & \\
\hline 1,4-Dichlorobenze & ene ( $p-D C B)$ & ND & & 330. $\mu \mathrm{g} / \mathrm{kg}$ & Pyrene & & ND & $\mathbf{j}$ & 330. $\mu \mathrm{g} / \mathrm{kg}$ & \\
\hline 2,4-Dichlorophen & & ND & J & 330. $\mu \mathrm{g} / \mathrm{kg}$ & Pyridine & & ND & & 330. $\mu \mathrm{g} / \mathrm{kg}$ & \\
\hline 3,3'-Dichlorobenz & idine & ND & & $330 . \mu \mathrm{g} / \mathrm{kg}$ & 1,2,4-Trichlorobenzen & & ND & & 330. $\mathrm{\mu g} / \mathrm{kg}$ & \\
\hline Diethylphthalate & & ND & & $330 . \mu \mathrm{g} / \mathrm{kg}$ & 2,4,5-Trichlorophenol & & ND & & 330. $\mu \mathrm{g} / \mathrm{kg}$ & \\
\hline 2,4-Dimethylphen & & ND & & 330. $\mu \mathrm{g} / \mathrm{kg}$ & 2,4,6-Trichlorophenol & & ND & $\mathbf{J}$ & 330. $\mu \mathrm{g} / \mathrm{kg}$ & \\
\hline
\end{tabular}

QUALITY CONTROL DATA:

\section{Surrogate}

2,4,6-Tribromophenol

2-Fluorobipheny]

2-Fluorophenol

Nitrobenzene-d5

p-Terphenyl-d14

Phenol-d6

\begin{tabular}{c} 
\% Recovery \\
\hline 49 \\
50 \\
44 \\
47 \\
66 \\
53
\end{tabular}

Acceptable Range

$19-122$

$30-115$

$25-121$

$23-120$

$18-137$

$24-113$

ND - Not Detected

This report shall not be reproduced except in full, without the written approval of the laboratory. 


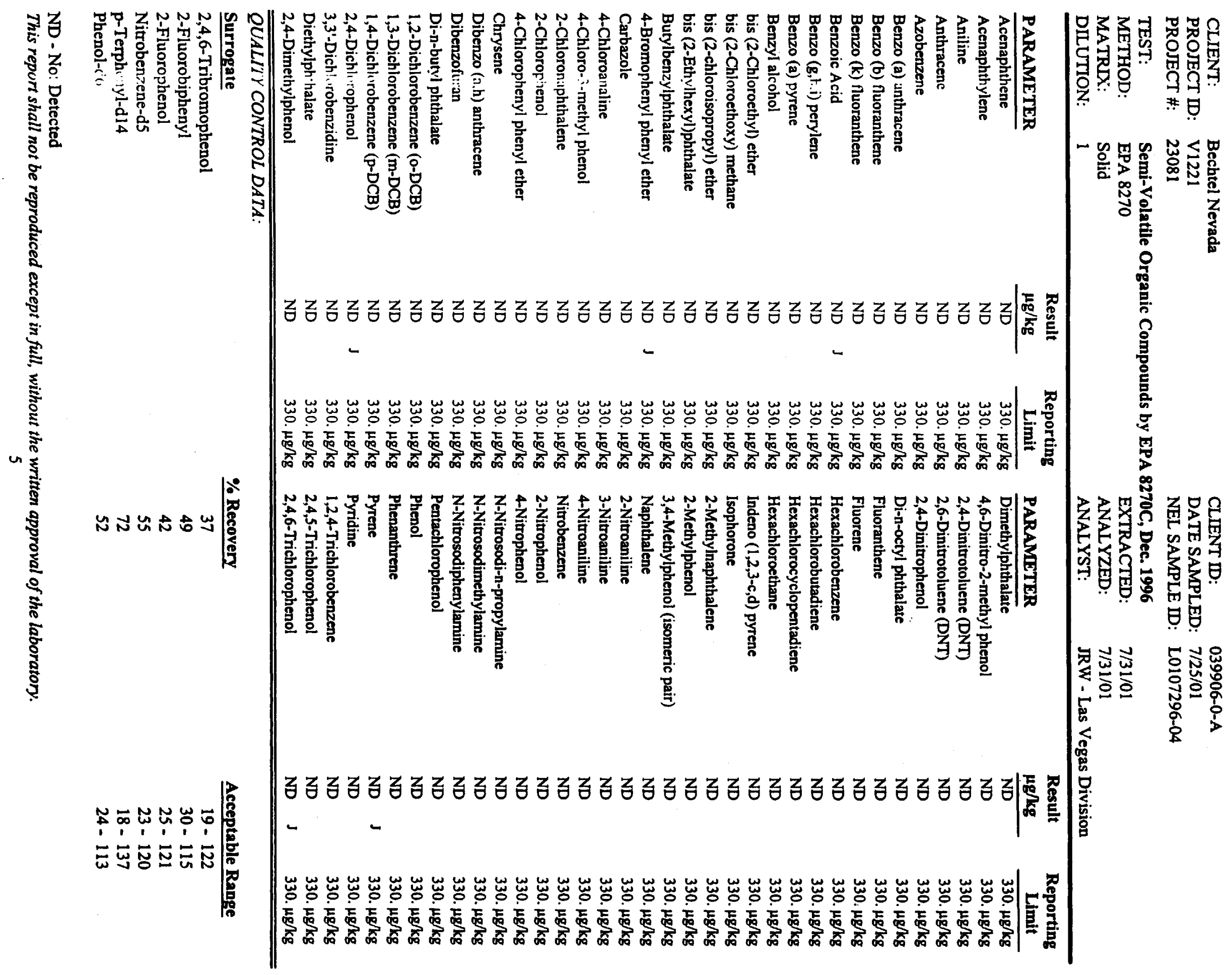




$\begin{array}{llll}\text { CLIENT: } & \text { Bechtel Nevada } & \text { CLIENT ID: } & \text { Method Blank } \\ \text { PROJECT D: } & \text { V1221 } & \text { DATE SAMPLED: NA } \\ \text { PROJECT \#: } & 23081 & \text { NEL SAMPLE DD: } 010730-8270 \text { S-BLK }\end{array}$

TEST:

METHOD:

Semi-Volatile Organic Compounds by EPA 8270C, Dec. 1996

MATR 8270

MATRX: Solid

\section{PARAMETER}

Acenaphthene

Acenaphthylene

Aniline

Anthracene

Azobenzene

Benzo (a) anthracene

Benzo (b) fluoranthene

Benzo (k) fluoranthene

Benzoic Acid

Benzo $(g, h, i)$ perylene

Benzo (a) pyrene

Benzyl al:ohol

bis (2-Chloroethyl) ether

bis (2-Chloroethoxy) methane

bis (2-chloroisopropyl) ether

bis (2-Ethy'lhexyl)phthalate

Butylbenzylphthalate

4-Bromophenyl phenyl ether

Carbazole

4-Chloroanaline

4-Chloro-3-methyl phenol

2-Chlorn: 1aphthalene

2-Chlore?henol

4-Chlorophenyl phenyl ether

Chrysene

Dibenzo (a.h) anthracene

Dibenzofiran

Di-n-butyl phthalate

1,2-Dichlorobenzene (O-DCB)

1,3-Dichlorobenzene (m-DCB)

1,4-Dichlorobenzene ( $\mathrm{p}-\mathrm{DCB})$

2,4-Dich 'nrophenol

3,3'-Dic' lorobenzidine
ANALYST:

EXTRACTED:

ANAL YZED:
JRW - Las Vegas Division

$7 / 31 / 01$

$7 / 30 / 01$

QUAL//Y CONTROL DATA:

\section{Surrow:ite}

2,4,6-7 hromophenol

2-Fluo siphenyl

2-Flun" "'henol

Nitroh'י'zene-d5

p-Terricienyl-d14

Phenn' 16

\begin{tabular}{|c|c|c|c|c|}
\hline $\begin{array}{l}\text { Result } \\
\mu \mathrm{g} / \mathrm{kg}\end{array}$ & $\begin{array}{l}\text { Reporting } \\
\text { Limit }\end{array}$ & PARAMETER & $\begin{array}{l}\text { Result } \\
\mu \mathrm{g} / \mathrm{kg}\end{array}$ & $\begin{array}{l}\text { Reporting } \\
\text { Limit }\end{array}$ \\
\hline ND & $330 \mu \mathrm{g} / \mathrm{kg}$ & Diethylphthalate & ND & $330 \mu \mathrm{g} / \mathrm{kg}$ \\
\hline ND & $330 \mu \mathrm{g} / \mathrm{kg}$ & 2,4-Dimethylphenol & ND & $330 \mu \mathrm{g} / \mathrm{kg}$ \\
\hline ND & $330 \mu \mathrm{g} / \mathrm{kg}$ & Dimethylphthalate & ND & $330 \mu \mathrm{g} / \mathrm{kg}$ \\
\hline ND & $330 \mu \mathrm{g} / \mathrm{kg}$ & 4,6-Dinitro-2-methyl phenol & ND & $330 \mu \mathrm{g} / \mathrm{kg}$ \\
\hline ND & $330 \mu \mathrm{g} / \mathrm{kg}$ & 2,4-Dinitrotoluene (DNT) & ND & $330 \mu \mathrm{g} / \mathrm{kg}$ \\
\hline ND & $330 \mu \mathrm{g} / \mathrm{kg}$ & 2,6-Dinitrotoluene (DNT) & ND & $330 \mu \mathrm{g} / \mathrm{kg}$ \\
\hline ND & $330 \mu \mathrm{g} / \mathrm{kg}$ & 2,4-Dinitrophenol & ND & $330 \mu \mathrm{g} / \mathrm{kg}$ \\
\hline ND & $330 \mu \mathrm{g} / \mathrm{kg}$ & Di-n-octyl phthalate & ND & $330 \mu \mathrm{g} / \mathrm{kg}$ \\
\hline ND & $330 \mu \mathrm{g} / \mathrm{kg}$ & Fluoranthene & ND & $330 \mu \mathrm{g} / \mathrm{kg}$ \\
\hline ND & $330 \mu \mathrm{g} / \mathrm{kg}$ & Fluorene & ND & $330 \mu \mathrm{g} / \mathrm{kg}$ \\
\hline ND & $330 \mu \mathrm{g} / \mathrm{kg}$ & Hexachlorobenzene & ND & $330 \mu \mathrm{g} / \mathrm{kg}$ \\
\hline ND & $330 \mu \mathrm{g} / \mathrm{kg}$ & Hexachlorobutadiene & ND & $330 \mu \mathrm{g} / \mathrm{kg}$ \\
\hline ND & $330 \mu \mathrm{g} / \mathrm{kg}$ & Hexachlorocyclopentadiene & ND & $330 \mu \mathrm{g} / \mathrm{kg}$ \\
\hline ND & $330 \mu \mathrm{g} / \mathrm{kg}$ & Hexachloroethane & ND & $330 \mu \mathrm{g} / \mathrm{kg}$ \\
\hline ND & $330 \mu \mathrm{g} / \mathrm{kg}$ & Indeno $(1,2,3-c, d)$ pyrene & ND & $330 \mu \mathrm{g} / \mathrm{kg}$ \\
\hline ND & $330 \mu \mathrm{g} / \mathrm{kg}$ & lsophorone & ND & $330 \mu \mathrm{g} / \mathrm{kg}$ \\
\hline ND & $330 \mu \mathrm{g} / \mathrm{kg}$ & 2-Methylnaphthalene & ND & $330 \mu \mathrm{g} / \mathrm{kg}$ \\
\hline ND & $330 \mu \mathrm{g} / \mathrm{kg}$ & 2-Methylphenol & ND & $330 \mu \mathrm{g} / \mathrm{kg}$ \\
\hline ND & $330 \mu \mathrm{g} / \mathrm{kg}$ & 3,4-Methylphenol (isomeric pair) & ND & $330 \mu \mathrm{g} / \mathrm{kg}$ \\
\hline ND & $330 \mu \mathrm{g} / \mathrm{kg}$ & Naphthalene & ND & $330 \mu \mathrm{g} / \mathrm{kg}$ \\
\hline ND & $330 \mu \mathrm{g} / \mathrm{kg}$ & 2-Nitroaniline & ND & $330 \mu \mathrm{g} / \mathrm{kg}$ \\
\hline ND & $330 \mu \mathrm{g} / \mathrm{kg}$ & 3-Nitroaniline & ND & $330 \mu \mathrm{g} / \mathrm{kg}$ \\
\hline ND & $330 \mu \mathrm{g} / \mathrm{kg}$ & 4-Nitroaniline & ND & $330 \mu \mathrm{g} / \mathrm{kg}$ \\
\hline ND & $330 \mu \mathrm{g} / \mathrm{kg}$ & Nitrobenzene & ND & $330 \mu \mathrm{g} / \mathrm{kg}$ \\
\hline ND & $330 \mu \mathrm{g} / \mathrm{kg}$ & 2-Nitrophenol & ND & $330 \mu \mathrm{g} / \mathrm{kg}$ \\
\hline ND & $330 \mu \mathrm{g} / \mathrm{kg}$ & 4-Nitrophenol & ND & $330 \mu \mathrm{g} / \mathrm{kg}$ \\
\hline ND & $330 \mu \mathrm{g} / \mathrm{kg}$ & N-Nitrosodi-n-propylamine & ND & $330 \mu \mathrm{g} / \mathrm{kg}$ \\
\hline ND & $330 \mu \mathrm{g} / \mathrm{kg}$ & N-Nitrosodimethylamine & ND & $330 \mu \mathrm{g} / \mathrm{kg}$ \\
\hline ND & $330 \mu \mathrm{g} / \mathrm{kg}$ & N-Nitrosodiphenylamine & ND & $330 \mu \mathrm{g} / \mathrm{kg}$ \\
\hline ND & $330 \mu \mathrm{g} / \mathrm{kg}$ & Pentachlorophenol & ND & $330 \mu \mathrm{g} / \mathrm{kg}$ \\
\hline ND & $330 \mu \mathrm{g} / \mathrm{kg}$ & Phenol & ND & $330 \mu \mathrm{g} / \mathrm{kg}$ \\
\hline ND & $330 \mu \mathrm{g} / \mathrm{kg}$ & Phenanthrene & ND & $330 \mu \mathrm{g} / \mathrm{kg}$ \\
\hline ND & $330 \mu \mathrm{g} / \mathrm{kg}$ & Pyrene & ND & $330 \mu \mathrm{g} / \mathrm{kg}$ \\
\hline
\end{tabular}

\begin{tabular}{l} 
Acceptable Range \\
\hline $19-122$ \\
$30-115$ \\
$25-121$ \\
$23-120$ \\
$18-137$ \\
$24-113$
\end{tabular}

ND - $\Lambda$, Detected

This $r_{\mathrm{L}} \cdot \mathrm{r}_{\mathrm{t}}$ shall not be reproduced except in full, without the written approval of the laboratory. 
- CLIENT: Bechtel Nevada

PROJECT ID: V1221

PROJECT \#: 23081
CLIENT ID: $\quad$ 039903-0-A

DATE SAMPLED: 7/24/01

NEL SAMPLE ID: L0107296-01

TEST: $\quad$ Total Extractable Petroleum Hydrocarbons Fuel Finger Print by EPA Method 8015M, July 1992

METY:OD: $\quad$ EPA 8015M

MATIL: $\quad$ Solid ANALYST: $\quad$ CCS - Las Vegas Division

DILUTION: 1 EXTRACTED: $\quad 8 / 3 / 01$ ANALYZED: $\quad 8 / 1 / 01$

\section{PARA METER}

- Gasoli?: Range (C8-C12)
Diesel Range (C12-C22)

Gasoline Range (C8-C12)
Diesel Range (C12-C22)

\begin{tabular}{l} 
Result \\
\hline ND \\
ND \\
ND \\
ND
\end{tabular}

Reporting Limit Oil Range (C22-C34)

ND 10. $\mathrm{mg} / \mathrm{kg}$ 10. $\mathrm{mg} / \mathrm{kg}$ 50. $\mathrm{mg} / \mathrm{kg}$

Total

\section{\% Recovery}

Surro::-ate

Octar. sane

74
ND - $\therefore$ ot Detected

This riport shall not be reproduced except in full, without the written approval of the laboratory.

\section{Acceptable Range}

$54-130$ 


$\begin{array}{lll}\text { CLIE'T: } & \text { Bechtel Nevada } & \text { CLIENT DD: } \quad \text { 039901-0-A } \\ \text { PROJ_CT ID: } & \text { V1221 } & \text { DATE SAMPLED: } 7 / 25 / 01 \\ \text { PROJECT \#: } & 23081 & \text { NEL SAMPLE ID: L0107296-02 }\end{array}$

TEST: Total Extractable Petroleum Hydrocarbons Fuel Finger Print by EPA Method 8015M, July 1992

MET: JD: EPA 8015M ANALYST: CCS - Las Vegas Division

MAT.IX: Solid $\quad$ EXTRACTED: $8 / 3 / 01$

DILLTION: 1 ANALYZED: $8 / 1 / 01$

\begin{tabular}{lll}
\hline PAR.1.METER & Result & Reporting \\
\cline { 2 - 2 } & $\mathrm{ND}$ & $\frac{\text { Limit }}{10 . \mathrm{mg} / \mathrm{kg}}$ \\
Diesc' : ange (C12-C22) & $\mathrm{ND}$ & $10 . \mathrm{mg} / \mathrm{kg}$ \\
Oil R:!'ge (C22-C34) & $\mathrm{ND}$ & $50 . \mathrm{mg} / \mathrm{kg}$ \\
Total & $\mathrm{ND}$ & $10 . \mathrm{mg} / \mathrm{kg}$ \\
\hline \hline
\end{tabular}

QUA: TY CONTROL DATA:

Surr. : ite

Octas.: ane

\% Recovery

65
Acceptable Range

$54-130$

ND - it Detected

This : 'ort shall not be reproduced except in full, without the written approval of the laboratory. 
CLIE T: Bechtel Nevada

PROJ :CT ID: V1221

PROJ!CT \#: 23081

TEST

MET： JD:

MATJ.IX:

DILUIION:

EPA 8015M

Solid

Total Extractable Petroleum Hydrocarbons Fuel Finger Print by EPA Method 8015M, July 1992

CLIENT ID: $\quad$ 039901-0-B

DATE SAMPLED: 7/25/01

NEL SAMPLE ID: L0107296-03

PAR : METER

Gasn': :Range (C8-C12)

Dics: :ange (C12-C22)

Oil R: : e (C22-C34) ANALYST: CCS - Las Vegas Division EXTRACTED: $8 / 3 / 01$

Total ANALYZED:

$8 / 2 / 01$

$\overline{\overline{Q U A}}$ TY CONTROL DATA:

Surr: inte

Octac ane

$\%$ Recovery

113
Reporting

Limit

10. $\mathrm{mg} / \mathrm{kg}$

10. $\mathrm{mg} / \mathrm{kg}$

50. $\mathrm{mg} / \mathrm{kg}$

10. $\mathrm{mg} / \mathrm{kg}$

ND

ND

ND

Acceptable Range

$54-130$

ND - ot Detected

This, 'ort shall not be reproduced except in full, without the written approval of the laboratory. 


\begin{tabular}{|c|c|c|c|}
\hline $\begin{array}{l}\text { CLII } \mathrm{r}: \\
\text { PRO: CT ID: } \\
\text { PRO : CT \#: }\end{array}$ & $\begin{array}{l}\text { Bechtel Nevada } \\
\text { V1221 } \\
23081\end{array}$ & $\begin{array}{l}\text { CLIENT ID: } \\
\text { DATE SAMPLED: } \\
\text { NEL SAMPLE ID: }\end{array}$ & $\begin{array}{l}\text { Method Blank } \\
\text { NA } \\
\text { 010731TPHS/FP-BLK }\end{array}$ \\
\hline
\end{tabular}

TES: $\quad$ Total Extractable Petroleum Hydrocarbons Fuel Finger Print by EPA Method 8015M, July 1992

ME: :OD: EPA 8015M ANALYST: CCS - Las Vegas Division

MATSIX: Solid EXTRACTED: 8/3/01

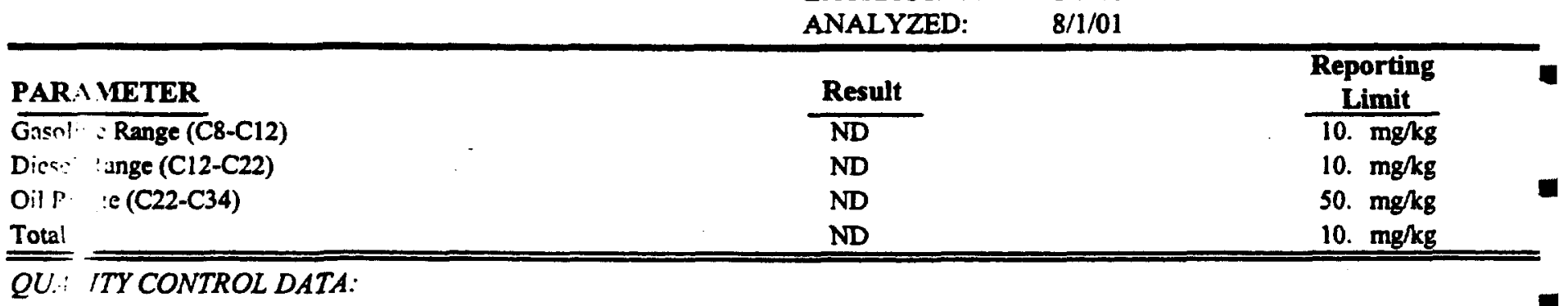

Surr. 迹e

Octacusane

\% Recovery

72
Acceptable Range

$54-130$

Ni) . ot Detected

T.i.. Iort shall not be reproduced except in full, without the written approval of the laboratory.

$\mathrm{CLI}$ T: Bechtel Nevada

CLIENT ID: $\quad$ Method Blank

PR( ' CT ID: V1221

DATE SAMPLED: NA

PRC, CT \#: 23081

NEL SAMPLE ID: 010801TPHS/FP-BLK

Tis, :

Total Ertractable Petroleum Hydrocarbons Fuel Finger Print by EPA Method 8015M, July 1992

ME11HOD: $\quad$ EPA 8015M

MATRIX: Solid

ANALYST: $\quad$ CCS - Las Vegas Division

EXTRACTED: $\quad 8 / 3 / 01$

ANALYZED: $\quad 8 / 2 / 01$

\begin{tabular}{|c|c|c|c|}
\hline$P \cup !$ & IIETER & Result & $\begin{array}{c}\text { Reporting } \\
\text { Limit } \\
\end{array}$ \\
\hline $\bar{c}$ & $\overline{\text { Range (C } 8-\mathrm{C} 12)}$ & $\mathrm{ND}$ & 10. $\mathrm{mg} / \mathrm{kg}$ \\
\hline$\Gamma$ & ange (C12-C22) & ND & 10. $\mathrm{mg} / \mathrm{kg}$ \\
\hline ( $1:$ & $\therefore(\mathrm{C} 22-\mathrm{C} 34)$ & ND & 50. $\mathrm{mg} / \mathrm{kg}$ \\
\hline Iut: & & ND & 10. $\mathrm{mg} / \mathrm{kg}$ \\
\hline$\varepsilon$ & YY CONT & & \\
\hline$\frac{\text { Surnes }}{\text { Ocin }}$ & $\frac{\text { gate }}{\text { osane }}$ & $\frac{\text { Recovery }}{122}$ & $\frac{\text { Acceptable RaI }}{54-130}$ \\
\hline
\end{tabular}

1. ut Detected

I port shall not be reproduced except in full, without the written approval of the laboratory. 


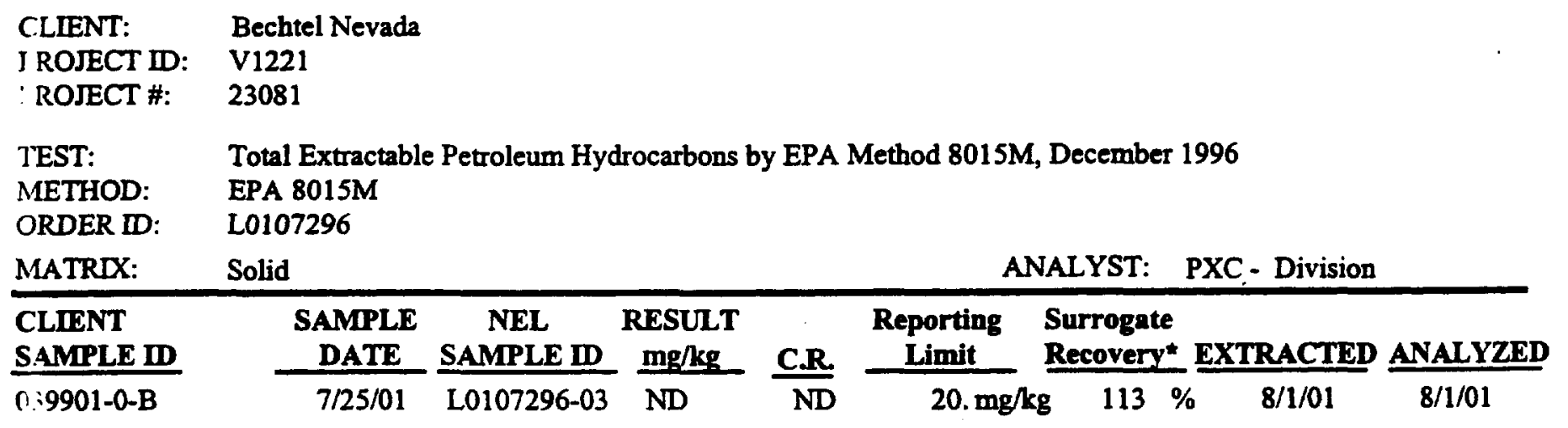

\section{C.R.: Carbon Range}

(UUALITY CONTROL DATA (Total for Diesel Range):

Simple ID

Blank, 010801TP -BLK

I CS, 010801TPHSI-LCS

1.CSD, 010801TPHS1-LCSD

'IS, 010801TPHS1-MS

$\therefore$ ISD, 010801TPHS1-MSD

Surrogate used was Octacosane, acceptance limits 55-130\%.

\begin{tabular}{|c|c|c|c|c|c|}
\hline Result & Acce & ble Range S & ate I & lecovery ${ }^{\star}$ & Sample Numbe \\
\hline ND & $<$ & $20 \mathrm{mg} / \mathrm{kg}$ & 122 & $\%$ & NA \\
\hline 69 & 54 & $-91 \%$ & 119 & $\%$ & NA \\
\hline 75 & 54 & $-93 \%$ & 99 & $\%$ & NA \\
\hline $70 \%$ & 34 & $-110 \%$ & 108 & $\%$ & L0107296-03 \\
\hline $71 \%$ & 34 & $-110 \%$ & 110 & $\%$ & L0107296-03 \\
\hline
\end{tabular}

ND - Not Detected

7h is report shall not be reproduced except in full, without the written approval of the laboratory. 


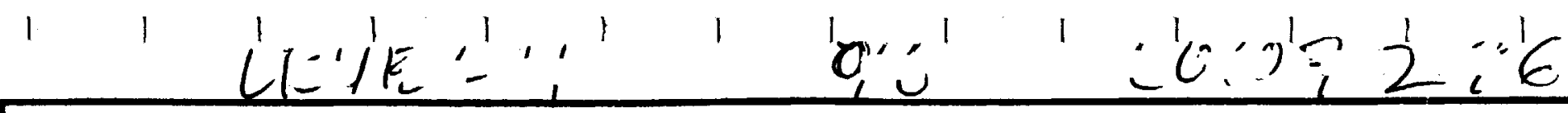

Bebhtel hevada ANALYTICAL SERVICES LABORATORY

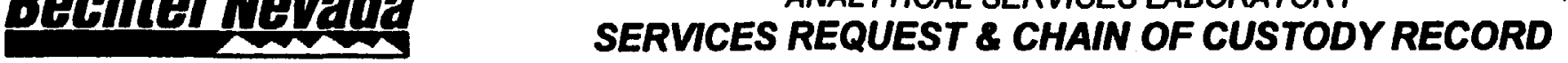

Page $L$ of 1

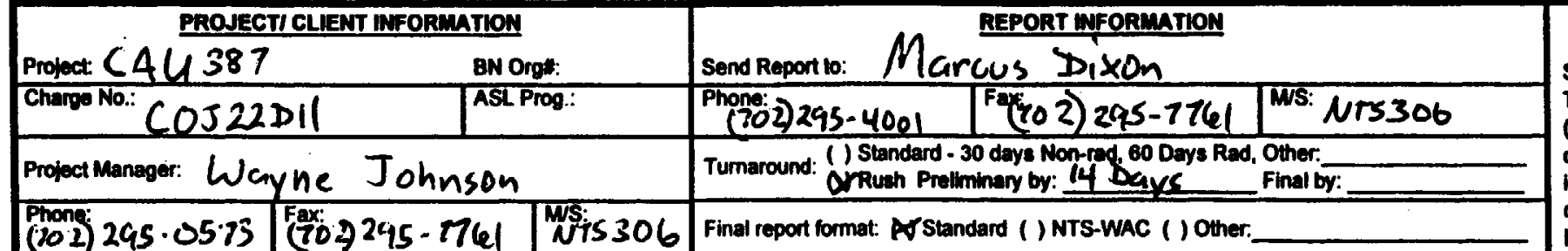

\section{SALPLEINFORLATION}

Sampling Site: $\mathrm{CAU} 387$

The samples submitted contain (ctrock);

(1) Harandous (1) Radiosetive (Druntoun contamination. I known, altach a brier narrative summenty contifying contaminants. This information witt ensure compliance with applicable regulations and allow for the sale handling of the sample meterials.

DATE The matrix

$032039901-0-B$

0.7 \begin{tabular}{l|l}
3 & $039906-0-A$
\end{tabular} LAB USE OMLY

Red SGD: Non-Rad SDG: $1 / 1221$

Cliont Sorvices Representative:

Non-Rad Packet:

Will these analyees be pertormed under a signed SOW? () YES ( ) NO

If not, ldentify the variation

\begin{tabular}{|c|c|c|c|c|}
\hline $\mathbf{T}$ & \multirow{2}{*}{ DIOESCRIPTION } & \multicolumn{2}{|c|}{ SAMPLINO } & \multirow{2}{*}{ MATRIX } \\
\hline $\mathbf{E}$ & DATE & TIME & \\
\hline
\end{tabular}

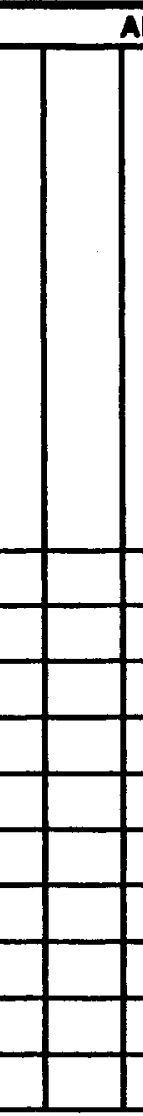

SALPPLE RECEIPT MFOREMTIOY.

Aro all sample containers received intact $M\left(Y_{\text {Yes }}(\right.$ ) No

Comments:

\section{Do the labels arree with this form?}

Comments:

SYes ( ) No

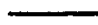

Was a Malerial Clearance Tag submitted? (1) Y Yes ( ) No Comments:

1/24la $13: 46$ so: $\quad x \quad x$ 7) $23 i_{11}$ 13:10 $50, i l|x| x$ $7 / 25 / a$ 13:15 soil $x \quad x$

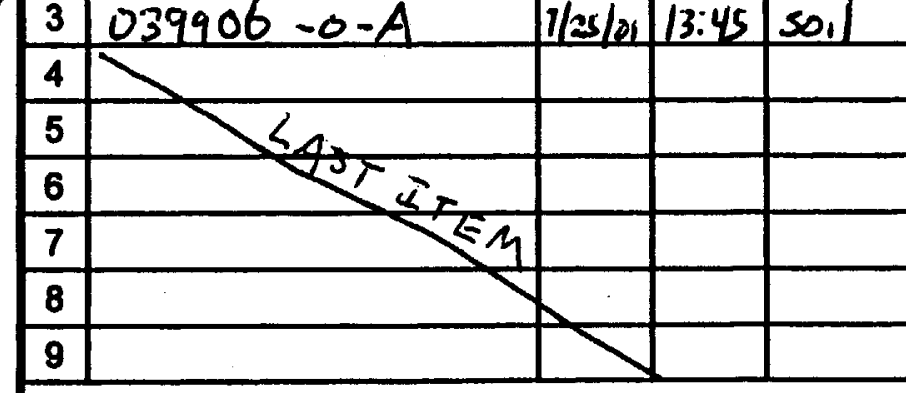

Transfor of semples submitted for analyses

\begin{tabular}{|l|l|l}
\hline Semplea/Relmquishod (Signature/Organization) & DATE / TME & Recelved by (Signature/Organization)
\end{tabular} Maram Dime

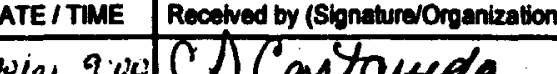
Complete for samples shipped to an OFF-SITE Subcontract Laboratory NEL

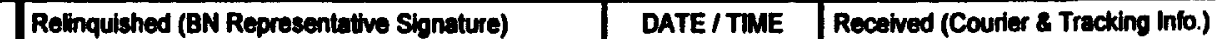

\begin{tabular}{|l|l|l|}
\hline & & \\
\hline & & \\
\hline & & \\
\hline & & \\
\hline
\end{tabular}
CDCastounda Reynglished (Coyiler \& Tracting info.)

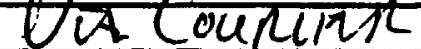




$\begin{array}{ll}\text { CLIENT: } & \text { Bechtel Nevada } \\ & \text { P.O. Box 98521, M/S NTS273 } \\ & \text { Las Vegas, NV 89193-8521 } \\ \text { ATTN: } & \text { Ted Redding }\end{array}$

PROJECT NAME: $\quad$ V1234

PROJECT NUMBER: 23081
NEL ORDER ID: L0108079

Attached are the analytical results for samples in support of the above referenced project.

Samples submitted for this project were not sampled by NEL Laboratories. Samples were received by NEL in good condition, under chain of custody on 8/9/01.

Should you have any questions or comments, please feel free to contact our Client Services department at (702) 657-1010.

\section{Some results have been flagged as follows:}

J - This concentration should be considered an estimate due laboratory control sample failure.

ת - The batch MS and/or MSD were outside acceptance limits. The batch LCS was acceptable.

Some $Q A$ results have been flagged as follows:

J - This concentration should be considered an estimate due laboratory control sample failure.

J1 - The batch MS and/or MSD were outside acceptance limits. The batch LCS was acceptable.
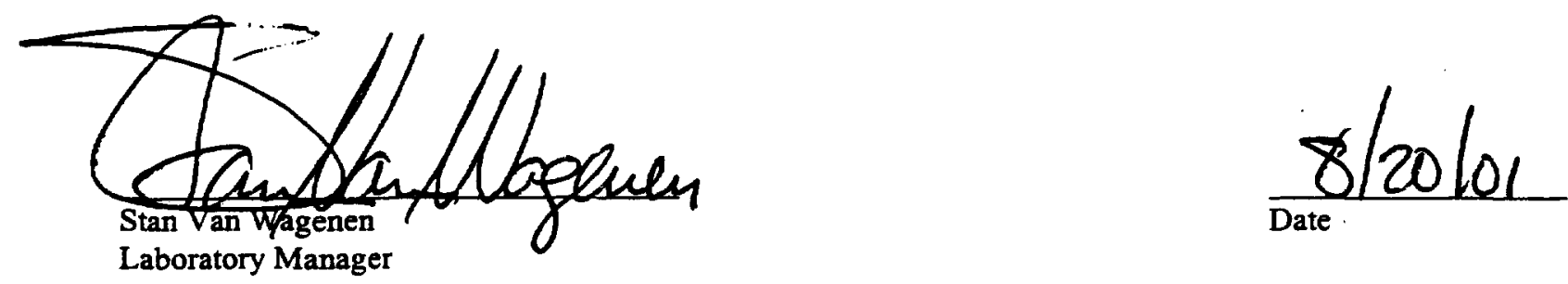

\section{CERTIFICATIONS:}

\begin{tabular}{llll}
\hline & Reno & Las Vegas & S. Califomia \\
\cline { 2 - 4 } $\begin{array}{l}\text { Arizona } \\
\text { California }\end{array}$ & 170520 & AZ0518 & AZ0605 \\
$\begin{array}{l}\text { US Army Corps } \\
\text { of Engineers }\end{array}$ & Certified & Certified & 2264 \\
& & &
\end{tabular}

\begin{tabular}{llll} 
& Reno & Las Vegas & S. California \\
\cline { 2 - 4 } Idaho & Certified & Certified & \\
Montana & Certified & Certified & \\
Nevada & NV033 & NV052 & CA084 \\
L.A.C.S.D. & & & 10228
\end{tabular}




\begin{tabular}{|c|c|c|c|c|c|c|c|c|c|}
\hline $\begin{array}{l}\text { CCLIENT: } \\
\text { PROJECT ID: } \\
\text { PROJECT \#: }\end{array}$ & $\begin{array}{l}\text { Bechtel Nevada } \\
\text { V1234 } \\
23081\end{array}$ & & & & $\begin{array}{l}\text { CLIENT ID: } \\
\text { DATE SAMPLED: } \\
\text { NEL SAMPLE ID: }\end{array}$ & $\begin{array}{l}079903-0-A \\
8 / 8 / 01 \\
\text { L0108079-01 }\end{array}$ & & & \\
\hline $\begin{array}{l}\text { TEST: } \\
\text { METHOD: } \\
\text { MATRIX: } \\
\text { DILUTION: }\end{array}$ & $\begin{array}{l}\text { Semi-Volatile } C \\
\text { EPA } 8270 \\
\text { Solid } \\
1 \\
\end{array}$ & anic Co & mpo & nds by EPA & $\begin{array}{l}\text { 270C, Dec. } 1996 \\
\text { EXTRACTED: } \\
\text { ANALYZED: } \\
\text { ANALYST: }\end{array}$ & $\begin{array}{l}8 / 12 / 01 \\
8 / 15 / 01 \\
\text { JRW - Las Vega }\end{array}$ & s Divis & & \\
\hline PARAMETER & & $\begin{array}{l}\text { Result } \\
\mu \mathrm{g} / \mathrm{kg}\end{array}$ & & $\begin{array}{c}\text { Reporting } \\
\text { Limit }\end{array}$ & PARAMETER & & $\begin{array}{l}\text { Resul } \\
\mu \mathrm{g} / \mathrm{kg}\end{array}$ & & $\begin{array}{c}\text { Reporting } \\
\text { Limit }\end{array}$ \\
\hline Acenaphthene & & ND & & $330 . \mu \mathrm{g} / \mathrm{kg}$ & $\overline{\text { Dimethylphthaiate }}$ & & $\overline{\mathrm{ND}}$ & & $330 . \mu \mathrm{g} / \mathrm{kg}$ \\
\hline Acenaphthylene & & ND & & $330 . \mu \mathrm{g} / \mathrm{kg}$ & 4,6-Dinitro-2-methyl & shenol & ND & $\mathbf{J} \mathbf{1}$ & $330 . \mu \mathrm{g} / \mathrm{kg}$ \\
\hline Aniline & & ND & & 330. $\mu \mathrm{g} / \mathrm{kg}$ & 2,4-Dinitrotoluene (D) & vT) & ND & & 330. $\mu \mathrm{g} / \mathrm{kg}$ \\
\hline Anthracene & & ND & & $330 . \mu \mathrm{g} / \mathrm{kg}$ & 2,6-Dinitrotoluene (D) & NT) & ND & & $330 . \mu \mathrm{g} / \mathrm{kg}$ \\
\hline Azobenzene & & ND & & $330 . \mu \mathrm{g} / \mathrm{kg}$ & 2,4-Dinitrophenol & & ND & J & $330 . \mu \mathrm{g} / \mathrm{kg}=$ \\
\hline Benzo (a) anthrace & & ND & & $330 . \mu \mathrm{g} / \mathrm{kg}$ & Di-n-octyl phthalate & & ND & & $330 . \mu \mathrm{g} / \mathrm{kg}$ \\
\hline Benzo (b) fluorant & thene & ND & & $330 . \mu g / k g$ & Fluoranthene & & ND & & 330. $\mu \mathrm{g} / \mathrm{kg}$ \\
\hline Benzo (k) fluorant & thene & ND & & $330 . \mu \mathrm{g} / \mathrm{kg}$ & Fluorene & & ND & & $330 . \mu \mathrm{g} / \mathrm{kg}$ \\
\hline Benzoic Acid & & ND & $J$ & $330 . \mu \mathrm{g} / \mathrm{kg}$ & Hexachlorobenzene & & ND & & 330. $\mu \mathrm{g} / \mathrm{kg}$ \\
\hline Benzo $(g, h, i)$ peryl & lene & ND & & $330 . \mu \mathrm{g} / \mathrm{kg}$ & Hexachlorobutadiene & & ND & & 330. $\mu \mathrm{g} / \mathrm{kg}$ \\
\hline Benzo (a) pyrene & & ND & & 330. $\mu \mathrm{g} / \mathrm{kg}$ & Hexachlorocyclopenta & diene & ND & $\mathrm{Jl}$ & 330. $\mu \mathrm{g} / \mathrm{kg}$ \\
\hline Benzyl alcohol & & ND & & $330 . \mu \mathrm{g} / \mathrm{kg}$ & Hexachloroethane & & ND & & 330. $\mu \mathrm{g} / \mathrm{kg}$ \\
\hline bis (2-Chloroethyl) & ) ether & ND & & $330 . \mu \mathrm{g} / \mathrm{kg}$ & Indeno $(1,2,3-c, d)$ pyre & & ND & & 330. $\mu \mathrm{g} / \mathrm{kg}$ \\
\hline bis (2-Chloroethox & xy) methane & ND & & 330. $\mu \mathrm{g} / \mathrm{kg}$ & Isophorone & & ND & & 330. $\mu \mathrm{g} / \mathrm{kg}$ \\
\hline bis (2-chloroisoprc & opyl) ether & ND & $\pi$ & 330. $\mu \mathrm{g} / \mathrm{kg}$ & 2-Methylnaphthalene & & ND & & 330. $\mu \mathrm{g} / \mathrm{kg}=$ \\
\hline bis (2-Ethylhexyl) & phthalate & ND & & 330. $\mu \mathrm{g} / \mathrm{kg}$ & 2-Methylphenol & & ND & & 330. $\mathrm{\mu g} / \mathrm{kg}$ \\
\hline Butylbenzylphthal & & ND & & 330. $\mu \mathrm{g} / \mathrm{kg}$ & 3,4-Methylphenol (iso & meric pair) & ND & & $330 . \mu \mathrm{g} / \mathrm{kg}$ \\
\hline 4-Bromophenyl ph & henyl ether & ND & & 330. $\mu \mathrm{g} / \mathrm{kg}$ & Naphthalene & & ND & & 330. $\mu \mathrm{g} / \mathrm{kg}$ \\
\hline Carbazole & & ND & & $330 . \mu \mathrm{g} / \mathrm{kg}$ & 2-Nitroaniline & & ND & & $339 . \mu \mathrm{g} / \mathrm{kg}$ \\
\hline 4-Chloroanaline & & ND & & 330. $\mu \mathrm{g} / \mathrm{kg}$ & 3-Nitroaniline & & ND & & $330 . \mu \mathrm{g} / \mathrm{kg}$ \\
\hline 4-Chloro-3-methy & I phenol & ND & & $330 . \mu \mathrm{g} / \mathrm{kg}$ & 4-Nitroaniline & & ND & & 330. $\mu \mathrm{g} / \mathrm{kg}$ \\
\hline 2-Chloronaphthale & & ND & & 330. $\mu \mathrm{g} / \mathrm{kg}$ & Nitrobenzene & & ND & & 330. $\mu \mathrm{g} / \mathrm{kg}=$ \\
\hline 2-Chlorophenol & & ND & & $330 . \mu \mathrm{g} / \mathrm{kg}$ & 2-Nitrophenol & & ND & л & $330 . \mu \mathrm{g} / \mathrm{kg}$ \\
\hline 4-Chlorophenyl ph & enyl ether & ND & & $330 . \mu \mathrm{g} / \mathrm{kg}$ & 4-Nitrophenol & & ND & & $330 . \mu \mathrm{g} / \mathrm{kg}$ \\
\hline Chrysene & & ND & & $330 . \mu \mathrm{g} / \mathrm{kg}$ & N-Nitrosodi-n-propyla & mine & ND & & $330 . \mu \mathrm{g} / \mathrm{kg}$ \\
\hline Dibenzo $(a, h)$ anth & Iracene & ND & & $330 . \mu \mathrm{g} / \mathrm{kg}$ & N-Nitrosodimethylami & & ND & & 330. $\mu \mathrm{g} / \mathrm{kg}$ \\
\hline Dibenzofuran & & ND & & $330 . \mu \mathrm{g} / \mathrm{kg}$ & N-Nitrosodiphenylami & & ND & & $330 . \mu \mathrm{g} / \mathrm{kg}$ \\
\hline Di-n-butyl phthala & & ND & $\pi$ & 330. $\mu \mathrm{g} / \mathrm{kg}$ & Pentachlorophenol & & ND & & 330. $\mu \mathrm{g} / \mathrm{kg}$ \\
\hline 1,2-Dichlorobenze & ne $(0-D C B)$ & ND & & 330. $\mu \mathrm{g} / \mathrm{kg}$ & Phenol & & ND & & 330. $\mu \mathrm{g} / \mathrm{kg}$ \\
\hline 1,3-Dichlorobenze & ne (m-DCB) & ND & & $330 . \mu \mathrm{g} / \mathrm{kg}$ & Phenanthrene & & ND & & 330. $\mu \mathrm{g} / \mathrm{kg}$ \\
\hline 1,4-Dichlorobenze & ne (p-DCB) & ND & & 330. $\mu \mathrm{g} / \mathrm{kg}$ & Pyrene & & ND & лI & 330. $\mu \mathrm{g} / \mathrm{kg}$ \\
\hline 2,4-Dichlorophenc & & ND & & $330 . \mu \mathrm{g} / \mathrm{kg}$ & Pyridine & & ND & & $330 . \mu \mathrm{g} / \mathrm{kg}$ \\
\hline 3,3'-Dichlorobenzi & idine & ND & $\pi$ & 330. $\mu \mathrm{g} / \mathrm{kg}$ & 1,2,4-Trichlorobenzen & & ND & & 330. $\mu \mathrm{g} / \mathrm{kg}$ \\
\hline Diethylphthalate & & ND & & 330. $\mu \mathrm{g} / \mathrm{kg}$ & 2,4,5-Trichlorophenol & & ND & & 330. $\mu \mathrm{g} / \mathrm{kg}$ \\
\hline 2,4-Dimethylphen & & ND & & $330 . \mu \mathrm{g} / \mathrm{kg}$ & 2,4,6-Trichlorophenol & & ND & & $330 . \mu \mathrm{g} / \mathrm{kg}$ \\
\hline
\end{tabular}

QUALITY CONTROL DATA:

\section{Surrogate}

2,4,6-Tribromophenol

2-Fluorobiphenyl

2-Fluorophenol

Nitrobenzene-d5

p-Terphenyl-d14

Phenol-d6

\section{$\%$ Recovery}

90

70

73

65

82

77
Acceptable Range

$19-122$

$30-115$

$25-121$

$23-120$

$18-137$

$24-113$

ND - Not Detected

This report shall not be reproduced except in full, without the written approval of the laboratory. 


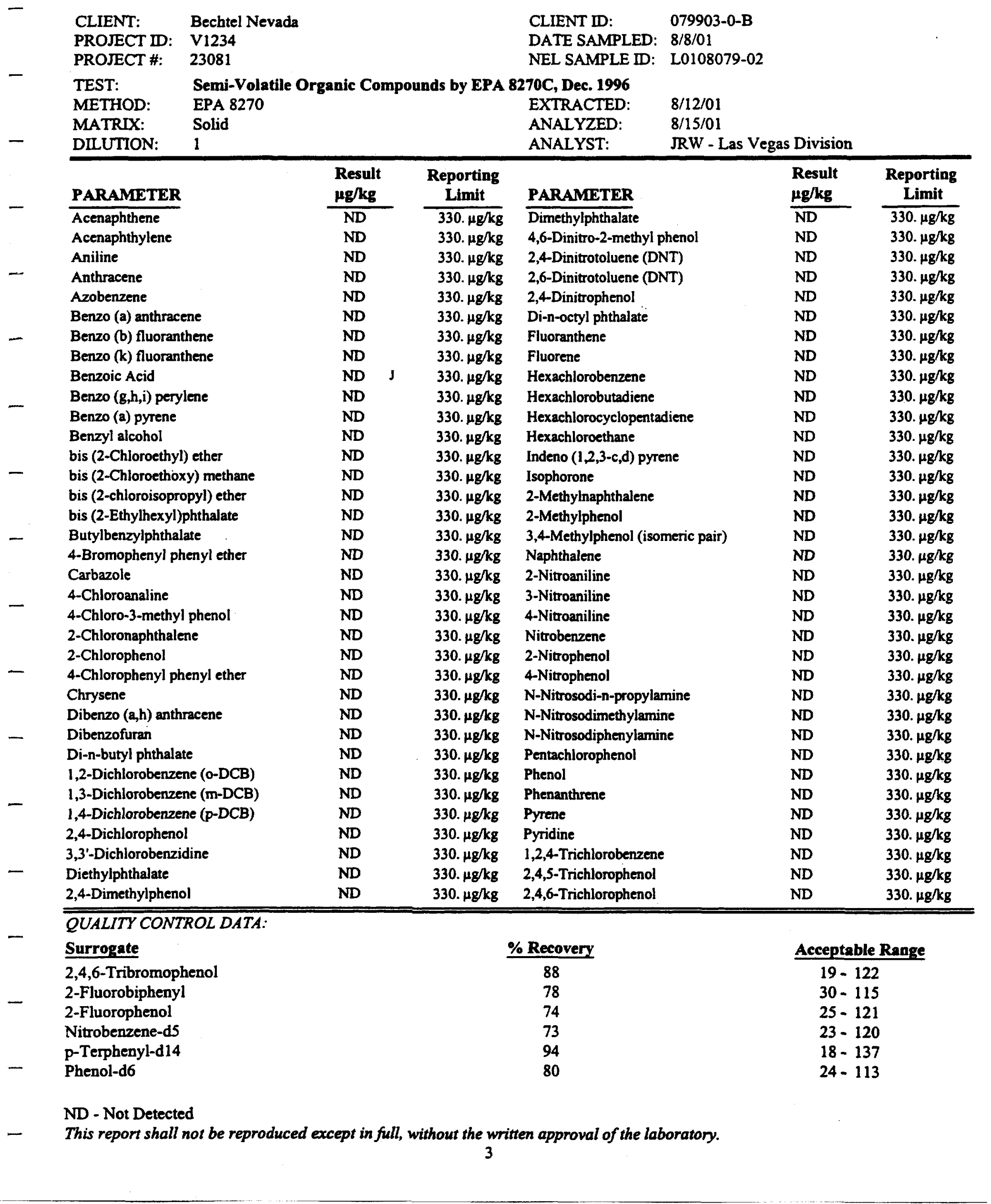




\begin{tabular}{|c|c|c|c|c|c|c|c|c|}
\hline $\begin{array}{l}\text { CLIENT: } \\
\text { PROJECT D: } \\
\text { PROJECT \#: }\end{array}$ & $\begin{array}{l}\text { Bechtel Nevada } \\
\text { V1234 } \\
23081\end{array}$ & & & & $\begin{array}{l}\text { CLIENT ID: } \\
\text { DATE SAMPLED: } \\
\text { NEL SAMPLE W: }\end{array}$ & \multicolumn{2}{|c|}{$\begin{array}{l}079903-0-C \\
8 / 8 / 01 \\
\text { L0108079-03 }\end{array}$} & \\
\hline $\begin{array}{l}\text { TEST: } \\
\text { METHOD: } \\
\text { MATRDX: } \\
\text { DILUTION: }\end{array}$ & \multicolumn{4}{|l|}{$\begin{array}{l}\text { EPA } 8270 \\
\text { Solid } \\
1\end{array}$} & $\begin{array}{l}\text { 270C, Dec. } 1996 \\
\text { EXTRACTED: } \\
\text { ANALYZED: } \\
\text { ANALYST: }\end{array}$ & \multicolumn{3}{|c|}{$\begin{array}{l}8 / 12 / 01 \\
8 / 15 / 01 \\
\text { JRW - Las Vegas Division }\end{array}$} \\
\hline PARAMETER & & $\begin{array}{l}\text { Result } \\
\mu g / k g\end{array}$ & & $\begin{array}{c}\text { Reporting } \\
\text { Limit }\end{array}$ & PARAMETER & & $\begin{array}{l}\text { Result } \\
\mu \mathrm{g} / \mathbf{k g}\end{array}$ & $\begin{array}{c}\text { Reporting } \\
\text { Limit }\end{array}$ \\
\hline Acenaphthene & & ND & & 330. $\mu \mathrm{g} / \mathrm{kg}$ & $\overline{\text { Dimethylphthalate }}$ & & ND & $330 . \mu \mathrm{g} / \mathrm{kg}$ \\
\hline Acenaphthylene & & ND & & 330. $\mu \mathrm{g} / \mathrm{kg}$ & 4,6-Dinitro-2-methyl p & henol & ND & 330. $\mu \mathrm{g} / \mathrm{kg}$ \\
\hline Aniline & & ND & & $330 . \mu g / k g$ & 2,4-Dinitrotoluene (DI & งT) & ND & 330. $\mu \mathrm{g} / \mathrm{kg}$ \\
\hline Anthracene & & ND & & 330. $\mu \mathrm{g} / \mathrm{kg}$ & 2,6-Dinitrotoluene (DI & VT) & ND & 330. $\mu \mathrm{g} / \mathrm{kg}$ \\
\hline Azobenzene & & ND & & $330 . \mu \mathrm{g} / \mathrm{kg}$ & 2,4-Dinitrophenol & & ND & $330 . \mu \mathrm{g} / \mathrm{kg}$ \\
\hline Benzo (a) anthrace & ene & ND & & 330. $\mu \mathrm{g} / \mathrm{kg}$ & Di-n-octyl phthalate & & ND & 330. $\mu \mathrm{g} / \mathrm{kg}$ \\
\hline Benzo (b) fluorant & thene & ND & & 330. $\mu \mathrm{g} / \mathrm{kg}$ & Fluoranthene & & ND & $330 . \mu \mathrm{g} / \mathrm{kg}$ \\
\hline Benzo (k) fluorant & thene & ND & & $330 . \mu \mathrm{g} / \mathrm{kg}$ & Fluorene & & ND & 330. $\mu \mathrm{g} / \mathrm{kg}$ \\
\hline Benzoic Acid & & ND & $J$ & 330. $\mathrm{\mu g} / \mathrm{kg}$ & Hexachlorobenzene & & ND & 330. $\mu \mathrm{g} / \mathrm{kg}$ \\
\hline Benzo $(g, h, i)$ pery & lene & ND & & $330 . \mu \mathrm{g} / \mathrm{kg}$ & Hexachlorobutadiene & & ND & 330. $\mu \mathrm{g} / \mathrm{kg}$ \\
\hline Benzo (a) pyrene & & ND & & 330. $\mu \mathrm{g} / \mathrm{kg}$ & Hexachlorocyclopenta & diene & ND & 330. $\mu g / k g$ \\
\hline Benzyl alcohol & & ND & & 330. $\mu \mathrm{g} / \mathrm{kg}$ & Hexachloroethane & & ND & 330. $\mu \mathrm{g} / \mathrm{kg}$ \\
\hline bis (2-Chloroethy] & 1) ether & ND & & $330 . \mu \mathrm{g} / \mathrm{kg}$ & Indeno $(1,2,3-c, d)$ pyre & & ND & $330 . \mu \mathrm{g} / \mathrm{kg}$ \\
\hline bis (2-Chloroetho) & xy) methane & ND & & $330 . \mu \mathrm{g} / \mathrm{kg}$ & Isophorone & & ND & 330. $\mu \mathrm{g} / \mathrm{kg}$ \\
\hline bis (2-chloroisopr & opyl) ether & ND & & 330. $\mu \mathrm{g} / \mathrm{kg}$ & 2-Methylnaphthalene & & ND & 330. $\mathrm{\mu g} / \mathrm{kg}$ \\
\hline bis (2-Ethylhexyl) & phthalate & ND & & 330. $\mu \mathrm{g} / \mathrm{kg}$ & 2-Methylphenol & & ND & 330. $\mu \mathrm{g} / \mathrm{kg}$ \\
\hline Butylbenzylphthal & & ND & & 330. $\mu \mathrm{g} / \mathrm{kg}$ & 3,4-Methylphenol (iso) & neric pair) & ND & 330. $\mu \mathrm{g} / \mathrm{kg}$ \\
\hline 4-Bromophenyl ph & henyl ether & ND & & 330. $\mu \mathrm{g} / \mathrm{kg}$ & Naphthalene & & ND & 330. $\mu \mathrm{g} / \mathrm{kg}$ \\
\hline Carbazole & & ND & & $330 . \mu \mathrm{g} / \mathrm{kg}$ & 2-Nitroaniline & & ND & 330. $\mu \mathrm{g} / \mathrm{kg}$ \\
\hline 4-Chloroanaline & & $\mathrm{ND}$ & & $330 . \mu \mathrm{g} / \mathrm{kg}$ & 3-Nitroaniline & & ND & 330. $\mu \mathrm{g} / \mathrm{kg}$ \\
\hline 4-Chloro-3-methy & 1 phenol & ND & & 330. $\mu \mathrm{g} / \mathrm{kg}$ & 4-Nitroaniline & & ND & 330. $\mu \mathrm{g} / \mathrm{kg}$ \\
\hline 2-Chloronaphthale & & ND & & 330. $\mu \mathrm{g} / \mathrm{kg}$ & Nitrobenzene & & ND & $330 . \mu \mathrm{g} / \mathrm{kg}$ \\
\hline 2-Chlorophenol & & ND & & 330. $\mu \mathrm{g} / \mathrm{kg}$ & 2-Nitrophenol & & ND & 330. $\mu \mathrm{g} / \mathrm{kg}$ \\
\hline 4-Chlorophenyl pl & henyl ether & ND & & 330. $\mu \mathrm{g} / \mathrm{kg}$ & 4-Nitrophenol & & ND & 330. $\mu \mathrm{g} / \mathrm{kg}$ \\
\hline Chrysene & & ND & & 330. $\mu \mathrm{g} / \mathrm{kg}$ & N-Nitrosodi-n-propyla & mine & ND & 330. $\mu \mathrm{g} / \mathrm{kg}$ \\
\hline Dibenzo $(\mathrm{a}, \mathrm{h})$ anth & hracene & ND & & 330. $\mu \mathrm{g} / \mathrm{kg}$ & N-Nitrosodimethylami & & ND & 330. $\mu \mathrm{g} / \mathrm{kg}$ \\
\hline Dibenzofuran & & ND & & 330. $\mu \mathrm{g} / \mathrm{kg}$ & N-Nitrosodiphenylami & & ND & 330. $\mu \mathrm{g} / \mathrm{kg}$ \\
\hline Di-n-butyl phthala & & ND & & 330. $\mu \mathrm{g} / \mathrm{kg}$ & Pentachlorophenol & & ND & $330 . \mu \mathrm{g} / \mathrm{kg}$ \\
\hline 1,2-Dichlorobenze & ene (o-DCB) & ND & & $330 . \mu \mathrm{g} / \mathrm{kg}$ & Phenol & & ND & $330 . \mu \mathrm{g} / \mathrm{kg}$ \\
\hline 1,3-Dichlorobenze & ene (m-DCB) & ND & & 330. $\mu \mathrm{g} / \mathrm{kg}$ & Phenanthrene & & ND & $330 . \mu \mathrm{g} / \mathrm{kg}$ \\
\hline 1,4-Dichlorobenz & ene ( $p-D C B)$ & ND & & 330. $\mu \mathrm{g} / \mathrm{kg}$ & Pyrene & & ND & 330. $\mu \mathrm{g} / \mathrm{kg}$ \\
\hline 2,4-Dichlorophen & & ND & & 330. $\mu \mathrm{g} / \mathrm{kg}$ & Pyridine & & ND & $330 . \mu \mathrm{g} / \mathrm{kg}$ \\
\hline 3,3'-Dichlorobenz & idine & ND & & 330. $\mu \mathrm{g} / \mathrm{kg}$ & 1,2,4-Trichlorobenzen & & ND & $330 . \mu \mathrm{g} / \mathrm{kg}$ \\
\hline Diethylphthalate & & ND & & 330. $\mu \mathrm{g} / \mathbf{k g}$ & 2,4,5-Trichlorophenol & & ND & $330 . \mu \mathrm{g} / \mathrm{kg}$ \\
\hline 2,4-Dimethylphen & & ND & & $330 . \mu \mathrm{g} / \mathrm{kg}$ & 2,4,6-Trichlorophenol & & ND & 330. $\mu \mathrm{g} / \mathrm{kg}$ \\
\hline
\end{tabular}

QUALITY CONTROL DATA:

\section{Surrogate}

2,4,6-Tribromophenol

2-Fluorobiphenyl

2-Fluorophenol

Nitrobenzene-d5

p-Terphenyl-d14

Phenol-d6

\begin{tabular}{c} 
\% Recovery \\
\hline 58 \\
59 \\
58 \\
60 \\
75 \\
64
\end{tabular}

\section{Acceptable Range}

$19-122$
$30-115$
$25-121$
$23-120$
$18-137$
$24-113$

ND - Not Detected

This report shall not be reproduced except in full, without the written approval of the laboratory. 


\begin{tabular}{|c|c|c|c|c|c|c|c|}
\hline \multirow{2}{*}{$\begin{array}{l}\text { CLIENT: } \\
\text { PROJECT D: } \\
\text { PROJECT \#: } \\
\text { TEST: } \\
\text { METHOD: } \\
\text { MATRIX: }\end{array}$} & \multicolumn{3}{|l|}{$\begin{array}{l}\text { Bechtel Nevada } \\
\text { V1234 } \\
23081\end{array}$} & $\begin{array}{l}\text { CLIENT ID: } \\
\text { DATE SAMPLED: } \\
\text { NEL SAMPLE ID: }\end{array}$ & \multicolumn{3}{|c|}{$\begin{array}{l}\text { Method Blank } \\
\text { NA } \\
\text { 010812-8270-BLK }\end{array}$} \\
\hline & $\begin{array}{l}\text { Semi-Volatile O } \\
\text { EPA } 8270 \\
\text { Solid }\end{array}$ & nic Cor & nds by EPA & $\begin{array}{l}\text { 270C, Dec. } 1996 \\
\text { ANALYST: } \\
\text { EXTRACTED: } \\
\text { ANALYZED: }\end{array}$ & $\begin{array}{l}\mathrm{JRW} \text { - La } \\
8 / 12 / 01 \\
8 / 15 / 01\end{array}$ & as Divisio & \\
\hline \multicolumn{2}{|l|}{ PARAMETER } & $\begin{array}{l}\text { Result } \\
\mu \mathrm{g} / \mathrm{kg}\end{array}$ & $\begin{array}{l}\text { Reporting } \\
\text { Limit }\end{array}$ & PARAMETER & & $\begin{array}{l}\text { Result } \\
\mu \mathrm{g} / \mathrm{kg}\end{array}$ & $\begin{array}{l}\text { Reporting } \\
\text { Limit }\end{array}$ \\
\hline \multicolumn{2}{|l|}{ Acenaphthene } & ND & $330 \mu \mathrm{g} / \mathrm{kg}$ & \multicolumn{2}{|l|}{ Diethylphthalate } & ND & $330 \mu \mathrm{g} / \mathrm{kg}$ \\
\hline \multicolumn{2}{|l|}{ Acenaphthylene } & ND & $330 \mu \mathrm{g} / \mathrm{kg}$ & \multicolumn{2}{|l|}{ 2,4-Dimethylphenol } & ND & $330 \mu \mathrm{g} / \mathrm{kg}$ \\
\hline Aniline & & ND & $330 \mu \mathrm{g} / \mathrm{kg}$ & \multicolumn{2}{|l|}{ Dimethylphthalate } & ND & $330 \mu \mathrm{g} / \mathrm{kg}$ \\
\hline Anthracene & & ND & $330 \mu \mathrm{g} / \mathrm{kg}$ & \multicolumn{2}{|c|}{ 4,6-Dinitro-2-methyl phenol } & ND & $330 \mu \mathrm{g} / \mathrm{kg}$ \\
\hline Azobenzene & & ND & $330 \mu \mathrm{g} / \mathrm{kg}$ & \multicolumn{2}{|c|}{ 2,4-Dinitrotoluene (DNT) } & ND & $330 \mu \mathrm{g} / \mathrm{kg}$ \\
\hline Benzo (a) anthrace & & ND & $330 \mu \mathrm{g} / \mathrm{kg}$ & \multicolumn{2}{|l|}{ 2,6-Dinitrotoluene (DNT) } & ND & $330 \mu g / k g$ \\
\hline Benzo (b) fluorant & hene & ND & $330 \mu \mathrm{g} / \mathrm{kg}$ & \multicolumn{2}{|l|}{ 2,4-Dinitrophenol } & ND & $330 \mu \mathrm{g} / \mathrm{kg}$ \\
\hline Benzo (k) fluorant & hene & ND & $330 \mu \mathrm{g} / \mathrm{kg}$ & \multicolumn{2}{|l|}{ Di-n-octyl phthalate } & ND & $330 \mu \mathrm{g} / \mathrm{kg}$ \\
\hline Benzoic Acid & & ND & $330 \mu \mathrm{g} / \mathrm{kg}$ & \multicolumn{2}{|l|}{ Fluoranthene } & ND & $330 \mu \mathrm{g} / \mathrm{kg}$ \\
\hline Benzo $(g, h, i)$ peryl & & ND & $330 \mu \mathrm{g} / \mathrm{kg}$ & \multicolumn{2}{|l|}{ Fluorene } & ND & $330 \mu \mathrm{g} / \mathrm{kg}$ \\
\hline Benzo (a) pyrene & & ND & $330 \mu \mathrm{g} / \mathrm{kg}$ & \multicolumn{2}{|l|}{ Hexachlorobenzene } & ND & $330 \mu \mathrm{g} / \mathrm{kg}$ \\
\hline Benzyl alcohol & & ND & $330 \mu \mathrm{g} / \mathrm{kg}$ & \multicolumn{2}{|l|}{ Hexachlorobutadiene } & ND & $330 \mu \mathrm{g} / \mathrm{kg}$ \\
\hline bis (2-Chloroethyl & ether & ND & $330 \mu \mathrm{g} / \mathrm{kg}$ & \multicolumn{2}{|c|}{ Hexachlorocyclopentadiene } & ND & $330 \mu \mathrm{g} / \mathrm{kg}$ \\
\hline bis (2-Chloroethox & y) methane & ND & $330 \mu \mathrm{g} / \mathrm{kg}$ & \multicolumn{2}{|c|}{ Hexachloroethane } & ND & $330 \mu \mathrm{g} / \mathrm{kg}$ \\
\hline bis (2-chloroisopro & pyl) ether & ND & $330 \mu \mathrm{g} / \mathrm{kg}$ & \multicolumn{2}{|l|}{ Indeno $(1,2,3-c, d)$ pyrene } & ND & $330 \mu \mathrm{g} / \mathrm{kg}$ \\
\hline bis (2-Ethylhexyl) & Shthalate & ND & $330 \mu g / k g$ & \multicolumn{2}{|l|}{ Isophorone } & ND & $330 \mu \mathrm{g} / \mathrm{kg}$ \\
\hline Butylbenzylphthal & & ND & $330 \mu \mathrm{g} / \mathrm{kg}$ & \multicolumn{2}{|l|}{ 2-Methylnaphthalene } & ND & $330 \mu \mathrm{g} / \mathrm{kg}$ \\
\hline 4-Bromophenyl ph & enyl ether & ND & $330 \mu \mathrm{g} / \mathrm{kg}$ & \multicolumn{2}{|l|}{ 2-Methylphenol } & ND & $330 \mu \mathrm{g} / \mathrm{kg}$ \\
\hline Carbazole & & ND & $330 \mu \mathrm{g} / \mathrm{kg}$ & \multicolumn{2}{|c|}{ 3,4-Methylphenol (isomeric pair) } & ND & $330 \mu \mathrm{g} / \mathrm{kg}$ \\
\hline 4-Chloroanaline & & ND & $330 \mu \mathrm{g} / \mathrm{kg}$ & \multicolumn{2}{|c|}{ Naphthalene } & ND & $330 \mu \mathrm{g} / \mathrm{kg}$ \\
\hline 4-Chloro-3-methyl & phenol & ND & $330 \mu g / k g$ & 2-Nitroaniline & & ND & $330 \mu \mathrm{g} / \mathrm{kg}$ \\
\hline 2-Chloronaphthale & & ND & $330 \mu \mathrm{g} / \mathrm{kg}$ & 3-Nitroaniline & & ND & $330 \mu \mathrm{g} / \mathrm{kg}$ \\
\hline 2-Chlorophenol & & ND & $330 \mu \mathrm{g} / \mathrm{kg}$ & 4-Nitroaniline & & ND & $330 \mu \mathrm{g} / \mathrm{kg}$ \\
\hline 4-Chlorophenyl ph & enyl ether & ND & $330 \mu \mathrm{g} / \mathrm{kg}$ & Nitrobenzene & & ND & $330 \mu \mathrm{g} / \mathrm{kg}$ \\
\hline Chrysene & & ND & $330 \mu \mathrm{g} / \mathrm{kg}$ & 2-Nitrophenol & & ND & $330 \mu \mathrm{g} / \mathrm{kg}$ \\
\hline Dibenzo $(2, h)$ anth & racene & ND & $330 \mu \mathrm{g} / \mathrm{kg}$ & 4-Nitrophenol & & ND & $330 \mu g / k g$ \\
\hline Dibenzofuran & & ND & $330 \mu \mathrm{g} / \mathrm{kg}$ & N-Nitrosodi-n-propylan & mine & ND & $330 \mu g / k g$ \\
\hline Di-n-butyl phthala & & ND & $330 \mu \mathrm{g} / \mathrm{kg}$ & N-Nitrosodimethylamin & & ND & $330 \mu g / k g$ \\
\hline 1,2-Dichlorobenze & ne (o-DCB) & ND & $330 \mu \mathrm{g} / \mathrm{kg}$ & N-Nitrosodiphenylamin & & ND & $330 \mu \mathrm{g} / \mathrm{kg}$ \\
\hline 1,3-Dichlorobenze & $n e(m-D C B)$ & ND & $330 \mu g / k g$ & Pentachlorophenol & & ND & $330 \mu \mathrm{g} / \mathrm{kg}$ \\
\hline 1,4-Dichlorobenze & $n e(p-D C B)$ & ND & $330 \mu \mathrm{g} / \mathrm{kg}$ & Phenol & & ND & $330 \mu \mathrm{g} / \mathrm{kg}$ \\
\hline 2,4-Dichloropheno & & ND & $330 \mu \mathrm{g} / \mathrm{kg}$ & Phenanthrene & & ND & $330 \mu \mathrm{g} / \mathrm{kg}$ \\
\hline 3,3'-Dichlorobenzi & dine & ND & $330 \mu g / k g$ & Pyrene & & ND & $330 \mu \mathrm{g} / \mathrm{kg}$ \\
\hline
\end{tabular}

\section{QUALITY CONTROL DATA:}

$\begin{array}{ll}\text { - Surrogate } & \\ \text { 2,4,6-Tribromophenol } \\ \text { 2-Fluorobiphenyl } \\ \text { 2-Fluorophenol } \\ \text { Nitrobenzene-d5 } \\ \text { p-Terphenyl-d14 } \\ \text { Phenol-d6 }\end{array}$

\begin{tabular}{c} 
\% Recovery \\
\hline 71 \\
57 \\
55 \\
51 \\
84 \\
56
\end{tabular}

\begin{tabular}{l} 
Acceptable Range \\
\hline $19-122$ \\
$30-115$ \\
$25-121$ \\
$23-120$ \\
$18-137$ \\
$24-113$
\end{tabular}

ND - Not Detected

This report shall not be reproduced except in full, without the written approval of the laboratory. 


\begin{tabular}{|c|c|c|c|c|c|c|c|c|}
\hline $\begin{array}{l}\text { CLIENT: } \\
\text { PROJECT ID: } \\
\text { PROJECT \#: }\end{array}$ & $\begin{array}{l}\text { Bechtel Nevada } \\
\text { V1234 } \\
23081\end{array}$ & & & & & & & \\
\hline $\begin{array}{l}\text { TEST: } \\
\text { METHOD: } \\
\text { ORDER ID: }\end{array}$ & $\begin{array}{l}\text { Total Extractable } \\
\text { EPA 8015M } \\
\text { L0108079 }\end{array}$ & Petroleum Hyd & Hocarbons 1 & & Method $8015 \mathrm{M}$ & , December & 1996 & \\
\hline MATRIX: & Solid & & & & & JALYST: & PXC - Division & \\
\hline $\begin{array}{l}\text { CLIENT } \\
\text { SAMPLE ID } \\
\end{array}$ & $\begin{array}{c}\text { SAMPLE } \\
\text { DATE } \\
\end{array}$ & $\begin{array}{c}\text { NEL } \\
\text { SAMPLE ID } \\
\end{array}$ & $\begin{array}{l}\text { RESULT } \\
\text { mg/kg }\end{array}$ & C.R. & $\begin{array}{c}\text { Reporting } \\
\text { Limit }\end{array}$ & $\begin{array}{l}\text { Surrogate } \\
\text { Recovery }\end{array}$ & EXTRACTED & ANALYZE \\
\hline 079903-0-A & $8 / 8 / 01$ & L0108079-01 & ND & $\mathrm{ND}$ & 20. $\mathrm{mg} / \mathrm{k}$ & 75 & $8 / 10 / 01$ & $8 / 10 / 01$ \\
\hline 079903-0-B & $8 / 8 / 01$ & L0108079-02 & ND & ND & $20 . \mathrm{mg} / \mathrm{k}$ & 69 & $8 / 10 / 01$ & $8 / 10 / 01$ \\
\hline 079903-0-C & $8 / 8 / 01$ & L0108079-03 & ND & ND & 20. $\mathrm{mg} / \mathrm{k}$ & 74 & $8 / 10 / 01$ & $8 / 10 / 01$ \\
\hline
\end{tabular}

\section{C.R.: Carbon Range} QUALITY CONTROL DATA (Total for Diesel Range):

\section{Sample ID}

Blank, 010810TP -BLK

LCS, 010810TPHS-1-LCS

LCSD, 010810TPHS-1-LCSD

MS, 010810TPHS-1-MS

MSD, 010810TPHS-1-MSD

* Surrogate used was Octacosane, acceptance limits 55-130\%.

Result Acceptable Range Surrogate Recovery* Sample Number

ND $<$

< $\quad 20 \mathrm{mg} / \mathrm{kg}$

$84 \%$

$66 \%$

$59 \%$

$78 \%$

$71 \%$
NA

NA

NA

L0108079-01

L0108079-01

ND - Not Detected

This report shall not be reproduced except in full, without the written approval of the laboratory. 


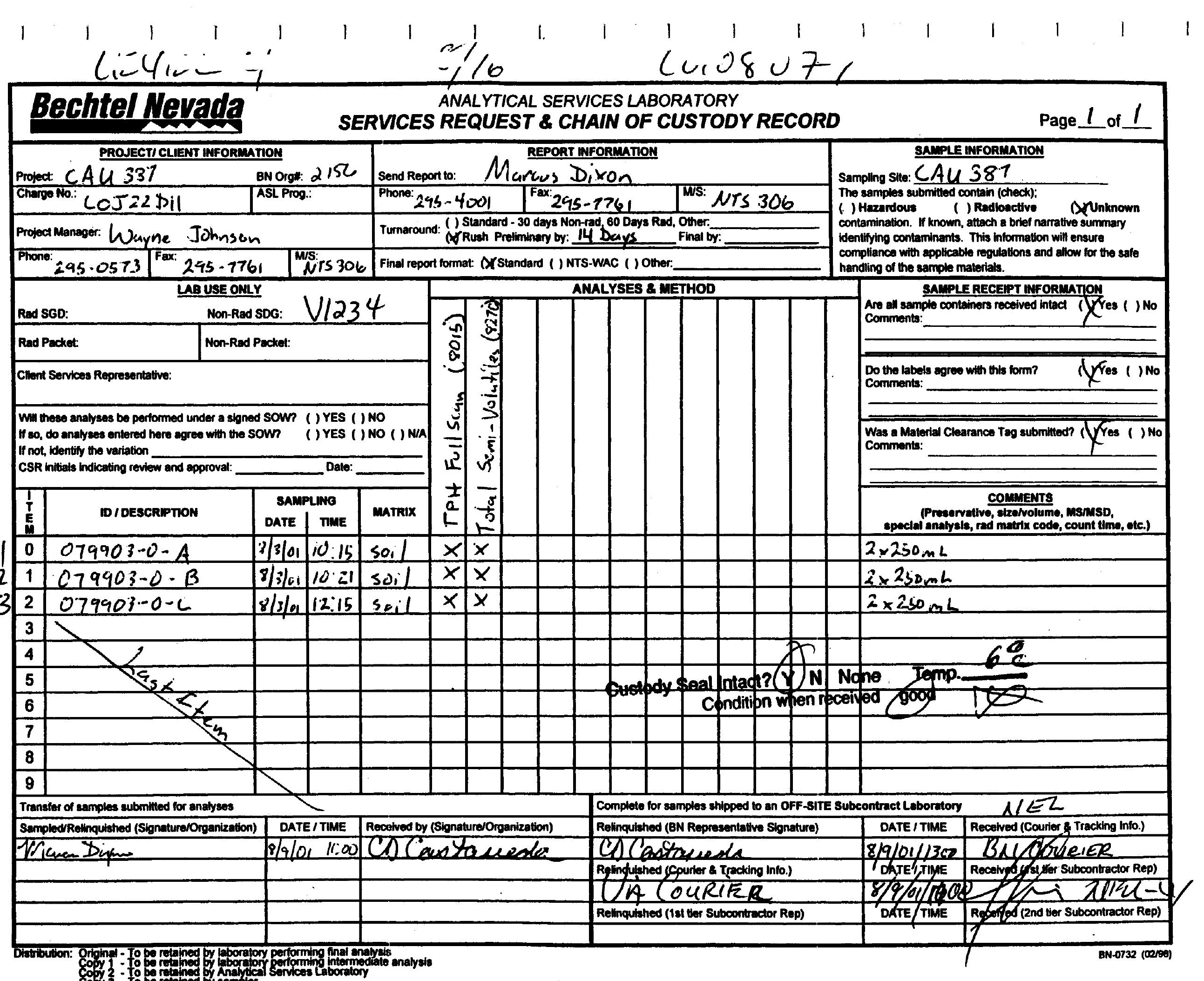




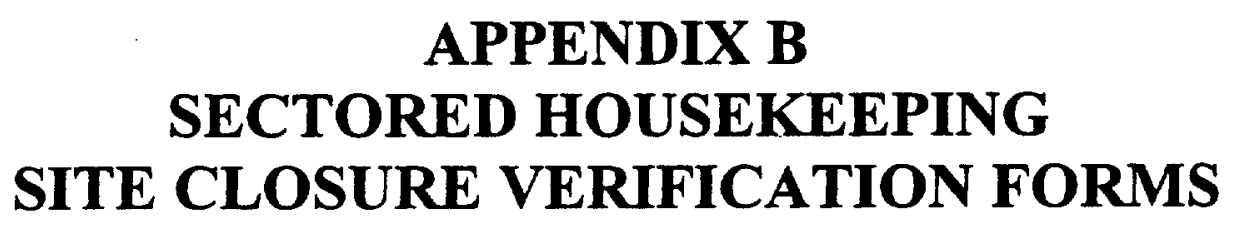




\section{Sectored Housekeeping Site Closure Verification Form}

Closure Verification Date: 08/17/2001

CAS Number (if applicable): 02-99-02

CAU Number (if applicable): 387

Sector Desingnation: NTS

Housekeeping Site General Location: Nevada Test Site, U-2er crater

Elevation: 1,297 meters (m)

Northing: 4,112,976.24 m (UTM Zone: 11) Latitude: 3709.6782

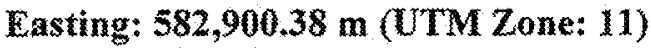

Longitude: 11604.0328

Coordinate/Elevation Data Obtained from: North American Datum, 1927.

Site Access Route: Proceed north on the Mercury Highway to Rainier Mesa Road. Tum left (northwest) onto Rainier Mesa Road and proced to 2-07 Road. Turn right onto the 2.07 Road (east) and contmue for 2 miles to RSM 2L-20. The U-2er crater will be due south, across the road from RAD Safe Marker (RSM) $2 L-20$.

\begin{tabular}{|c|l|}
\hline Waste Item(s) Originally at Site & \multicolumn{1}{|c|}{ Apparent Waste Type $^{*}$} \\
\hline Epoxy tar, wood, metal, and rope & Ordinary \\
\hline
\end{tabular}

* Ordinary, Sera Metal, Asbestos, PCB, Solvagedole, Hatardous, Radioscrive. Mixed, Unknown, Other

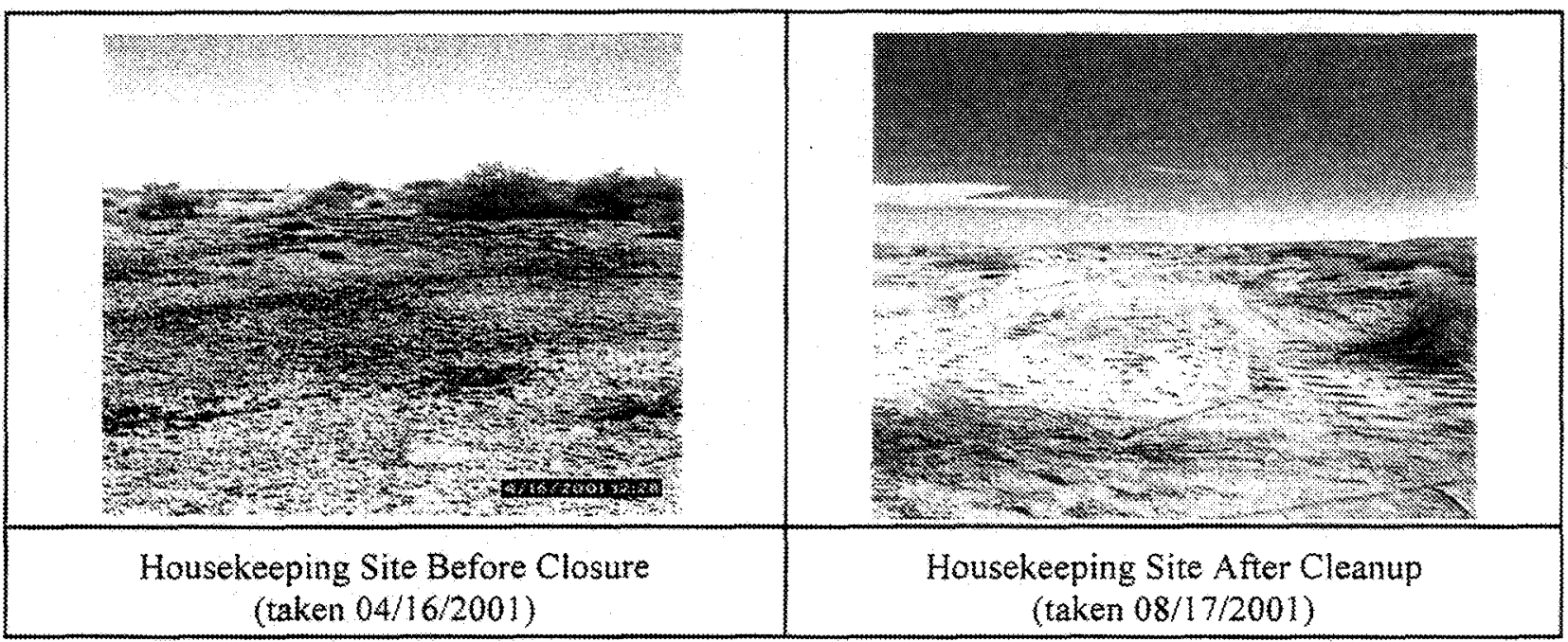

Current Site Description/Observations: This Corrective Action Site (CAS) was first identified by an International Technology Corporation (TT) field crew in a site survey performed on October $20,1993$. The epoxy tar and debris were removed on August 17,200l, using shovels and a front-end loader. The material was placed into B-25 containers due to its potential to be low-level waste (LLW). The site is currently clear of all waste material.

\section{$\mathrm{X}$ No Further Action Required at Housekeeping Site}

Marcus Dixon

Pos Disio

Corrective Action Coordinator/Designee

Signature

$10 / 25 / 20 \%$

Date 


\title{
Sectored Housekeeping Site Closure Verification Form
}

\author{
Closure Verification Date: 08/20/2001 \\ CAS Number (if applicable): 02-99-03 (east spill) \\ CAU Number (if applicable): 387
}

Sector Designation: NTS

Housekeeping Site General Location: Nevada Test Site, U-2 2 crater

Elevation: 1,398 meters $(m)$

Northing: $4,109,71.61 \mathrm{~m}$ (UTM Zone: 11$)$

Easting: 580,914.36 m (UTM Zone: 11)

Latitude: 3707.6311

Longitude: 11605.3994

\section{Coordinate/Elevation Data Obtained from: North American Datum, 1927.}

Site Access Route: Proceed on the Mercury Highway north to Rainier Mesa Road. Turn left (northwest) onto Rainier Mesa Road and proceed 300 feet past 2-03 Road. Tum left (west) onto the dirt road at a sign that indicates "U-2fg". Continue west on the dirt road for 0.85 mile just before a yellow optical survey box. Bear left (south) down the dir road (toward soil piles) for another 0.3 mile to the U-2fd crater. Spills are located northwest of the U-2fd crater and southwest of two large dir mounds.

\begin{tabular}{|c|l|}
\hline Waste Item(s) Originally at Site & \multicolumn{1}{|c|}{ Apparent Waste Type* } \\
\hline Epoxy tar & Ordinary \\
\hline
\end{tabular}

* Ordinary, Scrap Metal, Asbestos, PCB, Salvageable, Hazardous, Radioactive, Mixed, Unknown, Orier

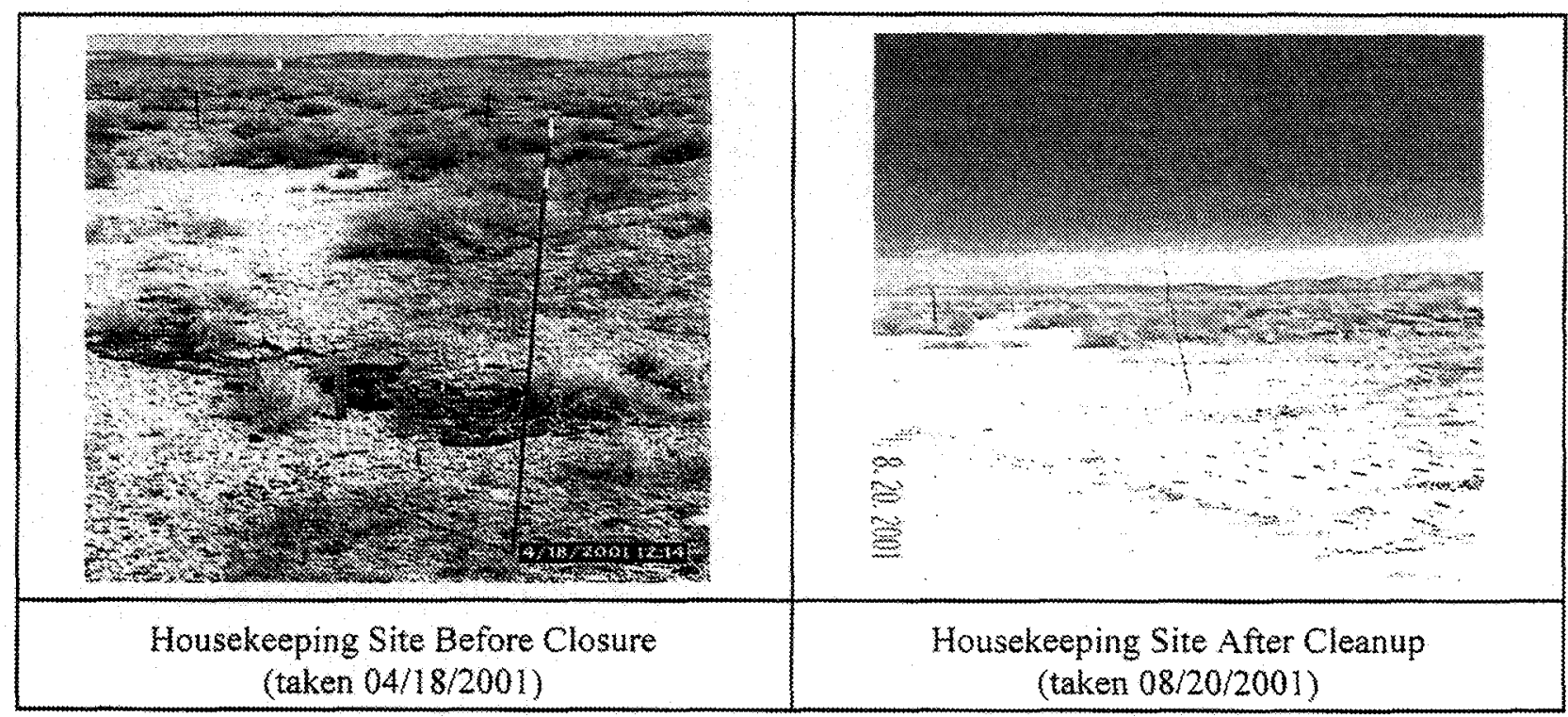

Current Site Description/Observations: This Corrective Action Site (CAS) was frst identified by an International Technology Corporation (IT) ficld crew in a site survey performed on October $22,1993$. The epoxy tar was removed on August 20, 2001, using shovels and a front-end loader and transported to the Area 6 Hydrocarbon Landfill. The site is currently clear of all waste material.

$\mathrm{x}$ No Further Action Required at Housekeeping Sitte

$\begin{array}{lcc}\text { Marcus Dixon } & 1 / 2 & 10 / 25 / 200 ; \\ \text { Corrective Action Coordinator/Designee } & \text { Signature } & \text { Date }\end{array}$




\section{Sectored Housekeeping Site Closure Verification Form}

Closure Verification Date: 08/20/2001

CAS Number (if applicable): 02-99-03 (west spill)

CAU Number (if applicable): 387

Sector Designation: NTS

Housekeeping Site General Location: Nevada Test Site, U-2fd crater

Elevation: 1,398 meters $(\mathrm{m})$

Northing: 4,109,161.52 m (UTM Zone: 11) Lafitude: 3707.6258

Easting: $580,884.85 \mathrm{~m}$ (UTM Zone: 11 )

Longitude: 11605.4194

Coordinaterevation Data Obtained from: North American Datum, 1927.

Site Access Route: Proceed on the Mercury Highway north to Ramier Mesa Road. Turn left (northwest) onto Rainier Mesa Road and proceed 300 feet past 2.03 Road. Turn left (west) onto the dirt road at a sign that indicates "U $-2 \mathrm{fg}^{\text {". }}$. Contimue west on the dirt road for 0.85 mile just before a yellow optical survey box. Bear left (soutin) down the dirt road (toward soil piles) for another 0.3 mile to the U-2fd crater. Spills are located northwest of the U- $2 \mathrm{fd}$ crater and southwest of two large dirt mounds.

\begin{tabular}{|c|l|}
\hline Waste Item(s) Originally at Site & \multicolumn{1}{c|}{ Apparent Waste Type* } \\
\hline Epoxy atr & Ordinary \\
\hline
\end{tabular}

* Ordirary, Scrap Metal, Askestos, PCB, Salvageable Hazardous, Radioactive, Mixed, Unknown, Other

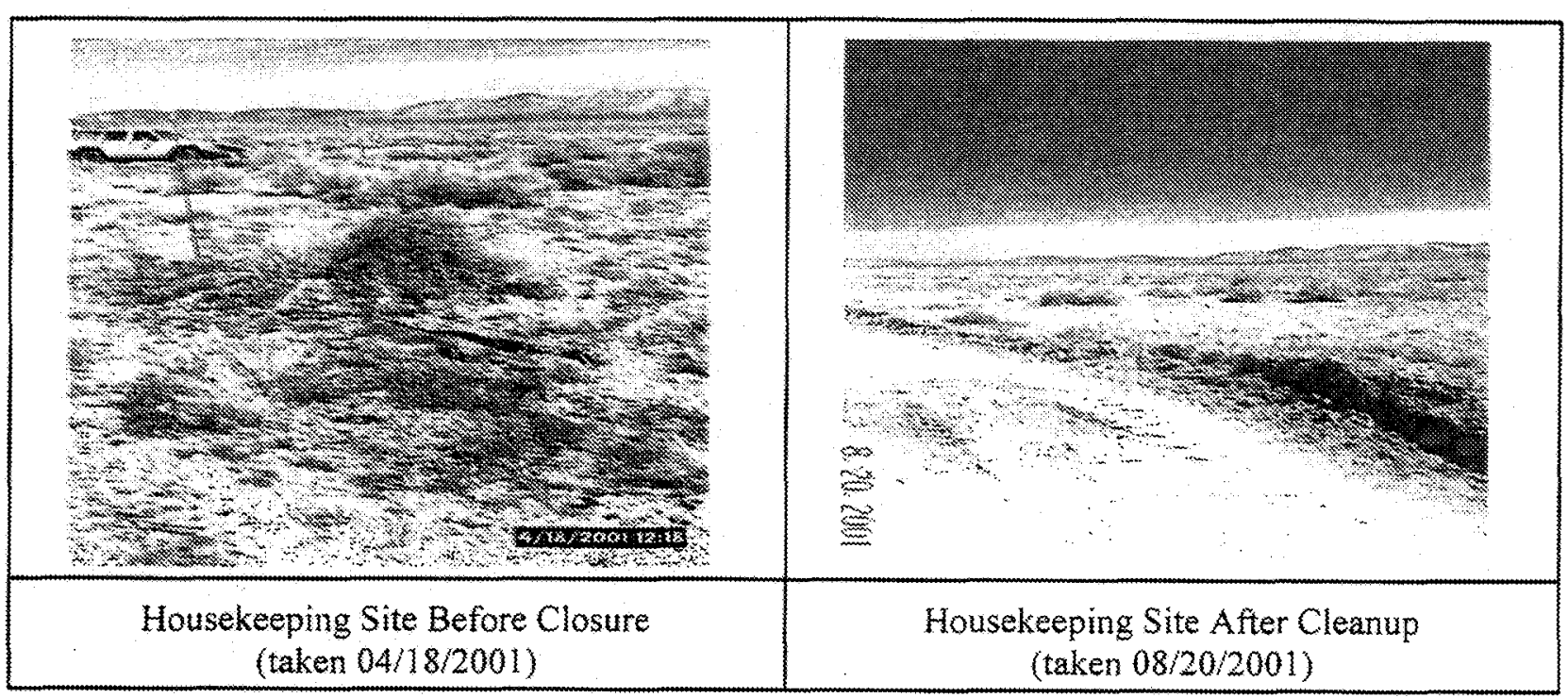

Current Site Description/Observations: This Corrective Action Site (CAS) was first identified by an International Technology Corporation (IT) field crew in a site survey performed on October $22,1993$. The epoxy tar was removed on August 20,2001, using shovels and a front-end loader, and transported to the Area 6 Hydrocarbon Landfill. The site is currently clear of all waste material.

$\times$ No Furthex Action Required at Housekeeping Site

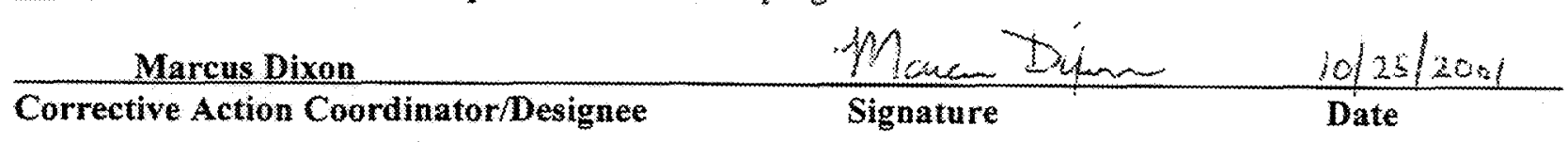




\section{Sectored Housekeeping Site Closure Verification Form}

Closure Verification Date: $08 / 20 / 2001$

CAS Number (if applicable): 02-99-04

CAU Number (if applicable): 387

Sector Designation: NTS

Housekeeping Site General Location: Nevada Test Site, U-2bq crater

Elevation: 1,314 meters (m)

Northing: 4,112,594.16 m (UTM Zone: 11)

Latitude: 3709.4788

Easting: $581,529.64 \mathrm{~m}$ (UTM Zone: 11 )

Longitude: 11604.9615

\section{Coordinate/Elevation Data Obtained from: North American Datum, 1927.}

Site Access Route: Proceed on the Mercury Highway north to Rainier Mesa Road. Turn left (northwest) onto Rainier Mesa Road to Area 2 Camp. Turn right (east) onto the 2.07 Road and proceed for approximately 1.8 miles. Turn right (south) onto a dirt road and proceed for approximately 0.3 mile. Turn left (east) onto another dirt road to the U-2bq crater.

\begin{tabular}{|c|c|}
\hline Waste Item(s) Originally at Site & Apparent Waste Type* \\
\hline Concrete/cement, metal fence post, and wood & Industrial solid waste and ordinary \\
\hline
\end{tabular}

* Ordinany, Scrap Metal, Asbestos, PCB. Salvageable, hazardous, Radionctive, Mixed, Uniknown, Other

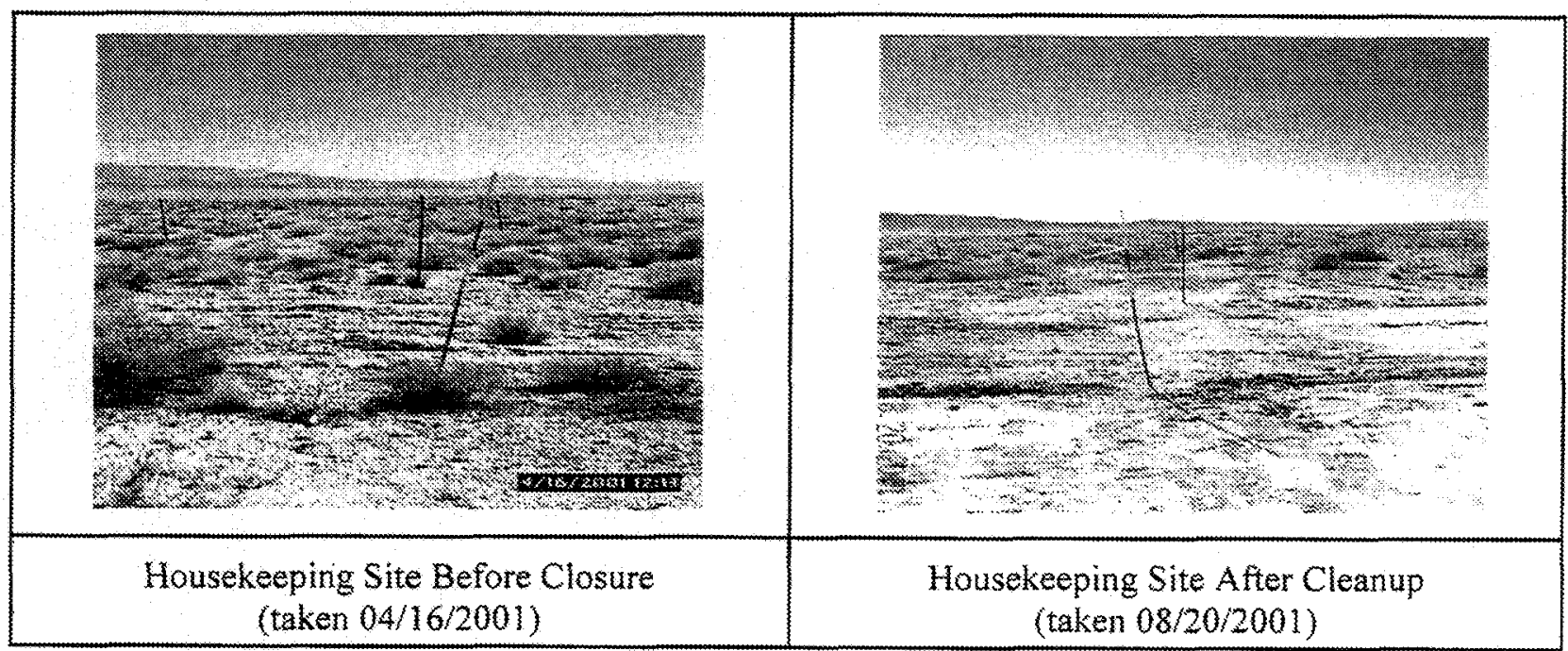

Current Site Description/Observations: This Corrective Action Site (CAS) was first identified by an International Technology Corporation (IT) field crew in a site survey performed on October $20,1993$. The concrete/cement and debris were removed on August 20,2001, using a front-end loader and transported to the Area 9 U10 Landfill. The site is currently clear of all waste material.

$\mathrm{X}$ No Further Action Required at Housekeeping Site

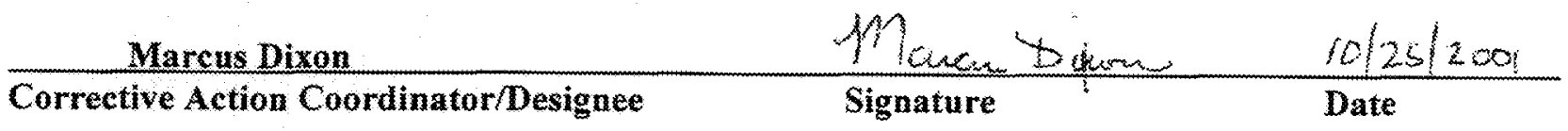




\section{Sectored Housekeeping Site Closure Verification Form}

Closure Verification Date: 08/10/2001

CAS Number (if applicable): 02-99-11

CAU Number (if applicable): 387

Sector Designation: NTS

Housekeeping Site General Location: Nevada Test Site, Power Line Road

Elevation: 1,305 meters (m)

Northing: 4,113,251.49 m (UTM Zone: 11) Latitude: 3709.8367

Easting: 581,064.95 m (UTM Zone: 11) Longitude: 11605.2712

Coordinate/Elevation Data Obtained from: North American Datum, 1927.

Site Access Route: Proceed on Mercury Highway north to Rainier Mesa Road, turn left (northwest) to the 2.07 Road. Turn right (east) onto 2.07 Road and proceed approximately 0.85 mile to a power line road on the south side of 2.07 Road. Tum right (south) onto the power Yine road and proceed approximately 0.1 mile to the former location of the substation where a coarse gravel bed remains on the left (west) side of the road. The spill is on the east side of the road in the wash.

\begin{tabular}{|l|l|}
\hline Waste Item(s) Originally at Site & \multicolumn{1}{|c|}{ Apparent Waste Type* $^{\text {Apte }}$} \\
\hline Epoxy & Ordinary \\
\hline
\end{tabular}

* Ordinary, Scrap Metal. Asbestos, PCB, Salvageable, Gazardous, Radioactive, Mixed. Unknown, Other

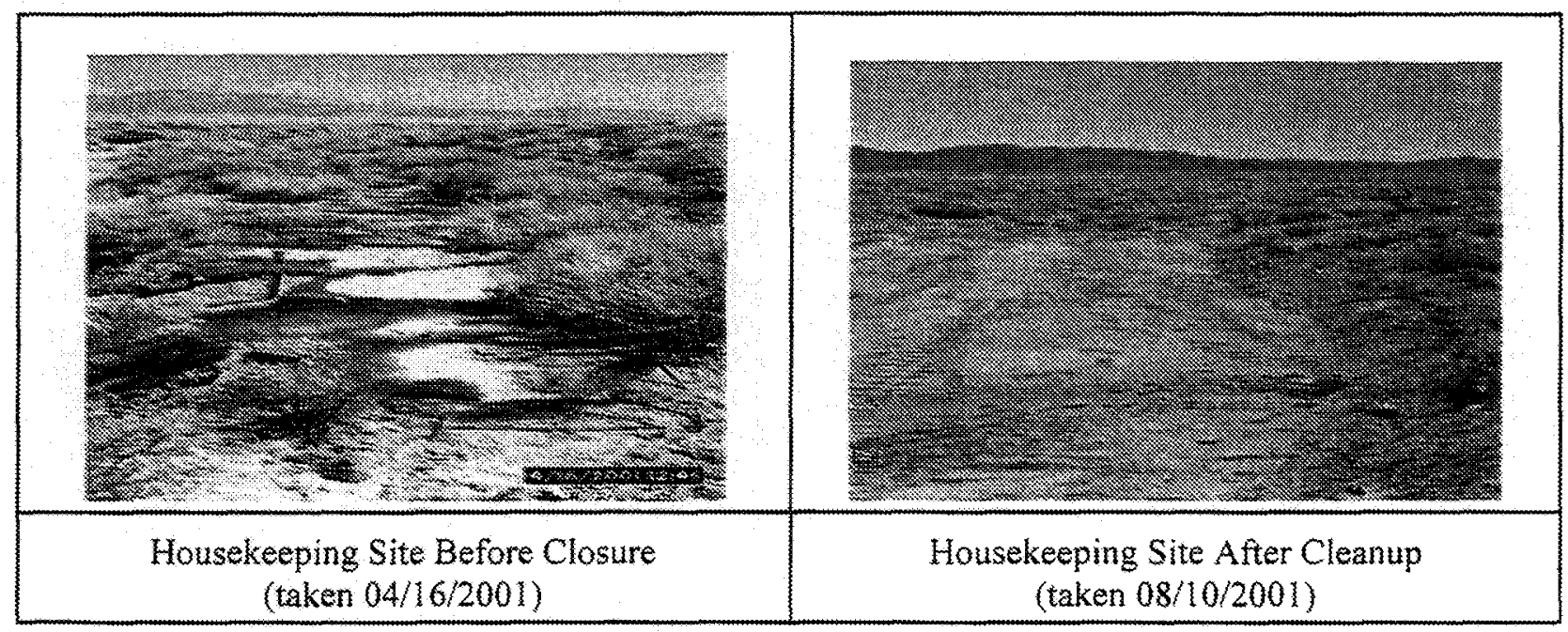

Current Site Description/Observations: This Corrective Action Site (CAS) was first identified by an International Technology Corporation (IT) field crew in a site survey performed on May 10, 1994. The epoxy was removed on August 10,2001, using shovels and a front-end loader. The material was transported to the Area $6 \mathrm{Hydrocarbon} \mathrm{Landfill.} \mathrm{The} \mathrm{site} \mathrm{is} \mathrm{currently} \mathrm{clear} \mathrm{of} \mathrm{all} \mathrm{waste} \mathrm{material.}$

$X$ No Further Action Required at Housekeeping Site

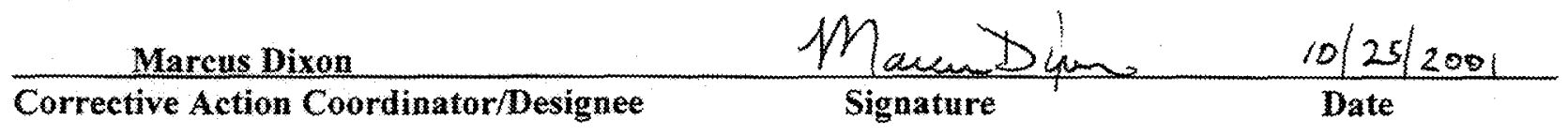




\section{Sectored Housekeeping Site Closure Verification Form}

Closure Verification Date: 08/20/2001

CAS Number (if applicable): 03-22-22

CAU Number (if applicable): 387

Sector Designation: NTS

Housekeeping Site General Location: Nevada Test Site, U-3ev-1 shaft

Elevation: 1,224 meters (m)

Northing: 4,101,090.03 m (UTM Zone: 11)

Latitude: 3703.2109

Easting: $590,011.20 \mathrm{~m}$ (UTM Zone: 11)

Longitude: 11559.3137

Coordinate/Elevation Data Obtained from: North American Datum, 1927.

Site Access Route: Proceed on Mercury Highway north to 3-03 Road. Tum right (east) on 3-03 Road and proceed for 3.5 miles to U-3ev-l shaft on the nortl side of the road. The spill site is located on the east side of the fence near the northeast comer of the fenced area.

\begin{tabular}{|c|l|}
\hline \multicolumn{1}{|c|}{ Waste Item(s) Originally at Site } & \multicolumn{1}{c|}{ Apparent Waste Type* $^{*}$} \\
\hline $\begin{array}{l}\text { Stained/dark soil, steel cables, drum lids, } \\
\text { electrical box, electrical cables, bolts, and wood }\end{array}$ & Ordinary \\
\hline
\end{tabular}

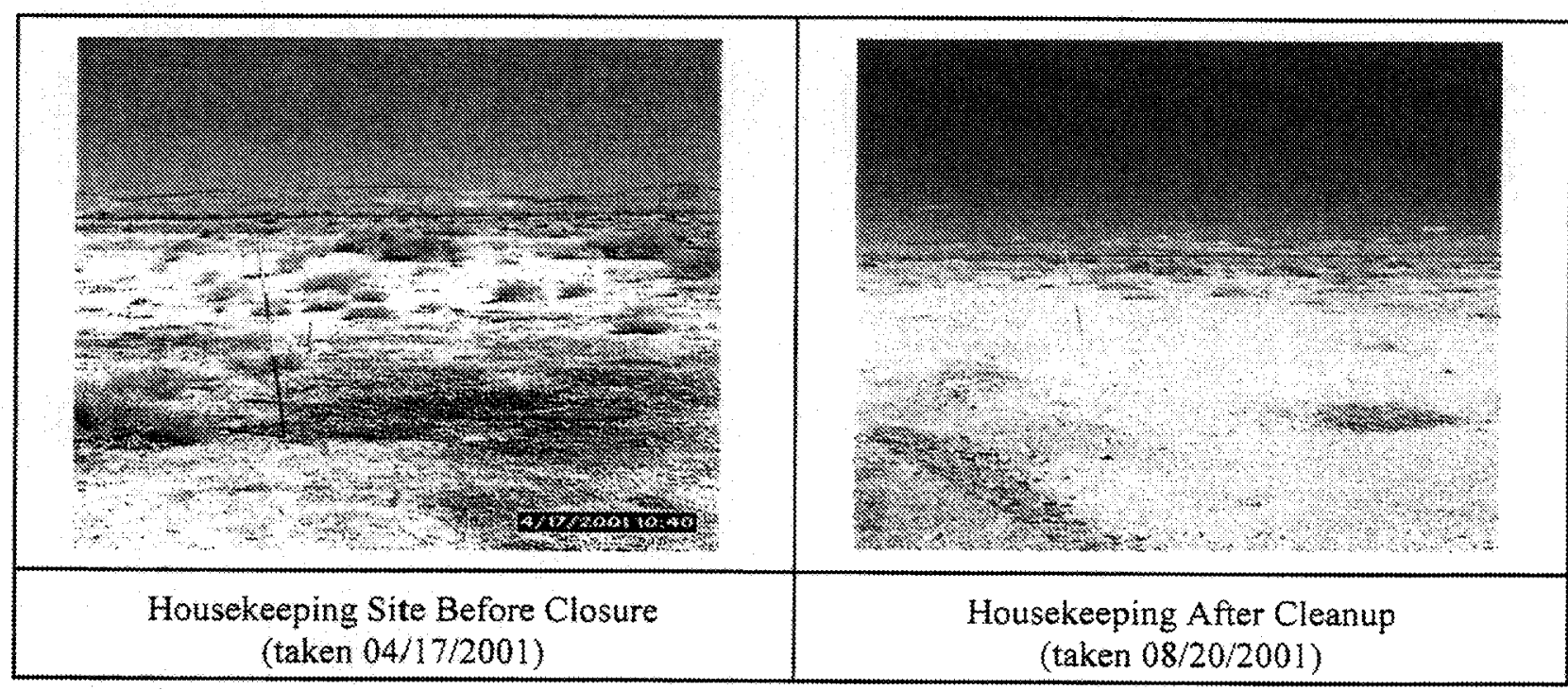

Current Site Description/Observations: This Corrective Action Site (CAS) was first identified by an International Technology Corporation (IT) field crew in a site survey persormed on August 24, 1993. The stained soil and debris were removed on August 20,2001, using a front-end loader and transported to the Area 9 U10c Landfill. The site is currently clear of all waste material.

$\mathrm{X}$ No Further Action Requirred at Housekeeping Site

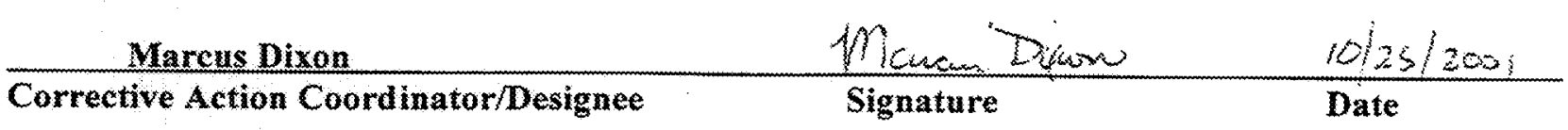




\section{Sectored Housekeeping Site Closure Verification Form}

Closure Verification Date: $08 / 20 / 2001$

CAS Number (if applicable): 03-44-03 (Sites "A" and "B")

CAU Number (if applicable): 387

Sector Designation: NTS

Housekeeping Site General Location: Nevada Test Site, U-3mk crater

Elevation: 1,315 meters $(\mathrm{m})$

Northing: 4,102,639.93 m (UTM Zone: 11) Latitude: 3704.0521

Easting: $\$ 89,482.58 \mathrm{~m}$ (UTM Zone: 11 )

Longitude: 11559.6593

Coordinate/Elevation Data Obtained from: North American Datim, $192 \%$.

Site Access Route: Proceed on Mercury Highway north to 3-03 Road. Turn right (east) on 3403 Road and proceed to 3-05 Road. Turn left (north) on 3-05 Road and proceed approximately 1.4 miles to E-Road. Turn right (east) onto E-Road and proceed approximately 1.5 miles to a three-way fork in the road. The site is located at the northwest side of the crater (look to the right).

\begin{tabular}{|l|l|}
\hline Waste Item(s) Originally at Site & \multicolumn{1}{|c|}{ Apparent Waste Type* } \\
\hline Stained soil & Ordinary \\
\hline Ordinary, Serap Metal Asbestos, $P C B$, Salqageable, Hazarbous, Radiodctive, Mixed, Unknown, Other
\end{tabular}

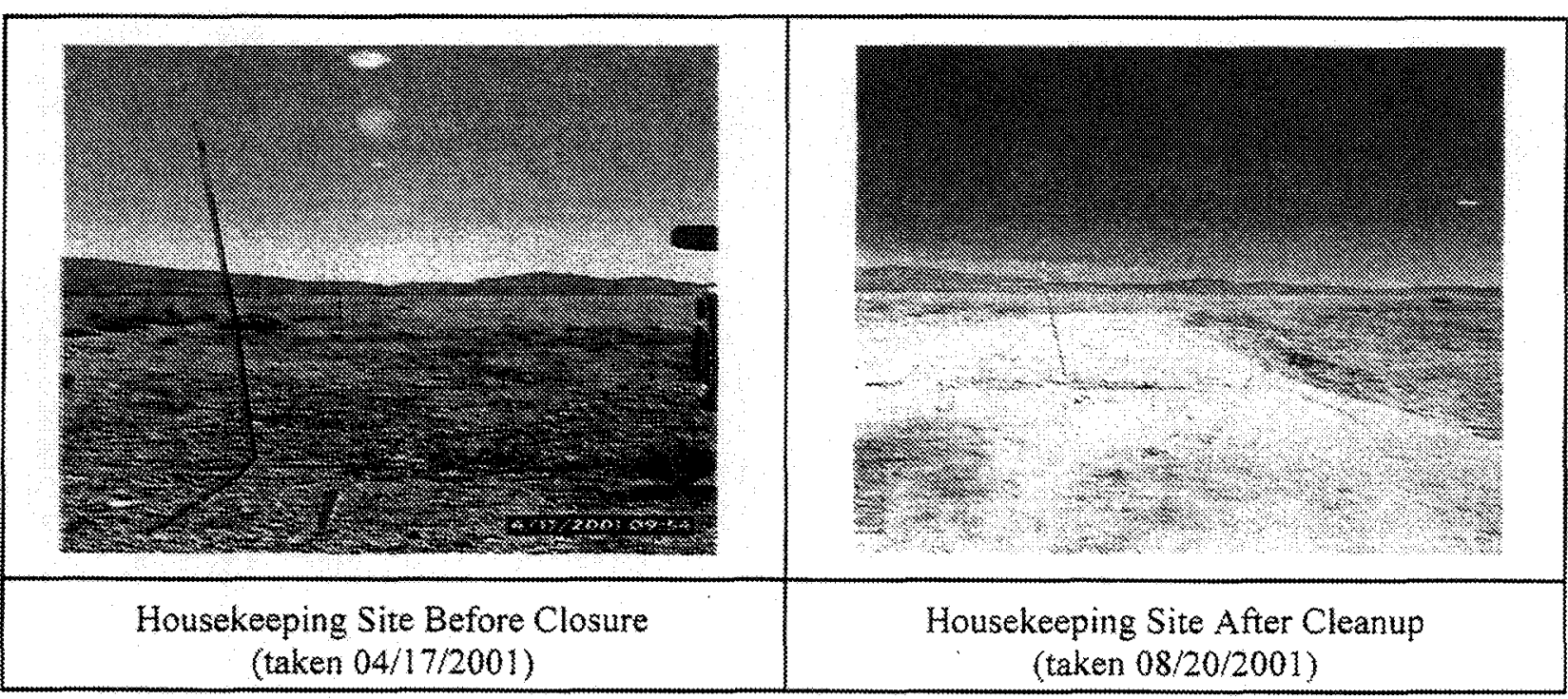

Current Site Description/Observations: This Corrective Action Site (CAS) was first identified by an International Technology Corporation (T) field crew in site survey performed on August 11, 1993. The stained soil was removed on August 20,2001, using a front-end loader and transported to the Area 6 Hydrocarbon Landfill. The site is currently clear of all waste material.

$\mathrm{X}$ No Further Action Required at Housekeeping Site

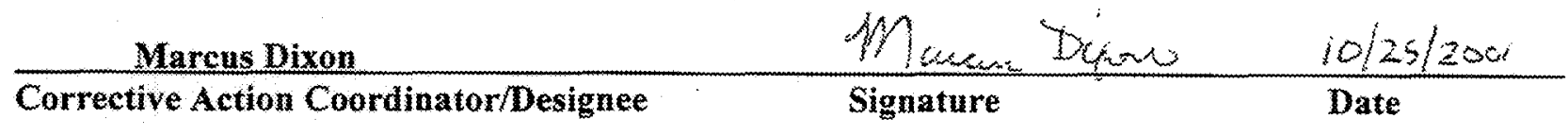




\section{Sectored Housekeeping Site Closure Verification Form}

Closure Verification Date: 07/25/2001

CAS Number (if applicable): 03-99-01

CAU Number (if applicable): 387

Sector Designation: NTS

Housekeeping Sìte General Location: Nevada Test Site, U-3gn crater

Elevation: 1,156 meters $(\mathrm{m})$

Northing: 4,097,559.96 m (UTM Zone: 11) Latitude: 3701.3271

Easting: $585,506.48 \mathrm{~m}$ (UTM Zone: 11 )

Longitude: 11602.3772

Coordinate/Elevation Data Obtained from: North American Datum, 1927.

Site Access Route: Proceed on Mercury Highway north to Angle Road. Tum right (northeast) on Angle Road and proced 0.85 mile. The spill is approximately 0.1 mile of the road (east) near the U-3gn crater.

\begin{tabular}{|c|l|}
\hline Waste Item(s) Originally at Site & \multicolumn{1}{|c|}{ Apparent Waste Type $^{*}$} \\
\hline Epoxy tar, metal, wood debris, and wire & Ordinary \\
\hline
\end{tabular}

* Ordinary, Serap Metal, Asbestos, PCB, Salvageable, Hazardous, Radicactive. Mixed. Unkrown, Other

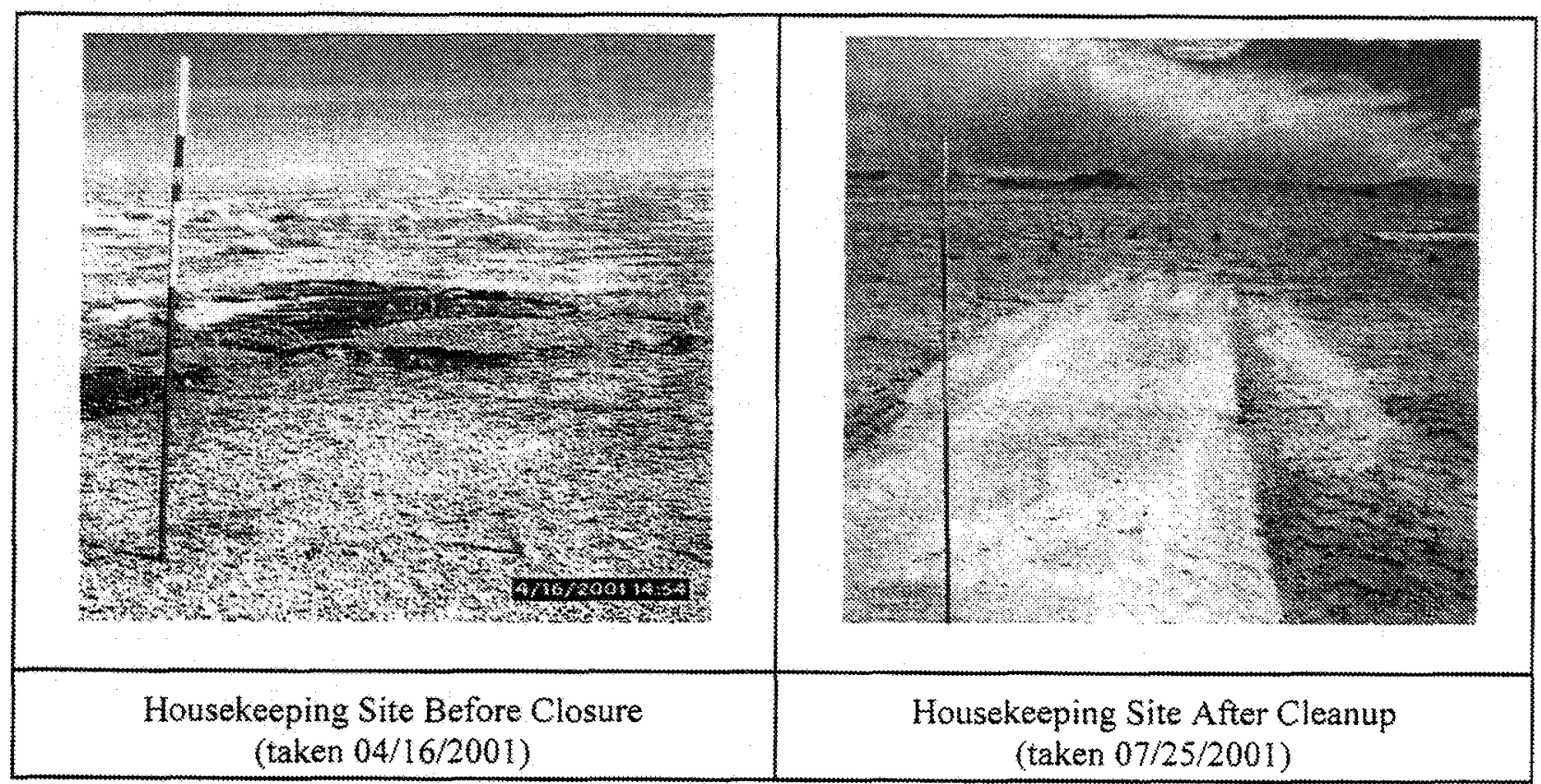

Current Site Description/Observations: This Corrective Action Site (CAS) was first identified by an International Technology Corporation (IT) field crew in a site survey perfomed on October 5, 1993. The epoxy tar and debris were removed on July 25,2001 , using shovels and a front-end loader and transported to the Area $6 \mathrm{Hydrocarbon}$ Landfill. The site is currently clear of all waste material.

\section{$\times$ No Further Action Required at Housekeeping Site}

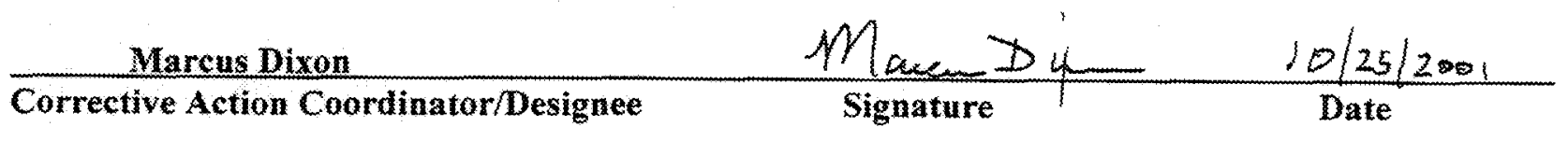




\section{Sectored Housekeeping Site Closure Verification Form}

Closure Verification Date: 07/24/2001

CAS Number (if applicable): 03-99-03

CAU Number (if applicable): 387

Sector Designation: NTS

Housekeeping Site General Location: Nevada Test Site, U-3gn crater

Elevation: 1,218 meters (m)

Northing: 4,097,495.64 m (UTM Zone: 11 )

Latitude: 3701.2931

Easting: $585,362.42 \mathrm{~m}$ (UTM Zane: 1 )

Longitude: 11602.4748

Coordinate/Elevation Data Obtained from: North American Datum, 1927.

Site Access Route: Proceed on Mercury Highway north to Angle Road. Turn right (east) onto Angle Road and proceed approximately 0.8 mile. The epoxy test hole is approximately 300 feet south of the road.

\begin{tabular}{|c|c|}
\hline Waste Item(s) Originally at Síte & \multicolumn{1}{|c|}{ Apparent Waste Type* } \\
\hline Epoxy tar, trash can lid, and empty paint can & Ordinary \\
\hline
\end{tabular}

* Ordinary, Scray Metai, Ashestos, PCB, Salvageable, Hazardous, Radioactive, Mixed, Unknown, Other

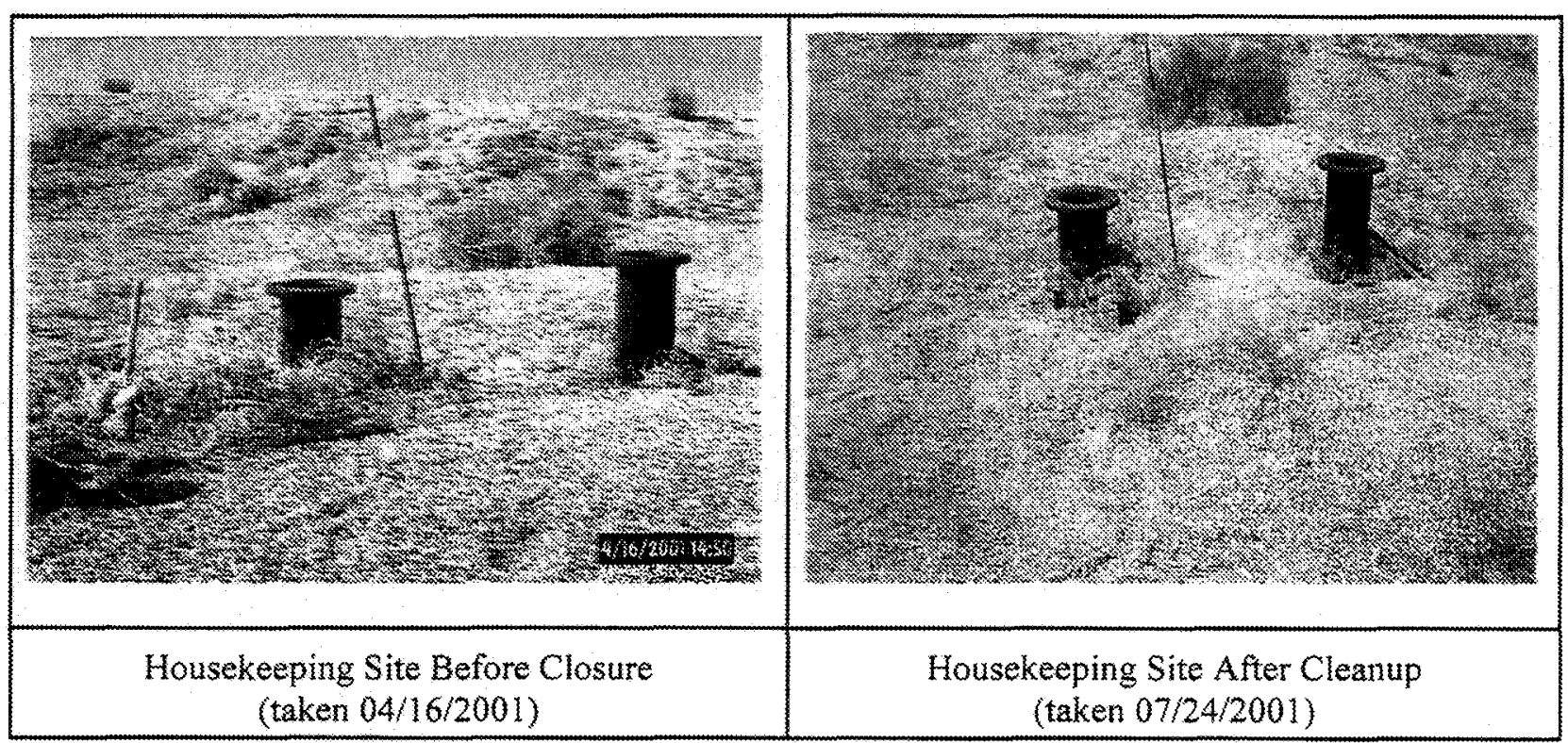

Current Site Description/Observations: This Corrective Action Site (CAS) was first identified by an International Technology Corporation (IT) field crew in a site survey performed on October 5, 1993. The epoxy tar and debris were removed on July 24,2001 , using shovels and a frontend loader and transported to the Area 6 Hydrocarbon Landfill. The site is currently clear of all waste material.

\section{$\mathrm{X}$ No Further Action Required at Housekeeping Site}

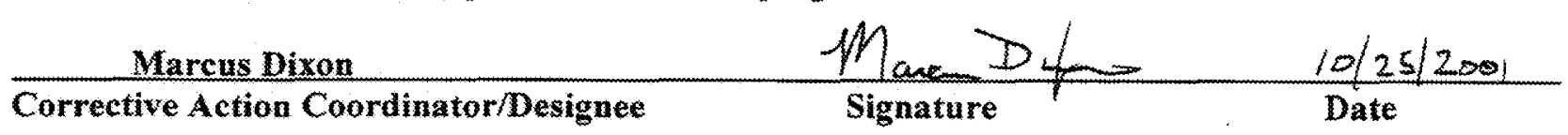




\section{Sectored Housekeeping Site Closure Verification Form}

Closure Verification Date: 07/25/2001

C.S Number (if applicable): 03-99-06

CAU Number (if applicable): 387

Sector Designation: NTS

Housekeeping Site General Location: Nevada Test Site, U-3kj crater

Elevation: 1,231 meters (m)

Northing: 4,097,423.25 m (UTM Zone: 11)

Latitude: 3701.2502

Easting: $586,048.16 \mathrm{~m}$ (UTM Zone: 11 )

Longitude: 11602.0128

Coordinate/Elevation Data Obtained from: North American Datum, 1927.

Site Access Route: Proceed on Mercury Highway noth to Angle Road. Tum right (northeast) on Angle Road and proceed to $3-08$ Road. Tum right (east) on 3-08 Road and proceed for approximately 0.65 mile to a skid trail that turns to the north (left). Proceed on the trail approximately 0.1 mile north to the U-3kj crater; go around to the northwest side of the crater.

\begin{tabular}{|l|l|}
\hline \multicolumn{1}{|c|}{ Waste Item(s) Orginally at Site } & \multicolumn{1}{|c|}{ Apparent Waste Type* } \\
\hline Epoxy tar & Ordinary \\
\hline * Ordinary, Scrap Metai, Astestos, $P C$ C Salyageazle Hazardous, Radiog
\end{tabular}

* Ordinary, Serap Metal, Asbestos, PCS, Salvageable, Hazatdous, Radionctive, Mfixed, Unknown, Other

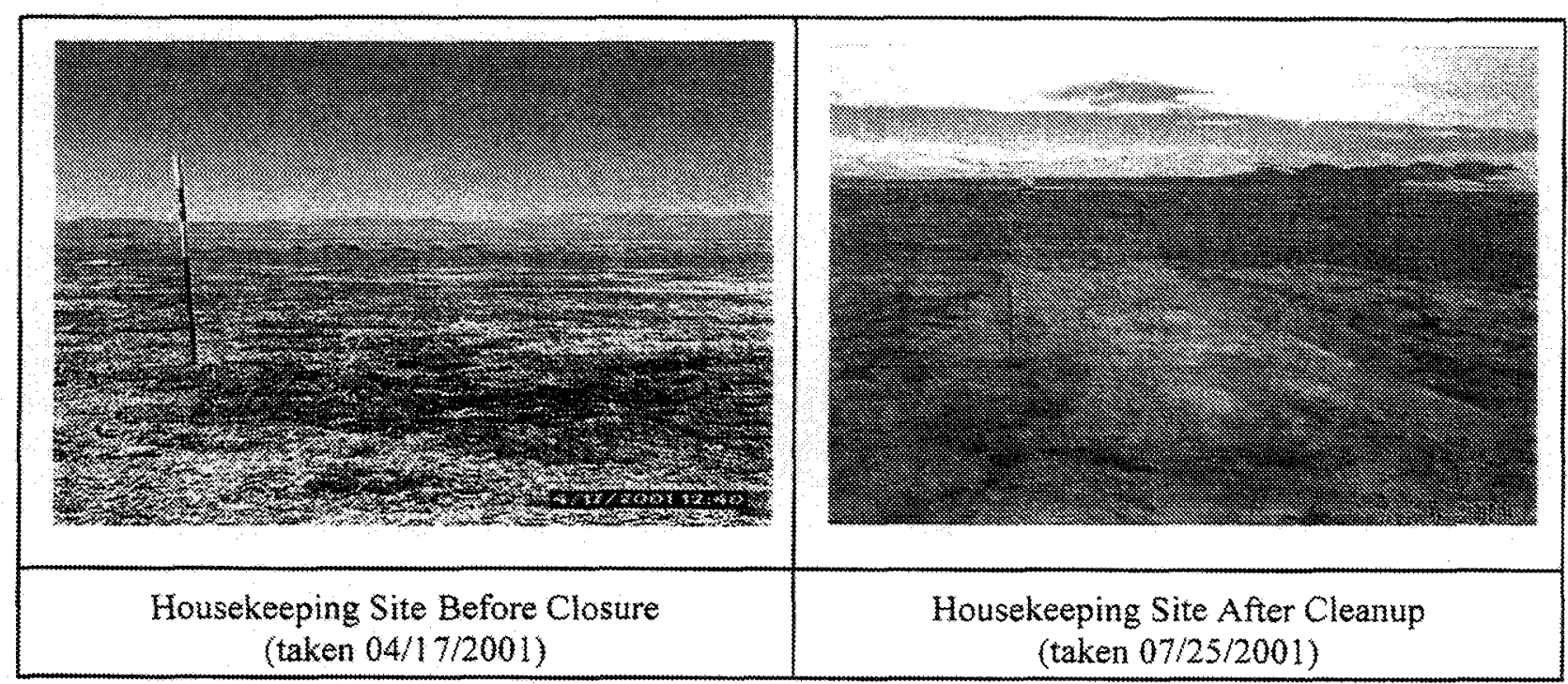

Current Site Description/Observations: This Corrective Action Site (CAS) was first identified by an International Technology Corporation (T) field crew in a site survey performed on July 15, 1999. The epoxy tar was removed on July 25, 2001, using a front-end loader and transported to the Area 6 Hydrocarbon Landfill. The site is currently clear of all waste material.

\section{$\mathrm{X}$ No Further Action Required at Housekeeping Site}

$\begin{array}{cccc}\text { Marcus Dixon } & M a n & D L & 10 / 25 / 2001 \\ \text { Corrective Action Coordinator/Designee } & \text { Signature } & \text { Date }\end{array}$




\section{Sectored Housekeeping Site Closure Verification Form}

Closure Verification Date: 08/17/2001

CAS Number (if applicable): 03-99-08

CAU Number (if applicable): 387

Sector Designation: NTS

Housekeeping Site General Location: Nevada Test Site, U-3gi crater

Elevation: 1,143 meters $(\mathrm{m})$

Northing: 4,098,511.03 m (UTM Zone: 11) Latitude: 3701.8330

Easting: $587,038.55 \mathrm{~m}$ (UTM Zone: 11$)$

Longitude: 11601.3373

Coordinate/Elevation Data Obtained from: North American Datum, 1927.

Site Access Route: Proceed on Mercury Highway north to Angle Road. Turn right (northeast) onto Angle Road and proceed to 3-07 Road (3B Road). Turn right (east) onto 3-07 Road and proceed to 3-12 Road. Proceed straight across the intersection to dirt road for approximately 0.25 mile. Turn left (north) at skid trail. Proceed 0.2 mile between two craters; site will be found straight ahead.

\begin{tabular}{|l|l|}
\hline \multicolumn{1}{|c|}{ Waste Item(s) Originally at Site } & \multicolumn{1}{c|}{ Apparent Waste Type* } \\
\hline Tar & Ordinary \\
\hline
\end{tabular}

* Ordinary, Scrap Metai, Asbestos, PCB, Salvageable, Kazardous, Radicactive, Mixed, Unhnown, Other

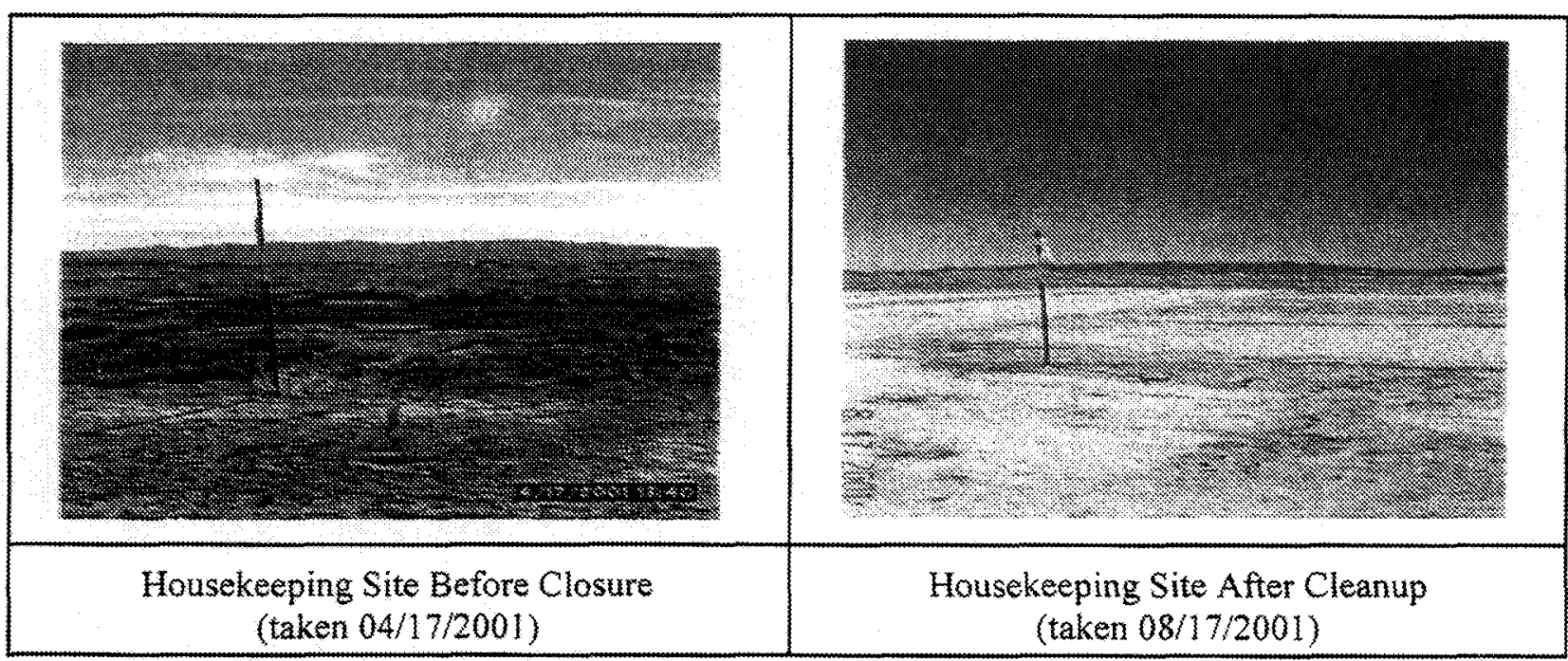

Current Site Description/Observations: This Corrective Action Site (CAS) was first identified by an International Technology Corporation (IT) field crew in a site survey performed on October 6, 2001. The tar spill was removed on August 17,2001, using shovels and a frontwend loader. The material was placed into B-25 containers due to its potential to be low-level waste (LLW). The site is currently clear of all waste material.

$\mathrm{X}$ No Further Action Required at Housekeeping Site

Marcus Dixon

Corrective Action Coordinator/Designee

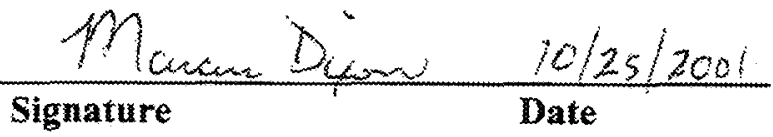

Signature

B-11 


\section{Sectored Housekeeping Site Closure Verification Form}

Closure Verification Date: 08/20/2001

CAS Number (if applicable): 03-99-17

CAU Number (if applicable) 387

Sector Designation: NTS

Housekeeping Site General Location: Nevada Test Stte, U-3cq crater

Elevation: 1,289 meters $(\mathrm{m})$

Northing: $4,100,978.75 \mathrm{~m}$ (UTM Zone: 11$)$

Latitude: 3703.1633

Easting: $587,795.88 \mathrm{~m}$ (UTM Zone: 11)

Longitude: 11600.8092

Coordinate/Elevation Data Obtained from: North American Datum, 1927.

Site Access Route: Proceed north on Mercury Highway to 3.03 Road and turn right (east). Proceed on 3.03 Road to 3-05 Road. Tum left (north) on 3.05 Road and proceed 0.6 mile to "Bilby Crater U-3cn" sign. Turn right (east) onto a dirt road for 0.2 mile to another dirt road and turn right. Continue until there is a " $Y$ " in the road and proceed straight ahead. Continue 0.7 mile along a skid trail and stay straight towards the U $3 \mathrm{cq}$ crater.

\begin{tabular}{|l|l|}
\hline Waste Item(s) Originally at Site & \multicolumn{1}{|c|}{ Apparent Waste Type* } \\
\hline Metal and amber glass bottle & Ordinary \\
\hline
\end{tabular}

* Ordinary, Scrap Metal, Asbestos, PCB, Salvageable, Hazardoux, Radicactive, Mixed, Uniknown, Other

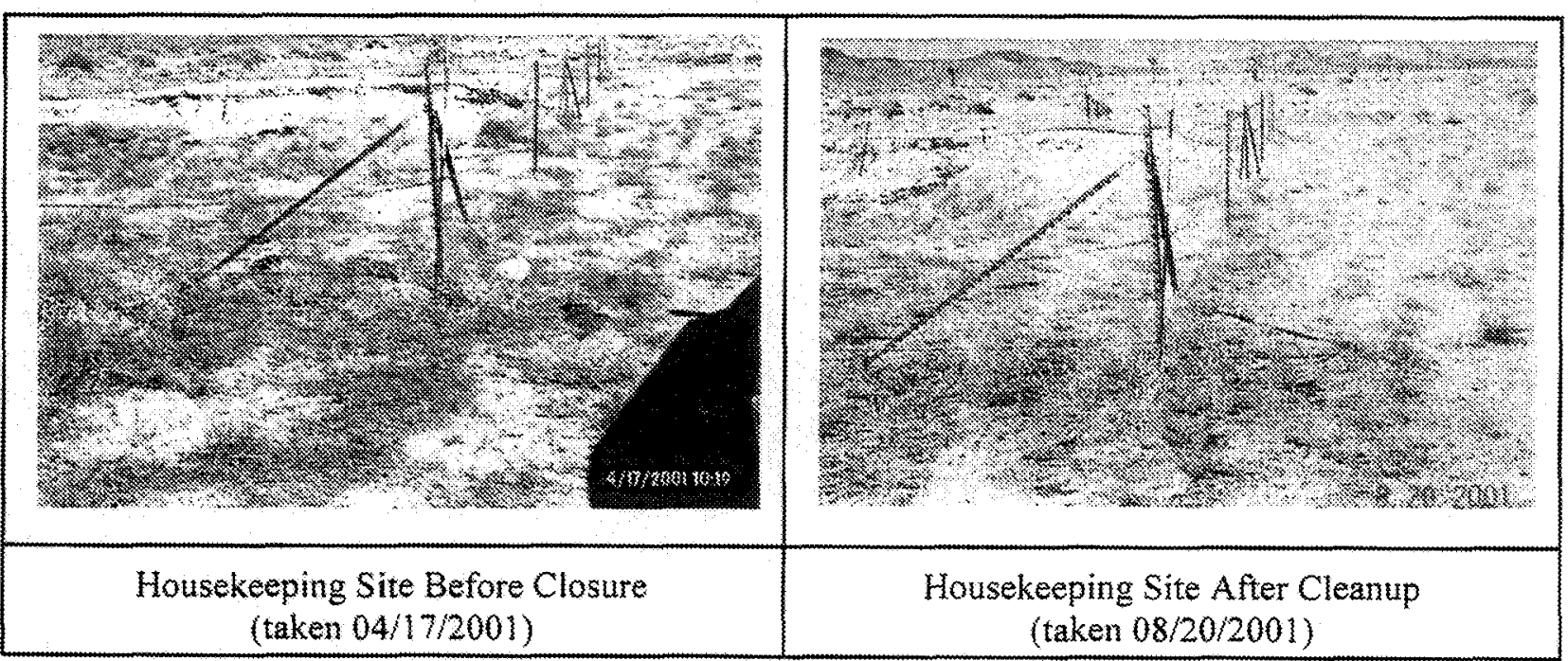

Current Site Description/Observations: This Corrective Action Site (CAS) was first identified by an International Corporation (T) field crew in a site survey performed on October 6,1993 . The amber glass bottle and metal were removed on August 20,2001. The liquid inside the bottle was sent to a lab; no constituents of concern were present. The site is currently clear of all waste material.

\section{$\mathrm{X}$ No Further Action Required at Housekeeping Site}

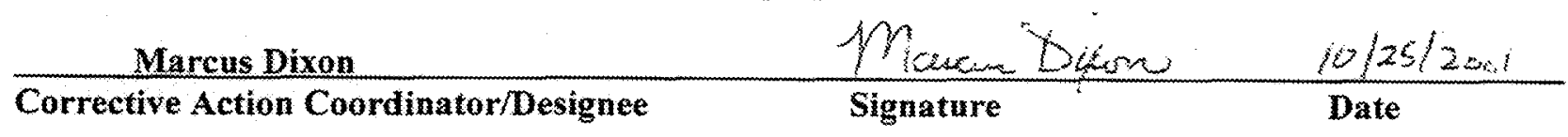




\section{Sectored Housekeeping Site Closure Verification Form}

Closure Verification Date: 08/17/2001

CAS Number (if applicable): 03-99-18

CAU Number (if applicable): 387

Sector Designation: NTS

Housekeeping Site General Location: Nevada Test Site, U-3as crater

Elevation: 1,243 meters ( $m$ )

Northing: $4,100,271.38 \mathrm{~m}$ (UTM Zone: 11)

Latitude: 3702.7928

Easting: 585,622.43 m (UTM Zone: 11 )

Longitude: 11602.2805

Coordinate/Elevation Data Obtained from: North American Datum, 1927.

Site Access Route: Proceed on Mercury Highway north to 3-03 Road. Turn right (east) on 3-03 Road and proceed approximately 0.8 mile to a dirt road on the south side of road. Tum south on the dirt road; $U$-3as crater will be on the east side of the road.

\begin{tabular}{|l|l|}
\hline \multicolumn{1}{|c|}{ Waste Item(s) Originally at Site } & \multicolumn{1}{|c|}{ Apparent Waste Type } \\
\hline Cement/ concrete & Industrial solid waste \\
\hline "Ordinary, Scrsp Metal, Asbestos, $P C B$, Salvageable, Hazardous, Radioative, Mixed, Unksown, Other
\end{tabular}

Ordinary, Serap Mekal, Asbestos, PCB, Salvageable, Hazadous, Radioantive, Mixed, Unknown, Other

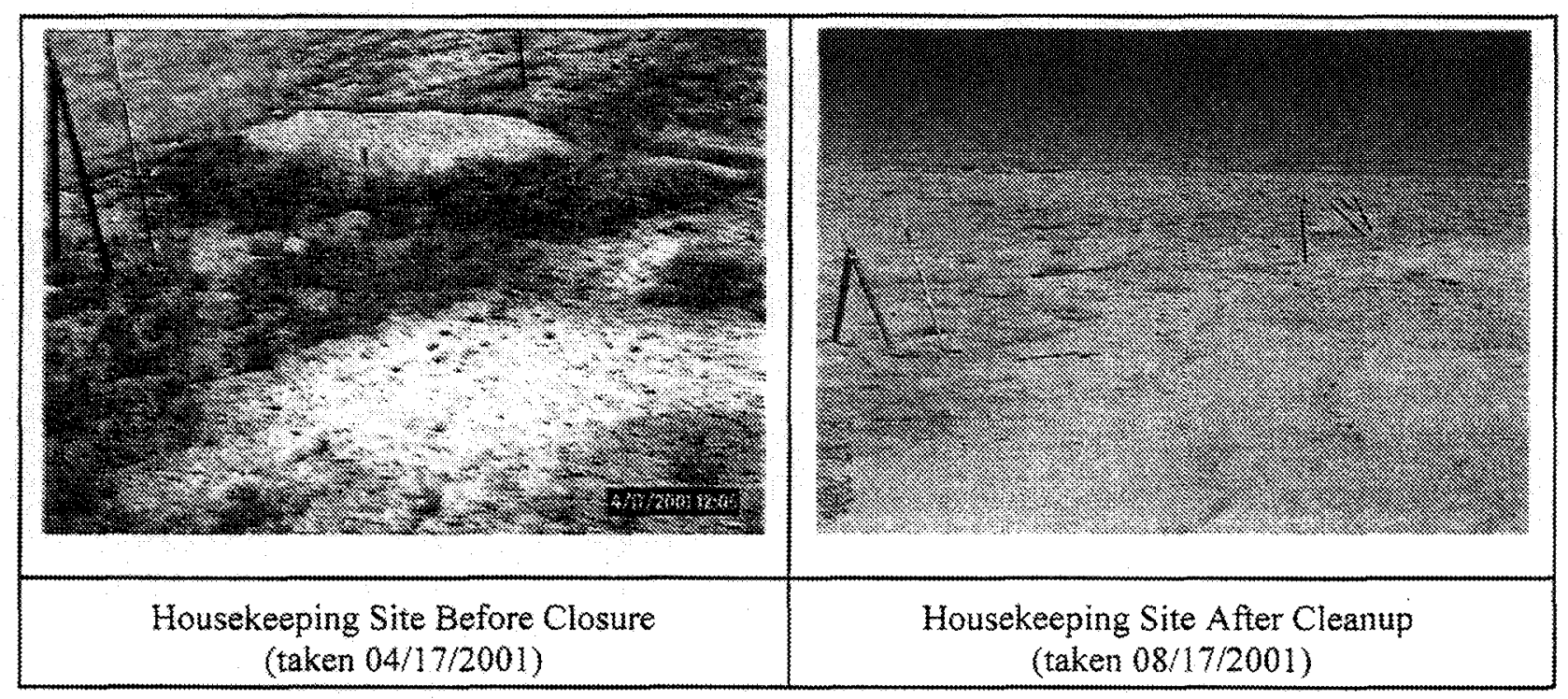

Current Site Description/Observations: This Corrective Action Site (CAS) was first identified by an Intemational Technology Corporation (T) field crew in a site survey performed on February 14, 1995. The cement/concrete spill was removed on August 17,2001, using a back hoe and transported to the Area 9 U10c Landfill. The site is currently clear of all waste material.

\section{$\mathrm{X}$ No Further Action Required at Housekeeping Site}

\begin{tabular}{|c|c|c|}
\hline Marcus Dixon & $\sin a$ & $200 i$ \\
\hline Corrective Action Coordinator $/$ Designee & Signature & Date \\
\hline
\end{tabular}




\section{Sectored Housekeeping Site Closure Verification Form}

Closure Verification Date: 08/21/2001

CAS Number (if applicable): 04-44-01

CAU Number (if applicable): 387

Sector Designation: NTS

Housekeeping Site General Location: Nevada Test Site, U-4as cellar Elevation: 1,354 meters ( $\mathrm{m}$ )

Northing: 3,996,463.24 m (UTM Zone: 11) Latitude: 3606.6825

Easting: $579,162.99 \mathrm{~m}$ (UTM Zone: 11 ) Longitude: 11607.2786

Coordinate/Elevation Data Obtained from: North American Datum, 1927.

Site Access Route: Proceed on Mercury Highway north, turn left (northwest) on Rainier Mesa Road. Tum left (west) on 404 Road and follow to Orange Road. Turn right (north) on Orange Road and proceed 1.25 miles (between RAD Safe Marker [RSM] 0.49 and 0-50). Turn left (southwest) and proceed 0.1 mile to the U-4as cellar. CAS $04-4403$ is due west of the U-4as sign.

\begin{tabular}{|c|c|}
\hline Waste Item(s) Originally at Site & \multicolumn{1}{|c|}{ Apparent Waste Type* } \\
\hline Cementgrout, wire, rope, and wood & Industrial solid waste and ordinary \\
\hline
\end{tabular}

* Ordinary Scrap Metal, Asbestos, PCB, Salvageable, Hazardous, Radioactive, Mixed, Unknown, Other

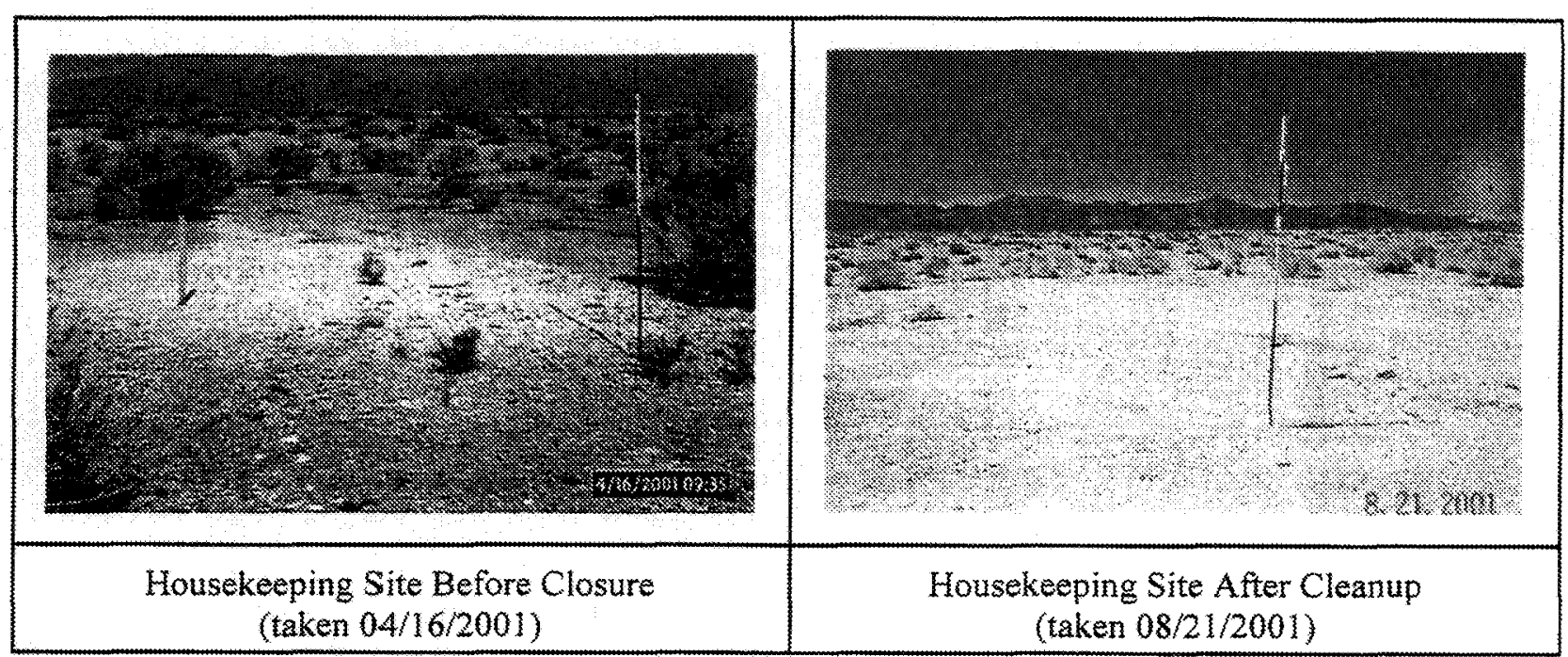

Current Site Description/Observations: This Corrective Action Site (CAS) was first identified by an International Technology Corporation (IT) field crew in a site survey performed on August 31, 1993. The cement/grout spill and debris were removed on August 21,2001, using a front-end loader and transported the Area 9 U100 Landfill. The site is currently clear of all waste material.

$\mathrm{X}$ No Further Action Required at Housekeeping Site

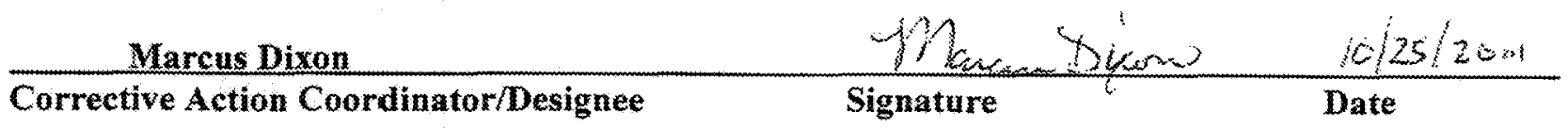




\section{Sectored Housekeeping Site Closure Verification Form}

Closure Verification Date: 08/21/2001

CAS Number (if applicable): 04-44-03

CAU Number (if applicable): 387

Sector Designation: NTS

Housekeeping Site General Location: Nevada Test Site, Uwat crater

Elevation: 1,264 meters (m)

Northing: 4,107,086.74 m (UTM Zone: 11) Latitude: 3706.5179

Easting: $578,105.54 \mathrm{~m}$ (UTM Zone: 11)

Longitude: 11607.3095

\section{Coordinate/Elevation Data Obtained from: North American Datum, 1927.}

Site Access Route: Take Mercury Highway north, turn left (northwest) on the Rainier Mesa Road. Turn left (west) on the $4-04$ Road and continue to the "T" intersection at the Orange Road. Tum right (north) and proceed 0.85 mile to the U-4at crater. The site is located on the northwest comer of Crater U-4at.

\begin{tabular}{|c|l|}
\hline Waste Item(s) Originally at Site & \multicolumn{1}{|c|}{ Apparent Waste Type* } \\
\hline Cement/drilling material, rope, and wood & Industrial solid waste and ordinary \\
\hline
\end{tabular}

* Ordinury, Scrap Metal, Ashestos, PCB, Salyggeable, hazardous, Gaúchoctive, Mixed, Unknown, Other

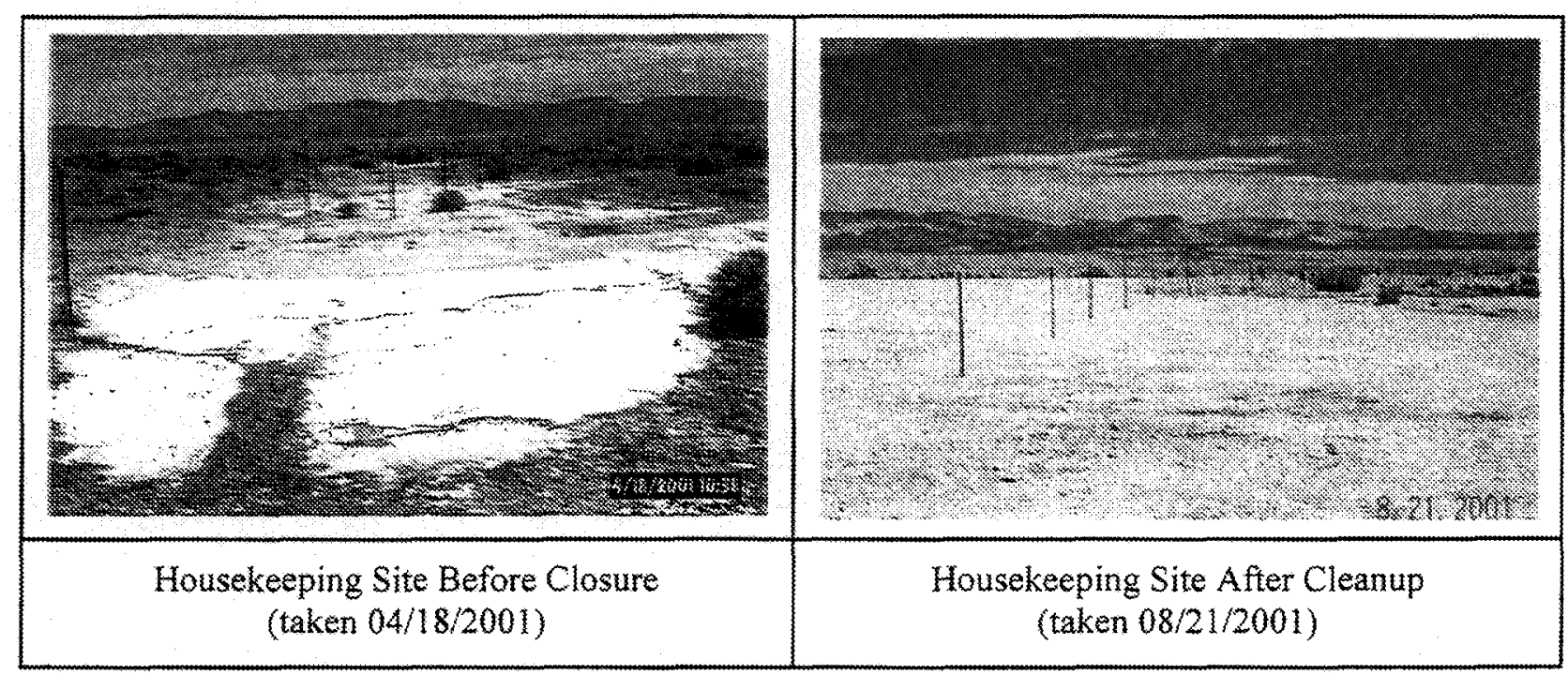

Current Site Description/Observations: This Corrective Action Site (CAS) was first identified by an Intemational Technology Corporation (T) field crew in a site survey performed on August 31, 1993. The drilling material was removed on August 21,2001, using a frontwend loader and transported to the Area 9 U10 L Landfill. The site is currently clear of all waste material.

\section{$\mathrm{X}$ No Further Action Required at Housekeeping Site}

\begin{tabular}{|c|c|c|}
\hline Marcus Dixon & $1 / \cos x+2 \cos 0$ & $10 / 25 / 200$ \\
\hline Corrective Action Coordinator/Designee & Signature & Date \\
\hline
\end{tabular}




\title{
Sectored Housekeeping Site Closure Verification Form
}

\author{
Closure Verification Date: $08 / 03 / 2001$ \\ CAS Number (if applicable): 07-99-02 (debris on cement pad) \\ CAU Number (if applicable): 387
}

Sector Designation: NTS

Housekeeping Site General Location: Nevada Test Site, UE-7ns crater Elevation: 1,350 meters (m)

Northing: 4,106,108.63 m (UTM Zone: 11)

Easting: $588,645.27 \mathrm{~m}$ (UTM Zone: 11 )

Latitude: 3705.9327

Longitude: 11600.1998

\section{Coordinate/Elevation Data Obtained from: North American Datum, 1927.}

Site Access Route: Proceed on Mercury Highway north to 303 Road. Turn right (east) on 3.03 Road and proceed to 3-05 Road. Turn left (north) onto $3-05$ Road, and at the fork in the road turn right (east). Proced 1.7 miles to RAD Safe Marker (RSM) 76.6. Tum right (east) at the dirt road (at a light blue fence post) and proceed 0.2 mile Tum left at another fork in the road. Follow for 0.3 mile to the site located behind a large white sign on the north side of the road.

\begin{tabular}{|l|l|}
\hline Waste Item(s) Originally at Site & \multicolumn{1}{|c|}{ Apparent Waste Type* } \\
\hline Wood, metal, wire, and rubber & Ordinary \\
\hline
\end{tabular}

* Ondinary, Scrap Netal Asbestos, PCB, Salvageable, Hazardous, Radtoactive, Mixed, Uniknown, Other

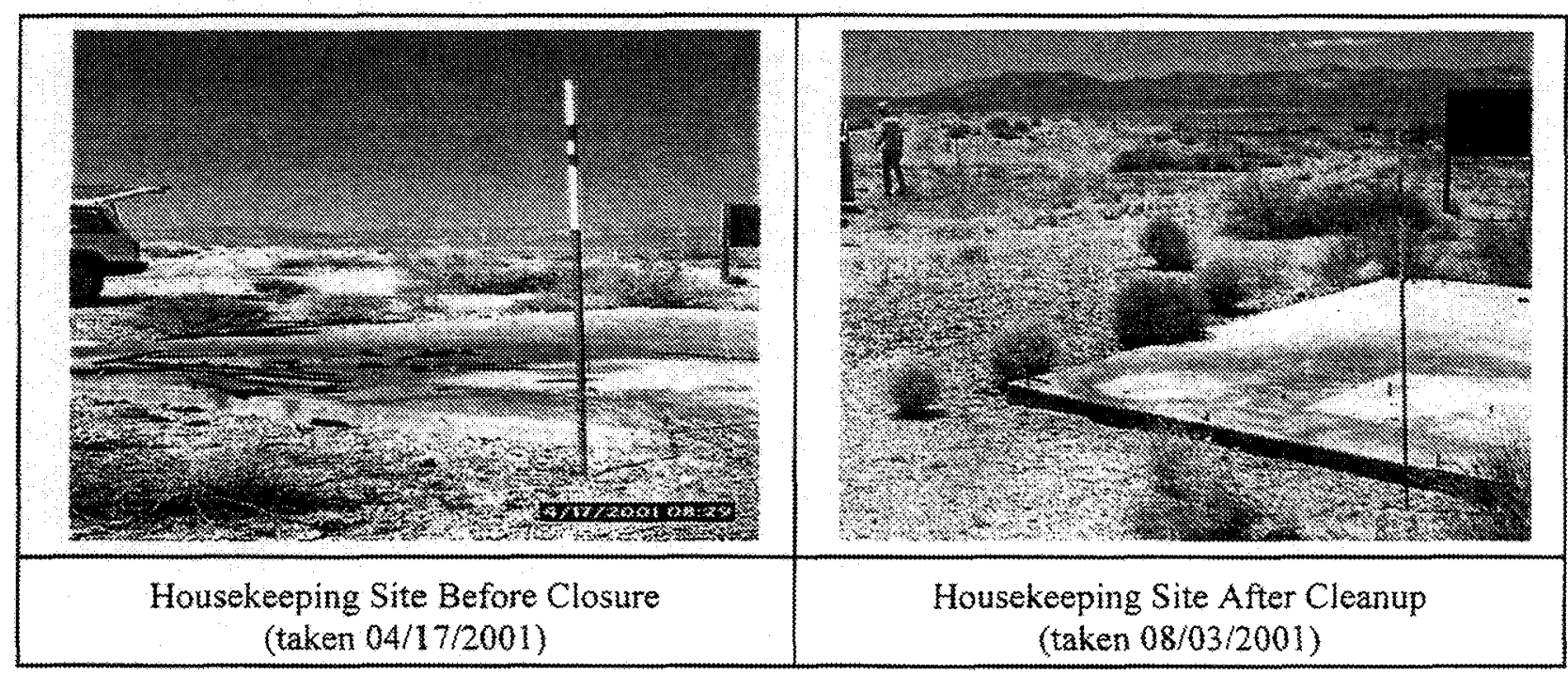

Current Site Description/Observations: This Corrective Action Site (CAS) was first identified by an International Technology Corporation (IT) field crew in a site survey performed September 9, 1993. The debris was removed on August 3,2001, using a front-end loader and shovels and transported to the Area 9 U10 Landfill. The site is currently clear of all waste material.

$\mathrm{X}$ No Further Action Required at Housekeeping Site

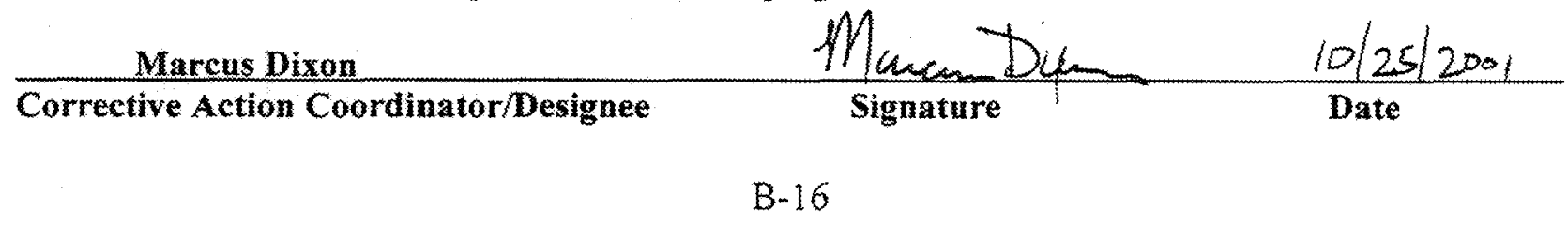




\section{Sectored Housekeeping Site Closure Verification Form}

Closure Verification Date: 08/03/2001

CAS Number (if applicable): 07-99.02 (pile of cement and debris)

CAU Number (if applicable): 387

Sector Designation: NTS

Housekeeping Site General Location: Nevada Test Site, UE-7ns crater

Elevation: 1,350 meters (m)

Northing: 4,106,108.63 m (UTM Zone: 11) Latitude: 3705.9327

Easting: $588,645.27 \mathrm{~m}$ (UTM Zone: 11)

Longitude: 11600.1998

Coordinate/Elevation Data Obtained from: North American Datum, 1927.

Site Access Route: Proceed on Mercury Highway north to 3-03 Road. Turn right (east) on 3-03 Road and proceed to 3-05 Road. Tum left (north) onto 3-05 Road and at the fork in the road tum right (east). Proceed 1.7 miles to RAD Safe Marker (RSM) 76.6. Turn right (east) at the dirt road (at a light blue fence post) and proceed 0.2 mile. Tum left at another fork in the road. Follow for 0.3 mile to the site located behind a large white sign on the north side of the road.

\begin{tabular}{|l|l|}
\hline Waste Item(s) Originally at Site & \multicolumn{1}{|c|}{ Apparent Waste Type* } \\
\hline Cement, wood, metal, and rubber & Industrial solid waste and ordinary \\
\hline
\end{tabular}

* Ordinary, Scrap Metal, Asbestos, PCB, Salvageable, Hazardous, Radionctive, Mixed, Unknown, Other

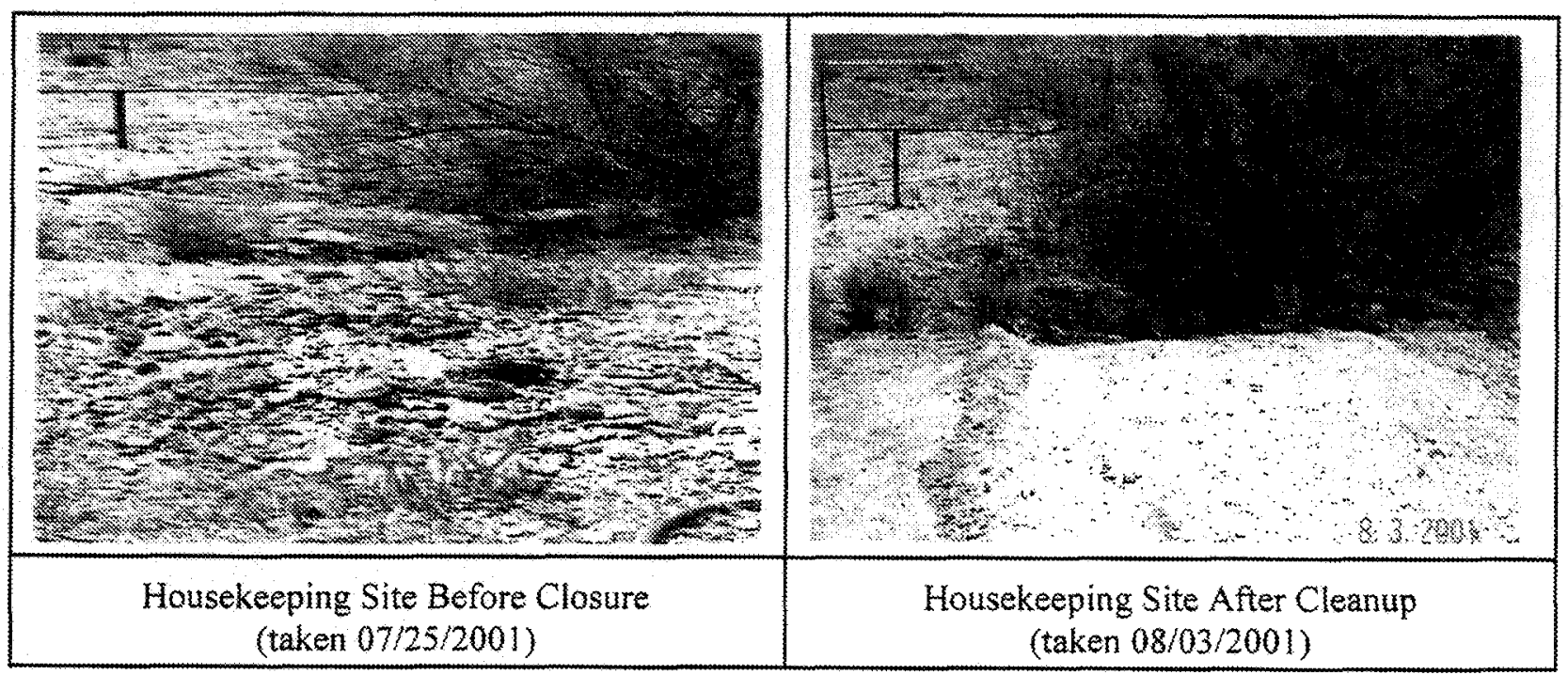

Current Site Description/Observations: This Corrective Action Site (CAS) was first identified by an International Technology Corporation (IT) field crew in a site survey performed September 9, 1993. The cement and debris was removed on August 3, 2001, using a front-end loader and shovels and transported. to the Area 9 U10c Landfill. The site is currently clear of all waste material.

\section{$x$ No Further Action Required at Housekeeping Site}

$\frac{\text { Marcus Dixon }}{\text { Corrective Action Coordinator/Designee }} \frac{M M_{\text {Signature }}}{10 / 25 / 200}$




\section{Sectored Housekeeping Site Closure Verification Form}

Closure Verification Date: $08 / 03 / 2001$

CAS Number (if applicable): 07-99-03 (epoxy tar spill)

CAU Number (if applicable): 387

Sector Designation: NTS

Housekeeping Site General Location: Nevada Test Site, U-7bg crater

Elevation: 1,335 meters $(\mathrm{m})$

Northing: $4,106,410.10 \mathrm{~m}$ (UTM Zone: 11 )

Latitude: 3706.0960

Easting: 588,597.82m (UTM Zone: 11)

Longitude: 11600.2297

Coordinate/Elevation Data Obtained from: North American Datum, 1927.

Site Access Route: Proceed on Mercury Highway north to 3-03 Road. Turn right (east) on 3-03 Road and proceed to 3-05 Road. Turn left (north) onto 3-05 Road and at the fork in the road tum right (east). Proceed 1.7 miles to RAD Safe Marker (RSM) 76.6. Tum right (east) at the dirt road (at a light biue fence post) and proceed 0.2 mile. Turn left at another fork in the road. Follow for 0.3 mile to CAS 07. 99-02. Continue straight past the concrete pad for approximately 200 feet to a wash. Go through the wash and on the other side make an immediate lef (north) and follow the dirt trail to the well yard. The site is located to the west of the well yard.

\begin{tabular}{|c|c|}
\hline Waste Item(s) Originally at Site & \multicolumn{1}{|c|}{ Apparent Waste Type* } \\
\hline Epoxy, wooden spool, and wire & Ordinary \\
\hline
\end{tabular}

* Otdinary, Scray Metal. Asbestos, PCB. Salyageable, Hazardous, Radicactive, Mixed, Unknown, Oher

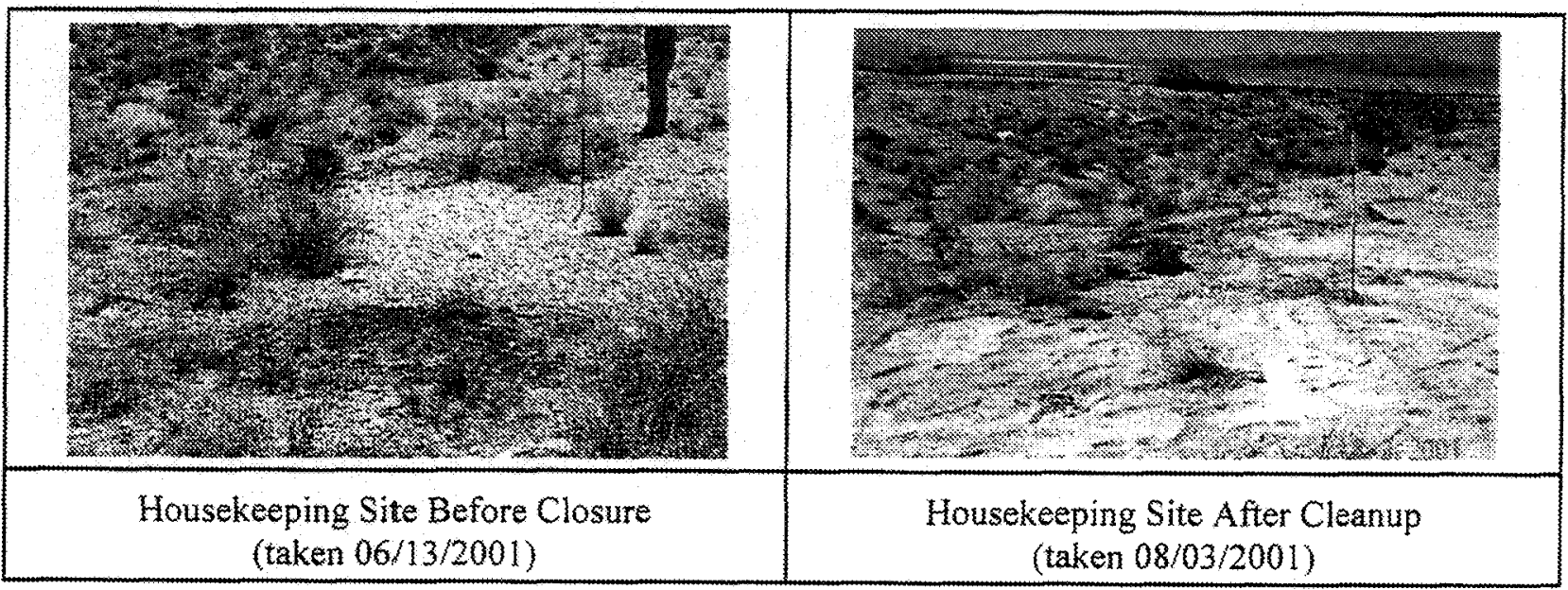

Current Site Description/Observations: This Corrective Action Site (CAS) was first identified by an International Technology Corporation (IT) field crew in a site survey performed on October 10, 1993. The two small epoxy spills and debris were removed on August 3,2001, using a front-end loader and transported to the Area $6 \mathrm{Hydrocarbon} \mathrm{Landfill.} \mathrm{The} \mathrm{site} \mathrm{is} \mathrm{currently} \mathrm{clear} \mathrm{of} \mathrm{all} \mathrm{waste} \mathrm{material.}$

$\mathrm{X}$ No Further Action Required at Housekeeping Site

\begin{tabular}{ccc} 
Marcus Dixon & Mlacen & 10/25/2001 \\
\hline Corrective Action Coordinator/Designee & Signature & Date
\end{tabular}




\section{Sectored Housekeeping Site Closure Verification Form}

Closure Verification Date: 09/25/2001

CAS Number (if applicable): $07-99-03$

CAU Number (if applicable): 387

Sector Designation: NTS

Housekeeping Site General Location: Nevada Test Site, U-7bg crater

Elevation: 1,336 meters (m)

Northing: 4,106,398.81 m (UTM Zone: 11)

Latitude: 3706.0898

Easting: $588,614.23 \mathrm{~m}$ (UTM Zone: 11 )

Longitude: 11600.2187

Coordinate/Elevation Data Obtained from: North American Datum, 1927.

Site Access Route: Proceed on Mercury Highway noth to 3-03 Road. Turn right (east) on 3-03 Road and proceed to 3-05 Road. Tum left (north) onto 3-05 Road and at the fork in the road turn right (east). Proceed 1,7 miles to RAD Safe Marker (RSM) 76.6. Turn right (east) at the dirt road (a t a light blue fence post) and proceed 0.2 mile. Tum left at another fork in the road. Follow for 0.3 mile to CAS 07. 99.02. Continue straight past the concrete pad for approximately 200 feet to a wash. Go through wash; on the other side make an inmediate left (north) and follow dirt trail to the well yard. The site is located to the west of the well yard.

\begin{tabular}{|l|l|}
\hline Waste Item(s) Originally at Site & \multicolumn{1}{|c|}{ Apparent Waste Type } \\
\hline Compressed gas cylinder (empty) & Ordinary \\
\hline
\end{tabular}

- Ordinary, Scrap Metal, Ashestos, PCE, Salvaguable, Kazardous, Radioactive, Mixed, Unknown, Other

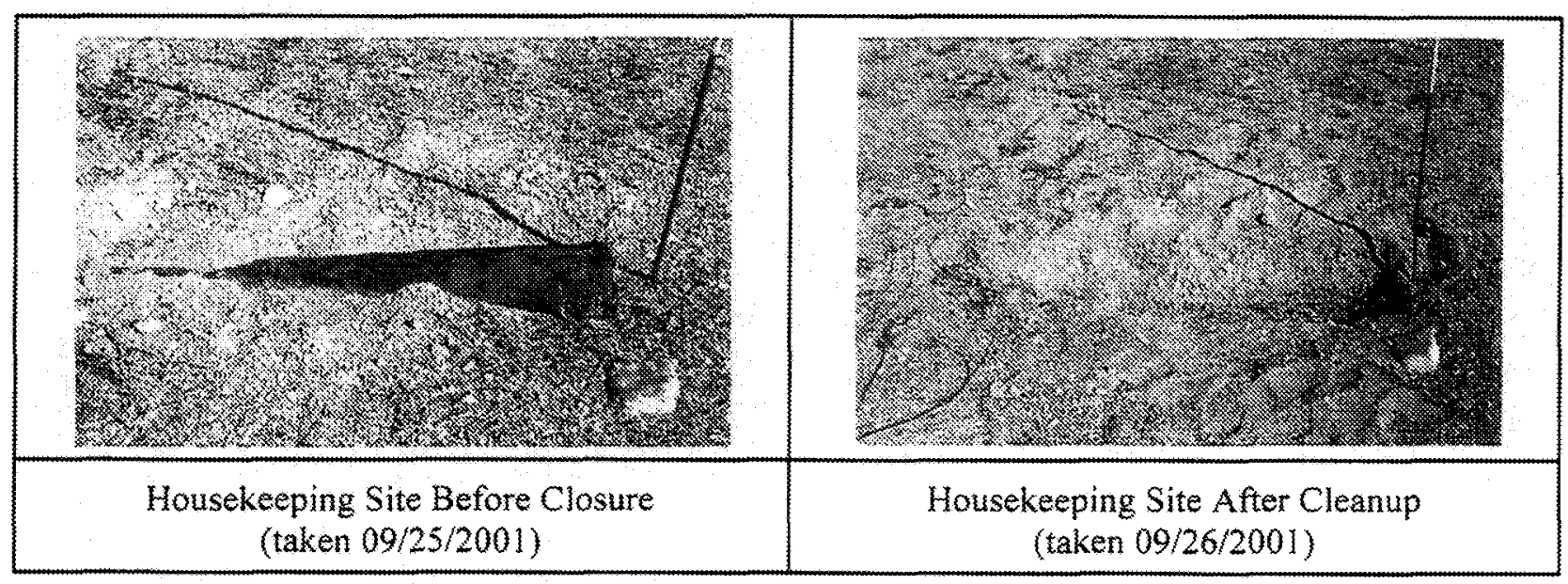

Current Site Description/Observations: This Corrective Action Site (CAS) was first identified by an International Technology Corporation (IT) field crew in a site survey performed on October 14, 1993. The gas cylmder was removed on September 25,2001, by charging with explosives to neutralize it and then transported to the Area 9 U10e Landfill. The site is currently clear of all waste material.

\section{$\underline{x}$ No Further Action Required at Housekeeping Site}

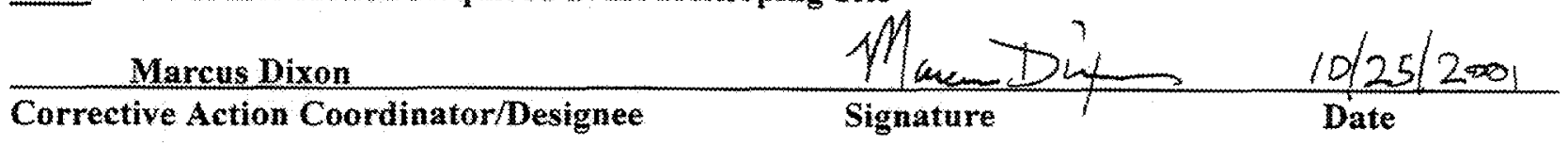




\section{DISTRIBUTION LIST}




\section{DISTRIBUTION LIST}

*Provide copy of initial distribution of Revision 0; remainder of list gets Revision 0 if approved without changes. The entire list receives Revision 1, if issued.

Nevada Department of Environmental Protection

Paul Liebendorfer

2 (Controlled)*

Bureau of Federal Facilities

Division of Environmental Protection

333 W. Nye Lane, Room 138

Carson City, NV 89706-0866

Supervisor, Las Vegas Office

1 (Controlled)*

Bureau of Federal Facilities

Division of Environmental Protection

555 E. Washington, Suite 4300

Las Vegas, NV 89010-1043

\section{U.S. Department of Energy}

Janet Appenzeller-Wing

1 (Uncontrolled)*

Environmental Restoration Division

U.S. Department of Energy

National Nuclear Security Administration

Nevada Operations Office

P.O. Box $98518, \mathrm{M} / \mathrm{S} 505$

Las Vegas, NV 89193-8518

Sabine Curtis

1 (Uncontrolled)*

Environmental Restoration Division

U.S. Department of Energy

National Nuclear Security Administration

Nevada Operations Office

P.O. Box 98518 , M/S 505

Las Vegas, NV 89193-8518

Sabrina Lawrence

1 (Controlled)*

Environmental Restoration Division

U.S. Department of Energy

National Nuclear Security Administration

Nevada Operations Office

P.O. Box 98518, M/S 505

Las Vegas, NV 89193-8518 


\section{DISTRIBUTION LIST (continued)}

\section{U.S. Department of Energy (continued)}

U.S. Department of Energy

National Nuclear Security Administration

Nevada Operations Office

Public Reading Facility

P.O. Box 98521, M/S NLV040

Las Vegas, NV 89193-8521

U.S. Department of Energy

National Nuclear Security Administration

Nevada Operations Office

Technical Information Resource Center

P.O. Box 98518, M/S 505

Las Vegas, NV 89193-8518

U.S. Department of Energy

Office of Scientific and Technical Information

P.O. Box 62

Oak Ridge, TN 37831-0062
1 (Controlled \&

1 Uncontrolled)

1 (Uncontrolled

\section{Bechtel Nevada}

Correspondence Control

1 (Uncontrolled \& 1 electronic copy)

Bechtel Nevada

P.O. Box 98521 , M/S NLV008

Las Vegas, NV 89193-8521

Environmental Management Library

1 (Uncontrolled)*

Bechtel Nevada

P.O. Box 98521, M/S NLV080

Las Vegas, NV 89193-8521

Marcus Dixon

1 (Uncontrolled)*

Bechtel Nevada

P.O. Box 98521, M/S NTS306

Las Vegas, NV 89193-8521

Ann Heidema

1 (Uncontrolled)

Bechtel Nevada

P.O. Box 98521 , M/S NLV022

Las Vegas, NV 89193-8521

1 (Uncontrolled)* 


\section{DISTRIBUTION LIST (continued)}

\section{Bechtel Nevada (continued)}

Thomas Fitzmaurice

1 (Uncontrolled)*

Bechtel Nevada

P.O. Box 98521 , M/S NTS306

Las Vegas, NV 89193-8521

Wayne Johnson

1 (Uncontrolled)*

Bechtel Nevada

P.O. Box 98521 , M/S NTS306

Las Vegas, NV 89193-8521

Steve Nacht

1 (Uncontrolled)*

Bechtel Nevada

P.O. Box 98521, M/S NTS306

Las Vegas, NV 89193-8521

\section{IT Corporation}

Lynn Kidman

1 (Uncontrolled)*

IT Corporation

P.O. Box 93838, M/S 439

Las Vegas, NV 89193-8521

Garry Romano

1 (Controlled)

IT FFACO Support Office

IT Corporation

P.O. Box 93838, M/S 439

Las Vegas, NV 89193-8521

\section{State of Nevada}

Manager, Northem Nevada

1 (Uncontrolled)

FFACO Public Reading Room

Nevada State Library and Archives Federal Publications

100 North Stewart Street

Carson City, NV 89701-4285 
CLOSURE REPORT - CAU 387

Spill Sites and Releases Section: Distribution Revision: 0

Date: October 2001

\section{THIS PAGE INTENTIONALLY LEFT BLANK}

